\title{
Competitive binding of STATs to receptor phospho-Tyr motifs accounts for altered cytokine responses in autoimmune disorders
}

Stephan Wilmes ${ }^{1^{*}}$, Polly-Anne Jeffrey ${ }^{2^{*}}$, Jonathan Martinez-Fabregas ${ }^{1}$, Maximillian Hafer ${ }^{3}$, Paul Fyfe ${ }^{1}$, Elizabeth Pohler ${ }^{1}$, Silvia Gaggero ${ }^{4}$, Martín López-García ${ }^{2}$, Grant Lythe ${ }^{2}$, Thomas Guerrier $^{5}$, David Launay ${ }^{5}$, Mitra Suman ${ }^{4}$, Jacob Piehler ${ }^{3}$, Carmen Molina-París ${ }^{2 \#}$ and Ignacio Moraga $^{1 \#}$

${ }^{1}$ Division of Cell Signalling and Immunology, School of Life Sciences, University of Dundee, Dundee, UK.

${ }^{2}$ Department of Applied Mathematics, School of Mathematics, University of Leeds, Leeds, UK.

${ }^{3}$ Department of Biology and Centre of Cellular Nanoanalytics, University of Osnabrück, Osnabrück, Germany.

${ }^{4}$ Université de Lille, INSERM UMR1277 CNRS UMR9020-CANTHER and Institut pour la Recherche sur le Cancer de Lille (IRCL), Lille, France.

${ }^{5}$ Univ. Lille, Inserm, CHU Lille, U1286 - INFINITE - Institute for Translational Research in Inflammation, F-59000 Lille, France.

* These authors contributed equally to this work

\# These authors share senior authorship

\section{ABSTRACT}

Cytokines elicit pleiotropic and non-redundant activities despite strong overlap in their usage of receptors, JAKs and STATs molecules. We use IL-6 and IL-27 to ask how two cytokines activating the same signaling pathway have different biological roles. We found that IL-27 induces more sustained STAT1 phosphorylation than IL-6, with the two cytokines inducing comparable levels of STAT3 phosphorylation. Mathematical and statistical modelling of IL-6 and IL-27 signaling identified STAT3 binding to GP130, and STAT1 binding to IL-27R $\alpha$, as the main dynamical processes contributing to sustained pSTAT1 by IL-27. Mutation of Tyr613 on IL-27R $\alpha$ decreased IL-27-induced STAT1 phosphorylation by $80 \%$ but had limited effect on STAT3 phosphorylation. Strong receptor/STAT coupling by IL-27 initiated a unique gene expression program, which required sustained STAT1 phosphorylation and IRF1 expression and was enriched in classical Interferon Stimulated Genes. Interestingly, the STAT/receptor coupling exhibited by IL-6/IL-27 was altered in patients with Systemic lupus erythematosus (SLE). IL-6/IL-27 induced a more potent STAT1 activation in SLE patients than in healthy controls, which correlated with higher STAT1 expression in these patients. Partial inhibition of JAK activation by sub-saturating doses of Tofacitinib specifically lowered the levels of STAT1 activation by IL-6. Our data show that receptor and STATs concentrations critically contribute to shape cytokine responses and generate functional pleiotropy in health and disease. 


\section{INTRODUCTION}

IL-27 and IL-6 both have intricate functions regulating inflammatory responses (1). IL-27 is a hetero-dimeric cytokine comprised of p28 and EBI3 subunits (2). IL-27 exerts its activities by binding GP130 and IL-27Ra receptor subunits in the surface of responsive cells, triggering the activation of the JAK1/STAT1/STAT3 signaling pathway. IL-27 elicits both pro- and antiinflammatory responses, although the later activity seems to be the dominant one (3). IL-27 stimulation inhibits ROR $\gamma$ t expression, thereby suppressing Th-17 commitment and limiting subsequent production of pro-inflammatory IL-17 $(4,5)$. Moreover, IL-27 induces a strong production of anti-inflammatory $\mathrm{IL}-10$ on (Tbet ${ }^{+}$and FoxP3-) Tr-1 cells (6-8) further contributing to limit the inflammatory response. IL- 6 engages a hexameric receptor complex comprised of each of two copies of IL-6R $\alpha$, GP130 and IL-6 (9), triggering the activation, as IL-27 does, of the JAK1/STAT1/STAT3 signaling pathway. However, opposite to IL-27, IL-6 is known as a paradigm pro-inflammatory cytokine $(10,11)$. IL-6 inhibits lineage differentiation to Treg cells $(12)$ while promoting Th-17 $(13,14)$, thus supporting its pro-inflammatory role. How IL-27 and IL-6 elicit opposite immuno-modulatory activities despite activating almost identical signaling pathways is currently not completely understood.

The relative and absolute STATs activation levels seem to have intricate roles, which lead to a strong signaling and functional plasticity by cytokines. Although IL-6 robustly activates STAT3, it is capable to mount a considerable STAT1 response as well (15). Moreover, in the absence of STAT3, IL-6 induces a strong STAT1 response comparable to IFN $\gamma$ - a prototypic STAT1 activating cytokine (16). Likewise, the absence of STAT1 potentiates the STAT3 response for IL-27, which normally elicits a strong STAT1 response, rendering it to mount an IL-6-like response (15). Furthermore, negative feedback mechanisms like SOCSs and phosphatases have been described as critical players influencing STAT1 and STAT3 phosphorylation kinetics and thereby shaping their signal integration for GP130-utilizing cytokines (17-20). Yet, how all these molecular components are integrated by a given cell to produce the desired response is still an open question. Among the IL-6/IL-12 cytokine family, IL-27 exhibits a unique STAT activation pattern. The majority of GP130-engaging cytokines activate preferentially STAT3, with activation of STAT1 being an accessory or balancing component $(21,22)$. IL-27, however, triggers STAT1 and STAT3 activation with high potency (23). Indeed, different studies have shown that IL-27 responses rely on either STAT1 (24-26) or STAT3 activation $(7,27)$. Moreover, recent transcriptomics studies showed that in the absence of STAT3, IL-6 and IL-27 lost more than $75 \%$ of target gene induction. Yet, STAT1 was the main factor driving the specificity of the IL-27 versus the IL- 6 response, highlighting a critical interplay of STAT1 and STAT3 engagement (28).

While the biological responses induced by IL-27 and IL-6 have been extensively studied (3, $11)$, the very initial steps of signal activation and kinetic integration by these two cytokines have not been comprehensively analysed. Since the different biological outcomes elicited by IL-27 and IL-6 are most likely encoded in the early events of cytokine stimulation, here we specifically aimed to identify the molecular determinants underlying functional selectivity by IL-27 in human T-cells. We asked how a defined cytokine stimulus is propagated in time over multiple layers of signaling to produce the desired response. To this end, we probed IL-27 and IL-6 signaling at different scales, ranging from cell surface receptor assembly and early STAT1/3 effector activation to an unbiased and quantitative multi-omics approach: phosphoproteomics after early cytokine stimulation, kinetics of transcriptomic changes and alteration of the T-cell proteome upon prolonged cytokine exposure. 
IL-6 and IL-27 induced similar levels of assembly of their respective receptor complexes, which resulted in comparable phosphorylation of STAT3 by the two cytokines. IL-27, on the other hand, triggered a more sustained STAT1 phosphorylation. To decipher the molecular events which determine sustained STAT1 phosphorylation by IL-27, we mathematically model the STAT1 and STAT3 signaling kinetics induced by each of these cytokines. We identified differential binding of STAT1 and STAT3 to IL-27R $\alpha$ and GP130, respectively, as the main factor contributing to a sustained STAT1 activation by IL-27. At the transcriptional level, IL-27 triggered the expression of a unique gene program, which strictly required the cooperative action between sustained pSTAT1 and IRF1 expression to drive the induction of an interferonlike gene signature that profoundly shaped the T-cell proteome. Interestingly, our mathematical models of IL- 6 and IL-27 signaling predicted that changes in receptor and STAT expression could fundamentally change the magnitude and timescale of the IL-6 and IL-27 responses. We found high levels of STAT1 expression in SLE patients when compared to healthy donors, which correlated with biased STAT1 responses induced by IL-6 and IL-27 in these patients. Strikingly, we could specifically inhibit STAT1 activation by IL-6 using suboptimal doses of the JAK inhibitor Tofacitinib. This could provide a new strategy to specifically target individual STATs engaged by cytokines. 


\section{RESULTS:}

\section{IL-27 induces a more sustained STAT1 activation than HyplL-6 in human Th-1 cells}

IL-6 and IL-27 are critical immuno-modulatory cytokines. While IL-6 engages a hexameric surface receptor comprised of two molecules of IL-6R $\alpha$ and two molecules of GP130 to trigger the activation of STAT1 and STAT3 transcription factors (Figure 1a), IL-27 binds GP130 and IL-27R $\alpha$ to trigger activation of the same STATs molecules (Figure 1a). Despite sharing a common receptor subunit, GP130, and activating similar signaling pathways, these two cytokines exhibit non-redundant immuno-modulatory activities, with IL-6 eliciting a potent proinflammatory response and IL-27 acting more as an anti-inflammatory cytokine. Here, we set to investigate the molecular rules that determine the functional specificity elicited by IL-6 and IL-27 using human Th-1 cells as a model experimental system. Due to the challenging recombinant expression of the human IL-27, we have recombinantly produced a murine single-chain variant of IL-27 (p28 and EBI3) which cross-reacts with the human receptors and triggers potent signaling, comparable to the signaling output produced by commercial human IL-27 (29) (Supp. Fig. 1a). In addition, we have used a linker-connected single-chain fusion protein of IL-6R $\alpha$ and IL-6 termed HyperIL-6 (HyplL-6) (30) to diminish IL-6 signaling variability due to changes in IL-6R $\alpha$ expression during $T$ cell activation (31).

CD4+ T cells from human buffy coat samples were isolated by magnetic activated cell sorting (MACS) and grew under Th-1 polarizing conditions. Th-1 cells were then used to study in vitro signaling by IL-27 and IL-6 (Supp. Fig. 1b). We took advantage of a barcoding methodology allowing high-throughput multiparameter flow cytometry to perform detailed dose/response and kinetics studies induced by HyplL-6 and IL-27 in Th-1 cells (32) (Supp. Fig. 1b). Doseresponse experiments with IL-27 and HyplL-6 on Th-1 cells showed concentration-dependent phosphorylation of STAT1 and STAT3. Phosphorylation of STAT1/3 was more sensitive to activation by IL-27 with an $\mathrm{EC}_{50}$ of $\sim 20 \mathrm{pM}$ compared to $\sim 400 \mathrm{pM}$ for HyplL-6 (Figure 1b). Despite this difference in sensitivity, both cytokines yielded the same activation amplitude for pSTAT3. For PSTAT1, however, we observed a significantly reduced maximal amplitude for HyplL-6 relative to IL-27 (Figure 1b). We next performed kinetic studies to assess whether the poor STAT1 activation by HyplL-6 was a result from different activation kinetics. For STAT3, we saw the peak of phosphorylation after $\sim 15-30$ minutes, followed by a gradual decline. Both cytokines exhibited an almost identical sustained pSTAT3 profile, with $\sim 20 \%$ of activation still seen after $3 \mathrm{~h}$ of continuous stimulation. Interestingly, IL-27 did not only activate STAT1 with higher amplitude but also more sustained than HyplL-6 (Figure 1c). This could be better appreciated when pSTAT1 levels were normalized to maximal MFI for each cytokine, with IL27 inducing clearly a more sustain phosphorylation of STAT1 than HyplL-6 (Supp. Fig. 1c). The same phenotype was observed in other T-cell subsets of activated PBMCs (Supp. Fig. 1d). As cell surface GP130 levels are significantly reduced upon T-cell activation (33), we next investigated whether the transient STAT1 activation profile induced by HyplL-6 resulted from limited availability of GP130. For that we generated a RPE1 cell clone stably expressing ten times higher levels of GP130 in its surface (Figure 1d, right panel). Stimulation of this RPE1 clone with HyplL-6 resulted in a more sustained activation of STAT3, with very little effect on STAT1 activation kinetics when compared to RPE1 wild type cells, suggesting that GP130 receptor density does not contribute to the transient STAT1 activation kinetics elicited by HyplL-6 (Figure 1d).

\section{Ligand-induced cell-surface receptor assembly by IL-27 and HypIL-6}


We next investigated whether IL-27 and HyplL-6 elicited differential cell surface receptor engagement that could explain their distinct signaling output. For that, we measured the dynamics of receptor assembly in the plasma membrane of live cells by simultaneous dualcolour total internal reflection fluorescence (TIRF) imaging. RPE1 cells were chosen as a model experimental system since they do not express endogenous IL-27R $\alpha$ (Supp. Fig. 1e). We used previously described RPE1 GP130 KO cells (Supp. Fig. 2a) (34) to transfect and express tagged variants of IL-27R $\alpha$ and GP130, to allow quantitative site-specific fluorescence cell surface labelling by dye-conjugated nanobodies (NBs) (Figure 1e) as recently described in (35). For both IL-27R $\alpha$ and GP130 we found a random distribution and unhindered lateral diffusion of individual receptor monomers (Figure 1f). Single molecule colocalization combined with co-tracking analysis was then used to identify correlated motion of IL-27R $\alpha$ and GP130 which was taken as a readout for receptor heterodimer formation (36) (Figure 1f, Figure 1 supp. Movie 1). In the resting state, we did not observe pre-assembly of IL-27R $\alpha$ and GP130. However, after stimulation with IL-27 we found substantial heterodimerization (Figure $1 \mathrm{f} \& 1 \mathrm{~g}$, Supp. Fig. 2b, Figure 1 supp. Movie $1 \& 2$ ). At elevated laser intensities, bleaching analysis of individual complexes confirmed a one-to-one (1:1) complex stoichiometry of IL-27R $\alpha$ and GP130, whereas single-molecule Förster resonance energy transfer (FRET) further corroborated close molecular proximity of the two receptor chains (Figure 1h). We also observed association and dissociation events of receptor heterodimers, pointing to a dynamic equilibrium between monomers and dimers as proposed for other heterodimeric cytokine receptor systems (37, 38) (Figure1 supp. Movie 3).

To measure homodimerization of GP130 by HyplL-6, we stochastically labelled GP130 with equal concentrations of the same NB species conjugated to either of the two dyes (39). We saw strong homodimerization of GP130 after stimulation with HyplL-6 (Figure 1g, Supp. Fig. 2b , Figure 1 supp. Movie 4). Homodimerization was confirmed either by singlecolor dual-step bleaching or dual-color single-step bleaching as shown for other homodimeric cytokine receptors (Supp. Fig. 2c) (40). For both cytokine receptor systems, we saw a cytokine-induced reduction of the diffusion mobility, which has been ascribed to increased friction of receptor dimers diffusing in the plasma membrane. However, we note that HyplL-6 stimulation impaired diffusion of GP130 more strongly than IL-27 did, possibly indicating faster receptor internalization (Supp. Fig. 2d). Based on the dimerization data, we were able to calculate the two-dimensional equilibrium dissociation constants $\left(K_{D}^{2 D}\right)$ according to the law of mass action for a dynamic monomer-dimer equilibrium: for IL-27-induced heterodimerization of IL-27R $\alpha$ and GP130, we calculated a 2D K $\mathrm{K}_{\mathrm{D}}$ of $\sim 0.81 \mu \mathrm{m}^{-2}$. In activated T-cells with high levels and a significant excess of IL-27R $\alpha$ over GP130, this $K_{D}^{2 D}$ ensures strong receptor assembly by IL-27 (41). The 2D K for GP130 homodimerization by HyplL-6 was $\sim 0.21 \mu \mathrm{m}^{-2}$. This higher affinity is most likely due to the two high-affinity binding sites engaged in the hexameric receptor complex (9). However, in T-cells the expression of GP130 can be particularly low, thus, probably limiting HyplL-6. Taken together, these experiments marked ligand-induced receptor assembly as the initial step triggering downstream signaling for both IL-27 and HyplL-6, with no obvious differences in their receptor activation mechanism which could support the observed more sustained STAT1 activation elicited by IL-27.

\section{Mathematical and statistical analysis of HyplL-6 and IL-27 induced STAT kinetic responses}

To gain further insight into the molecular rules and kinetics that define IL-27 sustained STAT1 phosphorylation, we developed two mathematical models of the initial steps of HyplL-6 and 
IL-27 receptor-mediated signaling, respectively. The mathematical model for each cytokine considers the following events: i) cytokine association and dissociation to a receptor chain (Figure 2a, Supp. Fig. 3a and 3b, top panel), ii) cytokine-induced dimer association and dissociation (Supp. Fig. 3a and 3b, bottom panel), iii) STAT1 (or STAT3) binding and unbinding to dimer (Supp. Fig. 3c and 3d), iv) STAT1 (or STAT3) phosphorylation when bound to dimer (Supp. Fig. 3c and 3d), v) internalisation/degradation of complexes (Supp. Fig. 3e and 3f), and vi) dephosphorylation of free STAT1 (or STAT3) (Supp. Fig. 3g). Details of model assumptions, model parameters and parameter inference have been provided in the Material and Methods under Mathematical models and Bayesian inference.

We first wanted to explore if there existed a potential feedback mechanism in the way in which receptor molecules are internalised/degraded over time. To this end, and for each cytokine model, we considered two hypotheses: hypothesis 1 assumes that receptor complexes (Supp. Fig. 3e and 3f) are internalised with rate proportional to the concentration of the species in which they are contained (e.g., different dimer types), and hypothesis 2, that receptor complexes are internalised with rate proportional to the product of the concentration of the species in which they are contained and the sum of the concentrations of free phosphorylated STAT1 and STAT3. Hypothesis 2 is consistent with a negative feedback mechanism in which pSTAT molecules translocate to the nucleus, where they increase the production of negative feedback proteins such as SOCS3. As described in the Material and Methods (Mathematical models and Bayesian inference) we made use of the RPE1 experimental data set to carry out mathematical model selection for the two different hypotheses. We found that hypothesis 1 could explain the data better than hypothesis 2 , with a probability of $70 \%$. This result can be seen in Figure $2 \mathrm{~b}$, in which we plot, for different values of the distance threshold between the mathematical model output and the data (see Mathematical models and Bayesian inference in Material and Methods, for details), the relative probability of each hypothesis, where hypothesis 1 is denoted $H_{1}$ and hypothesis 2 is denoted $\mathrm{H}_{2}$. It can be observed that for smaller values of the distance threshold, which indicate better support from the data to the mathematical model, the relative probability of hypothesis 1 is higher than that of hypothesis 2.

We then made use of this result to explore the mathematical models for both cytokines under hypothesis 1, in particular we performed parameter calibration. To this end (and as described in Material and Methods under Mathematical models and Bayesian inference), we carried out Bayesian inference together with the mathematical models (hypothesis 1) and the experimental data sets to quantify the reaction rates (see Supp. Fig. 3) and initial molecular concentrations (see Table 1 and Table 2). The Bayesian parameter calibration of the two models of cytokine signaling allows one to quantify the observed kinetics of pSTAT1/3 phosphorylation induced by HyplL-6 and IL-27 in RPE1 and Th-1 cells (Figure 2c). Substantial differences in STAT association rates to and dissociation rates from the dimeric complexes were inferred to critically contribute to defining PSTAT1/3 kinetics. Figure $2 \mathrm{~d}$ shows the kernel density estimates (KDEs) for the posterior distributions of the rate constants and initial concentrations in the models. $k_{i a}^{+}$denotes the rate at which STAT $i$ binds to GP130 and $k_{i b}^{+}$ denotes the rate at which STAT $i$ binds to IL-27R $\alpha$, for $i \in\{1,3\}$. Our results indicate that STAT1 and STAT3 exhibit different binding preferences towards IL-27R $\alpha$ and GP130, respectively. While STAT1 exhibits stronger binding to IL-27R $\alpha$ than GP130 $\left(k_{1 b}^{+}>k_{1 a}^{+}\right)$, STAT3 exhibits stronger binding to GP130 than IL-27R $\alpha,\left(k_{3 a}^{+}>k_{3 b}^{+}\right)$in agreement with previous observations (42). 


\section{IL-27Ra cytoplasmic domain is required for sustained pSTAT1 kinetics}

The Bayesian inference carried out with the experimental data and the mathematical models clearly indicated statistically significant differences in the binding rates of STAT1/STAT3 to GP130 and IL-27R $\alpha$, to account for the different phosphorylation kinetics exhibited by HyplL6 and IL-27. Thus, we next investigated whether the more sustained STAT1 activation by IL27 resulted from its specific engagement of IL-27R $\alpha$. For that, we used RPE1 cells, which do not express IL-27R $\alpha$ (Supp. Fig. 1e), to systematically dissect the contribution of the IL-27R $\alpha$ cytoplasmic domain to the differential pSTAT activation by IL-27. IL-27R $\alpha$ 's intracellular domain is very short and only encodes two Tyr susceptible to be phosphorylated in response to IL-27 stimulation, i.e., Tyr543 and Ty613 (Figure 3a). We mutated these two Tyr to Phe to analyse their contribution to IL-27 induced signaling. We stably expressed WT IL-27R $\alpha$ as well as different IL-27R $\alpha$ Tyr mutants in RPE1 cells with comparable cell surface expression levels (Figure $3 b$ ). Importantly, this reconstituted experimental system mimicked the pSTAT1/3 activation kinetics of T-cells (Supp. Fig. 4a). As the endogenous GP130 expression levels remain unaltered, all generated clones exhibited very comparable responses to HyplL6 (Figure 3b, bottom panels). IL-27 triggered comparable levels of STAT1 and STAT3 activation in RPE1 cells reconstituted with IL-27R $\alpha$ WT and IL-27R $\alpha$ Y543F mutant, suggesting that this Tyr residue does not contribute to signaling by this cytokine (Figure $3 \mathrm{~b}$ and Supp. Fig. 4b). In RPE1 cells reconstituted with the IL-27R $\alpha$ Y613F or Y543F-Y613F mutants, IL-27 stimulation resulted in $80 \%$ of the STAT3 activation, but only $20 \%$ of the STAT1 activation levels induced by this cytokine relative to IL-27R $\alpha$ WT (Figure 3b) (43). These observations suggest a tight coupling of STAT phosphorylation to one of the receptor chains; namely, IL-27R $\alpha$ with pSTAT1 and GP130 with pSTAT3, respectively. We next tested how the cytoplasmic domains of GP130 and IL-27R $\alpha$ shape the pSTAT kinetic profiles. Thus, we generated a stable RPE1 clone expressing a chimeric construct comprised of the extracellular and transmembrane domain of IL-27R $\alpha$ but the cytoplasmic domain of GP130 (Figure 3c, Supp. Fig. 5a). Again, as both cell lines express unaltered endogenous GP130 levels, they exhibited comparable responses to HylL-6 (Figure 3c). Strikingly, this domain-swap resulted in a transient pSTAT1 kinetic response by IL-27 comparable to HyplL-6 stimulation. STAT3 activation on the other hand remained unaltered suggesting that the cytoplasmic domain of IL-27R $\alpha$ is essential for a sustained pSTAT1 response but not for pSTAT3.

Two plausible scenarios could explain the observed pSTAT1/3 activation differential by HyplL6 and IL-27: i) IL-27R $\alpha-J A K 2$ complex phosphorylates STAT1 faster than GP130-JAK1 complex or ii) pSTAT1 is more quickly dephosphorylated in the IL-6/GP130 receptor homodimer. In the latter case, pSTAT deactivation by constitutively expressed phosphatases could be an additional factor of regulation. Indeed, SHP-2 has been described to bind to GP130 and shape IL-6 responses (44). However, our Bayesian inference results (together with the mathematical models and the experimental data) identified the STAT/receptor association rates as the only rates that could account for the greater and more sustained activation of STAT1 by IL-27. We note (as described in the Material and Methods) that the phosphorylation rate, denoted by q, of STAT1 and STAT3 when bound to a dimer (homo- or hetero-) has been assumed to be independent of the STAT type and the receptor chain. Moreover, the model also included dephosphorylation of free pSTAT molecules, and predicted that the rates at which these reactions occur $\left(d_{1}\right.$ and $\left.d_{3}\right)$ had rather similar posterior distributions, hence arguing against the potential role of phosphatases to specifically target STAT1 upon HyplL-6 stimulation. To distinguish between the two plausible scenarios, we next 
determined the rates of pSTAT1/3 dephosphorylation by blocking JAK activity upon cytokine stimulation making use of the JAK inhibitor Tofacitinib in RPE1 cells. Tofacitinib was added 15 minutes after stimulation with either cytokine and PSTAT1 and PSTAT3 levels were measured at the indicated times. JAK inhibition markedly shortened the pSTAT1/3 activation profiles induced by both cytokines (Figure 3d, Supp. Fig. 5b). The relative dephosphorylation rates could then be determined by the signal intensity ratio of $+/-$ Tofacitinib. Even though pSTAT1 levels were more affected by JAK inhibition than those of pSTAT3, the observed relative changes were nearly identical for IL-27 and HyplL-6. These findings were also confirmed for Th-1 cells (Supp. Fig. $5 c$ \& 5 d) and indicate, that selective phosphatase activity cannot serve as an explanation for the pSTAT1/3 differential by HyplL-6 and IL-27, in agreement with our mathematical modelling predictions. Similarly, we tested whether neosynthesis of feedback inhibitors such as SOCS3 (19) would selectively impair signaling by HyplL-6 but not by IL-27. To this end we pre-treated cells with Cycloheximide (CHX) and followed the pSTAT1/3 kinetics induced by the two cytokines (Supp. Fig. 6a \& 6b). CHX treatment resulted in more sustained pSTAT3 activity for both cytokines. To our surprise, STAT1 phosphorylation by IL-27 was even more sustained while PSTAT1 levels induced by IL-6 remained unaffected. These observations exclude that feedback inhibitors selectively impair STAT1 activation kinetics by HyplL-6 and thus do not account for the faster STAT1 dephosphorylation kinetics observed under HyplL-6 stimulation. Overall our data from the chimera and mutant experiments, which were not used in the Bayesian calibration, provide strong and independent support, as well as validation, to the mathematical models of HyplL6 and IL-27 signaling, and point to the differential association/dissociation of STAT1 and STAT3 to IL-27R $\alpha$ and GP130, respectively, as the main factor defining STAT phosphorylation kinetics in response to HyplL-6 and IL-27 stimulation.

\section{Unique and overlapping effects of IL-27 and HyplL-6 on the Th-1 phosphoproteome}

Thus far, we have investigated the differential activation of STAT1/STAT3 induced by HypIL6 and IL-27. Next, we asked whether IL-27 and IL-6 induced the activation of additional and specific intracellular signaling programs that could contribute to their unique biological profiles. To this end, we investigated the IL-27 and HyplL-6 activated signalosome using quantitative mass-spectrometry-based phospho-proteomics. MACS-isolated CD4+ were polarized into Th1 cells and expanded in vitro for stable isotope labelling by amino acids in cell culture (SILAC). Cells were then stimulated for 15 min with saturating concentrations of IL-27, HyplL-6 or left untreated. Samples were enriched for phosphopeptides (Ti-IMAC), subjected to mass spectrometry and raw files analysed by MaxQuant software (Supp. Fig. 7a). In total we could quantify $\sim 6400$ phosphopeptides from 2600 proteins, identified across all conditions (unstimulated, IL-27, HyplL-6) for at least two out of three tested donors. For IL-27 and HyplL6 we detected similar numbers of significantly upregulated ( $87 \mathrm{vs.} 78$ ) and downregulated (155 vs. 140) phosphorylation events (Figure $4 a$ ) and systematically categorized them in context with their cellular location and ascribed biological functions (Supp. Fig. 7b \& 7c) (45). The two cytokines shared approximately half of the upregulated and one third of the downregulated phospho-peptides (Supp. Fig. 8a) but also exhibited differential target phosphorylation (Figure $4 \mathrm{~b}$ and Supp. Fig. 8b). As expected, we found multiple members of the STAT protein family among the top phosphorylation hits by the two cytokines, validating our study (Figure $4 \mathrm{~b} \& 4 \mathrm{c}$ ). In line with our previous observations, we detected the same relative amplitudes for tyrosine phosphorylated STAT3 and STAT1. In addition to tyrosinephosphorylation, we detected robust serine-phosphorylation on S727 for STAT1 and STAT3 (Figure 4c). While pS-STAT1 activity correlated with pY-STAT1 with IL-27 being more potent 
than HyplL-6, this was not the case for STAT3. Despite an identical pY-STAT3 phosphorylation profile, HyplL-6 induced a $\sim 50 \%$ higher pS-STAT3 relative to IL-27 (Figure 4c). These results were corroborated, following the phosphorylation kinetics of pSSTAT1 and pS-STAT3 by flow-cytometry (Figure 4d).

Given the overlapping phospho-proteomic changes, gene ontology (GO) analysis associated several sets of phosphopeptides with biological processes that were mostly shared between both cytokines (Figure 4e, Supp. Fig. 8c). A large set of phospho-peptides was linked to transcription initiation (including JAK/STAT signaling) or mRNA modification (Figure 5e). Interestingly, IL-27 stimulation was associated to negative regulation of RNA polymerase II, whereas a positive regulation was detected for HyplL-6. A closer look into the functional regulation of RNA-pol II activity by the two cytokines revealed that multiple proteins involved in this process were differentially regulated by HyplL-6 and IL-27 (Figure 5f). While positive regulators of RNA-pol II transcription, such as Negative Elongation Factor A (NELFA), PPM1G, RCHY1 and POL2RA, were much more phosphorylated in response to HyplL-6 than IL-27, negative regulators of RNA-pol II transcription, such as LARP7, were much more engaged by IL-27 treatment than by HyplL-6 (Figure 4f). Interestingly, in a previous study we linked RNA-pol II regulation with the levels of STAT3 S727phosphorylation induced by HyplL6 via recruitment of CDK8 to STAT3 dependent genes (46). Our phospho-proteomic analysis thus, suggests that IL-27 and HyplL-6 recruit different transcriptional complexes that ultimately could contribute to provide gene expression specificity by the two cytokines. Additionally, we identified several interesting IL-27-specific phosphorylation targets. One example was Ubiquitin Protein Ligase E3 Component N-Recognin 5 (UBR5). Phosphorylated UBR5 leads to ubiquitination and subsequent degradation of Ror $\gamma \mathrm{c}$ (47), the key transcription factor required for Th-17 lineage commitment, thus limiting Th-17 differentiation (Supp. Fig. 8d). A second example is PAK2, which phosphorylates and stabilizes FoxP3 leading to higher levels of $\mathrm{T}_{\text {Reg }}$ cells (Supp. Fig. 8d) (48). Moreover, IL-27 stimulation led to a very strong phosphorylation of BCL2-associated agonist of cell death (BAD), a critical regulator of T-cell survival and a well-known substrate of the PAK2 kinase (49). Overall, our data show a large overlap between the IL- 6 and IL-27 signaling program, with a strong focus on JAK/STAT signaling. However, IL-27 engages additional signaling intermediaries that could contribute to its unique immuno-modulatory activities. Further studies will be required to assess how these IL-27 specific signaling pockets contribute to shape IL-27 responses.

\section{Kinetic decoupling of gene induction programs depends on sustained STAT1 activation and IRF1 expression by IL-27}

Next, we investigated how the different kinetics of STAT activation induced by HyplL-6 and IL-27 ultimately modulated gene expression by these two cytokines. To this end, we performed RNA-seq analysis of Th-1 cells stimulated with HyplL-6 or IL-27 for $1 \mathrm{~h}, 6 \mathrm{~h}$ and $24 \mathrm{~h}$ to obtain a dynamic perspective of gene regulation. We identified $\sim 12500$ shared genes that could be quantified for all three donors and throughout all tested experimental conditions. In a first step, we compared how similar the gene programs induced by HyplL-6 and IL-27 were. Principal component analysis (PCA) was run for a subset of genes, found to be significantly up(total $\sim 250$ ) or downregulated (total $\sim 950$ ) by either of the experimental conditions ( $p$ value $\leq$ 0.05 , fold change $\geq+2$ or $\leq-2$ ). At one hour of stimulation HyplL-6 and IL-27 induced very similar gene programs, with the two cytokines clustering together in the PCA analysis regardless of whether we focused on the subsets of upregulated or downregulated genes (Figure 5a). However, the similarities between the two cytokines changed dramatically in the 
course of continuous stimulation. While the two cytokines induced the downregulation of comparable gene programs at $6 \mathrm{~h}$ and $24 \mathrm{~h}$ stimulation, as denoted by the close clustering in the PCA analysis (Figure 5a, right panel) and the fraction of shared genes ( $40 \%$, Figure $5 \mathrm{~b}$, Supp. Fig. 9a-c, Supp. Fig. 10a), this was not observed for upregulated genes. Although the two cytokines induced comparable gene upregulation programs after $1 \mathrm{~h}$ of stimulation $(\sim 80 \%$ shared genes), this trend almost completely disappeared at later stimulation times (Figure 5a \& 5b, Supp. Fig. 10b). This is well-reflected by the absolute numbers of up- or downregulated genes observed for IL-27 and HyplL-6 (Figure 5c). Stimulation with both cytokines yielded a similar trend of gene downregulation (Figure $5 \mathrm{c}$, right panel). However, while HyplL-6 stimulation resulted in a spike of gene upregulation at $1 \mathrm{~h}$ that quickly disappeared at later stimulation times, IL-27 stimulation was capable to increase the number of upregulated genes beyond $6 \mathrm{~h}$ of stimulation and maintains it even after $24 \mathrm{~h}$ (Figure $5 \mathrm{c}$, left panel). This "kinetic decoupling" of gene induction seems to have a striking functional relevance. Gene set enrichment analysis (GSEA) (50) identified several reactome pathways to be enriched for IL-27 over the course of stimulation - most of them linked with Interferon signaling and immune responses (Figure 5d). In contrast, for HyplL-6 stimulation no pathway enrichment was detected. Most importantly, the vast majority of IL-27-induced genes that were associated to these pathways belonged to genes upregulated by IL-27 treatment and that have been previously linked to STAT1 activation $(51,52)$ (Supp. Fig. 10c). Although HyplL-6 treatment resulted in the induction of some of these genes, their expression was very transient in time, in agreement with the short STAT1 activation kinetic profile exhibited by HyplL-6 (Supp. Fig. 10b \& 10c).

Next, we performed cluster analysis to find further similarities and discrepancies between the gene expression programs engaged by HyplL-6 and IL-27 (Figure 5e). Since genes downregulated by IL-27 and HyplL-6 showed overall good similarity throughout the whole kinetic series, we mainly focused on differences in upregulated gene induction. We identified three functionally relevant gene clusters. The first gene cluster corresponds to genes that are transiently and equally induced by HyplL-6 and IL-27. These genes peak after one hour and return to basal levels after $6 \mathrm{~h}$ and $24 \mathrm{~h}$ of stimulation (Figure $5 \mathrm{e}$ ). Interestingly, this cluster contains classical IL-6-induced and STAT3-dependent genes, such as members of the NFKB and Jun/Fos transcriptional complex (53), as well as the feedback inhibitor Suppressor Of Cytokine Signaling 3 (SOCS3) (54) and T-cell early activation marker CD69. (Figure 5e). A second cluster of genes corresponded to genes that were persistently activated by IL-27 but only transiently by HyplL-6 (Figure 5e). Among these genes we found classical STAT1dependent genes, such as SOCS1, Programmed Cell Death Ligand $1(P D L 1=C D 274)(55)$ and members of the interferon-induced protein with tetratricopeptide repeats (IFIT) family. The third cluster of genes corresponded to genes exhibiting strong and sustained activation by IL27 after $6 \mathrm{~h}$ and $24 \mathrm{~h}$ stimulation but no activation by HyplL-6 at all. This " ${ }^{\text {nd }}$ wave" of gene induction by IL-27 was almost exclusively comprised of classical Interferon Stimulated Genes (ISGs) (Supp. Fig. 10c), such as STAT1 \& 2, Guanylate Binding Protein 1 (GBP1), GBP2, 4 \& 5, and IRF8 \& 9 .

It is worth mentioning, that genes in the third cluster appear to require persistent STAT1 activation $(56,57)$ and were the basis for the IFN signature identified in our reactome pathway analysis. Still, we were surprised about the magnitude of this $2^{\text {nd }}$ gene wave. Even though IL27 exerts a sustained pSTAT1 kinetic profile, pSTAT1 levels were down to $\sim 10 \%$ of maximal amplitude after $3 \mathrm{~h}$ of stimulation. We reasoned that additional factors could further amplify the STAT1 response for IL-27 but not for HypIL-6. Within the $1^{\text {st }}$ wave of STAT1-dependent genes, 
we also spotted the transcription factor Interferon Response Factor 1 (IRF1), that was continuously induced throughout the kinetic series in response to IL-27 but only transiently spiking after $1 \mathrm{~h}$ of HyplL-6 stimulation (Figure 5e). IRF1 expression was shown to prolong pSTAT1 kinetics (58) and to be required for IL-27-dependent Tr-1 differentiation and function (59). We confirmed the kinetics of IRF1 protein expression by flow cytometry and showed higher and more sustained protein levels after IL-27 stimulation relative to HyplL-6 (Figure 6a). Next, we tested in our RPE1 cell system, whether siRNA mediated knockdown of IRF1 would alter the gene induction profiles of certain STAT1 or STAT3-dependent marker genes. In RPE1 cells, reconstituted with IL-27R $\alpha$, IRF1 protein levels were peaking around $6 \mathrm{~h}$ after stimulation with IL-27 and transfection with IRF1-targeting siRNA knocked down expression by $>80 \%$ (Figure $6 \mathrm{~b}$ ). Importantly, knockdown of IRF1 did not alter the overall kinetics of pSTAT1 and pSTAT3 activation (Figure 6c). Induction of STAT1-dependent genes STAT1, GBP5 and OAS1 as well as STAT3-dependent gene SOCS3 were followed by RT qPCR (Figure $6 \mathrm{~d}$ ). Interestingly, up to $6 \mathrm{~h}$ of stimulation, the gene induction curves were identical for control- and IRF1-siRNA treated cells. Later than $6 \mathrm{~h}$ - that is, when IRF1 protein levels are peaking - the gene induction was decreased between $40-70 \%$ in absence of IRF1. Strikingly, expression of SOCS3, a classical STAT3-dependent reporter gene was transient and independent on IRF1 levels, highlighting that IRF1 selectively amplifies STAT1-dependent gene induction. Taken together our data support a scenario whereby IL-27 by exhibiting a kinetic decoupling of STAT1 and STAT3 activation is capable of triggering independent gene expression waves, which ultimately contribute to shape its distinct biology.

\section{IL-27-induced STAT1 response drives global proteomic changes in Th-1 cells}

Next, we aimed to uncover how the distinct gene expression programs engaged by HyplL-6 and IL-27 ultimately relate to alterations of the Th-1 cell proteome. For that, we continuously stimulated SILAC labelled Th-1 cells for $24 \mathrm{~h}$ with saturating doses of IL-27 and HypIL-6 and compared quantitative proteomic changes to unstimulated controls (Figure 7a). We quantified $\sim 3600$ proteins present in all three biological replicates and in all tested conditions (unstimulated/IL-27/HyplL-6). Both cytokines downregulated a similar number of proteins (IL27: 57, HyplL-6: 52) (Figure 7b) with approximately half of them being shared by the two cytokines, mimicking our observations in the RNA-seq studies (Figure 7c, Supp. Fig. 11a). With 68 upregulated proteins, IL-27 was almost twice as potent as HyplL-6 (35 proteins) with very little overlap.

Among the upregulated proteins by IL-27 but not HyplL-6, we detected several proteins with described immune-modulatory functions on T-cells. One of these proteins was Transforming Growth Factor $\beta$ (TGF- $\beta$ ), which is a key regulator with pleiotropic functions on T-cells $(60)$. TGF- $\beta$ has been identified to synergistically act with IL-27 to induce IL-10 secretion from Tr-1 cells - thus accounting for one of the key anti-inflammatory functions of IL-27 (61). On the other hand, we also found SELPLG-encoded protein RSGL-1 which is critically required for efficient migration and adhesion of Th-1 cells to inflamed intestines $(62,63)$. Interestingly, we found LARP7 moderately upregulated by IL-27. This negative regulator for RNA pol II was also identified in our phospho-target screening and selectively engaged by IL-27 (Figure 4f). IL-27 and HyplL-6 share $\sim 60 \%$ of downregulated proteins, but without strong functional patterns. Both cytokines downregulated several proteins related to mitotic cell cycle (LIG1, CSNK2B, PSMB1) mRNA processing and splicing (NCBP2, PCBP2, NUDT21) (64).

Strikingly, a significant number ( $40 \%)$ of proteins upregulated by IL-27 belong to the group of ISGs (Figure 7b \& 7c, Supp. Fig. 11b). This particular set of proteins including STAT1, 
STAT2, MX Dynamin like GTPase 1 (MX1), Interferon Stimulated Gene 20 (ISG20) or Poly(ADP-Ribose) Polymerase Family Member 9 (PARP9) was not markedly altered by HyplL-6. Of note: the overall expression patterns of the most significantly altered proteins are congruent to the gene induction patterns observed after 6h and 24h (Figure 7d \& 7e, Supp. Fig. 10b). Similar to this, GSEA reactome analysis identified again pathways associated with interferon signaling and cytokine/immune system but failed to detect any significant functional enrichment by HyplL-6 (Figure 7e, Supp. Fig. 11b \& 11c). Finally, we correlated RNAseq-based gene induction patterns with detected proteomic changes. To our surprise we only found a relatively low number of shared hits. However, the identified proteins belong exclusively to a group upregulated by IL-27 (Figure 7f). They are all located in the " $2^{\text {nd }}$ gene wave" cluster and all of them are regulated by ISGs (Figure 5e). Taken together these results provide compelling evidence that sustained pSTAT1 activation by IL-27 accounts for its gene induction and proteomic profiles, thus, giving a mechanistic explanation for the diverse biological outcomes of IL-27 and IL-6. Our observations are in good agreement with previous findings in cancer cells, showing that particularly the involvement of STAT1 activation is responsible for proteomic remodeling by IL-27 (65).

\section{Receptor and STAT concentrations determine the nature of the IL-6/IL-27 response}

Our data suggest that STAT molecules compete for binding to a limited number of phosphoTyr motifs in the intracellular domains of cytokine receptors. A direct consequence derived from this hypothesis is that cells can adjust and change their responses to cytokines by altering their concentrations of specific STATs or receptors molecules. To assess to what degree immune cells differ in their expression of cytokine receptors and STATs, we investigated levels of IL-6R $\alpha$, GP130, IL-27R $\alpha$, STAT1 and STAT3 protein expression across different immune cell populations making use of the Immunological Proteomic Resource (ImmPRes http://immpres.co.uk) database. Strikingly, the level of expression of these proteins change dramatically across the populations studied (Figure 8a), suggesting that these cells could potentially produce very different responses to HyplL-6 and IL-27 stimulation.

In order to quantify (and predict) how changes in expression levels of different proteins modify the kinetics of pSTAT, we made use of the two mathematical models of HypIL-6 and IL-27 stimulation and the parameters inferred with Bayesian methods. Our mathematical models could accurately reproduce the experimental results generated across our study, i.e., signaling by the IL-27R $\alpha$ chimeric and IL-27R $\alpha$-Y616F mutant receptors and dose/response studies (Supp. Fig. 12a-c), making use of the posterior parameter distributions generated from the Bayesian parameter calibration. Having developed mathematical models which are able to accurately explain the experimental data (Supp. Fig. $5 b$ and $5 c$ ) and reproduce independent experiments (Fig. 3b and 3c), we then sought to use the models to predict pSTAT signaling kinetics under different concentration regimes of receptors and STATs. To simplify the simulations, we focused our analysis in GP130 and STAT1 proteins, two of the proteins that greatly vary in the different immune populations (Figure 8a). As baseline values for the concentrations [GP130(0)], [IL27Ra(0)] [STAT1(0)] and [STAT3(0)] we used approximately the median values from the posterior distributions for each parameter: $[G P 130(0)]=25 \mathrm{nM}$, $[\operatorname{IL27Ra(0)}]=50 \mathrm{nM}$ and $[S T A T 1(0)]=[S T A T 3(0)]=500 \mathrm{nM}$. To see the effect of varying GP130 concentrations on pSTAT signaling, we decreased the initial concentration of GP130 and simulated the model using the accepted parameters sets from the ABC-SMC to inform the other parameter values. A tenfold reduction on GP130 concentration $([G P 130(0)]=$ 2.5 $\mathrm{nM}$ ) resulted in a striking loss in pSTAT1 levels induced by HyplL-6, with very little effect 
on pSTAT3 levels induced by this cytokine (Figure 8b). pSTAT1/3 kinetics induced by IL-27 however was not affected by this decrease in GP130 concentration (Figure 8b). Interestingly, the HyplL-6 signaling profile predicted by our model at low GP130 concentrations strongly resemble the one induced by HyplL-6 in Th-1 cells (Figure 1c), where very low levels of GP130 are found, further confirming the robustness of the predictions generated by our mathematical models. When the concentration of STAT1 was increased by a factor of ten $([\operatorname{STAT} 1(0)]=$ $5000 \mathrm{nM}$, both HyplL-6 and IL-27 induced significantly higher levels of pSTAT1 activation (Figure 8b). pSTAT3 levels were not affected for HyplL-6 stimulation but were decreased for IL-27 stimulation (Figure 8b), further indicating the competitive nature of the binding of STAT1 and STAT3 to IL-27R $\alpha$ and GP130. Overall, our mathematical model predicts that changes on GP130 and STAT1 expression produce a substantial remodeling of the HypIL-6 and IL-27 signalosome, which ultimately could lead to aberrant responses.

\section{STAT1 protein levels in SLE patients modify HypIL-6 and IL-27 signaling responses}

STAT1 is a classical IFN responsive gene and STAT1 levels are highly increased in environments rich in IFNs (66). Thus, we next ask whether STAT1 levels would be increased in SLE patients, an examples of disease where IFNs have been shown to correlate with a poor prognosis, making use of available gene expression datasets (67). We did not find differences in the expression of GP130, IL-6R $\alpha$ or IL-27R $\alpha$ in SLE patients (Figure 8c). However, we detected a significant increase in the levels of STAT1 and STAT3 transcripts in these patients when compared to healthy controls, with the increase on STAT1 expression being significantly more pronounced (Figure 8c). Since our mathematical model predicted that increases in STAT1 expression could significantly change cytokine-induced cellular responses by HyplL-6 and IL-27, we next experimentally tested this prediction. For that, we primed Th-1 cells with IFN $\alpha 2$ overnight to increase total STAT1 levels (and to a lower extent STAT3) in these cells (Supp. Fig. 13a). While both HyplL-6 and IL-27 induced comparable levels of pSTAT3 in primed and non-primed Th-1 cells, levels of pSTAT1 induced by the two cytokines were significantly upregulated in primed Th-1 cells, resulting in a bias STAT1 response and confirming our model predictions (Figure 8d). We next investigated whether this bias STAT1 activation by HyplL- 6 and IL-27 observed in IFN $\alpha 2$-primed Th-1 cells was also present in SLE patients. For that we collected PBMCs from six SLE patients or five age-matched healthy controls and measured STAT1 and STAT3 expression, as well as pSTAT1 and pSTAT3 induction by HylL-6 and IL-27 after 15 min treatments in CD4 T cells. Importantly, comparable results to those obtained with IFN-primed Th-1 cells were obtained, with signaling bias towards pSTAT1 in CD4+ T cells from SLE patients stimulated with HyplL-6 and IL-27 (Figure 8e, Supp. Fig. $13 b \& c$ ), further supporting the fact that STAT concentrations play a critical role in defining cytokine responses in autoimmune disorders.

Our data show that STAT1 and STAT3 compete for phospho-Tyr motifs in GP130, with STAT3 having an advantage resulting from its tighter affinity to GP130. Finally, we asked whether crippling JAK activity by using sub-saturating doses of JAK inhibitors could differentially affect STAT1 and STAT3 activation by HypIL-6 and therefore rescue the altered cytokine responses found in SLE patients. To test this, RPE1 and Th-1 cells were stimulated with saturated concentrations of HyplL- 6 and titrating the concentrations of Tofacitinib, a clinically approved JAK inhibitor. Strikingly, Tofacitinib inhibited HyplL-6 induced pSTAT1 more efficiently than pSTAT3 in both RPE1 cells and Th-1 cells (Figure 8f). At $50 \mathrm{nM}$ concentration, Tofacitinib inhibited pSTAT1 levels induced by HyplL- 6 by $60 \%$, while only inhibited pSTAT3 levels by $30 \%$ (Figure 8f) - an effect that we did not observe for IL-27 stimulation (Supp. Fig. 13d). 
bioRxiv preprint doi: https://doi.org/10.1101/2021.01.08.425379; this version posted January $9,2021$. The copyright holder for this preprint (which was not certified by peer review) is the author/funder, who has granted bioRxiv a license to display the preprint in perpetuity. It is made available under aCC-BY 4.0 International license.

Overall, our results show that the changes in STATs concentration found in autoimmune disorders shape cytokine signaling responses and could contribute to disease progression. 


\section{DISCUSSION:}

Cytokine pleiotropy is the ability of a cytokine to exert a wide range of biological responses in different cell types. This functional pleiotropy has made the study of cytokine biology extremely challenging given the strong cross-talk and shared usage of key components of their signaling pathways, leading to a high degree of signaling plasticity, yet still allowing functional selectivity $(68,69)$. Here we aimed to identify the underlying determinants that define cytokine functional selectivity by comparing IL-27 and IL-6 at multiple scales - ranging from cell surface receptors to proteomic changes. We show that IL-27 triggers a more sustained STAT1 phosphorylation than IL-6, via a high affinity STAT1/IL-27R $\alpha$ interaction centered around Tyr613 on IL-27R $\alpha$. This in turn results in a more sustained IRF1 expression induced by IL-27, which leads to the upregulation of a second wave of gene expression unique to IL-27 and comprised of classical ISGs. We go one step further and show that this strong receptor/STAT coupling is altered in autoimmune disorders where STATs concentrations are often dysregulated. Increased expression of STAT1 in SLE patients biases HypIL-6 and IL-27 responses towards STAT1 activation, further contributing to the worsening of the disease. By using suboptimal doses of the JAK inhibitor Tofacitinib we show that specific STAT proteins engaged by a given cytokine can be targeted. Overall, our study highlights a new layer of cytokine signaling regulation, whereby STAT affinity to specific cytokine receptor phospho-Tyr motifs controls STAT phosphorylation kinetics and the identity of the gene expression program engaged, ultimately ensuing the generation of functional diversity through the use of a limited set of signaling intermediaries.

The tight coupling of one receptor subunit to one particular STAT that we have identified in our study is a rather unusual phenomenon for heterodimeric cytokine receptor complexes, which has been first suggested by Owaki et al. (27). Generally, the entire signaling output driven by a cytokine-receptor complex emanates from a dominant receptor subunit, which carries several Tyr residues susceptible of being phosphorylated $(70,71)$. This in turn results in competition between different STATs for binding to shared phospho-Tyr motifs in the dominant receptor chain, leading to different kinetics of STAT phosphorylation as observed for IL-6 stimulation (15) (Figure 1b). Moreover, this localized signaling quantum allows phosphatases and feedback regulators - induced upon cytokine stimulation - to act in synergy to reset the system to its basal state, generating a very synchronous and coordinated signaling wave. Although very effective, this molecular paradigm presents its limitations. STAT competition for the same pool of phospho-Tyr makes the system very sensitive to changes in STAT concentration. IFN $\gamma$ primed cells, which exhibit increased STAT1 levels, trigger an IFN $\gamma$ like STAT1 response upon IL-6 stimulation (16). IL-10 anti-inflammatory properties are lost in cells with high levels of STAT1 expression, as a result of a pro-inflammatory environment rich in IFNs (72). Indeed, we show that STAT1 transcripts levels are increased in Crohn's disease and SLE patients and they contributed to alter IL-6 responses. Strikingly, IL-27 appears to have evolved away from this general model of cytokine signaling activation. Our results show that STAT1 activation by IL-27 is tightly coupled to IL-27R $\alpha$, while STAT3 activation by this cytokine mostly depends on GP130. This decoupled STAT1 and STAT3 activation by IL-27 is possible thanks to the presence of a putative high affinity STAT1 binding site on IL-27R $\alpha$ that resembles the one present in IFN $\gamma \mathrm{R} 1$ (41). As a result of this, IL-27 can trigger sustained and independent phosphorylation of both STAT1 and STAT3. This unique feature of IL-27 allows it to induce robust responses in dynamic immune environments. Indeed, our mathematical models of cytokine signaling and Bayesian inference, together with the experimental observations show that changes in receptor concentration minimally affected 
pSTAT1/3 induced by IL-27, while they fundamentally alter IL-6 responses. Overall, our data show that cytokine responses are versatile and adapt to the continuously changing cell proteome, highlighting the need to measure cytokine receptors and STATs expression levels, in addition to cytokine levels, in disease environments to better understand and predict altered responses elicited by dysregulated cytokines.

In recent years, it has become apparent that the stability of the cytokine-receptor complex influences signaling identity by cytokines (73). Short-lived complexes activate less efficiently those STAT molecules that bind with low affinity phospho-Tyr motif in a given cytokine receptor (34). Our current results further support this kinetic discrimination mechanism for STAT activation. Our statistical inference identified differences in STAT recognition to the cytokine receptor phospho-Tyr motifs as one of the major determinants of STAT phosphorylation kinetics. This parameter alone was sufficient to explain transient and sustained STAT1 phosphorylation induced by IL-6 and IL-27, respectively, without the need to invoke the action of phosphatases or negative feedback regulators such as SOCSs. Indeed, our results indicate that the rate of STAT1 dephosphorylation is similar between the IL-6 and IL-27 systems, suggesting that phosphatases do not contribute to these early kinetic differences. Moreover, blocking protein translation, and therefore the upregulation of negative feedback regulators by IL-6 treatment did not result in a more sustained STAT1 phosphorylation by IL-6, again indicating that the transient kinetics of STAT1 phosphorylation by IL- 6 is encoded at the receptor level and does not require further regulation. However, recent reports have found that the amplitude of STAT1 phosphorylation in response to IL-6 is regulated by levels of PTPN2 expression, suggesting that phosphatases can play additional roles in shaping IL-6 responses beyond controlling the kinetics of STAT activation (74). STAT1 phosphorylation levels by IL-27 on the other hand were significantly more sustained in the absence of protein translation, suggesting that negative feedback mechanisms are required to downmodulate signaling emanating from high affinity STAT-receptor interactions. Overall our results suggest that while phosphatases and negative feedback regulators play an important role in maintaining cytokine signaling homeostasis (75), the kinetics of STAT activation appears to be already encoded at the level of receptor engagement, thus ensuring maximal efficiency and signal robustness.

Cytokine signaling plasticity can occur at the level of receptor activation. In the past years, a scenario has emerged suggesting that the absolute number of signaling active receptor complexes is a critical determinant for signal output integration. Accordingly, specific biological responses were shown to be tuned either by abundance of cell surface receptors $(76,77)$ or by the level of receptor assembly $(34,38,78)$. Here, we show for the first time that IL-27induced dimerization of IL-27R $\alpha$ and GP130 at the cell surface of live cells - in good agreement with previous studies on heterodimeric cytokine receptor systems $(38,73)$. For IL27, the receptor subunits IL-27R $\alpha$ and GP130 can be expressed at different ratios as seen for naïve vs. activated T-cells (79) as well as intestinal cells (80). On T-cells, particularly after activation, IL-27R $\alpha$ is expressed in strong excess over GP130, rendering GP130 as the limiting factor for receptor complex assembly (41). Interestingly, we observe that in addition to a faster kinetic of STAT1 phosphorylation, HyplL-6 treatment induces a lower maximal amplitude in pSTAT1 activation in T cells. This is in stark contrast to our results in RPE1 cells, where high abundance of GP130 ( 3000-4000 copies of cell surface GP130) is found. In these cells both cytokines elicited similar amplitudes of STAT1 phosphorylation. Our results suggest that surface receptor density in synergy with STATs binding dynamics to phospho-Tyr motif 
on cytokine receptors act to define the amplitude and kinetics of STAT activation in response to cytokine stimulation.

The distinct STAT1 and STAT3 kinetic profiles induced by IL-6 and IL-27 are the prerequisite for time-correlated decoupling of genetic programs: a "shared GP130/STAT3-dependent

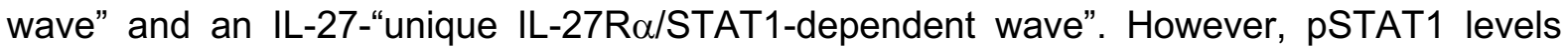
induced by IL-27 at $3 \mathrm{~h}$ were down to $\sim 10 \%$ of maximal amplitude, suggesting that additional factors would be required to amplify the initial STAT1 response elicited by IL-27. We observed that IL-27 induces the expression of an early wave of classical STAT1-dependent genes, which is also shared by IL-6. However, while IL-27 induces the upregulation of these genes throughout the entire duration of the experiment, IL-6 only resulted in a transient spike. We reasoned that this additional factor required for IL-27 signal amplification would be among these early STAT1-dependent genes. Among this set of genes we found the transcription factor IRF1, which had been shown to act as a feedback amplificant for pSTAT1 activity (58). Importantly, IRF1 protein levels have been shown to be upregulated in response to IL-27 and IFN $\gamma$ but not to IL-6 stimulation in hepatocytes (81). IRF1 plays a key role in chromatin accessibility which is critically required for IL-27-induced differentiation of Tr1 cells and subsequent IL-10 secretion (59). Here, we could prove that the contribution of IRF1 on STAT1but not STAT3-dependent genes is a generic feature of IL-27 signaling. This readily explains the significant transcriptomic overlap of IL-27 with type I (82) or type II interferons (15) after long-term stimulation with these cytokines. Along this line, it is not surprising that IL-27 beyond its well-described effects on T-cell development - can also mount a considerable antiviral response as shown in hepatic cells and PBMCs $(83,84)$. Our results suggest that by modulating the kinetics of STAT phosphorylation, cytokines can modulate the expression of accessory transcription factors, such as IRF1, that act in synergy with STATs to fine-tune gene expression and provide functional diversity. 


\section{ACKNOWLEDGMENTS}

We thank members of the Moraga, Molina-París, Piehler and Mitra laboratories for helpful advice and discussion. We thank G. Hikade and H. Kenneweg for technical support, C. P. Richter for providing software for single-molecule image analysis, R. Kurre (Integrated Bioimaging Facility Osnabrück) for support with fluorescence microscopy and the FingerPrints Proteomics facility (Dundee) for support with the mass spectrometry data. This work was supported by the StG, LS6, Wellcome-Trust-202323/Z/16/Z (IM EP), ERC-206-STG grant (IM JMF EP PKF), EMBO (SW 454-2017), DFG (SFB 944, P8/Z, JP), National Heart, Lung and Blood Institute (K22HL125593, MK) and Contrat de Plan Etat Région Hauts de France and Institut pour la Recherche sur le Cancer de Lille (SM SG). CMP and GL were supported by H2020, QuanTII. PJ is supported by the EPSRC, AstraZeneca and Smith Institute (Smith Institute CASE studentship, award reference 1969354). Numerical work was undertaken on ARC3, which is part of the High Performance Computing facilities at the University of Leeds, UK.

\section{COMPETING INTERESTS}

The authors declare that they have no competing interests. 


\section{MATERIAL AND METHODS}

\section{Protein expression and purification:}

Murine IL-27 was cloned as a linker-connected single-chain variant (p28+EBI3) as described in (29). Human HyperlL-6 (HyplL-6), and murine single-chain IL-27 were cloned into the pAcGP67-A vector (BD Biosciences) in frame with an N-terminal gp67 signal sequence and a $\mathrm{C}$-terminal hexahistidine tag, and produced using the baculovirus expression system, as described in (85). Baculovirus stocks were prepared by transfection and amplification in Spodoptera frugiperda (Sf9) cells grown in SF900II media (Invitrogen) and protein expression was carried out in suspension Trichoplusiani ni (High Five) cells grown in InsectXpress media (Lonza).

Purification was performed using the method described in (86). For IL-27, the cells were pelleted with centrifugation at $2000 \mathrm{rpm}$, prior to a precipitation step through addition of Tris $\mathrm{pH}$ 8.0, $\mathrm{CaCl}_{2}$ and $\mathrm{NiCl}_{2}$ to final concentrations of $200 \mathrm{mM}, 50 \mathrm{mM}$ and $1 \mathrm{mM}$ respectively. The precipitate formed was then removed through centrifugation at $6000 \mathrm{rpm}$. Nickel-NTA agarose beads (Qiagen) were added and the target proteins purified through batch binding followed by column washing in HBS-Hi buffer (HBS buffer supplemented to $500 \mathrm{mM} \mathrm{NaCl}$ and $5 \%$ glycerol, $\mathrm{pH}$ 7.2). Elution was performed using HBS-Hi buffer plus $200 \mathrm{mM}$ imidazole. Final purification was performed by size exclusion chromatography on an ENrich SEC 650300 column (Biorad), again equilibrated in HBS-Hi. Concentration of the purified sample was carried out using $10 \mathrm{kDa}$ Millipore Amicon-Ultra spin concentrators. For HyplL-6, proteins were purified likewise, but in $10 \mathrm{mM}$ HEPES ( $\mathrm{pH}$ 7.2) containing $150 \mathrm{mM} \mathrm{NaCl}$. Recombinant cytokines were purified to greater than $98 \%$ homogeneity.

For cell surface labeling, the anti-GFP nanobody (NB) "enhancer" and "minimizer" were used, which bind mEGFP with subnanomolar binding affinity (87). NB was cloned into pET-21a with an additional cysteine at the C-terminus for site-specific fluorophore conjugation in a 1:1 fluorophore:nanobody stoichiometry. Furthermore, (PAS $)_{5}$ sequence to increase protein stability and a His-tag for purification were fused at the C-terminus. Protein expression in $E$. coli Rosetta (DE3) and purification by immobilized metal ion affinity chromatography was carried out by standard protocols. Purified protein was dialyzed against HEPES pH 7.5 and reacted with a two-fold molar excess of DY647 maleimide (Dyomics), ATTO 643 maleimide (AT643) and ATTO Rho11 maleimide (Rho11) (ATTO-TEC GmbH), respectively. After $1 \mathrm{~h}$, a 3-fold molar excess (with respect to the maleimide) of cysteine was added to quench excess dye. Protein aggregates and free dye were subsequently removed by size exclusion chromatography (SEC). A labeling degree of 0.9-1:1 fluorophore:protein was achieved as determined by UV/Vis spectrophotometry.

\section{CD4+ T cell purification and Th-1 differentiation:}

Human buffy coats were obtained from the Scottish Blood Transfusion Service and peripheral blood mononuclear cells (PBMCs) of healthy donors were isolated from buffy coat samples by density gradient centrifugation according to manufacturer's protocols (Lymphoprep, STEMCELL Technologies). From each donor, $100 \times 10^{6}$ PBMCs were used for isolation of CD4+ T-cells. Cells were decorated with anti-CD4 ${ }^{\text {FITC }}$ antibodies (Biolegend, \#357406) and isolated by magnetic separation according to manufacturer's protocols (MACS Miltenyi) to a purity $>98 \% \mathrm{CD} 4+$. Freshly isolated resting $\mathrm{CD}^{+} \mathrm{T}$ cells $\left(3 \times 10^{7}\right.$ per donor) were activated under Th-1 polarizing conditions using ImmunoCult ${ }^{\mathrm{TM}}$ Human CD3/CD28 T Cell Activator (StemCell, Cat\#10971) following manufacturer instructions for 3 days in RPMI-1640, 10\% v/v 
FBS, $100 \mathrm{U} / \mathrm{ml}$ penicillin-streptomycin (Gibco) in the presence of the cytokines IL-2 (Novartis, \#709421, $20 \mathrm{ng} / \mathrm{ml})$, anti-IL-4 antibody (10 ng/ml, BD Biosciences, \#554481), IL-12 (20 ng/ml, BioLegend, \#573002). After three days of priming, cells were expanded for another 5 days in the presence of IL-2 $(20 \mathrm{ng} / \mathrm{ml})$.

\section{Human SLE patient samples:}

This study was authorized by the French Competent Authority dealing with Research on Human Biological Samples namely the French Ministry of Research. The Authorization number is ECH 19/04. To issue such authorization, the Ministry of Research has sought the advice of an independent ethics committee, namely the "Comité de Protection des Personnes," which voted positively, and all patients gave their written informed consent. The healthy volunteer was recruited to serve as healthy control individuals. Healthy and patients' blood samples were collected in heparinized tubes (BD Vacutainer 368886, BD Biosciences San Jose, CA, USA) and PBMC samples were isolated using Ficoll (Pancoll, Pan Biotech \#P04-60500) density gradient centrifugation. The isolated PBMCs were washed with PBS and the remaining red blood cells were lysed using RBC lysis buffer (ACK lysing buffer, Gibco \#A10492-01), incubate $3 \mathrm{~min}$ at room temperature. Cells were washed in PBS and resuspend the cells with $1 \mathrm{ml}$ of freezing medium (with DMSO, PAN Biotech, \#P07-90050) and transfer the cells in a cryotube. cryotube in a Freezing container (Nalgene) and at $-80^{\circ} \mathrm{C}$ and then transferred into liquid nitrogen container for long term storage.

\section{Classification and demographic information about SLE patients and healthy controls:}

SLE patients were included if they fulfilled the American College of Rheumatology (ACR) Classification Criteria (Hochberg MC. Updating the American College of Rheumatology revised criteria for the classification of systemic lupus erythematosus (88). Exclusion criteria were current intake of $10 \mathrm{mg}$ or more of prednisone or equivalent and/or use of immunosupressants within the previous 6 months before inclusion. Use of hydroxychloroquine was not an exclusion criterion. Patients were mostly in clinical remission, half with biological remission, half with persistent anti native DNA autoantibodies. All SLE patients and healthy controls were females between 41 and 58 years old.

\section{(Phospho-) Proteomics:}

For (phospho-) proteomic experiments, Th-1 cells from each donor were split into three different conditions after initial expansion: Light SILAC media $(40 \mathrm{mg} / \mathrm{ml} \mathrm{L-Lysine} \mathrm{K0} \mathrm{(Sigma,}$ \#L8662) and $84 \mathrm{mg} / \mathrm{ml}$ L-Arginine R0 (Sigma, \#A8094)), medium SILAC media (49 mg/ml LLysine U-13C6 K6 (CKGAS, \#CLM-2247-0.25) and $103 \mathrm{mg} / \mathrm{ml}$ L-Arginine U-13C6 R6 (CKGAS, \#CLM-2265-0.25)) and heavy SILAC media (49.7 mg/ml L-Lysine U-13C6,U-15N2 K8 (CKGAS, \#CNLM-291-H-0.25) and $105.8 \mathrm{mg} / \mathrm{ml}$ L-Arginine U-13C6,U-15N2 R10 (CKGAS, \#CNLM-539-H-0.25)) prepared in RPMI SILAC media (Thermo Scientific, \#88365) supplemented with 10\% dialyzed FBS (HyClone, \#SH30079.03), $5 \mathrm{ml}$ L-Glutamine (Invitrogen, \#25030024), $5 \mathrm{ml}$ Pen/Strep (Invitrogen, \#15140122), $5 \mathrm{ml}$ MEM vitamin solution (Thermo Scientific, \#11120052), $5 \mathrm{ml}$ Selenium-Transferrin-Insulin (Thermo Scientific, \#41400045) and expanded in the presence of $20 \mathrm{ng} / \mathrm{ml} \mathrm{IL-2}$ and $10 \mathrm{ng} / \mathrm{ml}$ anti-IL4 for another 10 days in order to achieve complete labelling. Media was exchanged every two days. Incorporation of medium and heavy version of Lysine and Arginine was checked by mass spectrometry and samples with an incorporation greater than $95 \%$ were used.

After expansion, cells were starved without IL-2 for 24 hours before stimulation with $10 \mathrm{nM} \mathrm{IL-}$ 27 or $20 \mathrm{nM} \mathrm{HylL}-6$ for 15 minutes (phosphoproteomics) or $24 \mathrm{~h}$ (global proteomic changes). 
Cells were then washed three times in ice-cold PBS, mix in a 1:1:1 ratio, resuspended in SDScontaining lysis buffer ( $1 \%$ SDS in $100 \mathrm{mM}$ Triethylammonium Bicarbonate buffer (TEAB)) and incubated on ice for $10 \mathrm{~min}$ to ensure cell lysis. Then, cell lysates were centrifuged at $20000 \mathrm{~g}$ for 10 minutes at $+4^{\circ} \mathrm{C}$ and supernatant was transferred to a clean tube. Protein concentration was determined by using BCA Protein Assay Kit (Thermo, \#23227), and $10 \mathrm{mg}$ of protein per experiment were reduced with 10mM dithiothreitol (DTT, Sigma, \#D0632) for $1 \mathrm{~h}$ at $55^{\circ} \mathrm{C}$ and alkylated with $20 \mathrm{mM}$ iodoacetamide (IAA, Sigma, \#16125) for $30 \mathrm{~min}$ at RT. Protein was then precipitated using six volumes of chilled $\left(-20^{\circ} \mathrm{C}\right)$ acetone overnight. After precipitation, protein pellet was resuspended in $1 \mathrm{ml}$ of $100 \mathrm{mM}$ TEAB and digested with Trypsin (1:100 w/w, Thermo, \#90058) and digested overnight at 37.C. Then, samples were cleared by centrifugation at $20000 \mathrm{~g}$ for $30 \mathrm{~min}$ at $+4^{\circ} \mathrm{C}$, and peptide concentration was quantified with Quantitative Colorimetric Peptide Assay (Thermo, \#23275).

Phosphopeptide enrichment in the peptide fractions generated as described above was carried out using MagResyn Ti-IMAC following manufacturer instructions (2BScientific, MRTIM002).

High $\mathrm{pH}$ reverse phase fractionation for phosphoproteomics:

Samples were dissolved in $200 \mu \mathrm{L}$ of $10 \mathrm{mM}$ ammonium formate buffer $\mathrm{pH} 9.5$ and peptides are fractionated using high $\mathrm{pH}$ RP chromatography. A C18 Column from Waters (XBridge peptide $\mathrm{BEH}, 130 \AA$, $3.5 \mu \mathrm{m} 4.6 \times 150 \mathrm{~mm}$, Ireland) with a guard column (XBridge, C18, 3.5 $\mu \mathrm{m}, 4.6 \times 20 \mathrm{~mm}$, Waters) are used on a Ultimate $3000 \mathrm{HPLC}$ (Thermo-Scientific). Buffers A and $B$ used for fractionation consist, respectively of $10 \mathrm{mM}$ ammonium formate in milliQ water (Buffer $A$ ) and $10 \mathrm{mM}$ ammonium formate in $90 \%$ acetonitrile (Buffer B), both buffers were adjusted to $\mathrm{pH} 9.5$ with ammonia. Fractions are collected using a WPS-3000FC autosampler (Thermo-Scientific) at 1 min intervals. Column and guard column were equilibrated with $2 \%$ buffer $\mathrm{B}$ for $20 \mathrm{~min}$ at a constant flow rate of $0.8 \mathrm{ml} / \mathrm{min}$ and a constant temperature 0 f $21^{\circ} \mathrm{C}$. Samples $(193 \mu \mathrm{l})$ are loaded onto the column at $0.8 \mathrm{ml} / \mathrm{min}$, and separation gradient started from $2 \%$ buffer $B$, to $8 \%$ B in 6 min, then from $8 \%$ B to $45 \%$ B within 54 min and finaly from $45 \% \mathrm{~B}$ to $100 \% \mathrm{~B}$ in $5 \mathrm{~min}$. The column is washed for $15 \mathrm{~min}$ at $100 \%$ buffer $B$ and equilibrated at $2 \%$ buffer $B$ for $20 \mathrm{~min}$ as mentioned above. The fraction collection started 1 min after injection and stopped after $80 \mathrm{~min}$ (total of 80 fractions, $800 \mu \mathrm{l}$ each). Each peptide fraction was acidified immediately after elution from the column by adding 20 to $30 \mu 10 \%$ formic acid to each tube in the autosampler. The total number of fractions concatenated was set to 10 . The content of fractions from each set was dried prior to further analysis.

\section{LC-MS/MS Analysis:}

LC-MS analysis was done at the FingerPrints Proteomics Facility (University of Dundee). Analysis of peptide readout was performed on a $Q$ Exactive ${ }^{\mathrm{TM}}$ plus, Mass Spectrometer (Thermo Scientific) coupled with a Dionex Ultimate 3000 RS (Thermo Scientific). LC buffers used are the following: buffer $A(0.1 \%$ formic acid in Milli-Q water $(\mathrm{v} / \mathrm{v}))$ and buffer $\mathrm{B}(80 \%$ acetonitrile and $0.1 \%$ formic acid in Milli-Q water $(\mathrm{v} / \mathrm{v})$. Dried fractions were resuspended in $35 \mu \mathrm{l}, 1 \%$ formic acid and aliquots of $15 \mu \mathrm{L}$ of each fraction were loaded at $10 \mu \mathrm{L} / \mathrm{min}$ onto a trap column ( $100 \mu \mathrm{m} \times 2 \mathrm{~cm}$, PepMap nanoViper C18 column, $5 \mu \mathrm{m}, 100 \AA$, Thermo Scientific) equilibrated in $0.1 \%$ TFA. The trap column was washed for $5 \mathrm{~min}$ at the same flow rate with $0.1 \%$ TFA and then switched in-line with a Thermo Scientific, resolving C18 column $(75 \mu \mathrm{m} \times$ $50 \mathrm{~cm}$, PepMap RSLC C18 column, $2 \mu \mathrm{m}, 100 \AA$ ). The peptides were eluted from the column 
at a constant flow rate of $300 \mathrm{nl} / \mathrm{min}$ with a linear gradient from $2 \%$ buffer B to $5 \%$ buffer B in 5 min then from $5 \%$ buffer B to $35 \%$ buffer B in $125 \mathrm{~min}$, and finally from $35 \%$ buffer $B$ to $98 \%$ buffer B in $2 \mathrm{~min}$. The column was then washed with $98 \%$ buffer B for $20 \mathrm{~min}$ and reequilibrated in $2 \%$ buffer $B$ for 17 min. The column was kept at a constant temperature of $50^{\circ} \mathrm{C}$. Q-exactive plus was operated in data dependent positive ionization mode. The source voltage was set to $2.5 \mathrm{Kv}$ and the capillary temperature was $250^{\circ} \mathrm{C}$.

A scan cycle comprised MS1 scan (m/z range from 350-1600, ion injection time of $20 \mathrm{~ms}$, resolution 70000 and automatic gain control (AGC) $1 \times 10^{6}$ ) acquired in profile mode, followed by 15 sequential dependent MS2 scans (resolution 17500) of the most intense ions fulfilling predefined selection criteria (AGC $2 \times 10^{5}$, maximum ion injection time $100 \mathrm{~ms}$, isolation window of $1.4 \mathrm{~m} / \mathrm{z}$, fixed first mass of $100 \mathrm{~m} / \mathrm{z}$, spectrum data type: centroid, intensity threshold $2 \times 10^{4}$, exclusion of unassigned, singly and $>7$ charged precursors, peptide match preferred, exclude isotopes on, dynamic exclusion time $45 \mathrm{~s}$ ). The HCD collision energy was set to $27 \%$ of the normalized collision energy. Mass accuracy is checked before the start of samples analysis.

\section{Mass spectrometry data analysis:}

Q Exactive Plus Mass Spectrometer .RAW files were analyzed, and peptides and proteins quantified using MaxQuant (89), using the built-in search engine Andromeda (90). All settings were set as default, except for the minimal peptide length of 5, and Andromeda search engine was configured for the UniProt Homo sapiens protein database (release date: 2018_09). Peptide and protein ratios only quantified in at least two out of the three replicates were considered, and the $p$-values were determined by Student's $t$ test and corrected for multiple testing using the Benjamini-Hochberg procedure (Benjamini and Hochberg, 1995).

\section{Plasmid constructs:}

For single molecule fluorescence microscopy, monomeric non-fluorescent (Y67F) variant of eGFP was N-terminally fused to GP130. This tag (mXFPm) was engineered to specifically bind anti-GFP nanobody "minimizer" ( $\alpha$ GFP-miNB). This construct was inserted into a modified version of pSems-26 m (Covalys) using a signal peptide of Igk. The ORF was linked to a neomycin resistance cassette via an IRES site. A mXFPe-IL-27R $\alpha$ construct was designed likewise but is recognized by $\alpha$ GFP nanobody "enhancer" (mXFPe). The chimeric construct mXFP-IL-27R $\alpha$ (ECD \& TMD)-GP130(ICD) was a fusion construct of IL-27R $\alpha$ (aa 33-540) and GP130 (aa 645-918).

\section{Cell lines and media:}

HeLa cells were grown in DMEM containing $10 \%$ v/v FBS, penicillin-streptomycin, and Lglutamine (2 mM). RPE1 cells were grown in DMEM/F12 containing 10\% v/v FBS, penicillinstreptomycin, and L-glutamine (2 mM). RPE1 cells were stably transfected by mXFPe-IL27R $\alpha$, mutants and the chimeric construct by PEI method according to standard protocols. Using G418 selection $(0.6 \mathrm{mg} / \mathrm{ml})$ individual clones were selected, proliferated and characterized. For comparing receptor cell surface expression levels of stable clones expressing variants of IL-27R $\alpha$, cells were detached using PBS+2mM EDTA, spun down (300g, $5 \mathrm{~min}$ ) and incubated with "enhancer" $\alpha \mathrm{GFP}-\mathrm{enNB}^{\text {Dy647 }}$ (10 nM, $15 \mathrm{~min}$ on ice). After incubation, cells were washed with PBS and run on cytometer. 


\section{Flow cytometry staining and antibodies:}

For measuring dose-response curves of STAT1/3 phosphorylation (either Th-1 cells or RPE1 clones), 96-well plated were prepared with $50 \mu \mathrm{l}$ of cell suspensions at $2 \times 10^{6} \mathrm{cells} / \mathrm{ml} / \mathrm{well}$ for Th- 1 and $2 \times 10^{5}$ cells $/ \mathrm{ml} /$ well for RPE1. The latter were detached using Accutase (Sigma). Cells were stimulated with a set of different concentrations to obtain dose-response curves. To this end cells were stimulated for $15 \mathrm{~min}$ at $37^{\circ} \mathrm{C}$ with the respective cytokines followed by PFA fixation (2\%) for 15 min at RT.

For kinetic experiments, cell suspensions were stimulated with a defined, saturating concentration of cytokines (10 nM IL-27, 20 nM HyplL-6, $100 \mathrm{nM}$ wt-IL-6) in a reverse order so that all cell suspensions were PFA-fixed (2\%) simultaneously. For pSTAT1/3 kinetic experiments at JAK inhibition, Tofacitinib $(2 \mu \mathrm{M}$, Stratech, \#S2789-SEL) was added after 15 min of stimulation and cells were PFA-fixed in correct order.

After fixation (15 min at RT), cells were spun down at $300 \mathrm{~g}$ for $6 \mathrm{~min}$ at $4^{\circ} \mathrm{C}$. Cell pellets were resuspended and permeabilized in ice-cold methanol and kept for $30 \mathrm{~min}$ on ice. After permeabilization cells were fluorescently barcoded according to (91). In brief: using two NHSdyes (PacificBlue, \#10163, DyLight800, \#46421, Thermo Scientific), individual wells were stained with a combination of different concentrations of these dyes. After barcoding, cells are pooled and stained with anti-pSTAT1 ${ }^{\text {Alexa647 }}$ (Cell Signaling Technologies, \#8009) and antipSTAT3 $^{\text {Alexa488 }}$ (Biolegend, \#651006) at a 1:100 dilution in PBS+0.5\%BSA for $1 \mathrm{~h}$ at RT. T-cells were also stained with anti-CD $8^{\text {AlexaFlour700 }}\left(1: 120\right.$, Biolegend, \#300920), anti-CD4 ${ }^{\mathrm{PE}}(1: 120$,

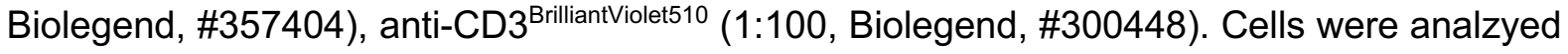
at the flow cytometer (Beckman Coulter, Cytoflex $S$ ) and individual cell populations were identified by their barcoding pattern. Mean fluorescence intensity (MFI) of pSTAT1 ${ }^{647}$ and pSTAT3 ${ }^{488}$ was measured for all individual cell populations.

For measuring total STAT levels, methanol-permeabilized cells were stained with antiSTAT1 ${ }^{\text {Alexa647 }}$ (1:70, Biolegend, \#558560) or anti-STAT3 ${ }^{\text {APC }}$ (1:50, Biolegend, \#560392). Total IRF1 levels methanol-permeabilized cells were stained with anti-IRF1 ${ }^{\text {Alexa647 }}$ (1:50, Biolegend, \#14105). For measuring cell surface levels of GP130, cells were detached with Accutase (Sigma) and stained with anti-GP130 APC (1:100, Biolegend, \#362006) for 1h on ice.

\section{RNA Transcriptome Sequencing:}

Human Th-1 cells from three donors each (StemCell Technologies) were cultivated and stimulated as described in above. Cells were washed in Hank's balanced salt solution (HBSS, Gibco) and snap frozen for storage. RNA was isolated using the RNeasy Kit (Quiagen) according to manufacturer's protocol. All RNA 260/280 ratios were above 1.9. Of each sample, $1 \mu \mathrm{g}$ of RNA was used. Transcriptomic analysis was done by Novogene as follows. Sequencing libraries were generated using NEBNext ${ }^{\circledR}$ UltraTM RNALibrary Prep Kit for Illumina ${ }^{\circledR}$ (NEB, USA) following manufacturer's recommendations and index codes were added to attribute sequences to each sample. Briefly, mRNA was purified from total RNA using poly-T oligo-attached magnetic beads. Fragmentation was carried out using divalent cations under elevated temperature in NEBNext First StrandSynthesis Reaction Buffer (5X). First strand cDNA was synthesized using random hexamer primer and M-MuLV Reverse Transcriptase (RNase $\mathrm{H}$-). Second strand cDNA synthesis was subsequently performed using DNA Polymerase I and RNase $\mathrm{H}$. Remaining overhangs were converted into blunt ends via exonuclease/polymerase activities. After adenylation of 3' ends of DNA fragments, NEBNext Adaptor with hairpin loop structure were ligated to prepare for hybridization. In order to select 
cDNA fragments of preferentially 150 200 bp in length, the library fragments were purified with AMPure XP system (Beckman Coulter, Beverly, USA). Then $3 \mu \mathrm{l}$ USER Enzyme (NEB, USA) was used with size-selected, adaptor-ligated $\mathrm{CDNA}$ at $37^{\circ} \mathrm{C}$ for $15 \mathrm{~min}$ followed by 5 min at $95^{\circ} \mathrm{C}$ before PCR. Then PCR was performed with Phusion High-Fidelity DNA polymerase, Universal PCR primers and Index (X) Primer. At last, PCR products were purified (AMPure XP system) and library quality was assessed on the Agilent Bioanalyzer 2100 system.

\section{RNA Sequencing Data Analysis:}

Primary data analysis for quality control, mapping to reference genome and quantification was conducted by Novogene as outlined below.

Quality control: Raw data (raw reads) of FASTQ format were firstly processed through inhouse scripts. In this step, clean data (clean reads) were obtained by removing reads containing adapter and poly- $\mathrm{N}$ sequences and reads with low quality from raw data. At the same time, Q20, Q30 and GC content of the clean data were calculated. All the downstream analyses were based on the clean data with high quality.

Mapping to reference genome: Reference genome and gene model annotation files were downloaded from genome website browser (NCBI/UCSC/Ensembl) directly. Paired-end clean reads were mapped to the reference genome using HISAT2 software. HISAT2 uses a large set of small GFM indexes that collectively cover the whole genome. These small indexes (called local indexes), combined with several alignment strategies, enable rapid and accurate alignment of sequencing reads.

Quantification: HTSeq was used to count the read numbers mapped of each gene, including known and novel genes. And then RPKM of each gene was calculated based on the length of the gene and reads count mapped to this gene. RPKM, (Reads Per Kilobase of exon model per Million mapped reads), considers the effect of sequencing depth and gene length for the reads count at the same time and is currently the most commonly used method for estimating gene expression levels.

For each identified gene, the fold change was calculated by the ratio of cytokine stimulated/unstimulated expression levels within each donor and an unpaired, two-tailed t test was applied to calculate $p$ values. Genes were considered to be significantly altered if: $p$ value $\leq 0.05$, and $\log _{2}$ fold change $\geq+1$ or $\leq-1$. Genes with an RPKM of less than 1 in two or more donors were excluded from analysis so as to remove genes with abundance near detection limit. Genes without annotated function were also removed. Functional annotation of genes (KEGG pathways, GO terms) was done using DAVID Bioinformatics Resource functional annotation tool $(92,93)$. Clustered heatmap was generated using $\mathrm{R}$ Studio Pheatmap package.

\section{siRNA-mediated knockdown of IRF1 in RPE1 cells:}

A set of four IRF1-siRNAs were purchased from Dharmacon and tested individually to determine levels of knockdown achieved. The siRNA providing the highest level of IRF1. knockdown (Horizon, LQ-011704-00-0005, siRNA \#2: UGAACUCCCUGCCAGAUAU) were subsequently used in all the experiments. RPE1-IL27R $\alpha$ cells were plated in 6-well dishes $\left(0.4 \times 10^{6}\right.$ cells per well) and transfected the next day with IRF1-siRNA or control-GAPDH siRNA (Horizon, D-001830-10-05) (Dharmacon) using DharmaFect 1 transfection reagent (Dharmacon) following the manufacturer's instructions for 24h. At different timepoints of IL-27 (2nM) or HyplL-6 (10nM) stimulation, samples were collected from each one 6-well. Cells were 
trypsinized and each sample was spun down and pellets snap-frozen in liquid nitrogen for subsequent RNA isolation (90\%) or PFA-fixed for total IRF1 staining (10\%) by flow cytometry.

\section{Real-time quantitative PCR:}

Cells were subject to RNA isolation using the Qiagen RNeasy kit. RNA (100 ng) was reverse transcribed to complementary DNA (cDNA) using an iScript cDNA synthesis kit (BioRad, \#1708890), which was used as template for quantitative PCR. PowerTrack ${ }^{\mathrm{TM}}$ SYBR Green Master Mix (Takara, \#A46109) was used for the reaction with the following primers:

\begin{tabular}{|l|l|l|l|}
\hline target & For & Rev & Size \\
\hline$\beta$-actin & CATGTACGTTGCTATCCAGGC & CTCCTTAATGTCACGCACGAT & $250 \mathrm{bp}$ \\
\hline STAT1 & CTAGTGGAGTGGAAGCGGAG & CACCACAAACGAGCTCTGAA & $252 \mathrm{bp}$ \\
\hline GBP5 & TCCTCGGATTATTGCTCGGC & CCTTTGCGCTTCAGCCTTTT & $309 \mathrm{bp}$ \\
\hline OAS1 & GAAGGCAGCTCACGAAACC & AGGCCTCAGCCTCTTGTG & $114 \mathrm{bp}$ \\
\hline SOCS3 & GTCCCCCCAGAAGAGCCTATTA & TTGACGGTCTTCCGACAGAGAT & 118 \\
\hline
\end{tabular}

$\beta$-actin was used as housekeeping gene for normalization. Each siRNA knockdown experiment was performed in three replicates with each sample for qPCR being done in two technical replicates.

\section{Mathematical models and Bayesian inference:}

We developed two new mathematical models, making use of ordinary differential equations (ODEs), for the initial steps of cytokine-receptor binding, dimer formation and signal activation by HypIL-6 and IL-27, respectively; namely, a set of ODEs for the HypIL-6 system and a separate set of ODEs for the IL-27 system (see end of this section for the set of ODEs included in each model). These ODEs describe the rate of change of the concentration for each molecular species considered in the receptor-ligand systems (HyplL-6 and IL-27) over time. By solving these ODEs, a time-course for the concentration of total (free and bound) phosphorylated STAT1 and STAT3 can be obtained and compared to the experimental data (Supp. Fig. 5b \& c). The HypIL-6 and IL-27 mathematical models differ due to the reactions involved in the formation of the signaling dimer for each cytokine. Under stimulation with HypIL-6, two HyplL-6 bound GP130 monomers are required to form the homodimer (Supp. Fig. 3a), whereas under IL-27 stimulation, we assume that IL-27 binds to the IL-27R $\alpha$ chain and not to GP130 (Supp. Fig. 3b) and hence the heterodimer is comprised of an IL-27 molecule bound to an IL-27R $\alpha$ monomer and one GP130 chain. In the mathematical models, we assume that upon formation of the dimers (homo- or heterodimer), these receptor chains become immediately phosphorylated. The models do not consider JAK molecules explicitly. We are assuming that these molecules are constitutively bound to their corresponding receptor chains and that they phosphorylate immediately upon receptor phosphorylation (dimer formation). After the formation of the dimer, which we denote by $D_{6}$ or $D_{27}$, formed by HyplL-6 or IL-27 respectively, the biochemical reactions included in each mathematical model are similar, and are summarized as follows. Table 1 provides a description of the rates for each reaction considered in each (and both) mathematical model(s). In what follows we assume mass action kinetics for all the reactions. A free cytoplasmic unphosphorylated STAT1 or STAT3 molecule can bind to either receptor chain in the dimer, provided that the intracellular tyrosine residue of the receptor in the dimer is free (Supp. Fig. 3c \& d). The STAT1 or STAT3 
molecule can subsequently dissociate from the receptor chain in the dimer or can become phosphorylated (with rate $q$ ) whilst bound to the dimer. We have assumed that the rate of STAT1 or STAT3 phosphorylation when bound does not depend on the STAT type (1 or 3) or on the receptor chain (Supp. Fig. 3c \&d). Phosphorylated STAT1 (pSTAT1) and STAT3 (pSTAT3) molecules can dissociate from the dimer. Once free in the cytoplasm, they can then dephosphorylate (Supp. Fig. 3g). We have assumed that this rate of STAT dephosphorylation only depends on the concentration of the respective PSTAT type, free in the cytoplasm. We note that no allostery has been considered in the models and hence, phosphorylated and unphosphorylated STAT molecules dissociate from the receptor with the same rate (Supp. Fig. 3c \& d). Finally, any molecular species containing receptor molecules can be removed from the system, due to internalisation or degradation, via one of two hypothesised mechanisms (Supp. Fig. 3e \& f):

- hypothesis $1\left(\mathrm{H}_{1}\right)$ : receptors (free or bound, phosphorylated or unphosphorylated) are internalised/degraded with a rate proportional to the concentration of the species in which they are contained, or

- hypothesis $2\left(\mathrm{H}_{2}\right)$ : receptors (free or bound, phosphorylated or unphosphorylated) are internalised/degraded with a rate proportional to the product of the concentration of the species in which they are contained and the sum of the concentrations of free cytoplasmic phosphorylated STAT1 and STAT3.

We note that hypothesis 1 assumes that receptor molecules (free or bound, phosphorylated or unphosphorylated) are being internalised/degraded as part of the natural cellular trafficking cycle. Hypothesis 2 is consistent with a potential feedback mechanism, whereby the free cytoplasmic PSTAT molecules would migrate to the nucleus and increase the production of negative feedback proteins, such as SOCS3, which down-regulate cytokine signaling. Thus, the internalisation/degradation rate of receptor molecules (free or bound, phosphorylated or unphosphorylated) under hypothesis 2 increases with the total amount of free cytoplasmic phosphorylated STAT1 and STAT3, to account for this surface receptor down-regulation. A depiction of the reactions in both the HypIL- 6 and IL-27 mathematical models and under each hypothesis is given in Supp. Fig. 3 where a), c), e) and g) describe the HyplL-6 model and b), d), f) and g) describe the IL-27 model. In this figure, $i \in\{1,3\}$ so that the reactions shown can either involve STAT1 or STAT3. Above or below the reaction arrows is a symbol which represents the rate at which the reaction occurs (under the assumption of mass action kinetics). The notation for the rate constants and initial concentrations in the models, along with their descriptions and units, are given in Table 1.

\begin{tabular}{cll}
\hline Parameter & Description & Unit \\
\hline$r_{1,6}^{+}, r_{1,27}^{+}$ & Rate of receptor-ligand binding & $\mathrm{nM}^{-1} \mathrm{~s}^{-1}$ \\
$r_{1,6}^{-}, r_{1,27}^{-}$ & Rate of receptor-ligand dissociation & $\mathrm{s}^{-1}$ \\
$r_{2,6}^{+}, r_{2,27}^{+}$ & Rate of monomers binding to form a dimer & $\mathrm{nM}^{-1} \mathrm{~s}^{-1}$ \\
$r_{2,6}^{-}, r_{2,27}^{-}$ & Rate of dissociation of the dimer & $\mathrm{s}^{-1}$ \\
$k_{i a}^{+}$ & Rate of STATi binding to GP130 & $\mathrm{nM}^{-1} \mathrm{~s}^{-1}$ \\
$k_{i b}^{+}$ & Rate of STATi binding to IL-27R $\alpha$ & $\mathrm{nM}^{-1} \mathrm{~s}^{-1}$ \\
$k_{i a}^{-}$ & Rate of STATi dissociating GP130 & $\mathrm{s}^{-1}$
\end{tabular}




$\begin{array}{cll}k_{i b}^{-} & \text {Rate of STAT } i \text { dissociating IL-27R } \alpha & \mathrm{s}^{-1} \\ q & \text { Rate of STAT phosphorylation on the dimer } & \mathrm{s}^{-1} \\ d_{i} & \text { Rate of free pSTAT } i \text { dephosphorylation } & \mathrm{s}^{-1} \\ \beta_{6}, \beta_{27} & \text { Rate of receptor internalisation/degradation under hypothesis } 1 & \mathrm{~s}^{-1} \\ \gamma_{6}, \gamma_{27} & \text { Rate of receptor internalisation/degradation under hypothesis } 2 & \mathrm{nM}^{-1} \mathrm{~s}^{-1} \\ {\left[R_{1}(0)\right]} & \text { Initial concentration of GP130 } & \mathrm{nM} \\ {\left[R_{2}(0)\right]} & \text { Initial concentration of IL-27R } & \mathrm{nM} \\ {\left[S_{i}(0)\right]} & \text { Initial concentration of STATi } & \mathrm{nM}\end{array}$

Table 1: Notation, definitions and units for the parameter values used in the mathematical models, where $i \in\{1,3\}$ so that STAT $i$ corresponds to STAT1 or STAT3.

The HyplL-6 mathematical model was formulated based on reactions involving the following species:

- $L_{6}=$ HyplL-6,

- $R_{1}=\mathrm{GP} 130$,

- $C_{1}=$ GP130 - HyplL-6 monomer,

- $D_{6}=$ Phosphorylated GP130 - HyplL-6 - HyplL-6 - GP130 homodimer,

- $S_{1}=$ Unbound cytoplasmic unphosphorylated STAT1,

- $S_{3}=$ Unbound cytoplasmic unphosphorylated STAT3,

- $D_{6} \cdot S_{1}=$ Dimer bound to STAT1,

- $D_{6} \cdot S_{3}=$ Dimer bound to STAT3,

- $D_{6} \cdot p S_{1}=$ Dimer bound to pSTAT1,

- $D_{6} \cdot p S_{3}=$ Dimer bound to pSTAT3,

- $S_{1} \cdot D_{6} \cdot S_{1}=$ Dimer bound to two molecules of STAT1,

- $p S_{1} \cdot D_{6} \cdot S_{1}=$ Dimer bound to two molecules of STAT1, one of which is phosphorylated,

- $p S_{1} \cdot D_{6} \cdot p S_{1}=$ Dimer bound to two molecules of pSTAT1,

- $S_{3} \cdot D_{6} \cdot S_{3}=$ Dimer bound to two molecules of STAT3,

- $p S_{3} \cdot D_{6} \cdot S_{3}=$ Dimer bound to two molecules of STAT3, one of which is phosphorylated,

- $p S_{3} \cdot D_{6} \cdot p S_{3}=$ Dimer bound to two molecules of pSTAT3,

- $S_{1} \cdot D_{6} \cdot S_{3}=$ Dimer bound to one molecule of STAT1 and one of STAT3,

- $p S_{1} \cdot D_{6} \cdot S_{3}=$ Dimer bound to one molecule of pSTAT1 and one of STAT3,

- $S_{1} \cdot D_{6} \cdot p S_{3}=$ Dimer bound to one molecule of STAT1 and one of pSTAT3,

- $p S_{1} \cdot D_{6} \cdot p S_{3}=$ Dimer bound to one molecule of pSTAT1 and one of pSTAT3,

- $p S_{1}=$ Unbound cytoplasmic phosphorylated STAT1,

- $p S_{3}=$ Unbound cytoplasmic phosphorylated STAT3.

The initial reactions in the HyplL-6 signaling pathway can then be described by the ODEs (1) - (22), under the law of mass action, where the terms involving the parameter $\beta_{6}$ apply only to the model under hypothesis 1 and the terms involving the parameter $\gamma_{6}$ apply only to the model under hypothesis 2 . Square brackets around a species is a notation that denotes the concentration of this species with unit nM, and "." implies a reaction bond between two molecules/species. The ODEs are valid for any time $t$, with $t \geq 0$, but time has been omitted in the species concentration for ease of notation. We note here that, for example $\left[R_{1}\right]=\left[R_{1}\right](t)$ for all $t \geq 0$. 


$$
\begin{aligned}
& \frac{d\left[R_{1}\right]}{d t}=-r_{1,6}^{+}\left[R_{1}\right]\left[L_{6}\right]+r_{1,6}^{-}\left[C_{1}\right]-\beta_{6}\left[R_{1}\right]-\gamma_{6}\left(\left[p S_{1}\right]+\left[p S_{3}\right]\right)\left[R_{1}\right] \\
& \frac{d\left[L_{6}\right]}{d t}=-r_{1,6}^{+}\left[R_{1}\right]\left[L_{6}\right]+r_{1,6}^{-}\left[C_{1}\right] \\
& \frac{d\left[C_{1}\right]}{d t}=r_{1,6}^{+}\left[R_{1}\right]\left[L_{6}\right]-r_{1,6}^{-}\left[C_{1}\right]-2 r_{2,6}^{+}\left[C_{1}\right]^{2}+2 r_{2,6}^{-}\left[D_{6}\right]-\beta_{6}\left[C_{1}\right] \\
& -\gamma_{6}\left(\left[p S_{1}\right]+\left[p S_{3}\right]\right)\left[C_{1}\right] \\
& \frac{d\left[D_{6}\right]}{d t}=r_{2,6}^{+}\left[C_{1}\right]^{2}-r_{2,6}^{-}\left[D_{6}\right]-2 k_{1 a}^{+}\left[D_{6}\right]\left[S_{1}\right]+k_{1 a}^{-}\left(\left[D_{6} \cdot S_{1}\right]+\left[D_{6} \cdot p S_{1}\right]\right) \\
& -2 k_{3 a}^{+}\left[D_{6}\right]\left[S_{3}\right]+k_{3 a}^{-}\left(\left[D_{6} \cdot S_{3}\right]+\left[D_{6} \cdot p S_{3}\right]\right)-\beta_{6}\left[D_{6}\right] \\
& -\gamma_{6}\left(\left[p S_{1}\right]+\left[p S_{3}\right]\right)\left[D_{6}\right] \\
& \frac{d\left[S_{1}\right]}{d t}=-k_{1 a}^{+}\left[S_{1}\right]\left(2\left[D_{6}\right]+\left[D_{6} \cdot S_{1}\right]+\left[D_{6} \cdot S_{3}\right]+\left[D_{6} \cdot p S_{1}\right]+\left[D_{6} \cdot p S_{3}\right]\right) \\
& +k_{1 a}^{-}\left(\left[D_{6} \cdot S_{1}\right]+2\left[S_{1} \cdot D_{6} \cdot S_{1}\right]+\left[S_{1} \cdot D_{6} \cdot S_{3}\right]+\left[S_{1} \cdot D_{6} \cdot p S_{1}\right]\right. \\
& \left.+\left[S_{1} \cdot D_{6} \cdot p S_{3}\right]\right)+d_{1}\left[p S_{1}\right] \\
& \frac{d\left[S_{3}\right]}{d t}=-k_{3 a}^{+}\left[S_{3}\right]\left(2\left[D_{6}\right]+\left[D_{6} \cdot S_{3}\right]+\left[D_{6} \cdot S_{1}\right]+\left[D_{6} \cdot p S_{3}\right]+\left[D_{6} \cdot p S_{1}\right]\right) \\
& +k_{3 a}^{-}\left(\left[D_{6} \cdot S_{3}\right]+2\left[S_{3} \cdot D_{6} \cdot S_{3}\right]+\left[S_{1} \cdot D_{6} \cdot S_{3}\right]+\left[S_{3} \cdot D_{6} \cdot p S_{3}\right]\right. \\
& \left.+\left[p S_{1} \cdot D_{6} \cdot S_{3}\right]\right)+d_{3}\left[p S_{3}\right] \\
& \frac{d\left[D_{6} \cdot S_{1}\right]}{d t}=2 k_{1 a}^{+}\left[S_{1}\right]\left[D_{6}\right]-k_{1 a}^{-}\left[D_{6} \cdot S_{1}\right]-k_{1 a}^{+}\left[D_{6} \cdot S_{1}\right]\left[S_{1}\right]+2 k_{1 a}^{-}\left[S_{1} \cdot D_{6} \cdot S_{1}\right] \\
& -k_{3 a}^{+}\left[D_{6} \cdot S_{1}\right]\left[S_{3}\right]+k_{3 a}^{-}\left[S_{1} \cdot D_{6} \cdot S_{3}\right]-q\left[D_{6} \cdot S_{1}\right] \\
& +k_{1 a}^{-}\left[S_{1} \cdot D_{6} \cdot p S_{1}\right]+k_{3 a}^{-}\left[S_{1} \cdot D_{6} \cdot p S_{3}\right]-\beta_{6}\left[D_{6} \cdot S_{1}\right] \\
& -\gamma_{6}\left(\left[p S_{1}\right]+\left[p S_{3}\right]\right)\left[D_{6} \cdot S_{1}\right] \\
& \frac{d\left[D_{6} \cdot S_{3}\right]}{d t}=2 k_{3 a}^{+}\left[S_{3}\right]\left[D_{6}\right]-k_{3 a}^{-}\left[D_{6} \cdot S_{3}\right]-k_{3 a}^{+}\left[D_{6} \cdot S_{3}\right]\left[S_{3}\right]+2 k_{3 a}^{-}\left[S_{3} \cdot D_{6} \cdot S_{3}\right] \\
& -k_{1 a}^{+}\left[D_{6} \cdot S_{3}\right]\left[S_{1}\right]+k_{1 a}^{-}\left[S_{1} \cdot D_{6} \cdot S_{3}\right]-q\left[D_{6} \cdot S_{3}\right]+k_{1 a}^{-}\left[p S_{1} \cdot D_{6} \cdot S_{3}\right] \\
& +k_{3 a}\left[S_{3} \cdot D_{6} \cdot p S_{3}\right]-\beta_{6}\left[D_{6} \cdot S_{3}\right]-\gamma_{6}\left(\left[p S_{1}\right]+\left[p S_{3}\right]\right)\left[D_{6} \cdot S_{3}\right] \\
& \frac{d\left[D_{6} \cdot p S_{1}\right]}{d t}=-k_{1 a}^{+}\left[S_{1}\right]\left[D_{6} \cdot p S_{1}\right]+k_{1 a}^{-}\left[S_{1} \cdot D_{6} \cdot p S_{1}\right]-k_{3 a}^{+}\left[S_{3}\right]\left[D_{6} \cdot p S_{1}\right] \\
& +k_{3 a}^{-}\left[p S_{1} \cdot D_{6} \cdot S_{3}\right]+q\left[D_{6} \cdot S_{1}\right]-k_{1 a}^{-}\left[D_{6} \cdot p S_{1}\right] \\
& +2 k_{1 a}^{-}\left[p S_{1} \cdot D_{6} \cdot p S_{1}\right]+k_{3 a}^{-}\left[p S_{1} \cdot D_{6} \cdot p S_{3}\right]-\beta_{6}\left[D_{6} \cdot p S_{1}\right] \\
& -\gamma_{6}\left(\left[p S_{1}\right]+\left[p S_{3}\right]\right)\left[D_{6} \cdot p S_{1}\right] \\
& \frac{d\left[D_{6} \cdot p S_{3}\right]}{d t}=-k_{3 a}^{+}\left[S_{3}\right]\left[D_{6} \cdot p S_{3}\right]+k_{3 a}^{-}\left[S_{3} \cdot D_{6} \cdot p S_{3}\right]-k_{1 a}^{+}\left[S_{1}\right]\left[D_{6} \cdot p S_{3}\right] \\
& +k_{1 a}^{-}\left[S_{1} \cdot D_{6} \cdot p S_{3}\right]+q\left[D_{6} \cdot S_{3}\right]-k_{3 a}^{-}\left[D_{6} \cdot p S_{3}\right] \\
& +2 k_{3 a}^{-}\left[p S_{3} \cdot D_{6} \cdot p S_{3}\right]+k_{1 a}^{-}\left[p S_{1} \cdot D_{6} \cdot p S_{3}\right]-\beta_{6}\left[D_{6} \cdot p S_{3}\right] \\
& -\gamma_{6}\left(\left[p S_{1}\right]+\left[p S_{3}\right]\right)\left[D_{6} \cdot p S_{3}\right] \\
& \frac{d\left[p S_{1} \cdot D_{6} \cdot S_{1}\right]}{d t}=k_{1 a}^{+}\left[p S_{1} \cdot D_{6}\right]\left[S_{1}\right]-2 k_{1 a}^{-}\left[p S_{1} \cdot D_{6} \cdot S_{1}\right] \\
& +2 q\left[S_{1} \cdot D_{6} \cdot S_{1}\right]-q\left[p S_{1} \cdot D_{6} \cdot S_{1}\right]-\beta_{6}\left[p S_{1} \cdot D_{6} \cdot S_{1}\right]
\end{aligned}
$$




$$
\begin{aligned}
& \frac{d\left[p S_{3} \cdot D_{6} \cdot S_{3}\right]}{d t}-\gamma_{6}\left(\left[p S_{1}\right]+\left[p S_{3}\right]\right)\left[p S_{1} \cdot D_{6} \cdot S_{1}\right] \\
& =k_{3 a}^{+}\left[p S_{3} \cdot D_{6}\right]\left[S_{3}\right]-2 k_{3 a}^{-}\left[p S_{3} \cdot D_{6} \cdot S_{3}\right]+2 q\left[S_{3} \cdot D_{6} \cdot S_{3}\right] \\
& -q\left[p S_{3} \cdot D_{6} \cdot S_{3}\right]-\beta_{6}\left[p S_{3} \cdot D_{6} \cdot S_{3}\right] \\
& -\gamma_{6}\left(\left[p S_{1}\right]+\left[p S_{3}\right]\right)\left[p S_{3} \cdot D_{6} \cdot S_{3}\right] \\
& \frac{d\left[p S_{1} \cdot D_{6} \cdot p S_{1}\right]}{d t}=q\left[p S_{1} \cdot D_{6} \cdot S_{1}\right]-2 k_{1 a}^{-}\left[p S_{1} \cdot D_{6} \cdot p S_{1}\right] \\
& -\beta_{6}\left[p S_{1} \cdot D_{6} \cdot p S_{1}\right]-\gamma_{6}\left(\left[p S_{1}\right]+\left[p S_{3}\right]\right)\left[p S_{1} \cdot D_{6} \cdot p S_{1}\right] \\
& \frac{d\left[p S_{3} \cdot D_{6} \cdot p S_{3}\right]}{d t}=q\left[p S_{3} \cdot D_{6} \cdot S_{3}\right]-2 k_{3 a}^{-}\left[p S_{3} \cdot D_{6} \cdot p S_{3}\right] \\
& -\beta_{6}\left[p S_{3} \cdot D_{6} \cdot p S_{3}\right]-\gamma_{6}\left(\left[p S_{1}\right]+\left[p S_{3}\right]\right)\left[p S_{3} \cdot D_{6} \cdot p S_{3}\right] \\
& \frac{d\left[S_{1} \cdot D_{6} \cdot S_{3}\right]}{d t}=k_{1 a}^{+}\left[S_{1}\right]\left[D_{6} \cdot S_{3}\right]-k_{1 a}^{-}\left[S_{1} \cdot D_{6} \cdot S_{3}\right]+k_{3 a}^{+}\left[S_{1} \cdot D_{6}\right]\left[S_{3}\right] \\
& -k_{3 a}^{-}\left[S_{1} \cdot D_{6} \cdot S_{3}\right]-2 q\left[S_{1} \cdot D_{6} \cdot S_{3}\right]-\beta_{6}\left[S_{1} \cdot D_{6} \cdot S_{3}\right] \\
& -\gamma_{6}\left(\left[p S_{1}\right]+\left[p S_{3}\right]\right)\left[S_{1} \cdot D_{6} \cdot S_{3}\right] \\
& \frac{d\left[p S_{1} \cdot D_{6} \cdot S_{3}\right]}{d t}=q\left[S_{1} \cdot D_{6} \cdot S_{3}\right]+k_{3 a}^{+}\left[p S_{1} \cdot D_{6}\right]\left[S_{3}\right] \\
& -k_{3 a}^{-}\left[p S_{1} \cdot D_{6} \cdot S_{3}\right]-q\left[p S_{1} \cdot D_{6} \cdot S_{3}\right]-k_{1 a}^{-}\left[p S_{1} \cdot D_{6} \cdot S_{3}\right] \\
& -\beta_{6}\left[p S_{1} \cdot D_{6} \cdot S_{3}\right]-\gamma_{6}\left(\left[p S_{1}\right]+\left[p S_{3}\right]\right)\left[p S_{1} \cdot D_{6} \cdot S_{3}\right] \\
& \frac{d\left[S_{1} \cdot D_{6} \cdot p S_{3}\right]}{d t}=q\left[S_{1} \cdot D_{6} \cdot S_{3}\right]+k_{1 a}^{+}\left[S_{1}\right]\left[D_{6} \cdot p S_{3}\right] \\
& -k_{1 a}^{-}\left[S_{1} \cdot D_{6} \cdot p S_{3}\right]-q\left[S_{1} \cdot D_{6} \cdot p S_{3}\right]-k_{3 a}^{-}\left[S_{1} \cdot D_{6} \cdot p S_{3}\right] \\
& -\beta_{6}\left[S_{1} \cdot D_{6} \cdot p S_{3}\right]-\gamma_{6}\left(\left[p S_{1}\right]+\left[p S_{3}\right]\right)\left[S_{1} \cdot D_{6} \cdot p S_{3}\right] \\
& \frac{d\left[p S_{1} \cdot D_{6} \cdot p S_{3}\right]}{d t}=q\left(\left[S_{1} \cdot D_{6} \cdot p S_{3}\right]+\left[p S_{1} \cdot D_{6} \cdot S_{3}\right]\right) \\
& -\left[p S_{1} \cdot D_{6} \cdot p S_{3}\right]\left(k_{1 a}^{-}+k_{3 a}^{-}\right)-\beta_{6}\left[p S_{1} \cdot D_{6} \cdot p S_{3}\right] \\
& -\gamma_{6}\left(\left[p S_{1}\right]+\left[p S_{3}\right]\right)\left[p S_{1} \cdot D_{6} \cdot p S_{3}\right] \\
& \frac{d\left[p S_{1}\right]}{d t}=k_{1 a}^{-}\left(\left[D_{6} \cdot p S_{1}\right]+\left[S_{1} \cdot D_{6} \cdot p S_{1}\right]+\left[S_{3} \cdot D_{6} \cdot p S_{1}\right]+\left[p S_{3} \cdot D_{6} \cdot p S_{1}\right]\right. \\
& \left.+2\left[p S_{1} \cdot D_{6} \cdot p S_{1}\right]\right)-d_{1}\left[p S_{1}\right] \\
& \frac{d\left[p S_{3}\right]}{d t}=k_{3 a}^{-}\left(\left[D_{6} \cdot p S_{3}\right]+\left[S_{3} \cdot D_{6} \cdot p S_{3}\right]+\left[S_{1} \cdot D_{6} \cdot p S_{3}\right]+\left[p S_{1} \cdot D_{6} \cdot p S_{3}\right]\right. \\
& \left.+2\left[p S_{3} \cdot D_{6} \cdot p S_{3}\right]\right)-d_{3}\left[p S_{3}\right]
\end{aligned}
$$

Similarly, and with some species in common with the HyplL-6 model, the IL-27 model has been formulated based on reactions involving the following species:

- $\quad L_{27}=\mathrm{IL}-27$,

- $R_{1}=\mathrm{GP} 130$

- $R_{2}=\mathrm{IL}-27 \mathrm{R} \alpha$,

- $C_{2}=$ IL-27R $\alpha-$ IL-27 monomer,

- $D_{27}=$ Phosphorylated IL-27R $\alpha$ - IL-27 - GP130 heterodimer,

- $S_{1}=$ Unbound cytoplasmic unphosphorylated STAT1,

- $S_{3}=$ Unbound cytoplasmic unphosphorylated STAT3,

- $S_{1} \cdot D_{27}=$ Dimer bound to STAT1 via $R_{1}$, 
- $S_{3} \cdot D_{27}=$ Dimer bound to STAT3 via $R_{1}$,

- $p S_{1} \cdot D_{27}=$ Dimer bound to pSTAT1 via $R_{1}$,

- $p S_{3} \cdot D_{27}=$ Dimer bound to pSTAT3 via $R_{1}$,

- $D_{27} \cdot S_{1}=$ Dimer bound to STAT1 via $R_{2}$,

- $\quad D_{27} \cdot S_{3}=$ Dimer bound to STAT3 via $R_{2}$,

- $D_{27} \cdot p S_{1}=$ Dimer bound to pSTAT1 via $R_{2}$,

- $D_{27} \cdot p S_{3}=$ Dimer bound to pSTAT3 via $R_{2}$,

- $S_{1} \cdot D_{27} \cdot S_{1}=$ Dimer bound to two molecules of STAT1,

- $p S_{1} \cdot D_{27} \cdot S_{1}=$ Dimer bound to two molecules of STAT1, one of them phosphorylated on $R_{1}$,

- $S_{1} \cdot D_{27} \cdot p S_{1}=$ Dimer bound to two molecules of STAT1, one of them phosphorylated on $R_{2}$,

- $p S_{1} \cdot D_{27} \cdot p S_{1}=$ Dimer bound to two molecules of pSTAT1,

- $S_{3} \cdot D_{27} \cdot S_{3}=$ Dimer bound to two molecules of STAT3,

- $p S_{3} \cdot D_{27} \cdot S_{3}=$ Dimer bound to two molecules of STAT3, one of them phosphorylated on $R_{1}$,

- $S_{3} \cdot D_{27} \cdot p S_{3}=$ Dimer bound to two molecules of STAT3, one of them phosphorylated on $R_{2}$,

- $p S_{3} \cdot D_{27} \cdot p S_{3}=$ Dimer bound to two molecules of pSTAT3,

- $S_{1} \cdot D_{27} \cdot S_{3}=$ Dimer bound to STAT1 via $R_{1}$ and STAT3 via $R_{2}$,

- $S_{3} \cdot D_{27} \cdot S_{1}=$ Dimer bound to STAT1 via $R_{2}$ and STAT3 via $R_{1}$,

- $p S_{1} \cdot D_{27} \cdot S_{3}=$ Dimer bound to pSTAT1 via $R_{1}$ and STAT3 via $R_{2}$,

- $S_{3} \cdot D_{27} \cdot p S_{1}=$ Dimer bound to pSTAT1 via $R_{2}$ and STAT3 via $R_{1}$,

- $S_{1} \cdot D_{27} \cdot p S_{3}=$ Dimer bound to STAT1 via $R_{1}$ and pSTAT3 via $R_{2}$,

- $p S_{3} \cdot D_{27} \cdot S_{1}=$ Dimer bound to STAT1 via $R_{2}$ and pSTAT3 via $R_{1}$,

- $p S_{1} \cdot D_{27} \cdot p S_{3}=$ Dimer bound pSTAT1 via $R_{1}$ and pSTAT3 via $R_{2}$,

- $p S_{3} \cdot D_{27} \cdot p S_{1}=$ Dimer bound pSTAT3 via $R_{1}$ and pSTAT1 via $R_{1}$,

- $p S_{1}=$ Unbound cytoplasmic phosphorylated STAT1,

- $p S_{3}=$ Unbound cytoplasmic phosphorylated STAT3.

Again, under the law of mass action, the initial reactions in the IL-27 signaling pathway can be described by the ODEs (23) - (55).

$$
\begin{aligned}
& \frac{d\left[R_{1}\right]}{d t}=-r_{2,27}^{+}\left[C_{2}\right]\left[R_{1}\right]+r_{2,27}^{-}\left[D_{27}\right]-\beta_{27}\left[R_{1}\right]-\gamma_{27}\left(\left[p S_{1}\right]+\left[p S_{3}\right]\right)\left[R_{1}\right] \\
& \frac{d\left[R_{2}\right]}{d t}=-r_{1,27}^{+}\left[R_{2}\right]\left[L_{27}\right]+r_{1,27}^{-}\left[C_{2}\right]-\beta_{27}\left[R_{2}\right]-\gamma_{27}\left(\left[p S_{1}\right]+\left[p S_{3}\right]\right)\left[R_{2}\right] \\
& \frac{d\left[L_{27}\right]}{d t}=-r_{1,27}^{+}\left[R_{2}\right]\left[L_{27}\right]+r_{1,27}^{-}\left[C_{2}\right] \\
& \frac{d\left[C_{2}\right]}{d t}=r_{1,27}^{+}\left[R_{2}\right]\left[L_{27}\right]-r_{1,27}^{-}\left[C_{2}\right]-r_{2,27}^{+}\left[C_{2}\right]\left[R_{1}\right]+r_{2,27}^{-}\left[D_{27}\right]-\beta_{27}\left[C_{2}\right] \\
& -\gamma_{27}\left(\left[p S_{1}\right]+\left[p S_{3}\right]\right)\left[C_{2}\right] \\
& \frac{d\left[D_{27}\right]}{d t}=r_{2,27}^{+}\left[C_{2}\right]\left[R_{1}\right]-r_{2,27}^{-}\left[D_{27}\right]-\left(k_{1 a}^{+}+k_{1 b}^{+}\right)\left[D_{27}\right]\left[S_{1}\right] \\
& +k_{1 a}^{-}\left(\left[S_{1} \cdot D_{27}\right]+\left[p S_{1} \cdot D_{27}\right]\right)+k_{1 b}^{-}\left(\left[D_{27} \cdot S_{1}\right]+\left[D_{27} \cdot p S_{1}\right]\right) \\
& -\left(k_{3 a}^{+}+k_{3 b}^{+}\right)\left[D_{27}\right]\left[S_{3}\right]+k_{3 a}^{-}\left(\left[S_{3} \cdot D_{27}\right]+\left[p S_{3} \cdot D_{27}\right]\right) \\
& +k_{3 b}^{-}\left(\left[D_{27} \cdot S_{3}\right]+\left[D_{27} \cdot p S_{3}\right]\right)-\beta_{27}\left[D_{27}\right] \\
& -\gamma_{27}\left(\left[p S_{1}\right]+\left[p S_{3}\right]\right)\left[D_{27}\right]
\end{aligned}
$$




$$
\begin{aligned}
\frac{d\left[S_{1}\right]}{d t}=-k_{1 a}^{+}[ & \left.S_{1}\right]\left(\left[D_{27}\right]+\left[D_{27} \cdot S_{1}\right]+\left[D_{27} \cdot p S_{1}\right]+\left[D_{27} \cdot S_{3}\right]+\left[D_{27} \cdot p S_{3}\right]\right) \\
& +k_{1 a}^{-}\left(\left[S_{1} \cdot D_{27}\right]+\left[S_{1} \cdot D_{27} \cdot S_{1}\right]+\left[S_{1} \cdot D_{27} \cdot p S_{1}\right]+\left[S_{1} \cdot D_{27} \cdot S_{3}\right]\right. \\
& \left.+\left[S_{1} \cdot D_{27} \cdot p S_{3}\right]\right) \\
& -k_{1 b}^{+}\left[S_{1}\right]\left(\left[D_{27}\right]+\left[S_{1} \cdot D_{27}\right]+\left[p S_{1} \cdot D_{27}\right]+\left[S_{3} \cdot D_{27}\right]\right. \\
& \left.+\left[p S_{3} \cdot D_{27}\right]\right) \\
& +k_{1 b}^{-}\left(\left[D_{27} \cdot S_{1}\right]+\left[S_{1} \cdot D_{27} \cdot S_{1}\right]+\left[p S_{1} \cdot D_{27} \cdot S_{1}\right]+\left[S_{3} \cdot D_{27} \cdot S_{1}\right]\right. \\
& \left.+\left[p S_{3} \cdot D_{27} \cdot S_{1}\right]\right)+d_{1}\left[p S_{1}\right]
\end{aligned}
$$

$$
\begin{aligned}
\frac{d\left[S_{3}\right]}{d t}=-k_{3 a}^{+}[ & \left.S_{3}\right]\left(\left[D_{27}\right]+\left[D_{27} \cdot S_{1}\right]+\left[D_{27} \cdot p S_{1}\right]+\left[D_{27} \cdot S_{3}\right]+\left[D_{27} \cdot p S_{3}\right]\right) \\
& +k_{3 a}^{-}\left(\left[S_{3} \cdot D_{27}\right]+\left[S_{3} \cdot D_{27} \cdot S_{1}\right]+\left[S_{3} \cdot D_{27} \cdot p S_{1}\right]+\left[S_{3} \cdot D_{27} \cdot S_{3}\right]\right. \\
& \left.+\left[S_{3} \cdot D_{27} \cdot p S_{3}\right]\right) \\
& -k_{3 b}^{+}\left[S_{3}\right]\left(\left[D_{27}\right]+\left[S_{1} \cdot D_{27}\right]+\left[p S_{1} \cdot D_{27}\right]+\left[S_{3} \cdot D_{27}\right]\right. \\
& \left.+\left[p S_{3} \cdot D_{27}\right]\right) \\
& +k_{3 b}^{-}\left(\left[D_{27} \cdot S_{3}\right]+\left[S_{1} \cdot D_{27} \cdot S_{3}\right]+\left[p S_{1} \cdot D_{27} \cdot S_{3}\right]+\left[S_{3} \cdot D_{27} \cdot S_{3}\right]\right. \\
& \left.+\left[p S_{3} \cdot D_{27} \cdot S_{3}\right]\right)+d_{3}\left[p S_{3}\right]
\end{aligned}
$$

$$
\begin{aligned}
\frac{d\left[S_{1} \cdot D_{27}\right]}{d t}= & k_{1 a}^{+}\left[S_{1}\right]\left[D_{27}\right]-k_{1 a}^{-}\left[S_{1} \cdot D_{27}\right]-q\left[S_{1} \cdot D_{27}\right]-k_{1 b}^{+}\left[S_{1}\right]\left[S_{1} \cdot D_{27}\right] \\
& +k_{1 b}^{-}\left[S_{1} \cdot D_{27} \cdot S_{1}\right]-k_{3 b}^{+}\left[S_{3}\right]\left[S_{1} \cdot D_{27}\right]+k_{3 b}^{-}\left[S_{1} \cdot D_{27} \cdot S_{3}\right] \\
& +k_{1 b}^{-}\left[S_{1} \cdot D_{27} \cdot p S_{1}\right]+k_{3 b}^{-}\left[S_{1} \cdot D_{27} \cdot p S_{3}\right]-\beta_{27}\left[S_{1} \cdot D_{27}\right] \\
& -\gamma_{27}\left(\left[p S_{1}\right]+\left[p S_{3}\right]\right)\left[S_{1} \cdot D_{27}\right]
\end{aligned}
$$

$$
\begin{aligned}
\frac{d\left[D_{27} \cdot S_{1}\right]}{d t}= & k_{1 b}^{+}\left[S_{1}\right]\left[D_{27}\right]-k_{1 b}^{-}\left[D_{27} \cdot S_{1}\right]-q\left[D_{27} \cdot S_{1}\right]-k_{1 a}^{+}\left[S_{1}\right]\left[D_{27} \cdot S_{1}\right] \\
& +k_{1 a}^{-}\left[S_{1} \cdot D_{27} \cdot S_{1}\right]-k_{3 a}^{+}\left[S_{3}\right]\left[D_{27} \cdot S_{1}\right]+k_{3 a}^{-}\left[S_{3} \cdot D_{27} \cdot S_{1}\right] \\
& +k_{1 a}^{-}\left[p S_{1} \cdot D_{27} \cdot S_{1}\right]+k_{3 a}\left[p S_{3} \cdot D_{27} \cdot S_{1}\right]-\beta_{27}\left[D_{27} \cdot S_{1}\right] \\
& -\gamma_{27}\left(\left[p S_{1}\right]+\left[p S_{3}\right]\right)\left[D_{27} \cdot S_{1}\right]
\end{aligned}
$$$$
\begin{aligned}
\frac{d\left[S_{3} \cdot D_{27}\right]}{d t}= & k_{3 a}^{+}\left[S_{3}\right]\left[D_{27}\right]-k_{3 a}^{-}\left[S_{3} \cdot D_{27}\right]-q\left[S_{3} \cdot D_{27}\right]-k_{3 b}^{+}\left[S_{3}\right]\left[S_{3} \cdot D_{27}\right] \\
& +k_{3 b}^{-}\left[S_{3} \cdot D_{27} \cdot S_{3}\right]-k_{1 b}^{+}\left[S_{1}\right]\left[S_{3} \cdot D_{27}\right]+k_{1 b}^{-}\left[S_{3} \cdot D_{27} \cdot S_{1}\right] \\
& +k_{3 b}^{-}\left[S_{3} \cdot D_{27} \cdot p S_{3}\right]+k_{1 b}^{-}\left[S_{3} \cdot D_{27} \cdot p S_{1}\right]-\beta_{27}\left[S_{3} \cdot D_{27}\right] \\
& -\gamma_{27}\left(\left[p S_{1}\right]+\left[p S_{3}\right]\right)\left[S_{3} \cdot D_{27}\right]
\end{aligned}
$$$$
\frac{d\left[D_{27} \cdot S_{3}\right]}{d t}=k_{3 b}^{+}\left[S_{3}\right]\left[D_{27}\right]-k_{3 b}^{-}\left[D_{27} \cdot S_{3}\right]-q\left[D_{27} \cdot S_{3}\right]-k_{3 a}^{+}\left[S_{3}\right]\left[D_{27} \cdot S_{3}\right]
$$$$
+k_{3 a}^{-}\left[S_{3} \cdot D_{27} \cdot S_{3}\right]-k_{1 a}^{+}\left[S_{1}\right]\left[D_{27} \cdot S_{3}\right]+k_{1 a}^{-}\left[S_{1} \cdot D_{27} \cdot S_{3}\right]
$$$$
+k_{3 a}^{-}\left[p S_{3} \cdot D_{27} \cdot S_{3}\right]+k_{1 a}^{-}\left[p S_{1} \cdot D_{27} \cdot S_{3}\right]-\beta_{27}\left[D_{27} \cdot S_{3}\right]
$$$$
-\gamma_{27}\left(\left[p S_{1}\right]+\left[p S_{3}\right]\right)\left[D_{27} \cdot S_{3}\right]
$$

$$
\begin{aligned}
\frac{d\left[p S_{1} \cdot D_{27}\right]}{d t}= & -k_{1 b}^{+}\left[p S_{1} \cdot D_{27}\right]\left[S_{1}\right]+k_{1 b}^{-}\left[p S_{1} \cdot D_{27} \cdot S_{1}\right]-k_{3 b}^{+}\left[p S_{1} \cdot D_{27}\right]\left[S_{3}\right] \\
& +k_{3 b}^{-}\left[p S_{1} \cdot D_{27} \cdot S_{3}\right]+q\left[S_{1} \cdot D_{27}\right]-k_{1 a}^{-}\left[p S_{1} \cdot D_{27}\right] \\
& +k_{1 b}^{-}\left[p S_{1} \cdot D_{27} \cdot p S_{1}\right]+k_{3 b}^{-}\left[p S_{1} \cdot D_{27} \cdot p S_{3}\right]-\beta_{27}\left[p S_{1} \cdot D_{27}\right] \\
& -\gamma_{27}\left(\left[p S_{1}\right]+\left[p S_{3}\right]\right)\left[p S_{1} \cdot D_{27}\right]
\end{aligned}
$$




$$
\begin{aligned}
\frac{d\left[D_{27} \cdot p S_{1}\right]}{d t}= & -k_{1 a}^{+}\left[D_{27} \cdot p S_{1}\right]\left[S_{1}\right]+k_{1 a}^{-}\left[S_{1} \cdot D_{27} \cdot p S_{1}\right]-k_{3 a}^{+}\left[D_{27} \cdot p S_{1}\right]\left[S_{3}\right] \\
& +k_{3 a}^{-}\left[S_{3} \cdot D_{27} \cdot p S_{1}\right]+q\left[D_{27} \cdot S_{1}\right]-k_{1 b}^{-}\left[D_{27} \cdot p S_{1}\right] \\
& +k_{1 a}^{-}\left[p S_{1} \cdot D_{27} \cdot p S_{1}\right]+k_{3 a}^{-}\left[p S_{3} \cdot D_{27} \cdot p S_{1}\right]-\beta_{27}\left[D_{27} \cdot p S_{1}\right] \\
& -\gamma_{27}\left(\left[p S_{1}\right]+\left[p S_{3}\right]\right)\left[D_{27} \cdot p S_{1}\right]
\end{aligned}
$$

$$
\begin{aligned}
\frac{d\left[p S_{3} \cdot D_{27}\right]}{d t}= & -k_{3 b}^{+}\left[p S_{3} \cdot D_{27}\right]\left[S_{3}\right]+k_{3 b}^{-}\left[p S_{3} \cdot D_{27} \cdot S_{3}\right]-k_{1 b}^{+}\left[p S_{3} \cdot D_{27}\right]\left[S_{1}\right] \\
& +k_{1 b}^{-}\left[p S_{3} \cdot D_{27} \cdot S_{1}\right]+q\left[S_{3} \cdot D_{27}\right]-k_{3 a}^{-}\left[p S_{3} \cdot D_{27}\right] \\
& +k_{3 b}^{-}\left[p S_{3} \cdot D_{27} \cdot p S_{3}\right]+k_{1 b}^{-}\left[p S_{3} \cdot D_{27} \cdot p S_{1}\right]-\beta_{27}\left[p S_{3} \cdot D_{27}\right] \\
& -\gamma_{27}\left(\left[p S_{1}\right]+\left[p S_{3}\right]\right)\left[p S_{3} \cdot D_{27}\right]
\end{aligned}
$$

$$
\begin{aligned}
\frac{d\left[D_{27} \cdot p S_{3}\right]}{d t}= & -k_{3 a}^{+}\left[D_{27} \cdot p S_{3}\right]\left[S_{3}\right]+k_{3 a}^{-}\left[S_{3} \cdot D_{27} \cdot p S_{3}\right]-k_{1 a}^{+}\left[D_{27} \cdot p S_{3}\right]\left[S_{1}\right] \\
& +k_{1 a}^{-}\left[S_{1} \cdot D_{27} \cdot p S_{3}\right]+q\left[D_{27} \cdot S_{3}\right]-k_{3 b}^{-}\left[D_{27} \cdot p S_{3}\right] \\
& +k_{3 a}^{-}\left[p S_{3} \cdot D_{27} \cdot p S_{3}\right]+k_{1 a}^{-}\left[p S_{1} \cdot D_{27} \cdot p S_{3}\right]-\beta_{27}\left[D_{27} \cdot p S_{3}\right] \\
& -\gamma_{27}\left(\left[p S_{1}\right]+\left[p S_{3}\right]\right)\left[D_{27} \cdot p S_{3}\right]
\end{aligned}
$$

$$
\begin{aligned}
\frac{d\left[S_{1} \cdot D_{27} \cdot S_{1}\right]}{d t} & =k_{1 a}^{+}\left[S_{1}\right]\left[D_{27} \cdot S_{1}\right]-k_{1 a}^{-}\left[S_{1} \cdot D_{27} \cdot S_{1}\right] \\
& +k_{1 b}^{+}\left[S_{1} \cdot D_{27}\right]\left[S_{1}\right]-k_{1 b}^{-}\left[S_{1} \cdot D_{27} \cdot S_{1}\right]-2 q\left[S_{1} \cdot D_{27} \cdot S_{1}\right] \\
& -\beta_{27}\left[S_{1} \cdot D_{27} \cdot S_{1}\right]-\gamma_{27}\left(\left[p S_{1}\right]+\left[p S_{3}\right]\right)\left[S_{1} \cdot D_{27} \cdot S_{1}\right]
\end{aligned}
$$

$$
\begin{aligned}
\frac{d\left[p S_{1} \cdot D_{27} \cdot S_{1}\right]}{d t} & =k_{1 b}^{+}\left[p S_{1} \cdot D_{27}\right]\left[S_{1}\right]-k_{1 b}^{-}\left[p S_{1} \cdot D_{27} \cdot S_{1}\right] \\
& +q\left[S_{1} \cdot D_{27} \cdot S_{1}\right]-q\left[p S_{1} \cdot D_{27} \cdot S_{1}\right]-k_{1 a}^{-}\left[p S_{1} \cdot D_{27} \cdot S_{1}\right] \\
& -\beta_{27}\left[p S_{1} \cdot D_{27} \cdot S_{1}\right]-\gamma_{27}\left(\left[p S_{1}\right]+\left[p S_{3}\right]\right)\left[p S_{1} \cdot D_{27} \cdot S_{1}\right]
\end{aligned}
$$

$$
\begin{aligned}
\frac{d\left[S_{1} \cdot D_{27} \cdot p S_{1}\right]}{d t} & =k_{1 a}^{+}\left[S_{1}\right]\left[D_{27} \cdot p S_{1}\right]-k_{1 a}^{-}\left[S_{1} \cdot D_{27} \cdot p S_{1}\right] \\
& +q\left[S_{1} \cdot D_{27} \cdot S_{1}\right]-q\left[S_{1} \cdot D_{27} \cdot p S_{1}\right]-k_{1 b}^{-}\left[S_{1} \cdot D_{27} \cdot p S_{1}\right] \\
& -\beta_{27}\left[S_{1} \cdot D_{27} \cdot p S_{1}\right]-\gamma_{27}\left(\left[p S_{1}\right]+\left[p S_{3}\right]\right)\left[S_{1} \cdot D_{27} \cdot p S_{1}\right]
\end{aligned}
$$

$$
\begin{aligned}
\frac{d\left[p S_{1} \cdot D_{27} \cdot p S_{1}\right]}{d t} & =q\left(\left[S_{1} \cdot D_{27} \cdot p S_{1}\right]+\left[p S_{1} \cdot D_{27} \cdot S_{1}\right]\right) \\
& -\left[p S_{1} \cdot D_{27} \cdot p S_{1}\right]\left(k_{1 a}^{-}+k_{1 b}^{-}\right)-\beta_{27}\left[p S_{1} \cdot D_{27} \cdot p S_{1}\right] \\
& -\gamma_{27}\left(\left[p S_{1}\right]+\left[p S_{3}\right]\right)\left[p S_{1} \cdot D_{27} \cdot p S_{1}\right]
\end{aligned}
$$

$$
\begin{aligned}
\frac{d\left[S_{3} \cdot D_{27} \cdot S_{3}\right]}{d t} & =k_{3 a}^{+}\left[S_{3}\right]\left[D_{27} \cdot S_{3}\right]-k_{3 a}^{-}\left[S_{3} \cdot D_{27} \cdot S_{3}\right] \\
& +k_{3 b}^{+}\left[S_{3} \cdot D_{27}\right]\left[S_{3}\right]-k_{3 b}^{-}\left[S_{3} \cdot D_{27} \cdot S_{3}\right]-2 q\left[S_{3} \cdot D_{27} \cdot S_{3}\right] \\
& -\beta_{27}\left[S_{3} \cdot D_{27} \cdot S_{3}\right]-\gamma_{27}\left(\left[p S_{1}\right]+\left[p S_{3}\right]\right)\left[S_{3} \cdot D_{27} \cdot S_{3}\right]
\end{aligned}
$$

$$
\begin{aligned}
\frac{d\left[p S_{3} \cdot D_{27} \cdot S_{3}\right]}{d t} & =k_{3 b}^{+}\left[p S_{3} \cdot D_{27}\right]\left[S_{3}\right]-k_{3 b}^{-}\left[p S_{3} \cdot D_{27} \cdot S_{3}\right] \\
& +q\left[S_{3} \cdot D_{27} \cdot S_{3}\right]-q\left[p S_{3} \cdot D_{27} \cdot S_{3}\right]-k_{3 a}^{-}\left[p S_{3} \cdot D_{27} \cdot S_{3}\right] \\
& -\beta_{27}\left[p S_{3} \cdot D_{27} \cdot S_{3}\right]-\gamma_{27}\left(\left[p S_{1}\right]+\left[p S_{3}\right]\right)\left[p S_{3} \cdot D_{27} \cdot S_{3}\right]
\end{aligned}
$$$$
\frac{d\left[S_{3} \cdot D_{27} \cdot p S_{3}\right]}{d t}=k_{3 a}^{+}\left[S_{3}\right]\left[D_{27} \cdot p S_{3}\right]-k_{3 a}^{-}\left[S_{3} \cdot D_{27} \cdot p S_{3}\right]
$$ 


$$
\begin{aligned}
& +q\left[S_{3} \cdot D_{27} \cdot S_{3}\right]-q\left[S_{3} \cdot D_{27} \cdot p S_{3}\right]-k_{3 b}^{-}\left[S_{3} \cdot D_{27} \cdot p S_{3}\right] \\
& -\beta_{27}\left[S_{3} \cdot D_{27} \cdot p S_{3}\right]-\gamma_{27}\left(\left[p S_{1}\right]+\left[p S_{3}\right]\right)\left[S_{3} \cdot D_{27} \cdot p S_{3}\right]
\end{aligned}
$$

$$
\begin{aligned}
\frac{d\left[p S_{3} \cdot D_{27} \cdot p S_{3}\right]}{d t} & =q\left(\left[S_{3} \cdot D_{27} \cdot p S_{3}\right]+\left[p S_{3} \cdot D_{27} \cdot S_{3}\right]\right) \\
& -\left[p S_{3} \cdot D_{27} \cdot p S_{3}\right]\left(k_{3 a}^{-}+k_{3 b}^{-}\right)-\beta_{27}\left[p S_{3} \cdot D_{27} \cdot p S_{3}\right] \\
& -\gamma_{27}\left(\left[p S_{1}\right]+\left[p S_{3}\right]\right)\left[p S_{3} \cdot D_{27} \cdot p S_{3}\right]
\end{aligned}
$$

$$
\begin{aligned}
\frac{d\left[S_{1} \cdot D_{27} \cdot S_{3}\right]}{d t} & =k_{1 a}^{+}\left[S_{1}\right]\left[D_{27} \cdot S_{3}\right]-k_{1 a}^{-}\left[S_{1} \cdot D_{27} \cdot S_{3}\right] \\
& +k_{3 b}^{+}\left[S_{1} \cdot D_{27}\right]\left[S_{3}\right]-k_{3 b}^{-}\left[S_{1} \cdot D_{27} \cdot S_{3}\right]-2 q\left[S_{1} \cdot D_{27} \cdot S_{3}\right] \\
& -\beta_{27}\left[S_{1} \cdot D_{27} \cdot S_{3}\right]-\gamma_{27}\left(\left[p S_{1}\right]+\left[p S_{3}\right]\right)\left[S_{1} \cdot D_{27} \cdot S_{3}\right]
\end{aligned}
$$

$$
\begin{aligned}
\frac{d\left[S_{3} \cdot D_{27} \cdot S_{1}\right]}{d t} & =k_{3 a}^{+}\left[S_{3}\right]\left[D_{27} \cdot S_{1}\right]-k_{3 a}^{-}\left[S_{3} \cdot D_{27} \cdot S_{1}\right] \\
& +k_{1 b}^{+}\left[S_{3} \cdot D_{27}\right]\left[S_{1}\right]-k_{1 b}^{-}\left[S_{3} \cdot D_{27} \cdot S_{1}\right]-2 q\left[S_{3} \cdot D_{27} \cdot S_{1}\right] \\
& -\beta_{27}\left[S_{3} \cdot D_{27} \cdot S_{1}\right]-\gamma_{27}\left(\left[p S_{1}\right]+\left[p S_{3}\right]\right)\left[S_{3} \cdot D_{27} \cdot S_{1}\right]
\end{aligned}
$$

$$
\begin{aligned}
\frac{d\left[p S_{1} \cdot D_{27} \cdot S_{3}\right]}{d t} & =k_{3 b}^{+}\left[p S_{1} \cdot D_{27}\right]\left[S_{3}\right]-k_{3 b}^{-}\left[p S_{1} \cdot D_{27} \cdot S_{3}\right] \\
& +q\left[S_{1} \cdot D_{27} \cdot S_{3}\right]-q\left[p S_{1} \cdot D_{27} \cdot S_{3}\right]-k_{1 a}^{-}\left[p S_{1} \cdot D_{27} \cdot S_{3}\right] \\
& -\beta_{27}\left[p S_{1} \cdot D_{27} \cdot S_{3}\right]-\gamma_{27}\left(\left[p S_{1}\right]+\left[p S_{3}\right]\right)\left[p S_{1} \cdot D_{27} \cdot S_{3}\right]
\end{aligned}
$$

$$
\begin{aligned}
\frac{d\left[p S_{3} \cdot D_{27} \cdot S_{1}\right]}{d t} & =k_{1 b}^{+}\left[p S_{3} \cdot D_{27}\right]\left[S_{1}\right]-k_{1 b}^{-}\left[p S_{3} \cdot D_{27} \cdot S_{1}\right] \\
& +q\left[S_{3} \cdot D_{27} \cdot S_{1}\right]-q\left[p S_{3} \cdot D_{27} \cdot S_{1}\right]-k_{3 a}^{-}\left[p S_{3} \cdot D_{27} \cdot S_{1}\right] \\
& -\beta_{27}\left[p S_{3} \cdot D_{27} \cdot S_{1}\right]-\gamma_{27}\left(\left[p S_{1}\right]+\left[p S_{3}\right]\right)\left[p S_{3} \cdot D_{27} \cdot S_{1}\right]
\end{aligned}
$$

$$
\begin{aligned}
\frac{d\left[S_{1} \cdot D_{27} \cdot p S_{3}\right]}{d t} & =k_{1 a}^{+}\left[S_{1}\right]\left[D_{27} \cdot p S_{3}\right]-k_{1 a}^{-}\left[S_{1} \cdot D_{27} \cdot p S_{3}\right] \\
& +q\left[S_{1} \cdot D_{27} \cdot S_{3}\right]-q\left[S_{1} \cdot D_{27} \cdot p S_{3}\right]-k_{3 b}^{-}\left[S_{1} \cdot D_{27} \cdot p S_{3}\right] \\
& -\beta_{27}\left[S_{1} \cdot D_{27} \cdot p S_{3}\right]-\gamma_{27}\left(\left[p S_{1}\right]+\left[p S_{3}\right]\right)\left[S_{1} \cdot D_{27} \cdot p S_{3}\right]
\end{aligned}
$$

$$
\begin{aligned}
\frac{d\left[S_{3} \cdot D_{27} \cdot p S_{1}\right]}{d t} & =k_{3 a}^{+}\left[S_{3}\right]\left[D_{27} \cdot p S_{1}\right]-k_{3 a}^{-}\left[S_{3} \cdot D_{27} \cdot p S_{1}\right] \\
& +q\left[S_{3} \cdot D_{27} \cdot S_{1}\right]-q\left[S_{3} \cdot D_{27} \cdot p S_{1}\right]-k_{1 b}^{-}\left[S_{3} \cdot D_{27} \cdot p S_{1}\right] \\
& -\beta_{27}\left[S_{3} \cdot D_{27} \cdot p S_{1}\right]-\gamma_{27}\left(\left[p S_{1}\right]+\left[p S_{3}\right]\right)\left[S_{3} \cdot D_{27} \cdot p S_{1}\right]
\end{aligned}
$$

$$
\begin{aligned}
\frac{d\left[p S_{1} \cdot D_{27} \cdot p S_{3}\right]}{d t} & =q\left(\left[S_{1} \cdot D_{27} \cdot p S_{3}\right]+\left[p S_{1} \cdot D_{27} \cdot S_{3}\right]\right) \\
& -\left[p S_{1} \cdot D_{27} \cdot p S_{3}\right]\left(k_{1 a}^{-}+k_{3 b}^{-}\right)-\beta_{27}\left[p S_{1} \cdot D_{27} \cdot p S_{3}\right] \\
& -\gamma_{27}\left(\left[p S_{1}\right]+\left[p S_{3}\right]\right)\left[p S_{1} \cdot D_{27} \cdot p S_{3}\right]
\end{aligned}
$$

$$
\begin{aligned}
\frac{d\left[p S_{3} \cdot D_{27} \cdot p S_{1}\right]}{d t} & =q\left(\left[S_{3} \cdot D_{27} \cdot p S_{1}\right]+\left[p S_{3} \cdot D_{27} \cdot S_{1}\right]\right) \\
& -\left[p S_{3} \cdot D_{27} \cdot p S_{1}\right]\left(k_{3 a}^{-}+k_{1 b}^{-}\right)-\beta_{27}\left[p S_{3} \cdot D_{27} \cdot p S_{1}\right] \\
& -\gamma_{27}\left(\left[p S_{1}\right]+\left[p S_{3}\right]\right)\left[p S_{3} \cdot D_{27} \cdot p S_{1}\right]
\end{aligned}
$$




$$
\begin{aligned}
& \frac{d\left[p S_{1}\right]}{d t}=k_{1 a}^{-}\left(\left[p S_{1} \cdot D_{27}\right]+\left[p S_{1} \cdot D_{27} \cdot S_{1}\right]+\left[p S_{1} \cdot D_{27} \cdot p S_{1}\right]+\left[p S_{1} \cdot D_{27} \cdot S_{3}\right]\right. \\
& \left.+\left[p S_{1} \cdot D_{27} \cdot p S_{3}\right]\right) \\
& +k_{1 b}^{-}\left(\left[D_{27} \cdot p S_{1}\right]+\left[S_{1} \cdot D_{27} \cdot p S_{1}\right]+\left[p S_{1} \cdot D_{27} \cdot p S_{1}\right]\right. \\
& \left.+\left[S_{3} \cdot D_{27} \cdot p S_{1}\right]+\left[p S_{3} \cdot D_{27} \cdot p S_{1}\right]\right)-d_{1}\left[p S_{1}\right] \\
& \frac{d\left[p S_{3}\right]}{d t}=k_{3 a}^{-}\left(\left[p S_{3} \cdot D_{27}\right]+\left[p S_{3} \cdot D_{27} \cdot S_{3}\right]+\left[p S_{3} \cdot D_{27} \cdot p S_{3}\right]+\left[p S_{3} \cdot D_{27} \cdot S_{1}\right]\right. \\
& \left.+\left[p S_{3} \cdot D_{27} \cdot p S_{1}\right]\right) \\
& +k_{3 b}^{-}\left(\left[D_{27} \cdot p S_{3}\right]+\left[S_{3} \cdot D_{27} \cdot p S_{3}\right]+\left[p S_{3} \cdot D_{27} \cdot p S_{3}\right]\right. \\
& \left.+\left[S_{1} \cdot D_{27} \cdot p S_{3}\right]+\left[p S_{1} \cdot D_{27} \cdot p S_{3}\right]\right)-d_{3}\left[p S_{3}\right]
\end{aligned}
$$

Similarly to the HyplL-6 model, the terms in Equations (23) - (55) involving the parameter $\beta_{27}$ apply only to the model under hypothesis 1 and the terms involving the parameter $\gamma_{27}$ apply only to the model under hypothesis 2 .

We now describe how we have made use of the experimental data (Fig. $6 \mathrm{~b}$ and $6 \mathrm{c}$ supp.) to parameterise the mathematical models described above. Since the experimental outputs are levels of pSTAT1 and pSTAT3 as a function of time under HypIL-6 and IL-27 stimulation (Fig. $6 b$ and $6 c$ supp.), we consider two model outputs of interest for the HyplL-6 and IL-27 mathematical models, which are proportional to the experimental data in Supp. Figure $6 \mathrm{~b}$ and $6 \mathrm{c}$; namely, the sum of all molecular species (variables) containing phosphorylated STAT1 (free or bound) $\left(\left[p S_{1}\right]^{T, j}\right.$, for $\left.j \in\{6,27\}\right)$ and the sum of all species (variables) containing phosphorylated STAT3 (free or bound) $\left(\left[p S_{3}\right]^{T, j}\right.$, for $\left.j \in\{6,27\}\right)$. The concentrations of the two model outputs of interest at any time $t$ are given by

$$
\begin{aligned}
{\left[p S_{1}\right]^{T, 6}(t) } & =\left[D_{6} \cdot p S_{1}\right](t)+\left[p S_{1} \cdot D_{6} \cdot S_{1}\right](t)+2\left[p S_{1} \cdot D_{6} \cdot p S_{1}\right](t)+\left[p S_{1} \cdot D_{6} \cdot S_{3}\right](t) \\
& +\left[p S_{1} \cdot D_{6} \cdot p S_{3}\right](t)+\left[p S_{1}\right](t) \\
{\left[p S_{3}\right]^{T, 6}(t) } & =\left[D_{6} \cdot p S_{3}\right](t)+\left[p S_{3} \cdot D_{6} \cdot S_{3}\right](t)+2\left[p S_{3} \cdot D_{6} \cdot p S_{3}\right](t)+\left[p S_{3} \cdot D_{6} \cdot S_{1}\right](t) \\
& +\left[p S_{3} \cdot D_{6} \cdot p S_{1}\right](t)+\left[p S_{3}\right](t)
\end{aligned}
$$

for the HyplL-6 model, and by

$$
\begin{aligned}
{\left[p S_{1}\right]^{T, 27}(t) } & =\left[p S_{1} \cdot D_{27}\right](t)+\left[D_{27} \cdot p S_{1}\right](t)+\left[p S_{1} \cdot D_{27} \cdot S_{1}\right](t)+\left[S_{1} \cdot D_{27} \cdot p S_{1}\right](t) \\
& +2\left[p S_{1} \cdot D_{27} \cdot p S_{1}\right](t)+\left[p S_{1} \cdot D_{27} \cdot S_{3}\right](t)+\left[S_{3} \cdot D_{27} \cdot p S_{1}\right](t) \\
& +\left[p S_{1} \cdot D_{6} \cdot p S_{3}\right](t)+\left[p S_{3} \cdot D_{6} \cdot p S_{1}\right](t)+\left[p S_{1}\right](t) \\
{\left[p S_{3}\right]^{T, 27}(t) } & =\left[p S_{3} \cdot D_{27}\right](t)+\left[D_{27} \cdot p S_{3}\right](t)+\left[p S_{3} \cdot D_{27} \cdot S_{3}\right](t)+\left[S_{3} \cdot D_{27} \cdot p S_{3}\right](t) \\
& +2\left[p S_{3} \cdot D_{27} \cdot p S_{3}\right](t)+\left[p S_{3} \cdot D_{27} \cdot S_{1}\right](t)+\left[S_{1} \cdot D_{27} \cdot p S_{3}\right](t) \\
& +\left[p S_{1} \cdot D_{6} \cdot p S_{3}\right](t)+\left[p S_{3} \cdot D_{6} \cdot p S_{1}\right](t)+\left[p S_{3}\right](t),
\end{aligned}
$$

for the IL-27 model.

Having developed two mathematical models for the stimulation of the experimental system with HypIL-6 and IL-27, it was then our objective to parameterise these models making use of approximate Bayesian computation sequential Monte Carlo (ABC-SMC). Firstly, a Bayesian model selection was carried out to determine which hypothesis (mechanism) of internalisation/degradation of receptor molecules is most likely given the data. Once a hypothesis was selected, together with the experimental data, the ABC-SMC method allows one to obtain posterior distributions for each of the parameter values and initial concentrations in the mathematical models. In this way, we can learn about which reactions and parameters in the models are causing the differential signaling by PSTAT1 observed when stimulating with HypIL-6 and IL-27. The experimental data we used to compare with the mathematical model 
outputs, was the mean relative fluorescence intensity of total phosphorylated STAT1 and total phosphorylated STAT3 in both RPE1 and Th-1 cells (Supp. Figure 5b and 5c). We normalised the data to obtain dimensionless values, which can be compared with the mathematical model outputs. Firstly, we constructed a linear model for the fluorescence intensity (background fluorescence) of antibodies for phosphorylated STAT1 and STAT3 in unstimulated cells. We subtracted the value of this linear model at each time point from the corresponding fluorescence intensity in HyplL-6 and IL-27 stimulated cells, for each repeat of the experiment and each cell type. Denoting by $f$ the experimental fluorescence intensity, $f(r, i, t p, j, d)$ corresponds to the fluorescence intensity for the $r$ th repeat, $r \in R=\{1,2,3,4\}$ with antibody for STAT $i, i \in I=\{1,3\}$ at time point

$t p \in T P=\{0 \mathrm{~min}, 5 \mathrm{~min}, 15 \mathrm{~min}, 30 \mathrm{~min}, 60 \mathrm{~min}, 90 \mathrm{~min}, 120 \mathrm{~min}, 180 \mathrm{~min}\}$ under stimulation by cytokine IL- $j$ (HyplL- $j$ when $j=6$ ), with $j \in J=\{6,27\}$ and in cell type $d \in$ $D=\{$ RPE1,Th-1 $\}$. Each data point data $(r, i, t p, j, d)$, to be used in the Bayesian inference and Bayesian model selection was then computed as

$$
\operatorname{data}(r, i, t p, j, d)=\frac{f(r, i, t p, j, d)}{f(r, i, t p=30 \mathrm{~min}, j=27, d)}
$$

To compare the model output, sim, with the data, the output was normalised in the same way as the data, i.e.,

$$
\operatorname{sim}(i, t p, j, d)=\frac{\left[p S_{i}\right]^{T, j}(t p, d)}{\left[p S_{i}\right]^{T, 27}(30 \min , d)}
$$

where $\left[p S_{i}\right]^{T, j}(t p, d)$ denotes the total concentration of phosphorylated STAT $i$ at time $t p$ (see Equations 56-59) when considering cell type $d$. In this way, experimental data and the mathematical model outputs are comparable.

The similarity between the model output and the data points is then computed by the introduction of a distance measure $\delta(\mathrm{sim}, \mathrm{data})$. Here, this distance measure was chosen as a generalisation of the Euclidean distance, where

$$
\delta^{d}(\operatorname{sim}, d a t a)^{2}=\sum_{i \in I} \sum_{t p \in T P} \sum_{j \in J}\left(\operatorname{sim}(i, t p, j, d)-\mu_{\text {data }}(i, t p, j, d)\right)^{2},
$$

for $d \in D=\{\operatorname{RPE} 1, \mathrm{Th}-1\}$, where $\mu_{\text {data }}(i, t p, j, d)$ is the mean of the four repeats of the data and is given by

$$
\mu_{d a t a}(i, t p, j, d)=\frac{1}{4} \sum_{r=1}^{4} \operatorname{data}(r, i, t p, j, d) .
$$

To carry out the Bayesian model selection and Bayesian parameter inference, prior beliefs about the parameters were firstly defined. Each of the parameters (reaction rates) and initial concentrations in the model were sampled from a prior distribution, where the distribution was informed by experimental data or values from the literature, when possible. The choice of prior distributions is given in Table 2.

\begin{tabular}{lll}
\hline Parameter & Prior distribution & Reference \\
\hline$r_{1,6}^{+}$ & $10^{r}$ for $r \sim N(-3,1.5)$ & $*$ \\
$r_{1,6}^{-}$ & $10^{r}$ for $r \sim N(-3.9,1.96)$ & $*$ \\
$r_{1,27}^{+}$ & $10^{r}$ for $r \sim N(-2.34,1.17)$ & $*$
\end{tabular}




$$
\begin{aligned}
& r_{1,27}^{-} \\
& r_{2, i}^{+} \text {for } j \in\{6,27\} \\
& r_{2, i}^{-} \text {for } j \in\{6,27\} \\
& k_{i a}^{+}, k_{i b}^{+} \text {for } i \in\{1,3\} \\
& k_{i a}^{-}, k_{i b}^{-} \text {for } i \in\{1,3\} \\
& q \\
& d_{i} \text { for } i \in\{1,3\} \\
& \beta_{j} \text { for } j \in\{6,27\} \\
& {\left[R_{1}(0)\right]} \\
& {\left[R_{2}(0)\right]} \\
& {\left[S_{1}(0)\right]} \\
& {\left[S_{3}(0)\right]}
\end{aligned}
$$$$
10^{r} \text { for } r \sim N(-2.82,1.41)
$$$$
10^{r} \text { for } r \sim \operatorname{Unif}(-2,3)
$$$$
10^{r} \text { for } r \sim \operatorname{Unif}(-3,1)
$$$$
10^{r} \text { for } r \sim \operatorname{Unif}(-7,1)
$$$$
10^{r} \text { for } r \sim \operatorname{Unif}(-2,1)
$$$$
10^{r} \text { for } r \sim \operatorname{Unif}(-3,2)
$$$$
10^{r} \text { for } r \sim \operatorname{Unif}(-5,-2)
$$$$
10^{r} \text { for } r \sim \operatorname{Unif}(-5,-1)
$$$$
N(12.7,6.35)
$$$$
N(33.8,16.9)
$$$$
N(300,100)
$$$$
N(400,100)
$$

Table 2: Prior distributions assigned to each parameter and initial concentration in the model. * These distributions are centred around measurements obtained from cell surface receptor quantification experiments. ${ }^{* *}$ These distributions were derived based on $K_{d}$ values obtained from the literature (42). ${ }^{* * *}$ These distributions are based on values derived from experimental data in which the cells were treated with Tofacitinib. $\dagger$ These distributions were based on values derived from experimental data in which the cells were treated with cycloheximide. $\ddagger$ These distributions were based on computations involving approximate cell sizes and average numbers of molecules per cell.

We made use of the prior distributions from Table 2 to then carry out a Bayesian model selection to determine which hypothesis is most likely given the RPE1 data for both HyplL-6 and IL-27 signaling. We ran $10^{6}$ simulations for each mathematical model (HyplL-6 and IL-27) and for each hypothesis, sampling model parameters from their prior distributions. We then computed a summary statistic for varying values of $\delta^{R P E 1, *}$, the distance threshold between the mathematical model and data at which parameters are accepted (or rejected) in the ABC. Finally, we computed $f\left(H_{K}\right)$, the number of accepted parameter sets for hypothesis $k$, where the parameter sets are accepted if they result in a distance value less than or equal to $\delta^{R P E 1, *}$, the distance threshold. This allowed us to compute the relative probability, $p\left(H_{k}\right)$, for each hypothesis, as defined by the following equation

$$
p\left(H_{k} \mid \delta^{R P E 1, *}\right)=\frac{f\left(H_{k} \mid \delta^{R P E 1, *}\right)}{f\left(H_{1} \mid \delta^{R P E 1, *}\right)+f\left(H_{2} \mid \delta^{R P E 1, *}\right)^{\prime}}
$$

for $k \in\{1,2\}$. The results of the model selection analysis for RPE1 are shown in Figure $2 \mathrm{~d}$, where the relative probability of hypothesis 1 increases as $\delta^{R P E 1, *}$ tends to 0 , whilst the relative probability of hypothesis 2 decreases as a function of $\delta^{R P E 1, *}$. We hence concluded that the experimental data together with the mathematical models for HyplL-6 and IL-27 signaling provide greater support to hypothesis 1 (around $70 \%$ ) when compared to hypothesis 2 (around $30 \%)$. We note that as the distance threshold, $\delta^{R P E 1, *}$, is increased, both hypotheses become equally likely, as is to be expected. Given the results of the model selection, the Bayesian parameter inference for the mathematical models of HyplL-6 and IL-27 signaling was only carried out for hypothesis 1.

We used the ABC, sequential Monte Carlo (ABC-SMC), approach (96), to obtain posterior distributions for the parameters in Table 1, making use of the prior distributions in Table 2. All 
model parameters in Table 1 were estimated for the RPE1 data set. A subset of the parameters, which we would expect may vary with cell type, were then estimated for the Th-1 data set. In particular, the parameters not being estimated for Th-1 were sampled from the posterior distributions obtained via the ABC-SMC for RPE1, and those parameters estimated separately for Th-1 were: $q, d_{1}, d_{3}, \beta_{6}, \beta_{27},\left[R_{1}(0)\right],\left[R_{2}(0)\right],\left[S_{1}(0)\right]$ and $\left[S_{3}(0)\right]$.

To further validate the two mathematical models of cytokine signaling, we aimed to reproduce additional experimental results making use of the posterior parameter predictions from the RPE1 data ABC-SMC. Firstly, and in order to replicate the experimental dose response curve seen in Supp. Fig. 2a, we run both models using the $10^{4}$ accepted parameters sets from the ABC-SMC for 18 different values of cytokine concentration, within the range $\left[10^{-4}-10^{2}\right]$ log $\mathrm{nM}$. The results of this analysis are seen in Supp. Fig. 12b. We also modified the mathematical models to allow them to describe the IL-27Ra-GP130 chimera experiments (Fig. 3c). In particular, a new mathematical model for the chimera experiments was developed as follows: it consisted of the ODEs from the IL-27 model which are involved in the formation of the dimer, (Equations (23) - (26)) and the ODEs from the HyplL-6 model post-dimer formation (Equations (5) - (22)), in which $D_{6}$ was replaced by $D_{27}$. The ODE for the IL-27 induced dimer in the chimera model was as follows

$$
\begin{gathered}
\frac{d\left[D_{27}\right]}{d t}=r_{2,27}^{+}\left[C_{2}\right]\left[R_{1}\right]-r_{2,27}^{-}\left[D_{27}\right]-2 k_{1 a}^{+}\left[D_{27}\right]\left[S_{1}\right]+k_{1 a}^{-}\left(\left[S_{1} \cdot D_{27}\right]+\left[p S_{1} \cdot D_{27}\right]\right) \\
-2 k_{3 a}^{+}\left[D_{27}\right]\left[S_{3}\right]+k_{3 a}^{-}\left(\left[S_{3} \cdot D_{27}\right]+\left[p S_{3} \cdot D_{27}\right]\right)-\beta_{27}\left[D_{27}\right] .
\end{gathered}
$$

We simulated both the original mathematical model of IL-27 and the chimera model using the accepted parameter sets from the ABC-SMC. The results can be seen in Supp. Fig. 12a. Finally, we focussed on one of the mutant varieties of IL-27Ra, Y613F and sought to reproduce the results of Fig. 3b making use of the mathematical model of IL-27 signaling. Since the mutation decreases the affinity of STAT1 to IL-27R $\alpha$, we fixed the association and dissociation rates of STAT1 to the IL-27R chain, $k_{1 b}^{+}$and $k_{1 b}^{-}$, at values which resulted in a high $\mu \mathrm{M}$ affinity. The specific values chosen were $k_{1 b}^{+}=10^{-5} \mathrm{nM}^{-1} \mathrm{~s}^{-1}$ and $k_{1 b}^{-}=10^{1} \mathrm{~s}^{-1}$ which yields an affinity of $10^{2} \mu \mathrm{M}$. The rate $k_{1 b}^{-}$was chosen as approximately the median of the posterior distribution for this parameter from the ABC-SMC, and the rate $k_{1 b}^{+}$was then significantly decreased in order to increase the affinity value. We simulated the mathematical model of IL-27 signaling using the $10^{4}$ accepted parameter sets from the ABC-SMC, but where the rates $k_{1 b}^{+}$and $k_{1 b}^{-}$were fixed as described above. The pointwise medians and $95 \%$ credible intervals of these simulations are plotted in Supp. Fig. 12c, as well as the simulations for the WT, without altering any of the parameter values from the posterior distributions. Altering the binding affinity of STAT1 to IL-27Ra in this way in the mathematical model allows us to generate results which replicate reasonably well, the experimental observations for the Y613F mutant in Figure 3b.

\section{Live-cell dual-color single-molecule imaging studies:}

Single molecule imaging experiments were carried out by total internal reflection fluorescence (TIRF) microscopy with an inverted microscope (Olympus IX71) equipped with a triple-line total internal reflection (TIR) illumination condenser (Olympus) and a back-illuminated electron multiplied (EM) CCD camera (iXon DU897D, 512 x 512 pixel, Andor Technology) as recently described (38-40). A $150 \times$ magnification objective with a numerical aperture of 1.45 (UAPO 1503 /1.45 TIRFM, Olympus) was used for TIR illumination. All experiments were carried out at room temperature in medium without phenol red supplemented with an oxygen scavenger and a redox-active photoprotectant to minimize photobleaching (97). For Heterodimerization experiments of IL-27R $\alpha$ and GP130 cell surface labeling of RPE1 GP130 KO, co-transfected with $\mathrm{mXFPe}-\mathrm{IL}-27 \mathrm{R} \alpha$ and $\mathrm{mXFPm}-\mathrm{GP} 130$, was achieved by adding $\alpha \mathrm{GFP}-\mathrm{enNB} \mathrm{RHO}^{\mathrm{R} 1}$ and 
$\alpha$ GFP-miNB ${ }^{\text {DY647 }}$ to the medium at equal concentrations $(5 \mathrm{nM})$ and incubated for at least 5 min prior to stimulation with IL-27 $(20 \mathrm{nM})$ or HyplL-6 $(20 \mathrm{nM})$. For homodimerization experiments with mXFPm-GP130, $\alpha$ GFP-miNB ${ }^{\text {DY647 }}$ and $\alpha$ GFP-miNB ${ }^{\text {RHO11 }}$ (98) were used for cell surface receptor labelling as described above. The nanobodies were kept in the bulk solution during the whole experiment in order to ensure high equilibrium binding to mXFPGP130. For simultaneous dual color acquisition, $\alpha$ GFP-NB ${ }^{\text {RHO11 }}$ was excited by a $561 \mathrm{~nm}$ diode-pumped solid-state laser at $0.95 \mathrm{~mW}\left(\sim 32 \mathrm{~W} / \mathrm{cm}^{2}\right)$ and $\alpha \mathrm{GFP}-\mathrm{NB}^{\mathrm{DY} 647}$ by a $642 \mathrm{~nm}$ laser diode at $0.65 \mathrm{~mW}\left(\sim 22 \mathrm{~W} / \mathrm{cm}^{2}\right)$. Fluorescence was detected using a spectral image splitter (DualView, Optical Insight) with a 640 DCXR dichroic beam splitter (Chroma) in combination with the bandpass filter 585/40 (Semrock) for detection of RHO11 and 690/70 (Chroma) for detection of DY647 dividing each emission channel into 512x256 pixel. Image stacks of 150 frames were recorded at $32 \mathrm{~ms} /$ frame.

Single molecule localization and single molecule tracking were carried out using the multipletarget tracing (MTT) algorithm (99) as described previously (100). Step-length histograms were obtained from single molecule trajectories and fitted by two fraction mixture model of Brownian diffusion. Average diffusion constants were determined from the slope (2-10 steps) of the mean square displacement versus time lapse diagrams. Immobile molecules were identified by the density-based spatial clustering of applications with noise (DBSCAN) algorithm as described recently (101). For comparing diffusion properties and for co-tracking analysis, immobile particles were excluded from the data set. Prior to co-localization analysis, imaging channels were aligned with sub-pixel precision by using a spatial transformation. To this end, a transformation matrix was calculated based on a calibration measurement with multicolour fluorescent beads (TetraSpeck microspheres $0.1 \mathrm{~mm}$, Invitrogen) visible in both spectral channels (cp2tform of type 'affine', The MathWorks MATLAB 2009a).

Individual molecules detected in the both spectral channels were regarded as co-localized, if a particle was detected in both channels of a single frame within a distance threshold of $100 \mathrm{~nm}$ radius. For single molecule co-tracking analysis, the MTT algorithm was applied to this dataset of co-localized molecules to reconstruct co-locomotion trajectories (cotrajectories) from the identified population of co-localizations. For the co-tracking analysis, only trajectories with a minimum of 10 steps ( $\sim 320 \mathrm{~ms})$ were considered in order to robustly remove random receptor co-localizations (39). For heterodimerization experiments of $\mathrm{mXFPe-IL-27R \alpha}$

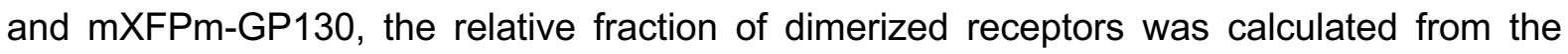
number of co-trajectories relative to the number of IL-27R $\alpha$ trajectories. GP130 was expressed in moderate excess ( 1.5-2 fold), so that maximal receptor assembly was not limited by abundance of the low-affinity subunit GP130.

For homodimerization experiments with GP130, the relative fraction of co-tracked molecules was determined with respect to the absolute number of trajectories and corrected for GP130 stochastically double-labelled with the same fluorophore species as follows:

$$
A B^{*}=\frac{A B}{2 \times\left[\left(\frac{A}{A+B}\right) \times\left(\frac{B}{A+B}\right)\right]} \text {, rel.co - locomotion }=\frac{2 \times A B^{*}}{(A+B)}
$$

where $A, B, A B$ and $A B^{*}$ are the numbers of trajectories observed for Rho11, DY647, cotrajectories and corrected co-trajectories, respectively.

The two-dimensional equilibrium dissociation constants $\left(K_{D}^{2 D}\right)$ were calculated according to the law of mass action for a monomer-dimer equilibrium:

Heterodimerization (IL-27R $\alpha+G P 130)$ : 


$$
K_{D}^{2 D}=\frac{([G P 130]-(\alpha \times[I L 27 R a])) \times([I L 27 R a]-(\alpha \times[I L 27 R a]))}{(\alpha \times[I L 27 R a])}
$$

or

$$
K_{D}^{2 D}=[G P 130] \times\left(\frac{1}{\alpha}-1\right)+[I L 27 R a] \times(\alpha-1)
$$

with: $\alpha=$ fraction of IL27 bound IL27Ra in complex with GP130

Homodimerization (GP130+GP130):

$$
\begin{aligned}
K_{D}^{2 D} & =\frac{[M]^{2}}{[D]}=\frac{\left([M]_{0}-2[D]\right)^{2}}{[D]} \\
K_{D}^{2 D} & =\frac{([G P 130]-2 \times(\alpha \times[G P 130]))^{2}}{2 \times(\alpha \times[G P 130])}
\end{aligned}
$$

with: $\alpha=$ fraction of GP130 homodimers relative to [GP130]/2

Where $[M]$ and $[D]$ are the concentrations of the monomer and the dimer, respectively, and $[M]_{0}$ is the total receptor concentration. 


\section{References:}

1. J. J. O'Shea, R. Plenge, JAK and STAT signaling molecules in immunoregulation and immune-mediated disease. Immunity 36, 542-550 (2012).

2. S. Pflanz et al., IL-27, a heterodimeric cytokine composed of EBI3 and p28 protein, induces proliferation of naive CD4+ T cells. Immunity 16, 779-790 (2002).

3. H. Yoshida, C. A. Hunter, The immunobiology of interleukin-27. Annu Rev Immunol 33, 417-443 (2015).

4. J. S. Stumhofer et al., Interleukin 27 negatively regulates the development of interleukin 17-producing $\mathrm{T}$ helper cells during chronic inflammation of the central nervous system. Nat Immunol 7, 937-945 (2006).

5. C. Diveu et al., IL-27 blocks RORc expression to inhibit lineage commitment of Th17 cells. J Immunol 182, 5748-5756 (2009).

6. D. C. Fitzgerald et al., Suppression of autoimmune inflammation of the central nervous system by interleukin 10 secreted by interleukin 27 -stimulated T cells. Nat Immunol $\mathbf{8}$, 1372-1379 (2007).

7. J. S. Stumhofer et al., Interleukins 27 and 6 induce STAT3-mediated T cell production of interleukin 10. Nat Immunol 8, 1363-1371 (2007).

8. C. Pot, L. Apetoh, A. Awasthi, V. K. Kuchroo, Induction of regulatory Tr1 cells and inhibition of T(H) 17 cells by IL-27. Semin Immunol 23, 438-445 (2011).

9. M. J. Boulanger, D. C. Chow, E. E. Brevnova, K. C. Garcia, Hexameric structure and assembly of the interleukin-6/IL-6 alpha-receptor/gp130 complex. Science 300, 21012104 (2003).

10. S. Rose-John, Interleukin-6 Family Cytokines. Cold Spring Harb Perspect Biol 10, (2018).

11. C. A. Hunter, S. A. Jones, IL-6 as a keystone cytokine in health and disease. Nature Immunology 16, 448-457 (2015).

12. T. Korn et al., IL-6 controls Th17 immunity in vivo by inhibiting the conversion of conventional T cells into Foxp3+ regulatory T cells. Proc Natl Acad Sci U S A 105, 18460-18465 (2008).

13. A. Kimura, T. Kishimoto, IL-6: regulator of Treg/Th17 balance. Eur J Immunol 40, 1830-1835 (2010).

14. G. W. Jones et al., Loss of CD4+ T cell IL-6R expression during inflammation underlines a role for IL- 6 trans signaling in the local maintenance of Th17 cells. $J$ Immunol 184, 2130-2139 (2010).

15. C. Rolvering et al., Crosstalk between different family members: IL27 recapitulates IFN gamma responses in HCC cells, but is inhibited by IL6-type cytokines. $\mathrm{Bba}-\mathrm{Mol}$ Cell Res 1864, 516-526 (2017).

16. A. P. Costa-Pereira et al., Mutational switch of an IL-6 response to an interferongamma-like response. P Natl Acad Sci USA 99, 8043-8047 (2002).

17. J. Schmitz, M. Weissenbach, S. Haan, P. C. Heinrich, F. Schaper, SOCS3 exerts its inhibitory function on interleukin-6 signal transduction through the SHP2 recruitment site of gp130. Journal of Biological Chemistry 275, 12848-12856 (2000).

18. H. Yasukawa et al., IL-6 induces an anti-inflammatory response in the absence of SOCS3 in macrophages. Nat Immunol 4, 551-556 (2003).

19. B. A. Croker et al., SOCS3 negatively regulates IL-6 signaling in vivo. Nat Immunol 4, 540-545 (2003).

20. C. Brender et al., Suppressor of cytokine signaling 3 regulates CD8 T-cell proliferation by inhibition of interleukins 6 and 27. Blood 110, 2528-2536 (2007). 
21. A. Camporeale, V. Poli, IL-6, IL-17 and STAT3: a holy trinity in auto-immunity? Front Biosci (Landmark Ed) 17, 2306-2326 (2012).

22. G. Regis, S. Pensa, D. Boselli, F. Novelli, V. Poli, Ups and downs: the STAT1:STAT3 seesaw of Interferon and gp130 receptor signalling. Semin Cell Dev Biol 19, 351-359 (2008).

23. S. Lucas, N. Ghilardi, J. Li, F. J. de Sauvage, IL-27 regulates IL-12 responsiveness of naive CD4(+) T cells through Stat1-dependent and -independent mechanisms. $P$ Natl Acad Sci USA 100, 15047-15052 (2003).

24. S. Kamiya et al., An indispensable role for STAT1 in IL-27-induced T-bet expression but not proliferation of naive CD4(+) T cells. Journal of Immunology 173, 3871-3877 (2004).

25. A. Takeda et al., Cutting edge: Role of IL-27/WSX-1 signaling for induction of T-Bet through activation of STAT1 during initial Th1 commitment. Journal of Immunology 170, 4886-4890 (2003).

26. C. Neufert et al., IL-27 controls the development of inducible regulatory T cells and Th17 cells via differential effects on STAT1. Eur J Immunol 37, 1809-1816 (2007).

27. T. Owaki et al., STAT3 is indispensable to IL-27-mediated cell proliferation but not to IL-27-induced Th1 differentiation and suppression of proinflammatory cytokine production. Journal of Immunology 180, 2903-2911 (2008).

28. K. Hirahara et al., Asymmetric Action of STAT Transcription Factors Drives Transcriptional Outputs and Cytokine Specificity. Immunity 42, 877-889 (2015).

29. S. Oniki et al., Interleukin-23 and interleukin-27 exert quite different antitumor and vaccine effects on poorly immunogenic melanoma. Cancer Res 66, 6395-6404 (2006).

30. M. Fischer et al., I. A bioactive designer cytokine for human hematopoietic progenitor cell expansion. Nat Biotechnol 15, 142-145 (1997).

31. H. H. Oberg, D. Wesch, S. Grussel, S. Rose-John, D. Kabelitz, Differential expression of CD126 and CD130 mediates different STAT-3 phosphorylation in CD4+CD25- and CD25high regulatory T cells. Int Immunol 18, 555-563 (2006).

32. P. O. Krutzik, M. R. Clutter, A. Trejo, G. P. Nolan, Fluorescent cell barcoding for multiplex flow cytometry. Curr Protoc Cytom Chapter 6, Unit 631 (2011).

33. U. A. Betz, W. Muller, Regulated expression of gp130 and IL-6 receptor alpha chain in T cell maturation and activation. Int Immunol 10, 1175-1184 (1998).

34. J. Martinez-Fabregas et al., Kinetics of cytokine receptor trafficking determine signaling and functional selectivity. Elife 8, (2019).

35. C. Gorby et al., Engineered IL-10 variants elicit potent immunomodulatory effects at low ligand doses. Sci Signal 13, (2020).

36. V. Ruprecht, Weghuber, J., Wieser, S., Schütz, G. J, in Advances in Planar Lipid Bilayers and Liposomes. (2010), vol. 12,, pp. 21-40.

37. I. Moraga et al., Instructive roles for cytokine-receptor binding parameters in determining signaling and functional potency. Science Signaling 8, (2015).

38. S. Wilmes et al., Receptor dimerization dynamics as a regulatory valve for plasticity of type I interferon signaling. J Cell Biol 209, 579-593 (2015).

39. S. Wilmes et al., Mechanism of homodimeric cytokine receptor activation and dysregulation by oncogenic mutations. Science 367, 643-652 (2020).

40. I. Moraga et al., Tuning Cytokine Receptor Signaling by Re-orienting Dimer Geometry with Surrogate Ligands. Cell 160, 1196-1208 (2015).

41. S. Pflanz et al., WSX-1 and glycoprotein 130 constitute a signal-transducing receptor for IL-27. J Immunol 172, 2225-2231 (2004). 
42. M. Wiederkehr-Adam et al., Characterization of phosphopeptide motifs specific for the Src homology 2 domains of signal transducer and activator of transcription 1 (STAT1) and STAT3. J Biol Chem 278, 16117-16128 (2003).

43. A. Pradhan, Q. T. Lambert, L. N. Griner, G. W. Reuther, Activation of JAK2-V617F by components of heterodimeric cytokine receptors. J Biol Chem 285, 16651-16663 (2010).

44. H. Kim, T. S. Hawley, R. G. Hawley, H. Baumann, Protein tyrosine phosphatase 2 (SHP-2) moderates signaling by gp130 but is not required for the induction of acutephase plasma protein genes in hepatic cells. Mol Cell Biol 18, 1525-1533 (1998).

45. D. W. Huang, B. T. Sherman, R. A. Lempicki, Systematic and integrative analysis of large gene lists using DAVID bioinformatics resources. Nat Protoc 4, 44-57 (2009).

46. J. Bancerek et al., CDK8 kinase phosphorylates transcription factor STAT1 to selectively regulate the interferon response. Immunity 38, 250-262 (2013).

47. S. Rutz et al., Deubiquitinase DUBA is a post-translational brake on interleukin-17 production in T cells. Nature 518, 417-421 (2015).

48. K. L. O'Hagan, S. D. Miller, H. Phee, Pak2 is essential for the function of Foxp3+regulatory T cells through maintaining a suppressive Treg phenotype. Sci RepUk 7, (2017).

49. D. Z. Ye, J. Field, PAK signaling in cancer. Cell Logist 2, 105-116 (2012).

50. Y. Liao, J. Wang, E. J. Jaehnig, Z. Shi, B. Zhang, WebGestalt 2019: gene set analysis toolkit with revamped UIs and APIs. Nucleic Acids Res 47, W199-W205 (2019).

51. J. Satoh, H. Tabunoki, A Comprehensive Profile of ChIP-Seq-Based STAT1 Target Genes Suggests the Complexity of STAT1-Mediated Gene Regulatory Mechanisms. Gene Regul Syst Bio 7, 41-56 (2013).

52. I. Rusinova et al., Interferome v2.0: an updated database of annotated interferonregulated genes. Nucleic Acids Res 41, D1040-1046 (2013).

53. H. N. Suh et al., Role of interleukin-6 in the control of DNA synthesis of hepatocytes: involvement of PKC, p44/42 MAPKs, and PPARdelta. Cell Physiol Biochem 22, 673684 (2008).

54. A. V. Villarino et al., IL-27 limits IL-2 production during Th1 differentiation. $J$ Immunol 176, 237-247 (2006).

55. K. Hirahara et al., Interleukin-27 Priming of T Cells Controls IL-17 Production In trans via Induction of the Ligand PD-L1. Immunity 36, 1017-1030 (2012).

56. X. Hu et al., Sensitization of IFN-gamma Jak-STAT signaling during macrophage activation. Nat Immunol 3, 859-866 (2002).

57. V. Francois-Newton, M. Livingstone, B. Payelle-Brogard, G. Uze, S. Pellegrini, USP18 establishes the transcriptional and anti-proliferative interferon alpha/beta differential. Biochem J 446, 509-516 (2012).

58. K. Zenke, M. Muroi, K. I. Tanamoto, IRF1 supports DNA binding of STAT1 by promoting its phosphorylation. Immunol Cell Biol 96, 1095-1103 (2018).

59. K. Karwacz et al., Critical role of IRF1 and BATF in forming chromatin landscape during type 1 regulatory cell differentiation. Nat Immunol 18, 412-421 (2017).

60. A. Yoshimura, Y. Wakabayashi, T. Mori, Cellular and molecular basis for the regulation of inflammation by TGF-beta. J Biochem 147, 781-792 (2010).

61. A. Awasthi et al., A dominant function for interleukin 27 in generating interleukin 10producing anti-inflammatory T cells. Nat Immunol 8, 1380-1389 (2007).

62. J. B. Brown et al., P-selectin glycoprotein ligand-1 is needed for sequential recruitment of T-helper 1 (Th1) and local generation of Th17 T cells in dextran sodium sulfate (DSS) colitis. Inflamm Bowel Dis 18, 323-332 (2012). 
63. M. Matsumoto et al., CD43 collaborates with P-selectin glycoprotein ligand-1 to mediate E-selectin-dependent $\mathrm{T}$ cell migration into inflamed skin. $J$ Immunol 178, 2499-2506 (2007).

64. D. N. Slenter et al., WikiPathways: a multifaceted pathway database bridging metabolomics to other omics research. Nucleic Acids Res 46, D661-D667 (2018).

65. A. Petretto et al., Proteomic analysis uncovers common effects of IFN-gamma and IL27 on the HLA class I antigen presentation machinery in human cancer cells. Oncotarget 7, 72518-72536 (2016).

66. L. H. Wong, I. Hatzinisiriou, R. J. Devenish, S. J. Ralph, IFN-gamma priming upregulates IFN-stimulated gene factor 3 (ISGF3) components, augmenting responsiveness of IFN-resistant melanoma cells to type I IFNs. J Immunol 160, 54755484 (1998).

67. M. Tokuyama et al., ERVmap analysis reveals genome-wide transcription of human endogenous retroviruses. Proc Natl Acad Sci U S A 115, 12565-12572 (2018).

68. C. Garbers et al., Plasticity and cross-talk of interleukin 6-type cytokines. Cytokine Growth Factor Rev 23, 85-97 (2012).

69. S. Kang, M. Narazaki, H. Metwally, T. Kishimoto, Historical overview of the interleukin-6 family cytokine. J Exp Med 217, (2020).

70. R. Umeshita-Suyama et al., Characterization of IL-4 and IL-13 signals dependent on the human IL-13 receptor alpha chain 1: redundancy of requirement of tyrosine residue for STAT3 activation. Int Immunol 12, 1499-1509 (2000).

71. O. W. Nadeau et al., The proximal tyrosines of the cytoplasmic domain of the beta chain of the type I interferon receptor are essential for signal transducer and activator of transcription (Stat) 2 activation. Evidence that two Stat 2 sites are required to reach a threshold of interferon alpha-induced Stat 2 tyrosine phosphorylation that allows normal formation of interferon-stimulated gene factor 3. J Biol Chem 274, 4045-4052 (1999).

72. M. N. Sharif et al., IFN-alpha priming results in a gain of proinflammatory function by IL-10: implications for systemic lupus erythematosus pathogenesis. $J$ Immunol 172, 6476-6481 (2004).

73. D. Richter et al., Ligand-induced type II interleukin-4 receptor dimers are sustained by rapid re-association within plasma membrane microcompartments. Nat Commun 8, 15976 (2017).

74. J. P. Twohig et al., Activation of naive CD4(+) T cells re-tunes STAT1 signaling to deliver unique cytokine responses in memory CD4(+) T cells. Nat Immunol 20, 458470 (2019).

75. P. C. Heinrich et al., Principles of interleukin (IL)-6-type cytokine signalling and its regulation. Biochem J 374, 1-20 (2003).

76. D. Levin, D. Harari, G. Schreiber, Stochastic receptor expression determines cell fate upon interferon treatment. Mol Cell Biol 31, 3252-3266 (2011).

77. I. Moraga, D. Harari, G. Schreiber, G. Uze, S. Pellegrini, Receptor density is key to the alpha2/beta interferon differential activities. Mol Cell Biol 29, 4778-4787 (2009).

78. C. C. M. Ho et al., Decoupling the Functional Pleiotropy of Stem Cell Factor by Tuning c-Kit Signaling. Cell 168, 1041-1052 e1018 (2017).

79. P. Charlot-Rabiega, E. Bardel, C. Dietrich, R. Kastelein, O. Devergne, Signaling events involved in interleukin 27 (IL-27)-induced proliferation of human naive CD4+ T cells and B cells. J Biol Chem 286, 27350-27362 (2011).

80. J. Diegelmann, T. Olszak, B. Goke, R. S. Blumberg, S. Brand, A Novel Role for Interleukin-27 (IL-27) as Mediator of Intestinal Epithelial Barrier Protection Mediated via Differential Signal Transducer and Activator of Transcription (STAT) Protein 
Signaling and Induction of Antibacterial and Anti-inflammatory Proteins. Journal of Biological Chemistry 287, 286-298 (2012).

81. H. Bender et al., Interleukin-27 displays interferon-gamma-like functions in human hepatoma cells and hepatocytes. Hepatology 50, 585-591 (2009).

82. T. Imamichi, J. Yang, W. Huang da, B. Sherman, R. A. Lempicki, Interleukin-27 induces interferon-inducible genes: analysis of gene expression profiles using Affymetrix microarray and DAVID. Methods Mol Biol 820, 25-53 (2012).

83. J. M. Fakruddin et al., Noninfectious papilloma virus-like particles inhibit HIV-1 replication: implications for immune control of HIV-1 infection by IL-27. Blood 109, 1841-1849 (2007).

84. A. C. Frank et al., Interleukin-27, an anti-HIV-1 cytokine, inhibits replication of hepatitis C virus. J Interferon Cytokine Res 30, 427-431 (2010).

85. S. L. LaPorte et al., Molecular and structural basis of cytokine receptor pleiotropy in the interleukin-4/13 system. Cell 132, 259-272 (2008).

86. J. B. Spangler, I. Moraga, K. M. Jude, C. S. Savvides, K. C. Garcia, A strategy for the selection of monovalent antibodies that span protein dimer interfaces. J Biol Chem 294, 13876-13886 (2019).

87. A. Kirchhofer et al., Modulation of protein properties in living cells using nanobodies. Nat Struct Mol Biol 17, 133-138 (2010).

88. M. C. Hochberg, Updating the American College of Rheumatology revised criteria for the classification of systemic lupus erythematosus. Arthritis Rheum 40, 1725 (1997).

89. J. Cox, M. Mann, MaxQuant enables high peptide identification rates, individualized p.p.b.-range mass accuracies and proteome-wide protein quantification. Nat Biotechnol 26, 1367-1372 (2008).

90. J. Cox et al., Andromeda: a peptide search engine integrated into the MaxQuant environment. J Proteome Res 10, 1794-1805 (2011).

91. P. O. Krutzik, G. P. Nolan, Fluorescent cell barcoding in flow cytometry allows highthroughput drug screening and signaling profiling. Nat Methods 3, 361-368 (2006).

92. W. Huang da, B. T. Sherman, R. A. Lempicki, Bioinformatics enrichment tools: paths toward the comprehensive functional analysis of large gene lists. Nucleic Acids Res 37, 1-13 (2009).

93. W. Huang da, B. T. Sherman, R. A. Lempicki, Systematic and integrative analysis of large gene lists using DAVID bioinformatics resources. Nat Protoc 4, 44-57 (2009).

94. N. Kozer et al., Exploring higher-order EGFR oligomerisation and phosphorylation--a combined experimental and theoretical approach. Mol Biosyst 9, 1849-1863 (2013).

95. D. N. Itzhak, S. Tyanova, J. Cox, G. H. Borner, Global, quantitative and dynamic mapping of protein subcellular localization. Elife 5, (2016).

96. T. Toni, D. Welch, N. Strelkowa, A. Ipsen, M. P. Stumpf, Approximate Bayesian computation scheme for parameter inference and model selection in dynamical systems. J R Soc Interface 6, 187-202 (2009).

97. J. Vogelsang et al., A reducing and oxidizing system minimizes photobleaching and blinking of fluorescent dyes. Angew Chem Int Ed Engl 47, 5465-5469 (2008).

98. A. Kirchhofer et al., Modulation of protein properties in living cells using nanobodies. Nat Struct Mol Biol 17, 133-U162 (2010).

99. A. Serge, N. Bertaux, H. Rigneault, D. Marguet, Dynamic multiple-target tracing to probe spatiotemporal cartography of cell membranes. Nat Methods 5, 687-694 (2008).

100. C. You et al., Receptor dimer stabilization by hierarchical plasma membrane microcompartments regulates cytokine signaling. Sci Adv 2, e1600452 (2016).

101. F. Roder, A. Lubk, D. Wolf, T. Niermann, Noise estimation for off-axis electron holography. Ultramicroscopy 144, 32-42 (2014). 
bioRxiv preprint doi: https://doi.org/10.1101/2021.01.08.425379; this version posted January 9, 2021. The copyright holder for this preprint (which was not certified by peer review) is the author/funder, who has granted bioRxiv a license to display the preprint in perpetuity. It is made available under aCC-BY 4.0 International license. 


\section{FIGURE LEGENDS:}

\section{Figure 1 Cytokine receptor activation by IL-27 and (Hyp)IL-6:}

a) Cartoon model of stepwise assembly of the IL-27 and HyplL-6-induced receptor complex and subsequent activation of STAT1 and STAT3.

b) Dose-dependent phosphorylation of STAT1 and STAT3 as a response to IL-27 and HyplL-6 stimulation in TH-1 cells, normalized to maximal IL-27 stimulation. Data was obtained from three biological replicates with each two technical replicates, showing mean \pm std dev.

c) Phosphorylation kinetics of STAT1 and STAT3 followed after stimulation with saturating concentrations of IL-27 (2nM) and HyplL-6 (20nM) or unstimulated TH-1 cells, normalized to maximal IL-27 stimulation. Data was obtained from five biological replicates with each two technical replicates, showing mean \pm std dev.

d) Top: Phosphorylation kinetics of STAT1 and STAT3 followed after stimulation with HyplL-6 (20nM) or left unstimulated, comparing wt RPE1 and RPE1 GP130KO reconstituted with high levels of $\mathrm{mXFPm}-\mathrm{GP} 130$ (=10x [GP130]). Data was normalized to maximal stimulation levels of wt RPE1. Left: cell surface GP130 levels comparing RPE1 GP130KO, wt RPE1 and RPE1 GP130KO stably expressing mXFPm-GP130 measured by flow cytometry. Data was obtained from one biological replicate with each two technical replicates, showing mean \pm std dev. Bottom right: cell surface levels of GP130 measured by flow cytometry for indicated cell lines.

e) Cartoon model of cell surface labeling of mXFP-tagged receptors by dye-conjugated anti-GFP nanobodies (NB) and identification of receptor dimers by single molecule dual-colour co-localization.

f) Raw data of dual-colour single-molecule TIRF imaging of mXFPe-IL-27Ra ${ }^{\mathrm{NB}-\mathrm{RHO} 11}$ and GP130 ${ }^{\text {NB-DY649 }}$ after stimulation with IL-27. Particles from the insets (IL-27Ra: red \& GP130: blue) were followed by single molecule tracking (150 frames $\sim 4.8 \mathrm{~s}$ ) and trajectories $>10$ steps $(320 \mathrm{~ms})$ are displayed. Receptor heterodimerization was detected by co-localization/co-tracking analysis.

g) Relative number of co-trajectories observed for heterodimerization of IL-27Ra and GP130 as well as homodimerization of GP130 for unstimulated cells or after indicated cytokine stimulation. Each data point represents the analysis from one cell with a minimum of 23 cells measured for each condition. ${ }^{*} P<0.05$, ${ }^{* *} P \leq 0.01$, ${ }^{* * *} P \leq 0.001$; n.s., not significant.

h) Stoichiometry of the IL-27-induced receptor complex revealed by bleaching analysis. Left: Intensity traces of mXFPe-IL27Ra ${ }^{\mathrm{NB}-\mathrm{RHO} 11}$ and GP130 ${ }^{\mathrm{NB}-\mathrm{DY} 649}$ were followed until fluorophore bleaching. Middle: Merged imaging raw data for selected timepoints. Right: overlay of the trajectories for IL-27Ra (red) and GP130 (blue).

\section{Figure 2: Mathematical modelling results in RPE1 and Th-1 cells.}

a) Simplified cartoon model of IL-27/HyplL-6 signal propagation layers and coverage of the mathematical modelling approach.

b) Model selection results showing the relative probabilities of each hypothesis, for different values of the distance threshold, $\delta^{*}$, in RPE1 cells.

c) Pointwise median and $95 \%$ credible intervals of the predictions from the mathematical model, calibrated with the experimental data, using the posterior distributions for the parameters from the ABC-SMC. 
d) Kernel density estimates of the posterior distributions for the parameters $p \in$ $\left\{r_{1, j}^{+}, r_{1, j}^{-}, r_{2, j}^{+}, r_{2, j}^{-}, k_{i a}^{+}, k_{i a}^{-}, k_{i b}^{+}, k_{i b}^{-}, q, d_{i}, \beta_{j},\left[R_{1}(0)\right],\left[R_{2}(0)\right],\left[S_{1}(0)\right],\left[S_{3}(0)\right]\right\} \quad$ in $\quad$ the mathematical models where $j \in\{6,27\}$ and $i \in\{1,3\}$.

\section{Figure 3: IL-27Ra cytoplasmic domain is required for sustained pSTAT1 kinetics.}

a) Representation of the cytoplasmic domain of IL-27Ra with its highlighted tyrosine residues $Y 543$ and $Y 613$.

b) STAT1 and STAT3 phosphorylation kinetics of RPE1 clones stably expressing wt and mutant IL-27R $\alpha$ after stimulation with IL-27 (10 nM, top panels) or after stimulation with HypIL-6 (20 nM, bottom panels), normalized to maximal levels of wt IL-27Ra stimulated with IL-27 (top) or HyplL-6 (bottom). Data was obtained from three experiments with each two technical replicates, showing mean \pm std dev. Bottom right: cell surface levels variants measured by flow cytometry for indicated IL-27Ra cell lines.

c) Cytoplasmic domain of IL-27Ra is required for sustained pSTAT1 activation. Left: Cartoon representation of receptor complexes. Right: STAT1 and STAT3 phosphorylation kinetics of RPE1 clones stably expressing wt IL-27Ra and IL-27RaGP130 chimera after stimulation with IL-27 (10 nM, top panels) or after stimulation with HyplL-6 (20 nM, bottom panels). Data was normalized to maximal levels for each cytokine and cell line. Data was obtained from two experiments with each 2 technical replicates, showing mean \pm std dev.

d) Phosphatases do not account for differential pSTAT1/3 activity induced by IL-27 and HyplL-6. Left: Schematic representation of workflow using JAK inhibitor Tofacitinib. Right: MFI ratio of Tofacitinib-treated and non-treated RPE1 mXFPe-IL-27Ra cells for pSTAT1 and pSTAT3 after stimulation with IL-27 (10nM) and HyplL-6 (20nM). Data was obtained from two experiments with each two technical replicates, showing mean \pm std dev.

Figure 4: Unique and overlapping effects of IL-27 and HyplL-6 on the phosphoproteome of Th-1 cells.

a) Volcano plot of the phospho-sites regulated ( $p$ value $\leq 0.05$, fold change $\geq+1.5$ or $\leq-$ $1.5)$ by IL-27 (left) and HyplL-6 (right). Data was obtained from three biological replicates.

b) Heatmap representation (examples) of shared and differentially up- (left) and downregulated (right) phospho-sites after IL-27 and HyplL-6 stimulation. Data represents the mean $\left(\log _{2}\right)$ fold change of three biological replicates.

c) Tyrosine and Serine phosphorylation of selected STAT proteins after stimulation with IL-27 (red) and HyplL-6 (blue). ${ }^{*} \mathrm{P}<0.05,{ }^{* *} \mathrm{P} \leq 0.01$, ${ }^{* * *} \mathrm{P} \leq 0.001$; n.s., not significant.

d) pS727-STAT1 and pS727-STAT3 phosphorylation kinetics in Th-1 cells after stimulation with IL-27 or HyplL-6, normalized to maximal IL-27 stimulation. Data was obtained from three biological replicates with each two technical replicates, showing mean \pm std dev.

e) GO analysis "biological processes" of the phospho-sites regulated by IL-27 (red) and HyplL-6 (blue) represented as bubble-plots.

f) Phosphorylation of target proteins associated with STAT3/CDK transcription initiation complex after stimulation with IL-27 (blue) and HyplL-6 (red) and schematic representation of transcription regulation of RNA polymerase II with identified phospho-sites (red flags). 
Figure 5: Kinetic decoupling of gene induction programs depends on sustained STAT1 activation by IL-27.

a) Principal component analysis for genes found to be significantly upregulated (left) or downregulated (right) for at least one of the tested conditions (time \& cytokine). Data was obtained from three biological replicates.

b) Kinetics of gene induction shared between IL-27 and HyplL-6 (relative to IL-27) for upregulated genes (red) or downregulated genes (green).

c) Kinetics of gene numbers induced after IL-27 and HyplL-6 stimulation for upregulated genes (left) and downregulated genes (right).

d) GSEA reactome analysis of selected pathways with significantly altered gene induction in response to IL-27 or HyplL-6 stimulation. Data represents the mean $\left(\log _{2}\right)$ fold change of three biological replicates.

e) Cluster analysis comparing the gene induction kinetics after IL-27 or HyplL-6 stimulation. Gene induction heatmaps for example genes as well as induction kinetics (mean) are shown for highlighted gene clusters. Data represents the mean $\left(\log _{2}\right)$ fold change of three biological replicates.

\section{Figure 6: IL-27-induced upregulation of IRF1 amplifies induction of STAT1-dependent genes}

a) Kinetics of IRF1 protein expression as a response to continuous IL-27 and HypIL-6 stimulation in Th-1 cells. Data was obtained from three biological replicates with each two technical replicates, showing mean \pm std dev. Dotted line indicates baseline level.

b) Kinetics of IRF1 protein expression and siRNA-mediated IRF1 knockdown in RPE1 IL$27 R \alpha$ cells stimulated with IL-27 $(2 \mathrm{nM})$. Data was obtained from one representative experiment with each two technical replicates, normalized to maximal IRF1 induction (6h), showing mean \pm std dev.

c) Kinetics of STAT1 (left) and STAT3 (right) phosphorylation after siRNA-mediated IRF1 knockdown in RPE1 IL-27Ra cells stimulated with IL-27 (2nM). Data was obtained from one representative experiment with each two technical replicates, showing mean \pm std dev.

d) Kinetics of gene induction (STAT1, GBP5, OAS1, SOCS3) followed by RT qPCR in RPE1 IL-27R $\alpha$ cells stimulated with IL-27 (2nM) with and without siRNA-mediated knockdown of IRF1. Data was obtained from three experiments with each two technical replicates, showing mean \pm SEM.

Figure 7: IL-27-induced STAT1 response drives global proteomic changes in Th-1 cells.

a) Workflow for quantitative SILAC proteomic analysis of Th-1 cells continuously stimulated $(24 \mathrm{~h})$ with IL-27 (10nM), HyplL-6 (20nM) or left untreated.

b) Global proteomic changes in Th-1 cells induced by IL-27 (left) or HyplL-6 (right) represented as volcano plots. Proteins significantly up- or downregulated are highlighted in red ( $p$ value $\leq 0.05$, fold change $\geq+1.5$ or $\leq-1.5$ ). Significantly altered ISG-encoded proteins by IL-27 are highlighted in yellow. Data was obtained from three biological replicates.

c) Venn diagrams comparing unique upregulated (left) and downregulated (right) proteins by IL-27 (blue) and HyplL-6 (red) as well as shared altered proteins. ISG-encoded proteins are highlighted in yellow. 
d) Heatmaps of the top 30 up- and downregulated proteins by IL-27 compared to HyplL6. Data representation of the mean $\left(\log _{2}\right)$ fold change of three biological replicates.

e) Heatmap representation and enrichment plot of proteins identified by GSEA reactome pathway enrichment analysis "Cytokine signaling and immune system" induced by IL27 . Data representation of the mean $\left(\log _{2}\right)$ fold change of three biological replicates.

f) Correlation of IL-27 and HypIL-6-induced RNA-seq transcript levels ( $\geq+2$ or $\leq-2 \mathrm{fc}$ ) with quantitative proteomic data $\left(\geq+1.5\right.$ or $\leq-1.5 \mathrm{fc}$ ). Data representation of the mean $\left(\log _{2}\right)$ fold change of three biological replicates.

\section{Figure 8: Receptor and STAT concentrations determine the nature of the cytokine response.}

a) Copy numbers of indicated proteins determined for different T-cell subsets using massspectrometry based proteomics (ImmPRes - http://immpres.co.uk).

b) Model predictions for varying levels of STAT1 and STAT3 (left panel) or IL-27Ra and GP130 (right panel) for phosphorylation kinetics of STAT1 and STAT3.

c) Gene expression profiles determined by RNAseq analysis comparing indicated genes of a cohort of SLE risk patients with a cohort of healthy controls. Data obtained from: Proc Natl Acad Sci U S A 115, 12565-12572 . *P <0.05, **P $\leq 0.01,{ }^{* * *} \mathrm{P} \leq 0.001$; n.s., not significant.

d) Dose-dependent phosphorylation of STAT1 and STAT3 as a response to IL-27 and HyplL-6 stimulation in naive and IFNa2-primed (2nM, 24h) Th-1 cells, normalized to maximal IL-27 stimulation (ctrl). Data was obtained from four biological replicates with each two technical replicates, showing mean \pm std dev.

e) Phosphorylation of STAT1 (left) and STAT3 (right) as a response to IL-27 (2nM, 15min) and HyplL-6 (10nM, 15min) stimulation in healthy control (ctrl) and SLE patient CD4+ T-cells. Data was obtained from five healthy control donors (5) and six SLE patients. ${ }^{*} \mathrm{P}<0.05,{ }^{* *} \mathrm{P} \leq 0.01,{ }^{* *} \mathrm{P} \leq 0.001$; n.s., not significant.

f) Tofacitinib titration to inhibit STAT1 and STAT3 phosphorylation by HyplL-6 (10nM, 15min) in Th-1 cells (left) and RPE1 cells stably expressing wt IL-27Ra (right).

\section{Supp. Figure 1:}

a) Comparison of dose-dependent phosphorylation (STAT1/3) of purchased IL-27 and mIL-27sc in activated CD4+ cells, normalized to maximal MFI levels. Data was obtained from one (purchased) or two (mIL-27sc) biological replicates with each two technical replicates, showing mean \pm std dev.

b) Schematic workflow of T-cell isolation, TH1 differentiation, fluorescence barcoding and gating strategy for high throughput flow cytometry.

c) Phosphorylation kinetics of STAT1 and STAT3 followed after stimulation with IL-27 (10nM) and HyplL-6 (20nM) or unstimulated TH1 cells. Data (from Fig. 1c) was normalized to maximal MFI levels for each cytokine. Data was obtained from five biological replicates with each two technical replicates, showing mean \pm std dev.

d) Phosphorylation kinetics of activated PBMCs (CD4+, CD8+) of STAT1 and STAT3 followed after stimulation with IL-27 (2nM) and HyplL-6 (20nM) or unstimulated cells. Data was normalized to maximal IL-27 stimulation. Data was obtained from two biological replicates with each two technical replicates, showing mean \pm std dev.

e) Dose-response experiments in wt RPE1 cells for pSTAT1 (left) and pSTAT3 (right), stimulated with IL-27 or HypIL-6, normalized to maximal HyplL-6 stimulation. Data was 
obtained from one representative experiment with each two technical replicates, showing mean \pm std dev.

\section{Supp. Figure 2:}

a) Dose-response experiments for pSTAT1 and pSTAT3 comparing RPE1 GP130 KO cells (left), wt RPE1 (middle) and RPE1 mXFPe-IL27Ra (right) after stimulation with IL-27 or HyplL-6, normalized to maximal HyplL-6 stimulation. Data was obtained from one representative experiment with each two technical replicates, showing mean \pm std dev.

b) Ligand-induced receptor dimerization: Top panel: Dual-colour co-tracking of IL-27Ra and GP130 in the absence (top) and presence (bottom) of IL-27 (20nM). Trajectories (150 frames, $\sim 4.8 \mathrm{~s}$ ) of individual mXFPe-IL27Ra ${ }^{\mathrm{NB}-\mathrm{RHO} 11}$ (red) and GP130 ${ }^{\mathrm{NB}-\mathrm{DY} 649}$ (blue) and co-trajectories (magenta) are shown for a representative cell. Bottom panel: Dual-colour co-tracking of GP130 in the absence (top) and presence (bottom) of HyplL-6 (20nM). Trajectories (150 frames, $\sim 4.8 \mathrm{~s}$ ) of individual mXFPe-IL27Ra ${ }^{\mathrm{NB}-\mathrm{RHO} 11}$ (red) and GP130 ${ }^{\mathrm{NB}-\mathrm{DY} 649}$ (blue) and co-trajectories (magenta) are shown for a representative cell.

c) Top: Cartoon model of cell surface labeling of mXFP-tagged GP130 by dye-conjugated anti-GFP nanobodies (NB) and formation of single-colour homodimers (left) or dualcolour homodimers (right). Below: Examples for intensity traces of single-colour dualstep bleaching (left) or dual-colour single-step bleaching (right). Insets show raw data for selected timepoints and corresponding trajectories.

d) Top: comparison of diffusion coefficients (D) for mXFPe-IL-27Ra ${ }^{\mathrm{NB}-\mathrm{RHO} 11}$ (red) and mXFPmGP130 13 -DY649 (blue) in presence and absence of IL-27 stimulation (20nM), as well as co-trajectories after IL-27 stimulation (magenta). Bottom: comparison of diffusion coefficients for mXFPm-GP130 ${ }^{\mathrm{NB}-\mathrm{RHO} 11}$ (red) in presence and absence of HyplL-6 stimulation (20nM), as well as co-trajectories after HyplL-6 stimulation (magenta). Each data point represents the analysis from one cell with a minimum of 23 cells measured for each condition. ${ }^{*} P<0.05$, ${ }^{* *} P \leq 0.01$, ${ }^{* * *} P \leq 0.001$; n.s., not significant.

\section{Supp. Figure 3:}

a) Reactions involving ligand binding and dimerization in the HyplL-6 model.

b) Reactions involving ligand binding and dimerization in the IL-27 model.

c) Reactions involving the STAT molecules $\left(S_{j}\right.$ for $j \in\{1,3\}$ ) in the HyplL-6 model.

d) Reactions involving the STAT molecules $\left(S_{j}\right.$ for $\left.j \in\{1,3\}\right)$ in the IL-27 model.

e) Reactions involving receptor internalisation/degradation in the HyplL-6 model. Here $H 1=\beta_{6}$ and $H 2=\gamma_{6}([p S 1]+[p S 1])$.

f) Reactions involving receptor internalisation/degradation in the IL-27 model. Here $H 1=$ $\beta_{27}$ and $H 2=\gamma_{27}([p S 1]+[p S 1])$.

g) Dephosphorylation of $\left(S_{j}\right.$ for $\left.j \in\{1,3\}\right)$ in the cytoplasm. This reaction occurs in both models.

h) Key for the molecules in the reactions.

\section{Supp. Figure 4:}

a) STAT1 (left) and STAT3 (right) phosphorylation kinetics of RPE1 clones stably expressing wt IL-27R $\alpha$ after stimulation with IL-27 or after stimulation with HypIL-6 
normalized to maximal IL-27 stimulation. Data was obtained from three experiments with each two technical replicates, showing mean \pm std dev.

b) Dose-response experiments for pSTAT1 (left) and pSTAT3 (right) in RPE1 cells stably expressing wt IL-27R $\alpha$ or tyrosine-mutants after stimulation with IL-27, normalized to maximal stimulation of wt IL-27Ra. Data was obtained from one representative experiment with each two technical replicates, showing mean \pm std dev.

\section{Supp. Figure 5:}

a) Dose-response experiments for pSTAT1 (left) and pSTAT3 (right) in RPE1 cells stably expressing wt IL-27Ra or IL-27Ra-GP130 chimera after stimulation with IL-27. Data normalized to maximal stimulation of wt IL-27Ra. Data was obtained from one representative experiment with each two technical replicates, showing mean \pm std dev.

b) STAT1 (left) and STAT3 (right) phosphorylation kinetics in RPE1 IL-27Ra cells stimulated with IL-27 or HyplL-6 with and without JAK inhibition by Tofacitinib. Data was normalized to maximal IL-27 stimulation. Data was obtained from two experiments with each two technical replicates, showing mean \pm std dev.

c) STAT1 (left) and STAT3 (right) phosphorylation kinetics in Th-1 cells stimulated with IL-27 or HypIL-6 with and without JAK inhibition by Tofacitinib. Data was normalized to to maximal IL-27 stimulation. Data was obtained from two biological replicates with each two technical replicates, showing mean \pm std dev.

d) MFI ratio of Tofacitinib-treated and non-treated Th-1 cells for pSTAT1 (left) and pSTAT3 (right) after stimulation with IL-27 (10nM) and HyplL-6 (20nM). Data was obtained from two biological replicates with each two technical replicates, showing mean \pm std dev.

\section{Supp. Figure 6:}

a) STAT1 (left) and STAT3 (right) phosphorylation kinetics in RPE1 IL-27Ra cells stimulated with IL-27 or HyplL-6 with and without pretreatment with cycloheximide $(\mathrm{CHX})$. Data was normalized to to maximal IL-27 stimulation. Data was obtained from two experiments with each two technical replicates, showing mean \pm std dev.

b) STAT1 (left) and STAT3 (right) phosphorylation kinetics in TH1 cells stimulated with IL-27 or HyplL-6 with and without pretreatment with cycloheximide (CHX). Data was normalized to to maximal IL-27 stimulation. Data was obtained from two biological replicates with each two technical replicates, showing mean \pm std dev.

\section{Supp. Figure 7:}

a) Workflow for quantitative SILAC phospho-proteomic analysis of $\mathrm{TH}-1$ cells stimulated (15min) with IL-27 (10 nM), HyplL-6 (20 nM) or left untreated.

b) Schematic representation of the main GO terms regulated by IL27 as inferred from our p-proteomics studies. Red represents downregulated p-sites and blue represents upregulated p-sites upon IL27 stimulation of human primary Th-1 cells.

c) Schematic representation of the main GO terms regulated by HylL6 as inferred from our p-proteomics studies. Red represents downregulated $p$-sites and blue upregulated p-sites upon HylL6 stimulation of human primary Th-1 cells.

\section{Supp. Figure 8:}


a) Venn diagrams comparing the numbers of unique upregulated (left) and downregulated (right) phospho-sites by IL-27 (blue) and HyplL-6 (red) as well as the number of shared phospho-sites.

b) List of most strongly altered phosphosites (downregulated: green; upregulated: red) in response to IL-27 (left) or HyplL-6 (right).

c) GO analysis "cellular location" and "UP keywords" of the phospho-sites regulated by IL27 (red) and HyplL-6 (blue) represented as bubble-plots.

d) Phosphorylation of target proteins related to Treg functions and schematic representation of their activity on T-cells.

\section{Supp. Figure 9:}

a) Kinetics of gene induction in Th-1 cells induced by IL-27 represented as volcano plots. Genes significantly up- or downregulated are highlighted in red ( $p$ value $\leq 0.05$, fold change $\geq+2$ or $\leq-2$ ). Data was obtained from three biological replicates.

b) Kinetics of gene induction in Th-1 cells induced by HyplL-6 represented as volcano plots. Genes significantly up- or downregulated are highlighted in red ( $p$ value $\leq 0.05$, fold change $\geq+2$ or $\leq-2$ ). Data was obtained from three biological replicates.

c) Kinetics of gene induction in Th-1 cells induced by HyplL-6 represented as volcano plots. Genes identified to be significantly up- or downregulated by IL-27 are highlighted in red ( $p$ value $\leq 0.05$, fold change $\geq+2$ or $\leq-2$ ). Data was obtained from three biological replicates.

\section{Supp. Figure 10:}

a) Gene induction kinetics represented as pie-charts, separated for upregulated genes (top panel) and downregulated genes (bottom panel).

b) Kinetics of ISG induction (examples) as heatmap representation comparing IL-27 with HypIL-6 (top) and GSEA reactome pathway enrichment "IFN signaling" for genes induced by IL-27 after $6 \mathrm{~h}$ (bottom). Data represents the mean $\left(\log _{2}\right)$ fold change of three biological replicates.

c) Heatmaps of the top 30 up- and downregulated genes by IL-27 compared to HyplL-6 for $1 \mathrm{~h}, 6 \mathrm{~h}$ and $24 \mathrm{~h}$. Data represents the mean $\left(\log _{2}\right)$ fold change of three biological replicates.

d) Kinetics of IRF1 protein expression as a response to continuous IL-27 and HypIL-6 stimulation in Th-1 cells. Data was obtained from three biological replicates with each two technical replicates, showing mean \pm std dev.

\section{Supp. Figure 11:}

a) Pie charts of proteomic changes (unique \& shared) for upregulated (left) and downregulated (right) proteins in response to IL-27 or HyplL-6 stimulation in Th-1 cells.

b) Left: GSEA reactome pathway enrichment analysis "Interferon signaling" for proteins induced by IL-27. Middle: heatmap representation pathway-associated proteins comparing IL-27 with HyplL-6 stimulation. Data represents the mean $\left(\log _{2}\right)$ fold change of three biological replicates. Right: Localization of the identified proteins in context to the data distribution of IL-27-induced proteomic changes. Pathway-associated proteins are highlighted for IL-27 (blue) and HyplL-6 (red) as well as non-significant data distribution (grey). 
c) Left: GSEA reactome pathway enrichment analysis "Cytokine signaling and immune system" for proteins induced by IL-27. Middle: heatmap representation pathwayassociated proteins comparing IL-27 with HyplL-6 stimulation. Data represents the mean $\left(\log _{2}\right)$ fold change of three biological replicates. Right: Localization of the identified proteins in context to the data distribution of IL-27-induced proteomic changes. Pathway-associated proteins are highlighted for IL-27 (blue) and HyplL-6 (red) as well as non-significant data distribution (grey).

d) Average Intensity distribution of untreated proteomic data. Top up- and downregulated proteins ( $\geq+4 x$ or $\leq-4 x$ change) altered by IL-27 (left) or HyplL-6 (right) stimulation are indicated.

\section{Supp. Figure 12:}

a) Pointwise median and $95 \%$ credible intervals of the WT and chimera mathematical models, using the posterior distributions for the parameters from the ABC-SMC.

b) Dose response curve in RPE1 using the posterior distributions from the ABC-SMC and varying the concentrations of HyplL-6 and IL-27 in the model.

c) Pointwise median and $95 \%$ credible intervals of the WT mathematical model and simulations of a mutant model with $k_{1 b}^{+}=10^{-5} \mathrm{nM}^{-1} \mathrm{~s}^{-1}$ and $k_{1 b}^{-}=10^{0} \mathrm{~s}^{-1}$, using the posterior distributions for the parameters from the ABC-SMC for the other parameters.

\section{Supp. Figure 13:}

a) Fold induction of total STAT1 and STAT3 levels in Th-1 measured by flow cytometry. Data was obtained from two biological replicates.

b) Total levels of STAT1 and STAT3 measured in CD4+ by flow cytometry for healthy control (ctrl) and Lupus patients (SLE). Data was obtained from five (ctrl) and six (SLE) biological replicates. ${ }^{*} P<0.05,{ }^{* *} P \leq 0.01,{ }^{* * *} P \leq 0.001$; n.s., not significant.

c) Ratio of pSTAT1 and pSTAT3 after IL-27 (15min, 2nM) or HyplL-6 (15 min, 10nM) stimulation measured in CD4+ by flow cytometry for healthy control (ctrl) and Lupus patients (SLE). Data was obtained from five (ctrl) and six (SLE) biological replicates normalized to mean ratio of healthy control samples.

d) Tofacitinib titration to inhibit STAT1 and STAT3 phosphorylation by IL-27 (2nM) in Th1 cells (left) and RPE1 cells stably expressing wt IL-27Ra (right). 


\section{Supp. Movie 1:}

Single-molecule co-tracking as a readout for dimerization of cytokine receptors. Cell surface

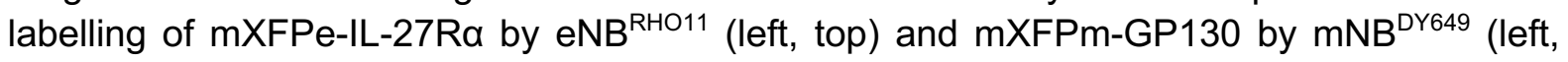
bottom) after stimulation with IL-27 (20nM). In the overlay of the zoomed section of both

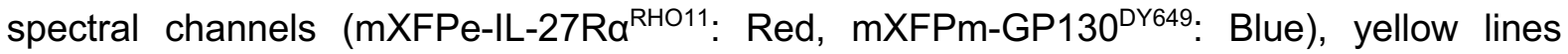
indicate co-locomotion of IL-27Ra and GP130 ( $\geq 10$ steps). Acquisition frame rate: $30 \mathrm{~Hz}$, Playback: real time.

\section{Supp. Movie 2:}

Dynamics of IL-27-induced receptor assembly. Formation of a single-molecule heterodimer of mXFPe-IL-27Ra ${ }^{\mathrm{RHO} 11}$ (Red) and mXFPm-GP130 ${ }^{\mathrm{DY} 649}$ (Blue) in presence of IL-27. Yellow lines indicate co-locomotion of IL-27Ra and GP130 ( $\geq 10$ steps). Acquisition frame rate: $30 \mathrm{~Hz}$, Playback: real time with break at time of receptor dimerization.

\section{Supp. Movie 3:}

Ligand-induced heterodimerization of IL-27Ra and GP130. Overlay of the two spectral

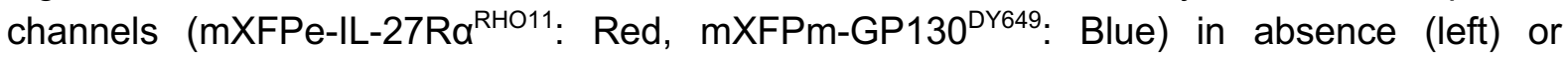
presence (right) of IL-27 (20nM). Yellow lines indicate co-locomotion of IL-27Ra and GP130 ( $\geq 10$ steps). Acquisition frame rate: $30 \mathrm{~Hz}$, Playback: real time.

\section{Supp. Movie 4:}

Ligand-induced homodimerization of GP130. Overlay of the two spectral channels (mXFPmGP130 RHO11: Red, mXFPm-GP130 ${ }^{\text {DY649: }}$ Blue) in absence (left) or presence (right) of HyplL-6 (20nM). Yellow lines indicate co-locomotion of IL-27Ra and GP130 ( $\geq 10$ steps). Acquisition frame rate: $30 \mathrm{~Hz}$, Playback: real time. 
Fig. 1

a) $\overbrace{\mathrm{P} 28 Q_{\mathrm{EBI} \mid 3}}^{\mathrm{IL}-27}$

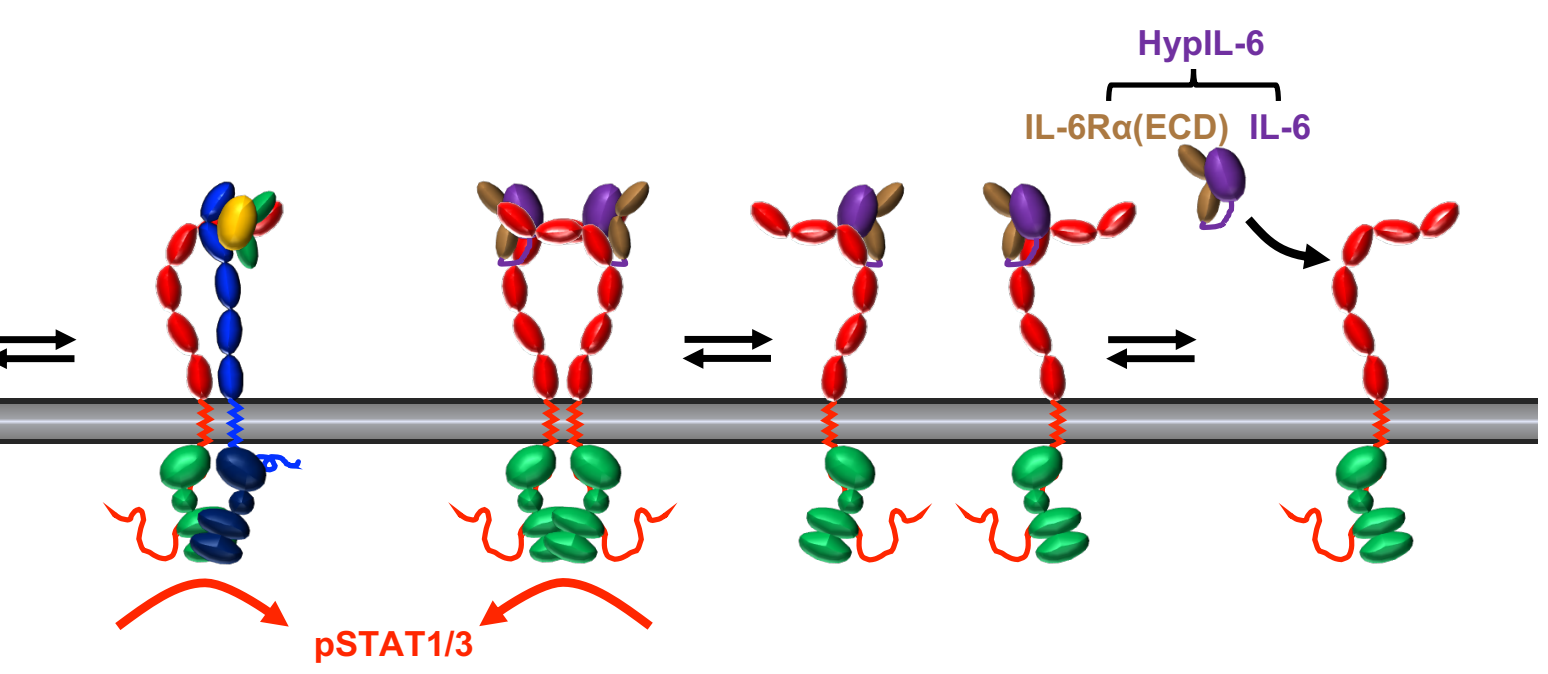

b)

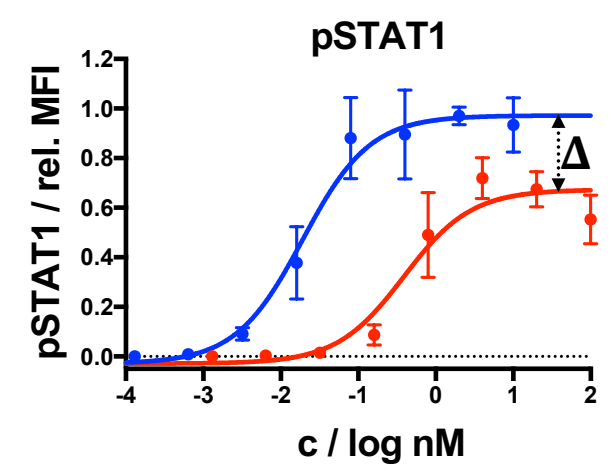

d)

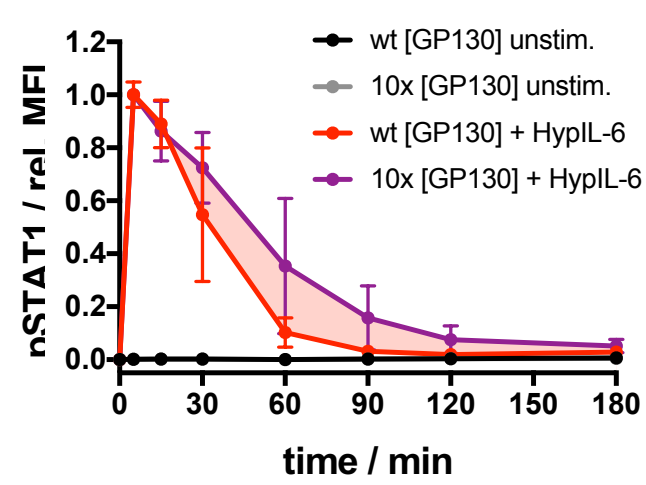

e)

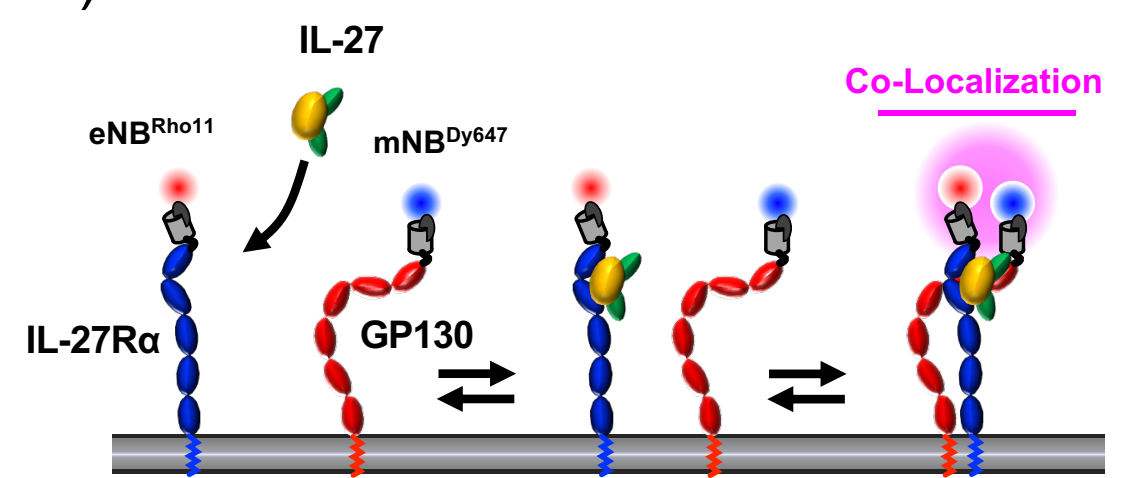

g)

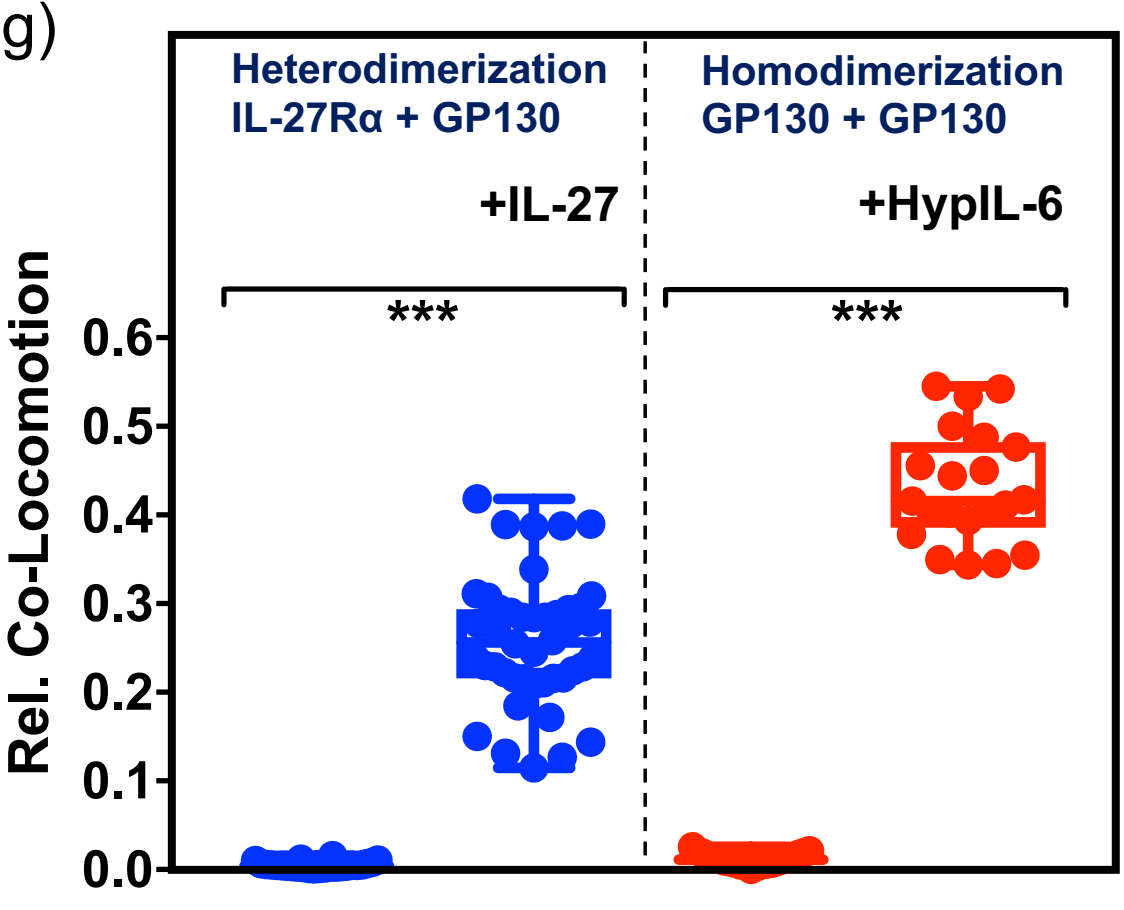

c)
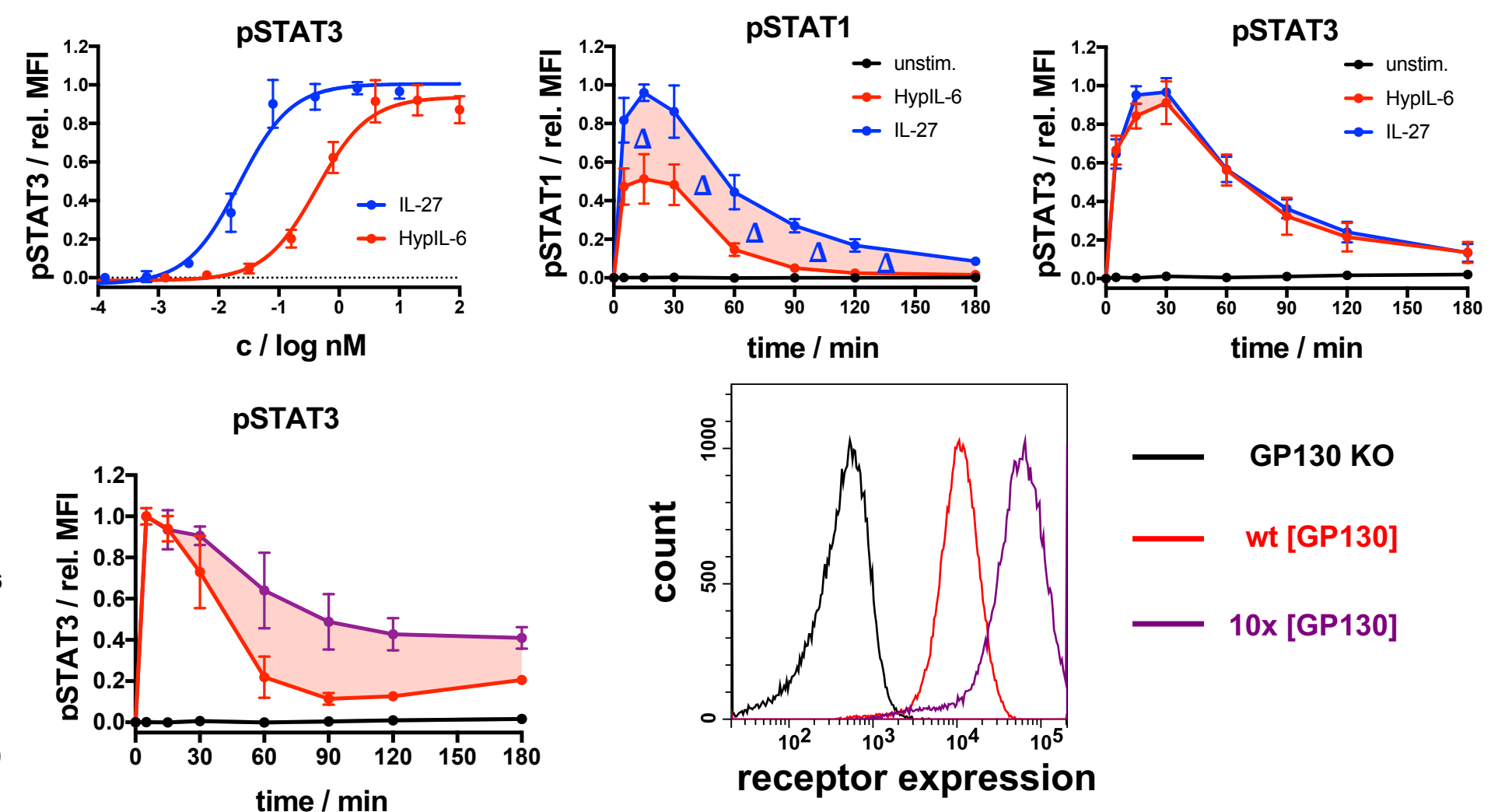

f)

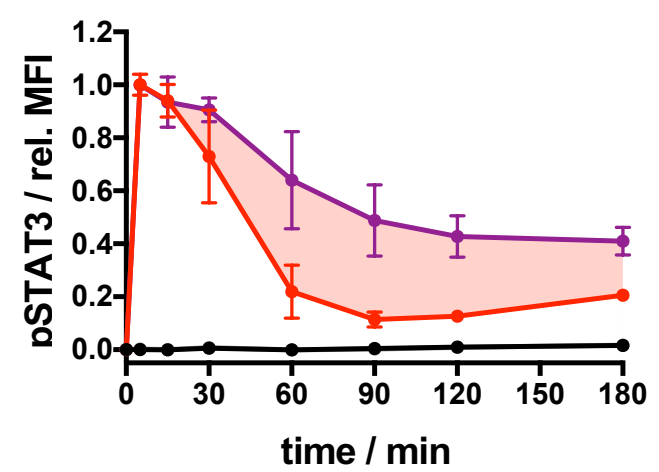

IL-27Ra
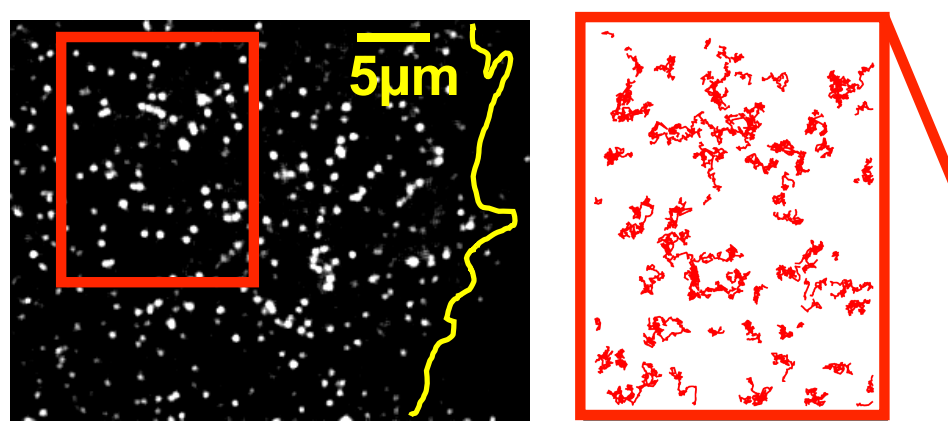

IL-27Ra

GP130

Dimers

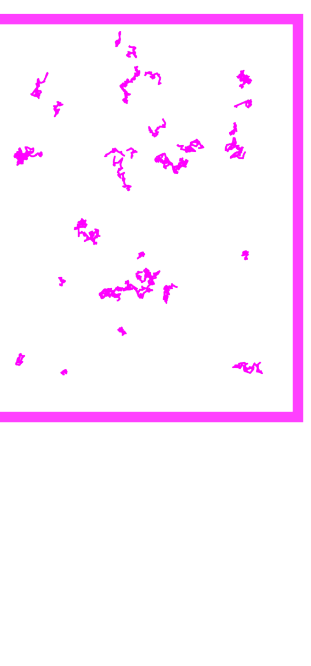

h)
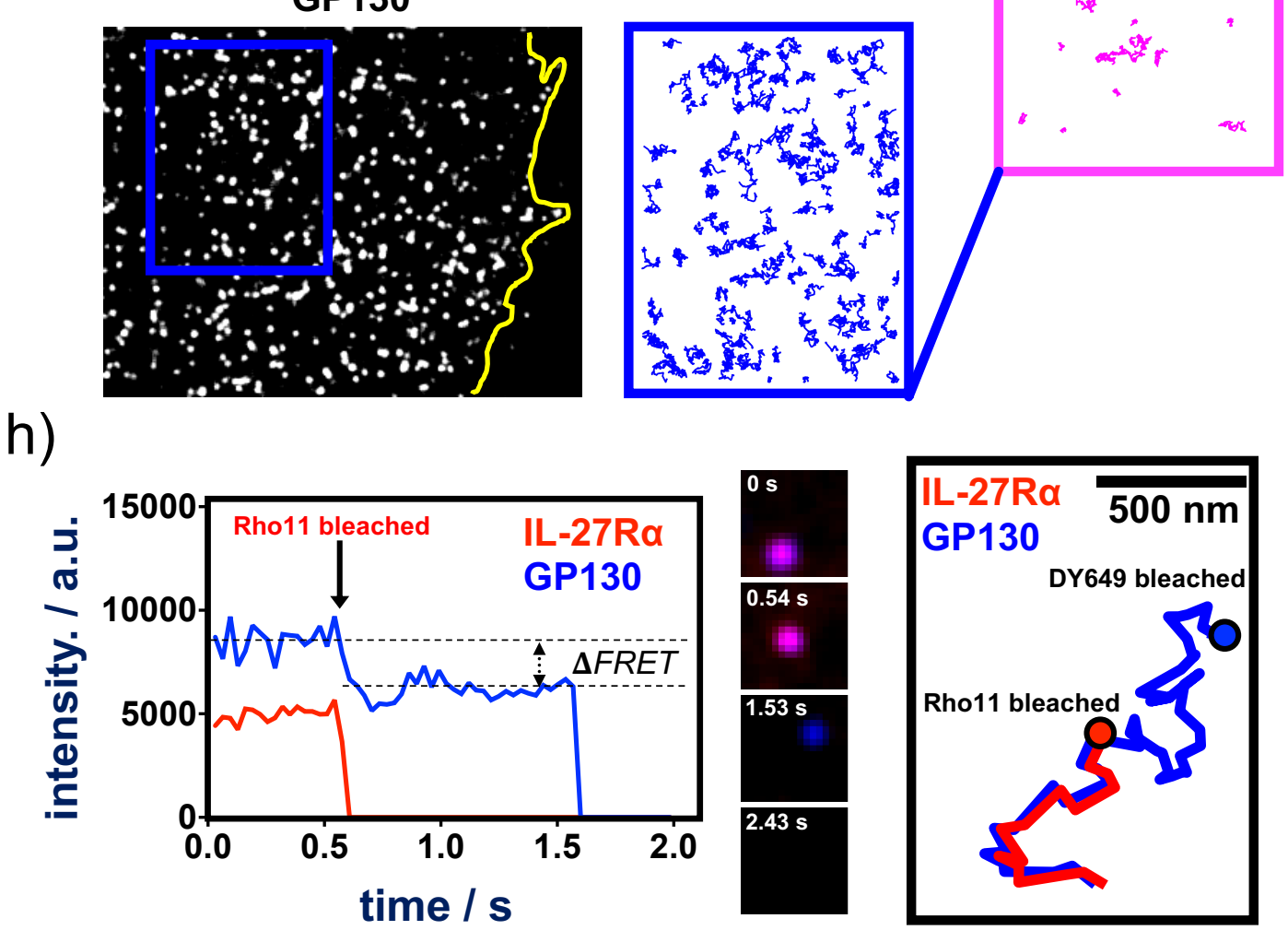
Fig. 2

a)

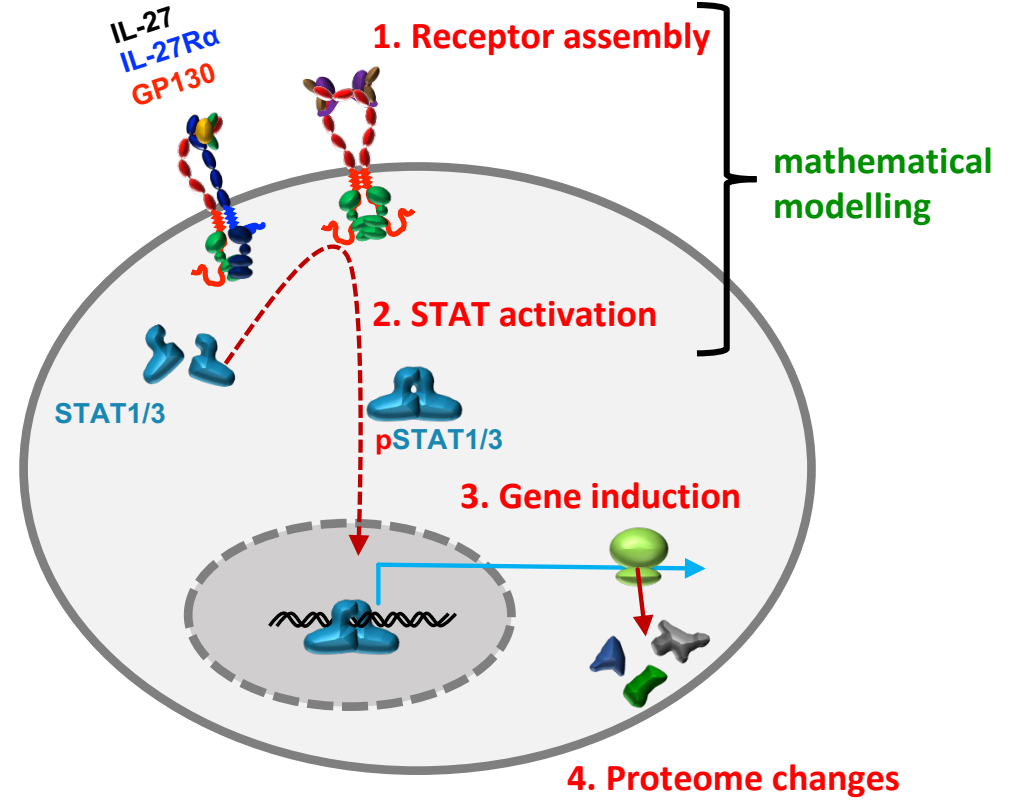

b)

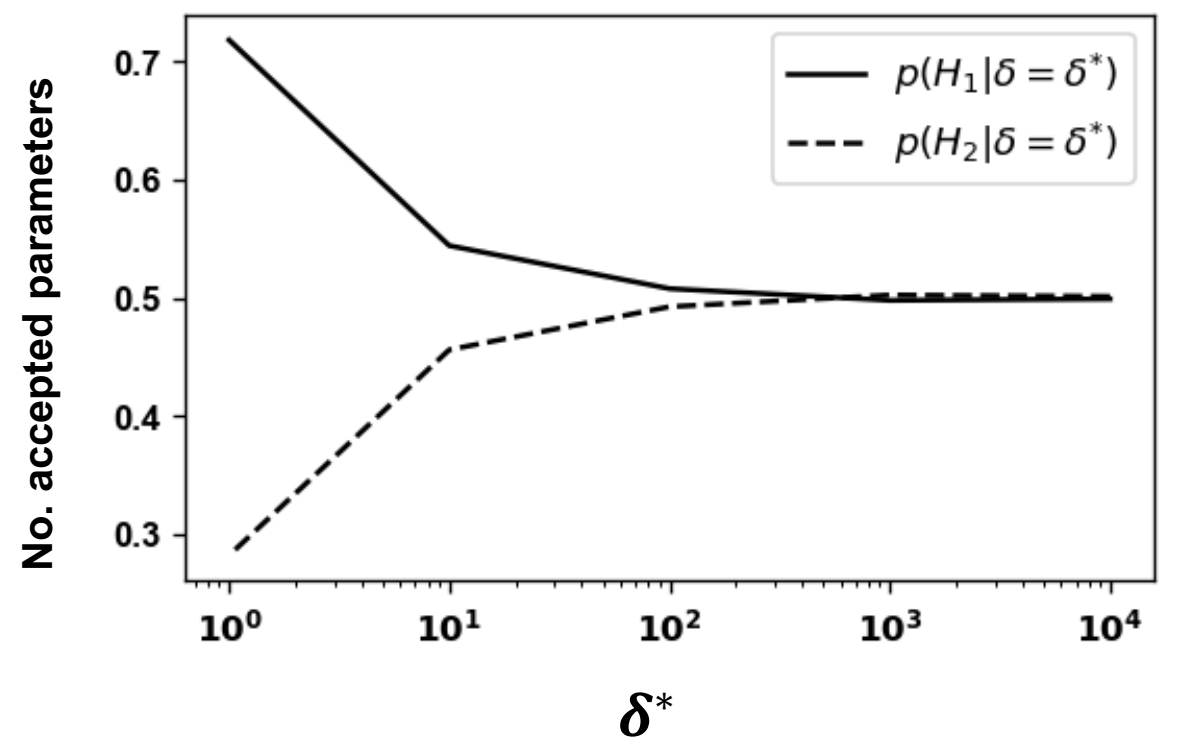

RPE1

Th-1

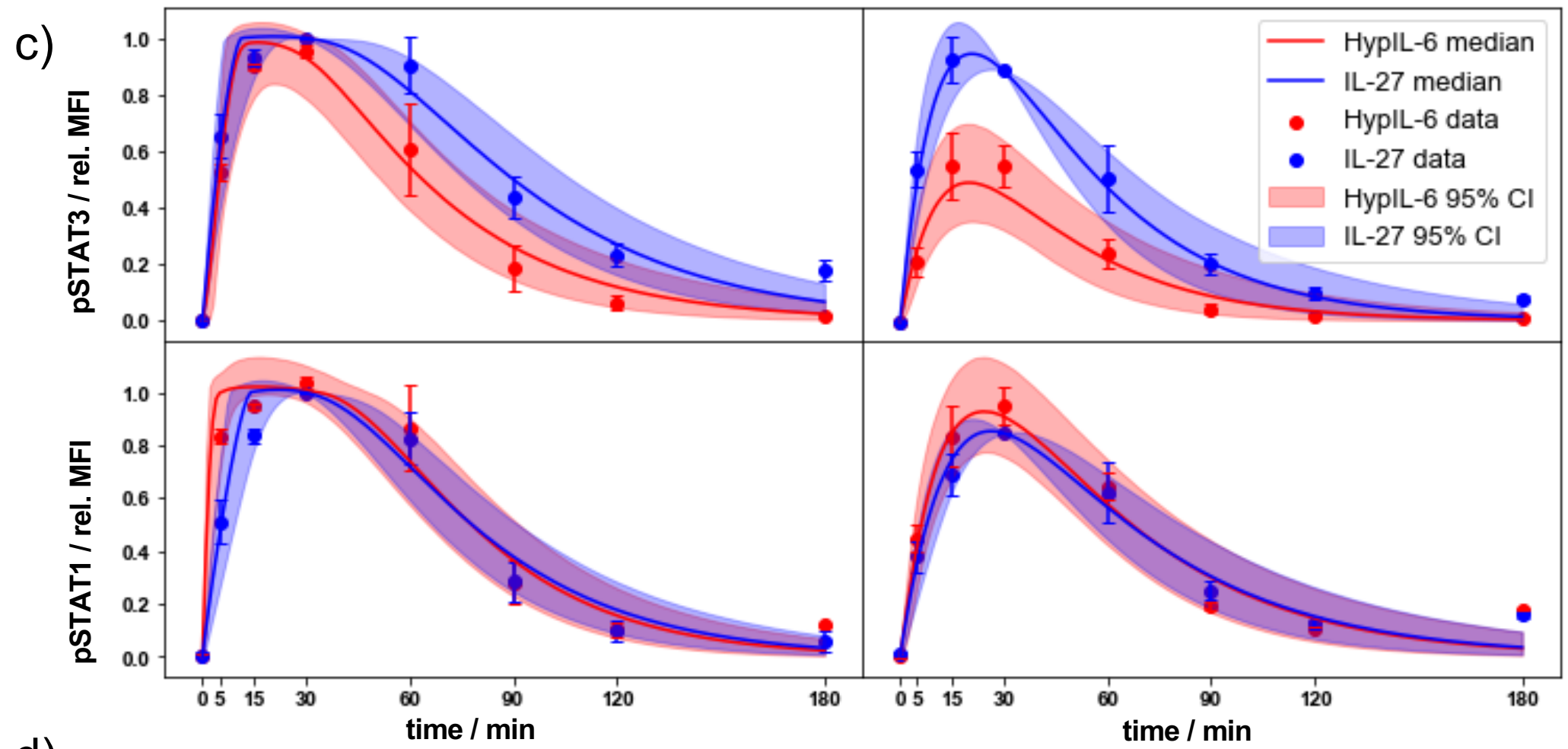

d)
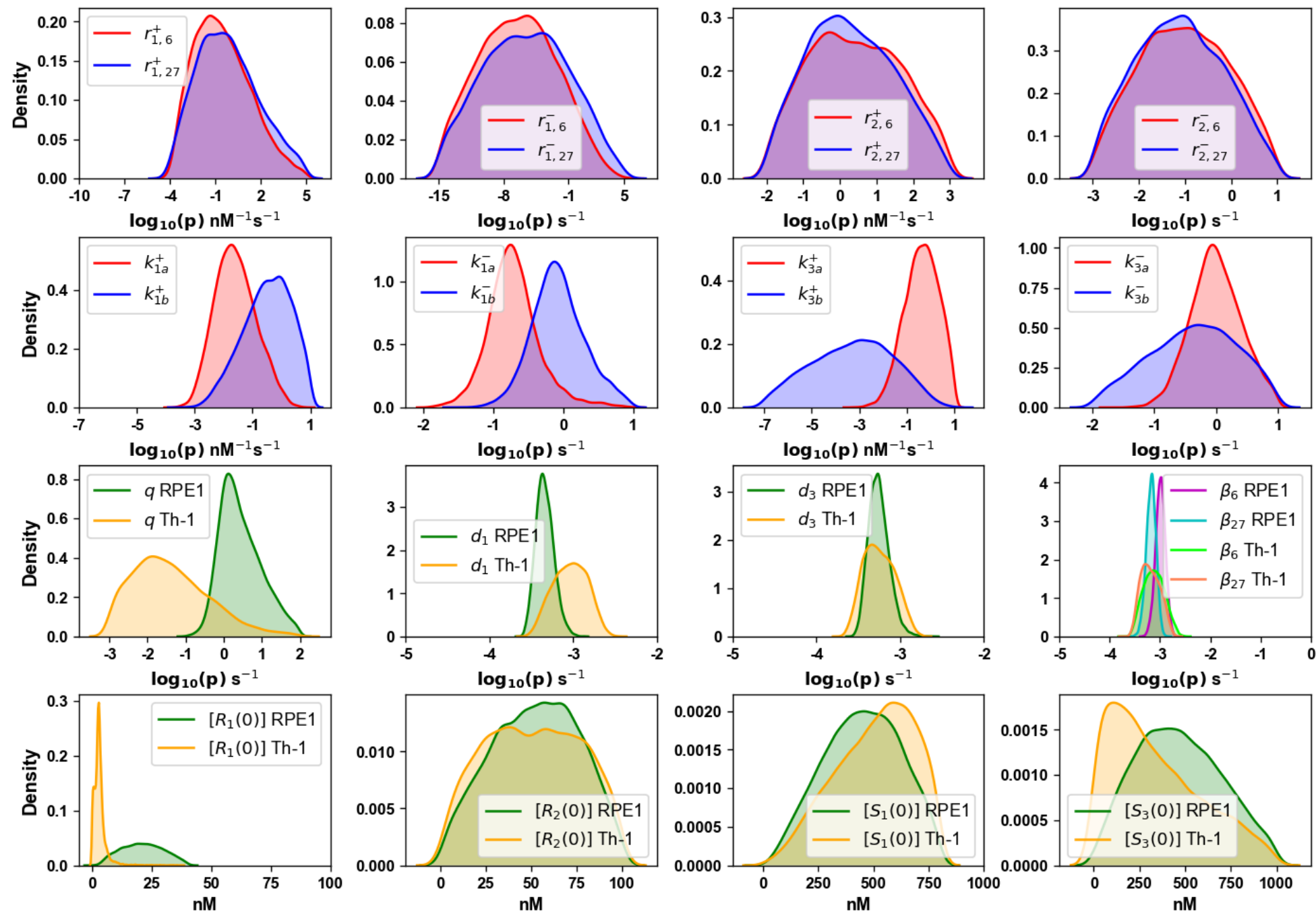
Fig. 3

a) Y543

IL-27Ra cytoplasmic domain

TSGRCYHLRHKVLPRWVWEKVPDPANSS S GQPHMEQVPEAQPLGDLPI LEVEEMEPP PVMES S QPAQATAPLDSGYEKHFLPTPEELGLLGPPRPQVLA*

b) Y613
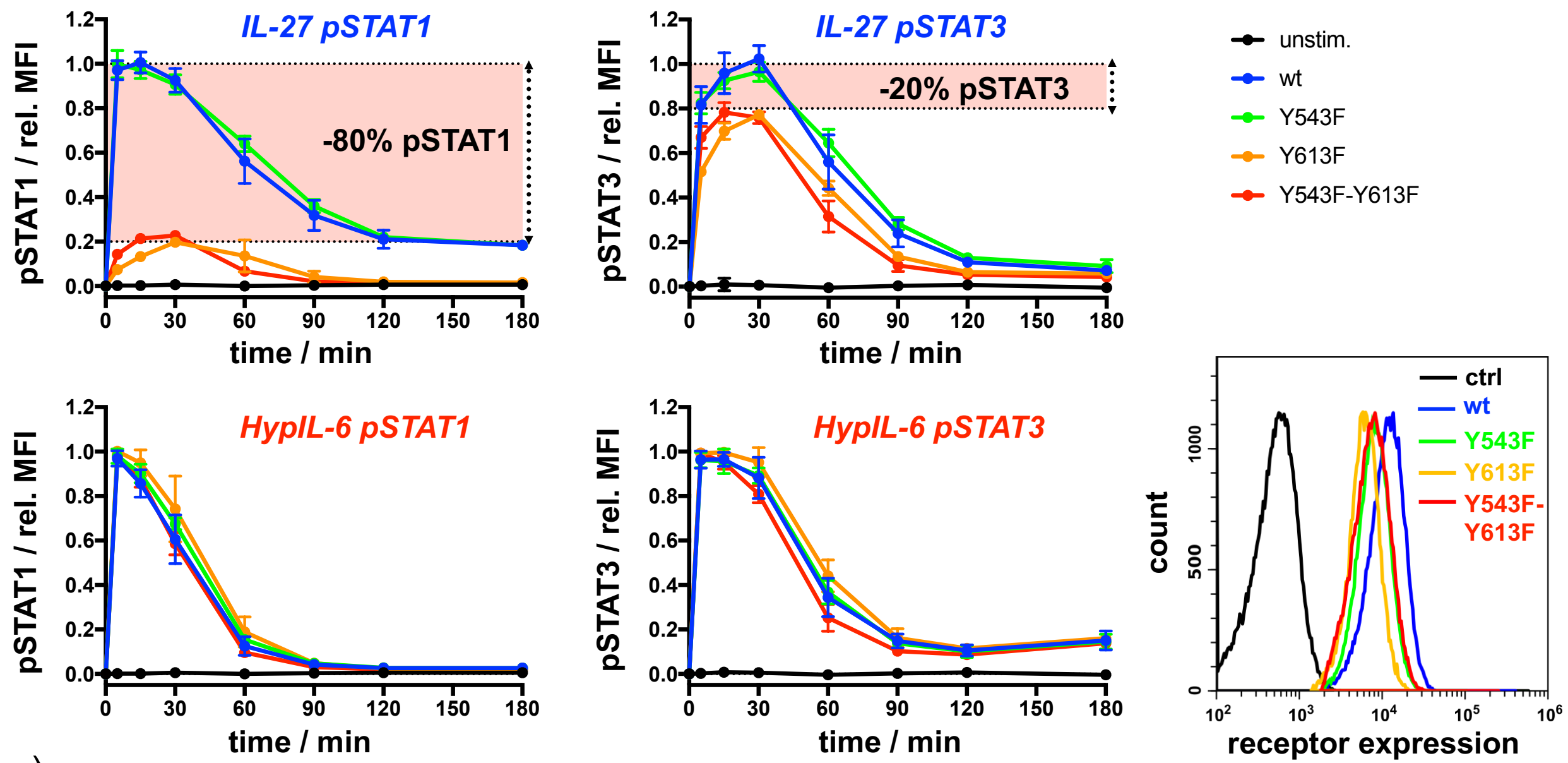

c)

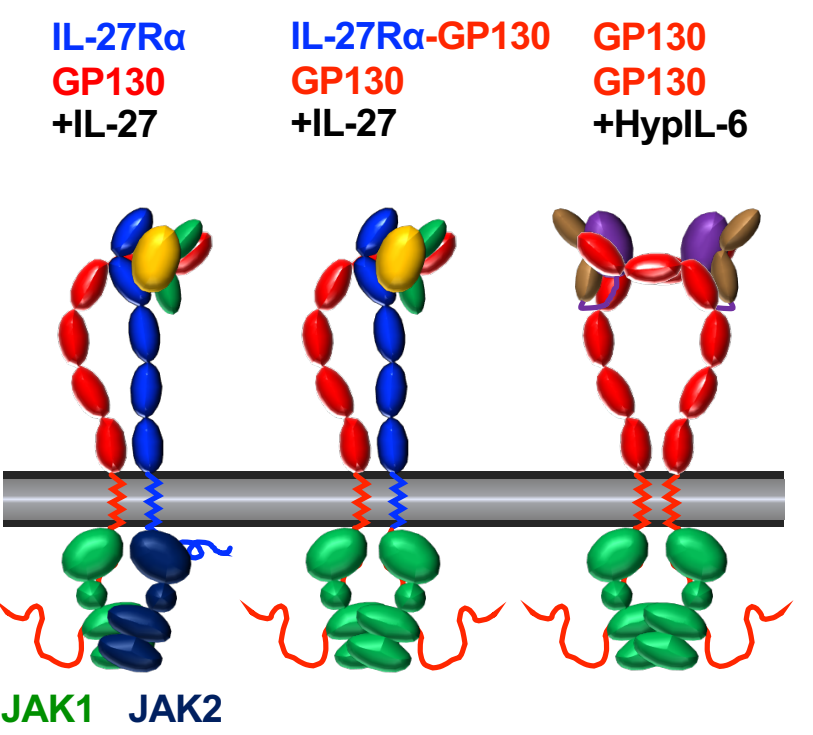

d)
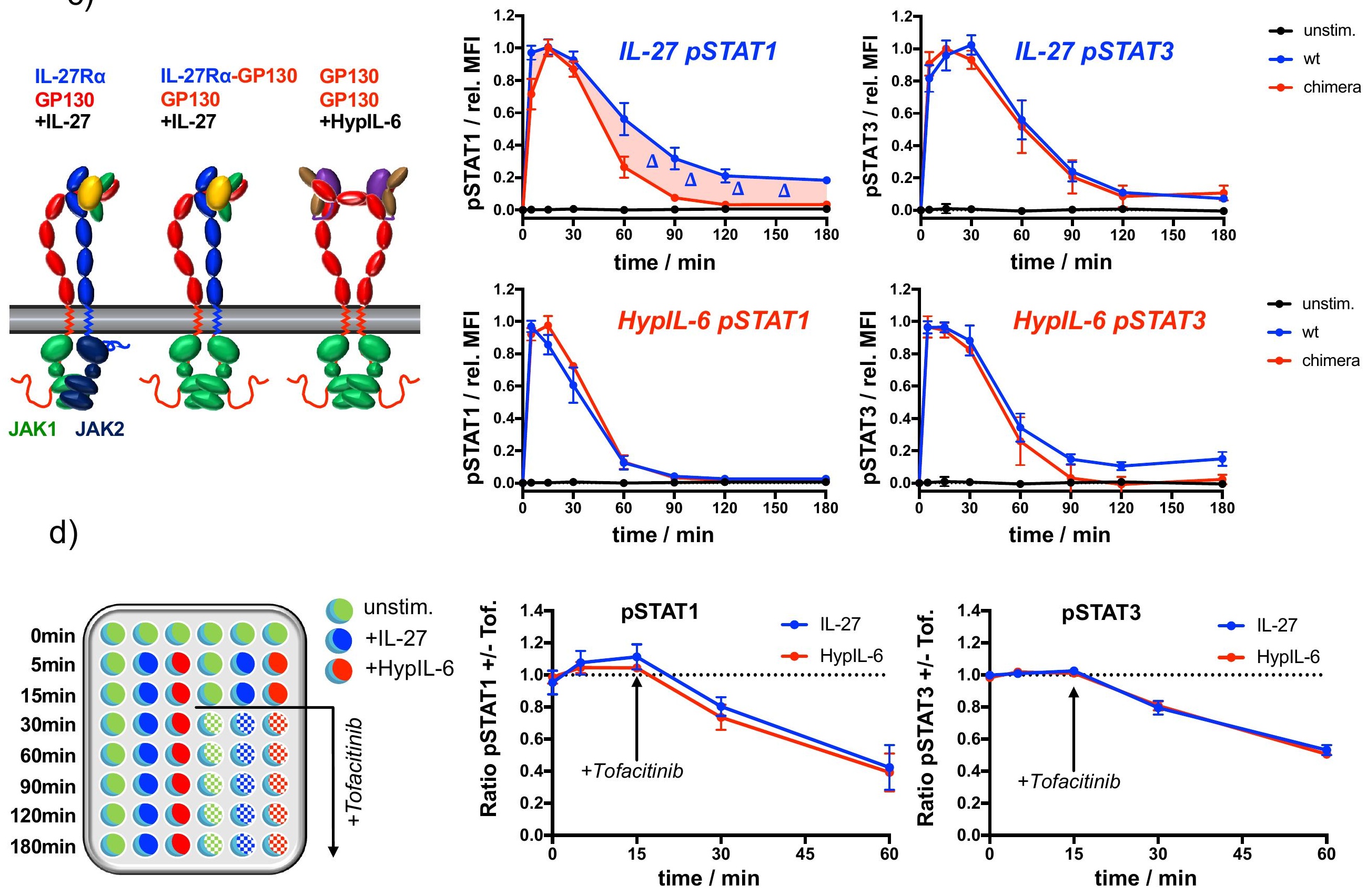
Fig. 4

a)

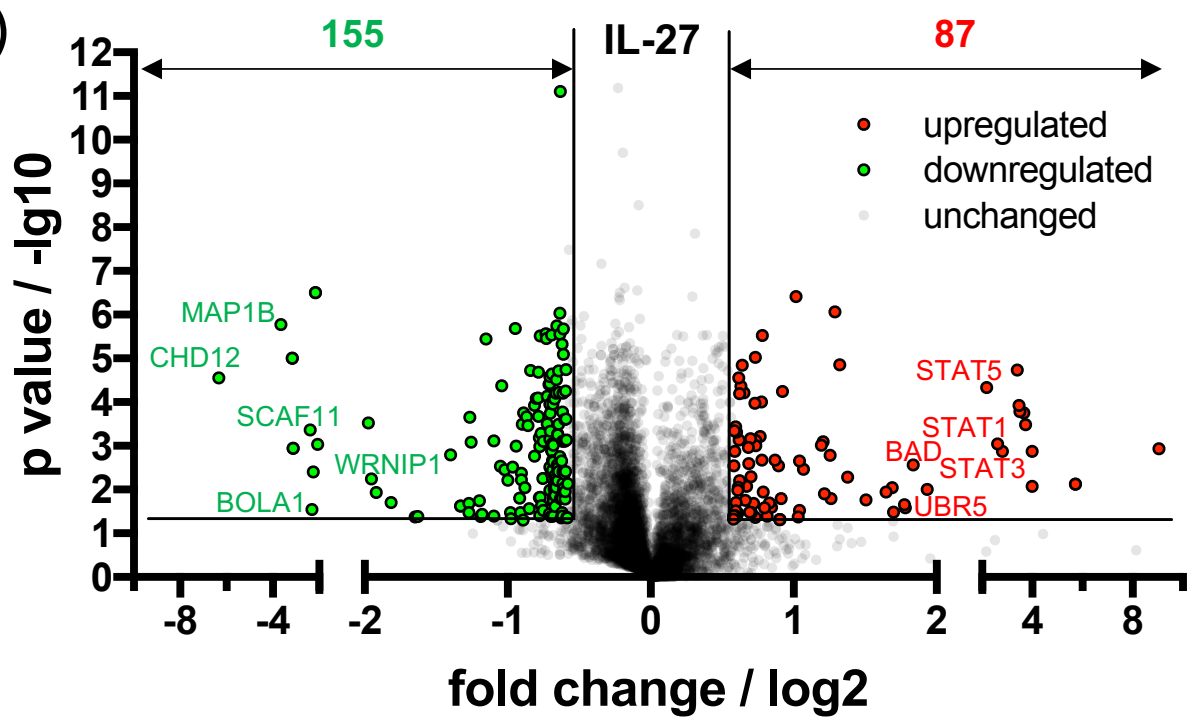

b) shared and differentially regulated p-sites
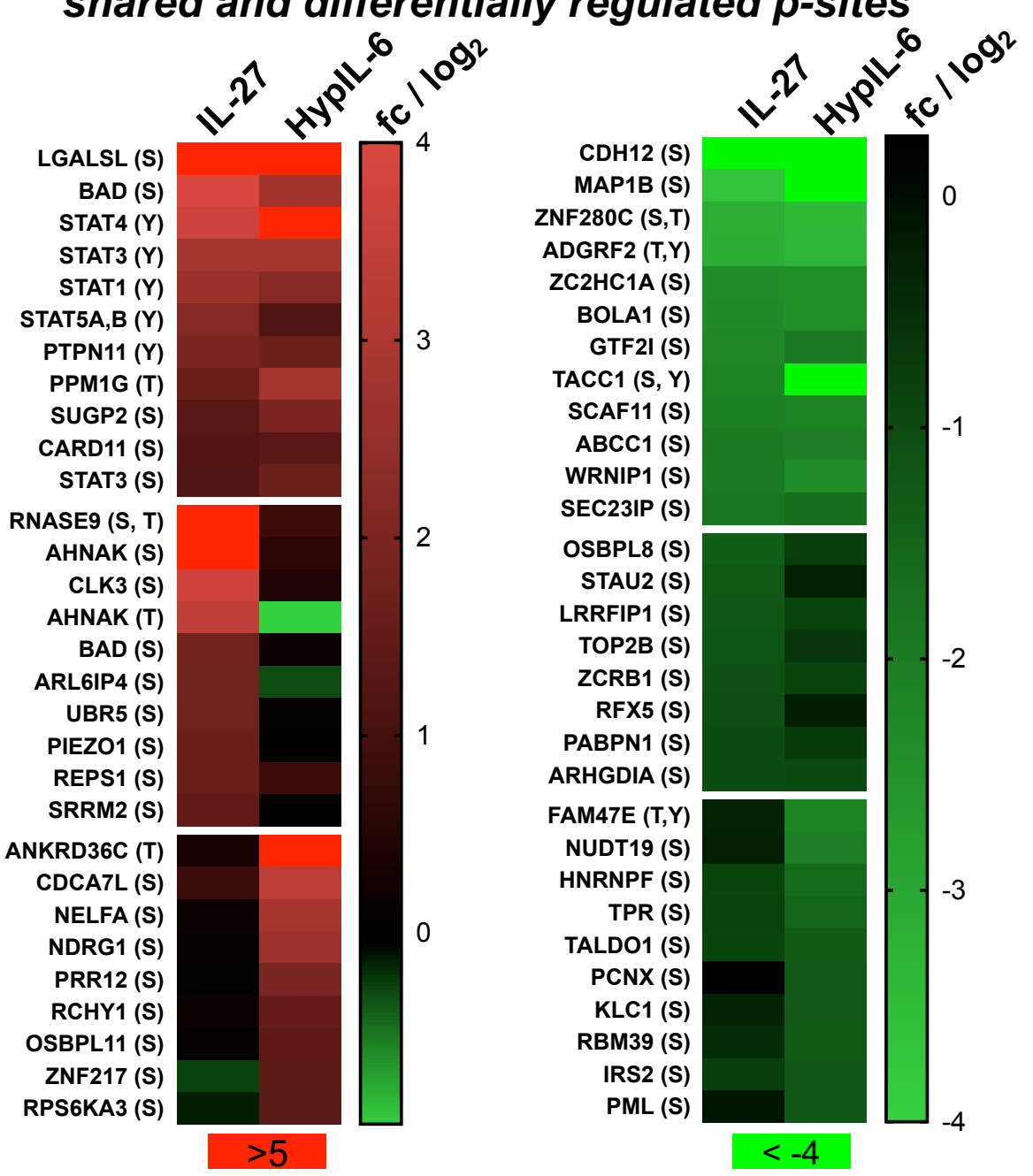

e)

f)

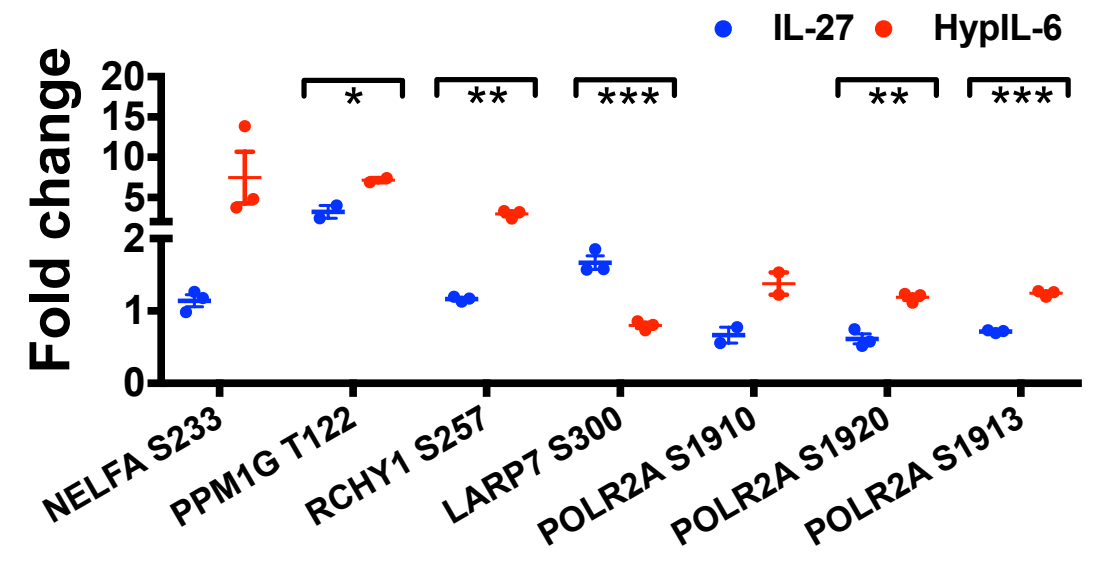

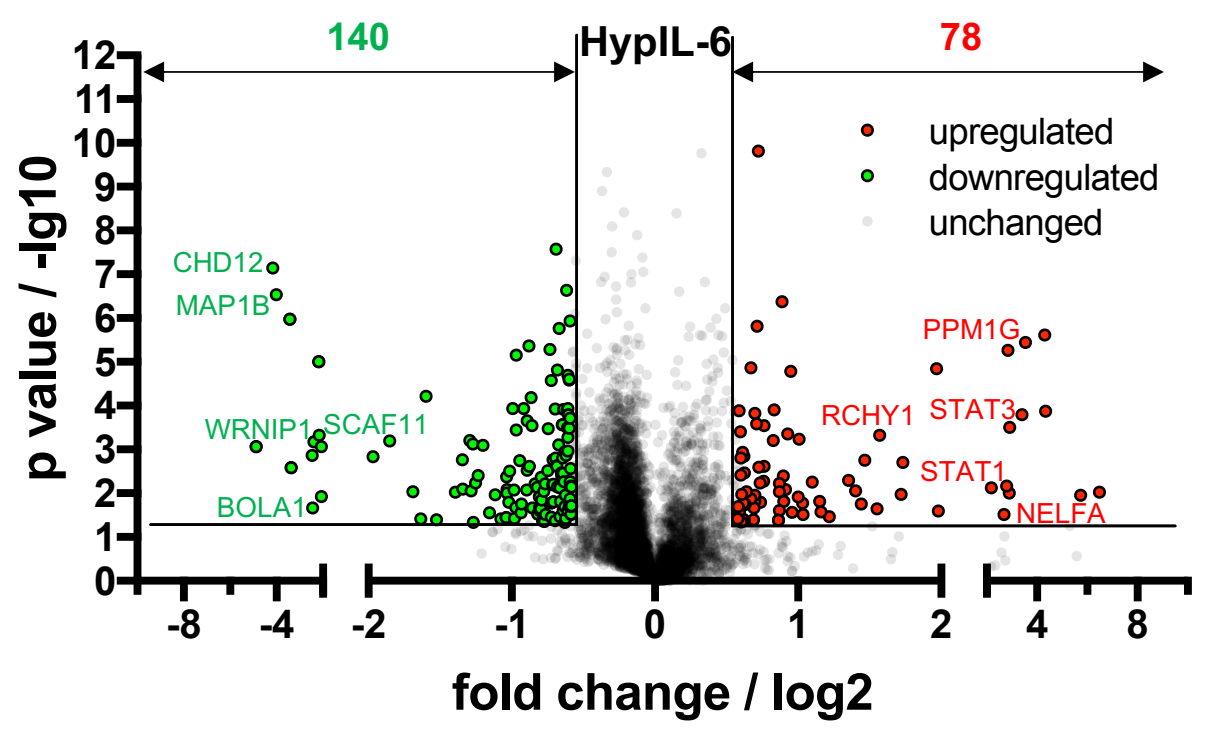

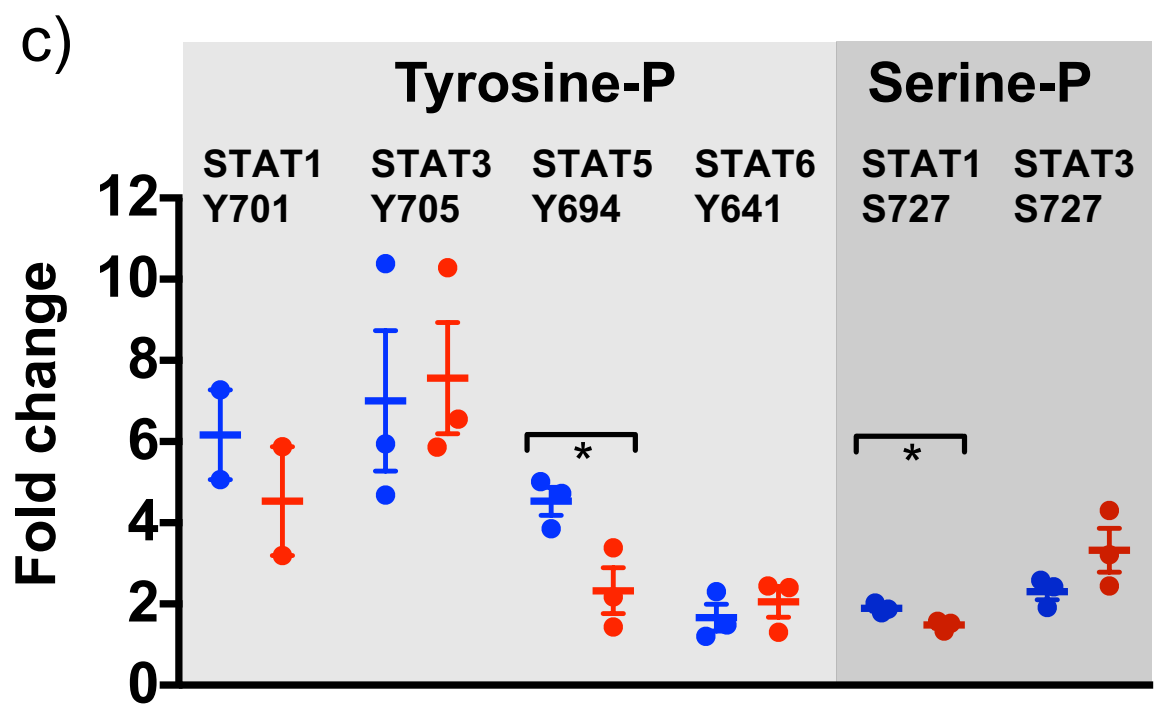

- IL-27 • HyplL-6

d)
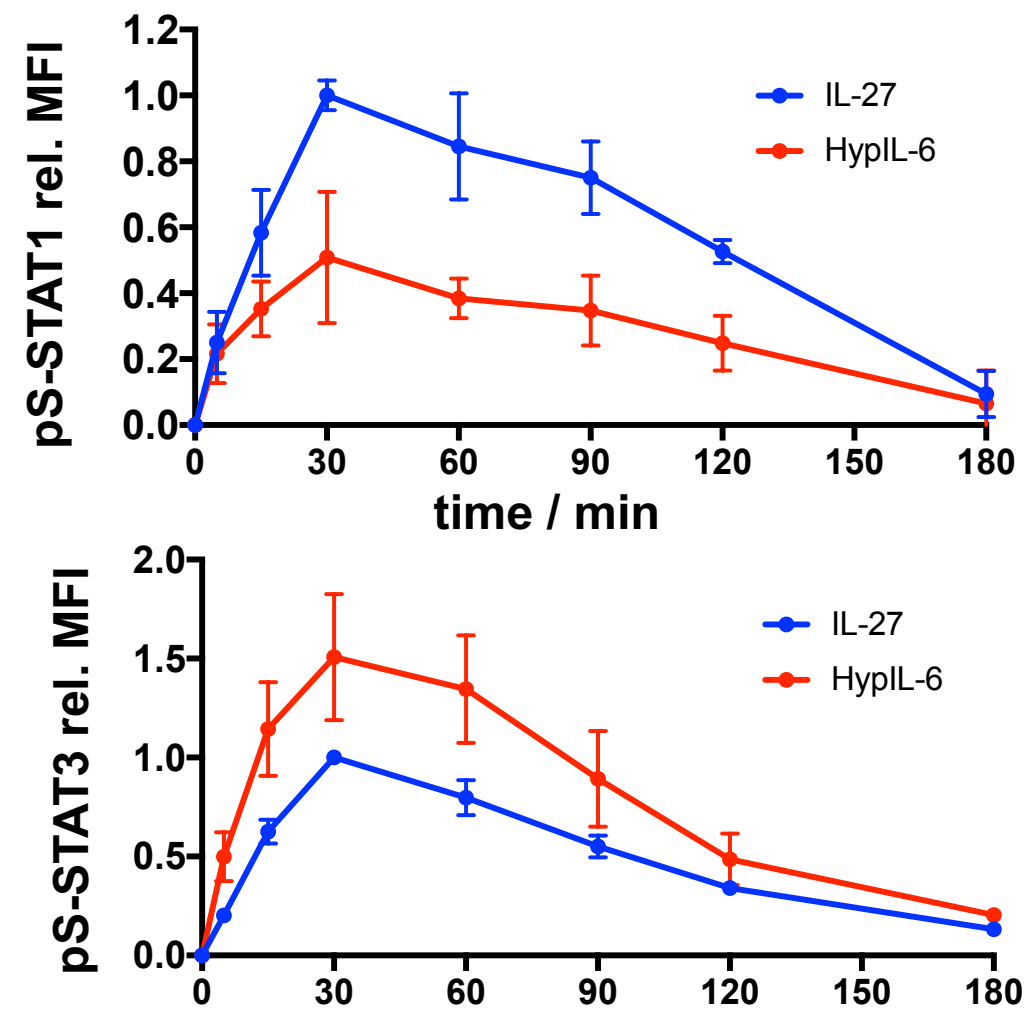

time / $\mathrm{min}$

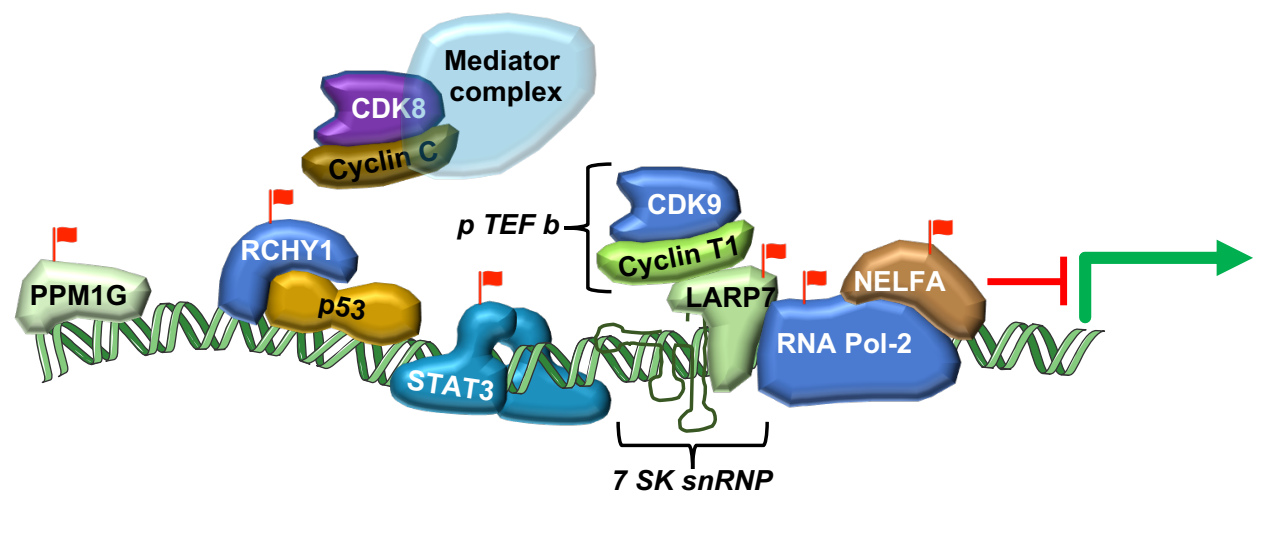


Fig. 5

a)

b)
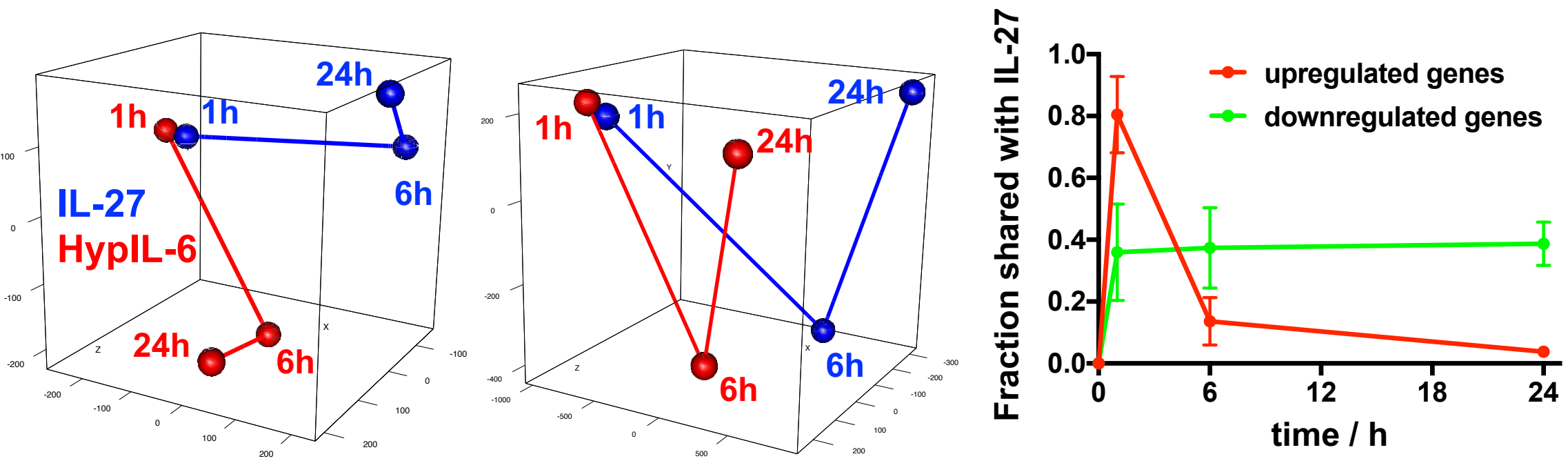

c) upregulated

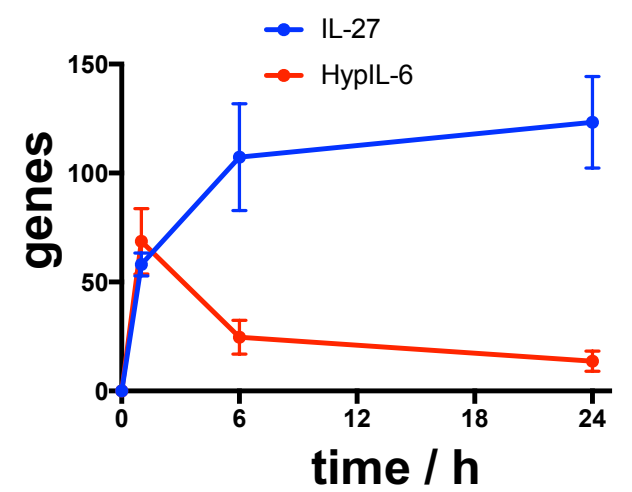

e)

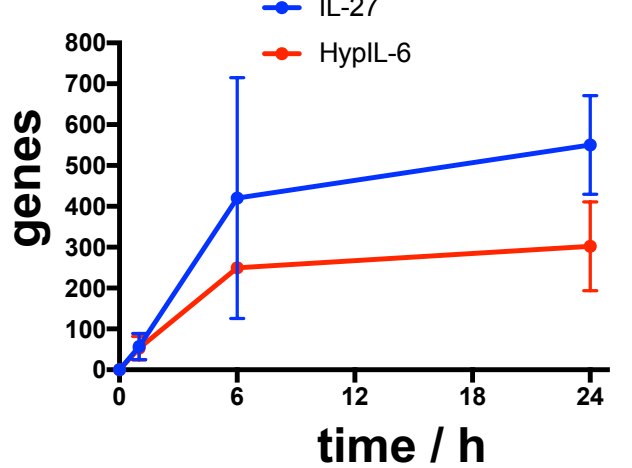

d)

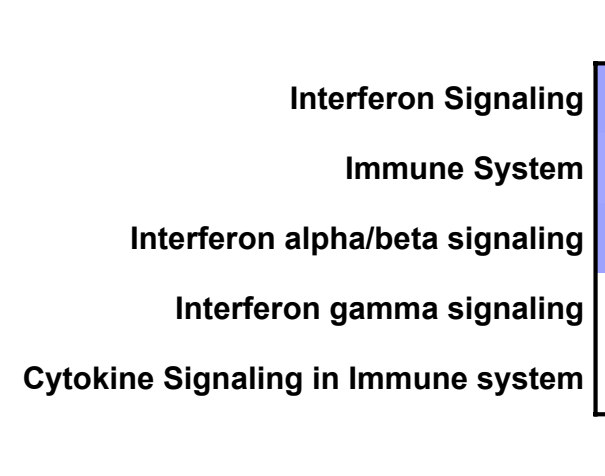

IL-27

HyplL-6

1h 6 h $24 \mathrm{~h} 1 \mathrm{~h} 6 \mathrm{~h} 24 \mathrm{~h}$ fc / log2

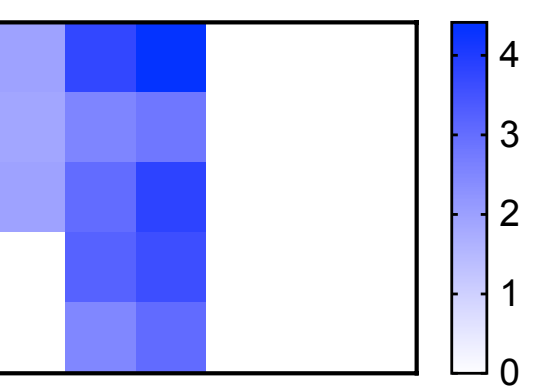

\section{1h $6 \mathrm{~h} 24 \mathrm{~h} 1 \mathrm{~h} 6 \mathrm{~h} 24 \mathrm{~h}$}
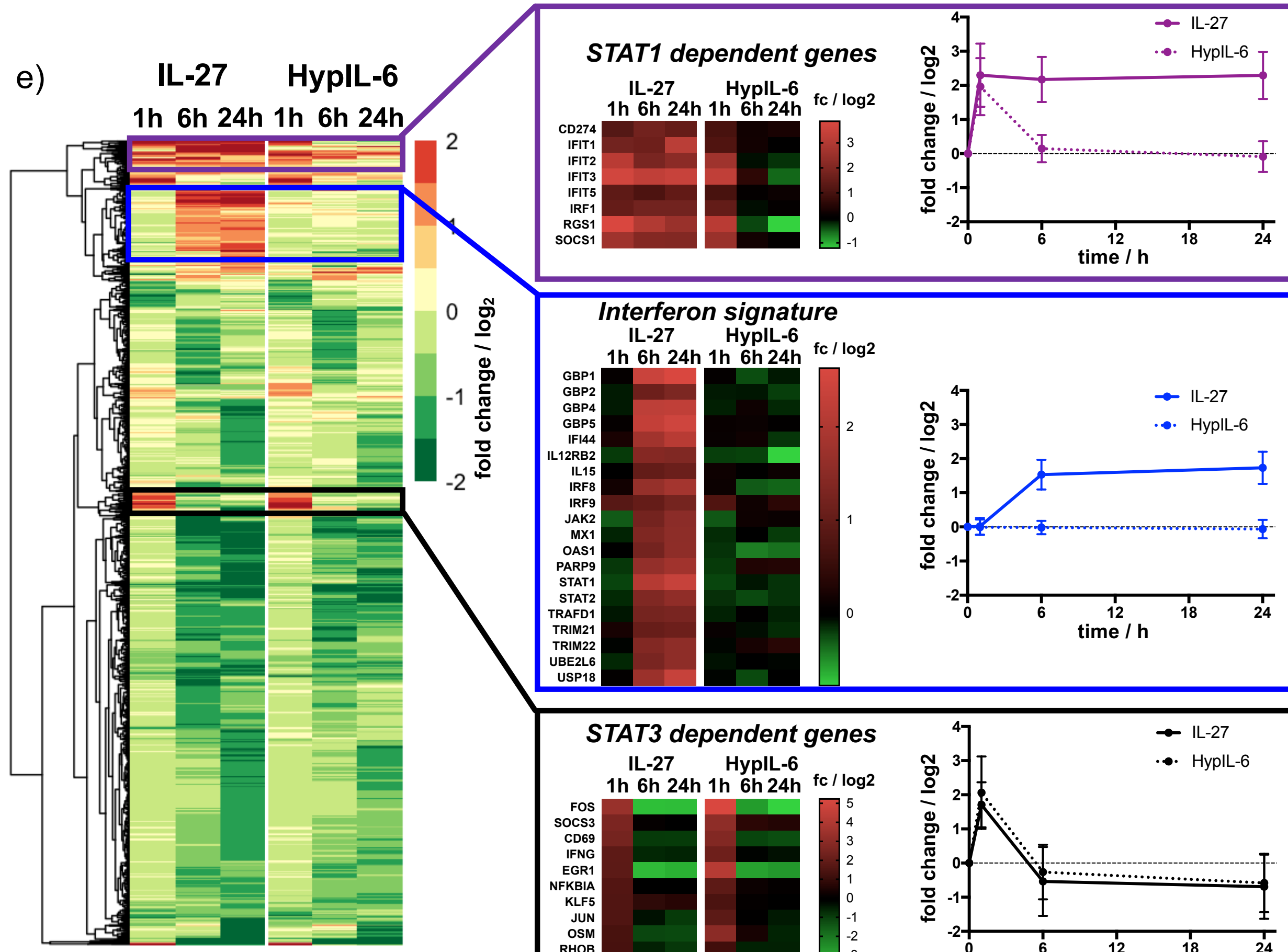

STAT3 dependent genes
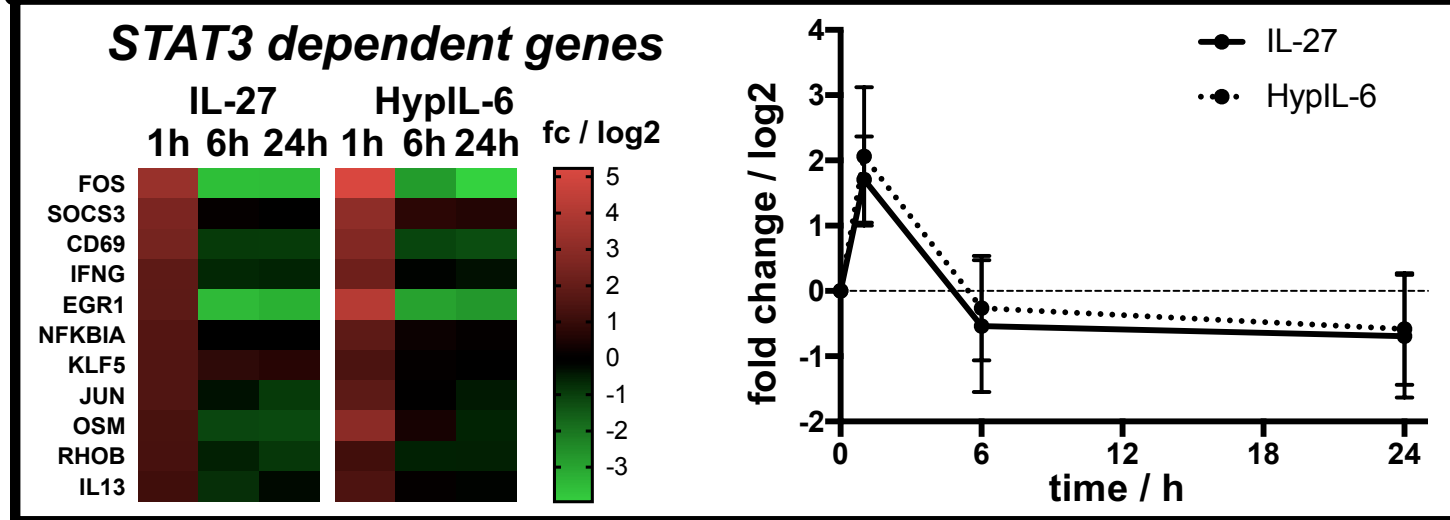
Fig. 6

a)

IRF1 protein levels

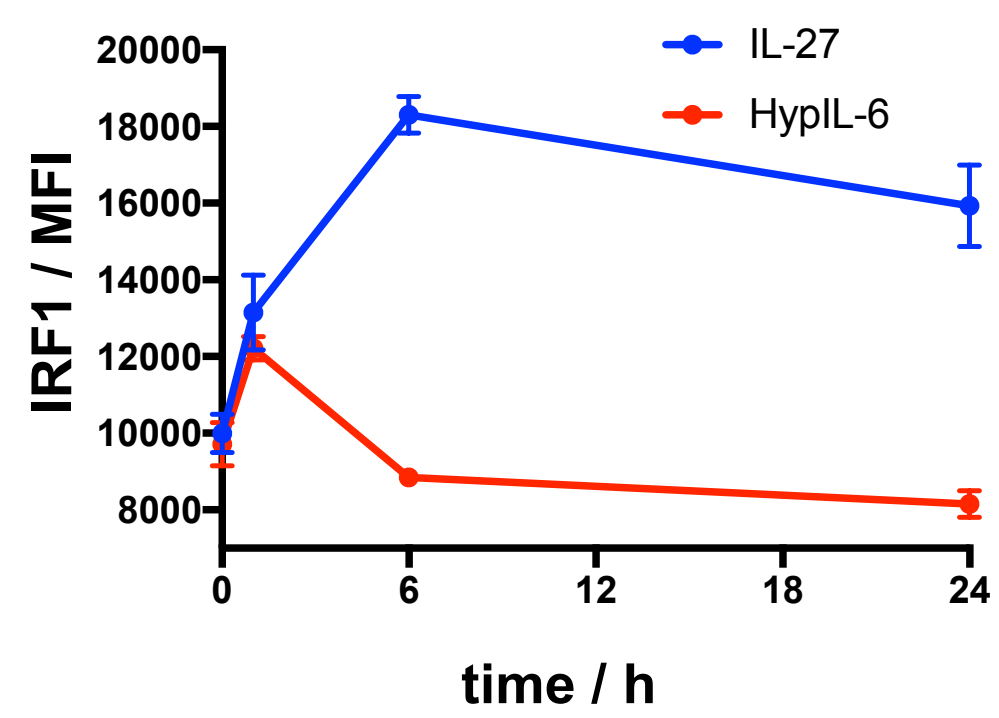

c)
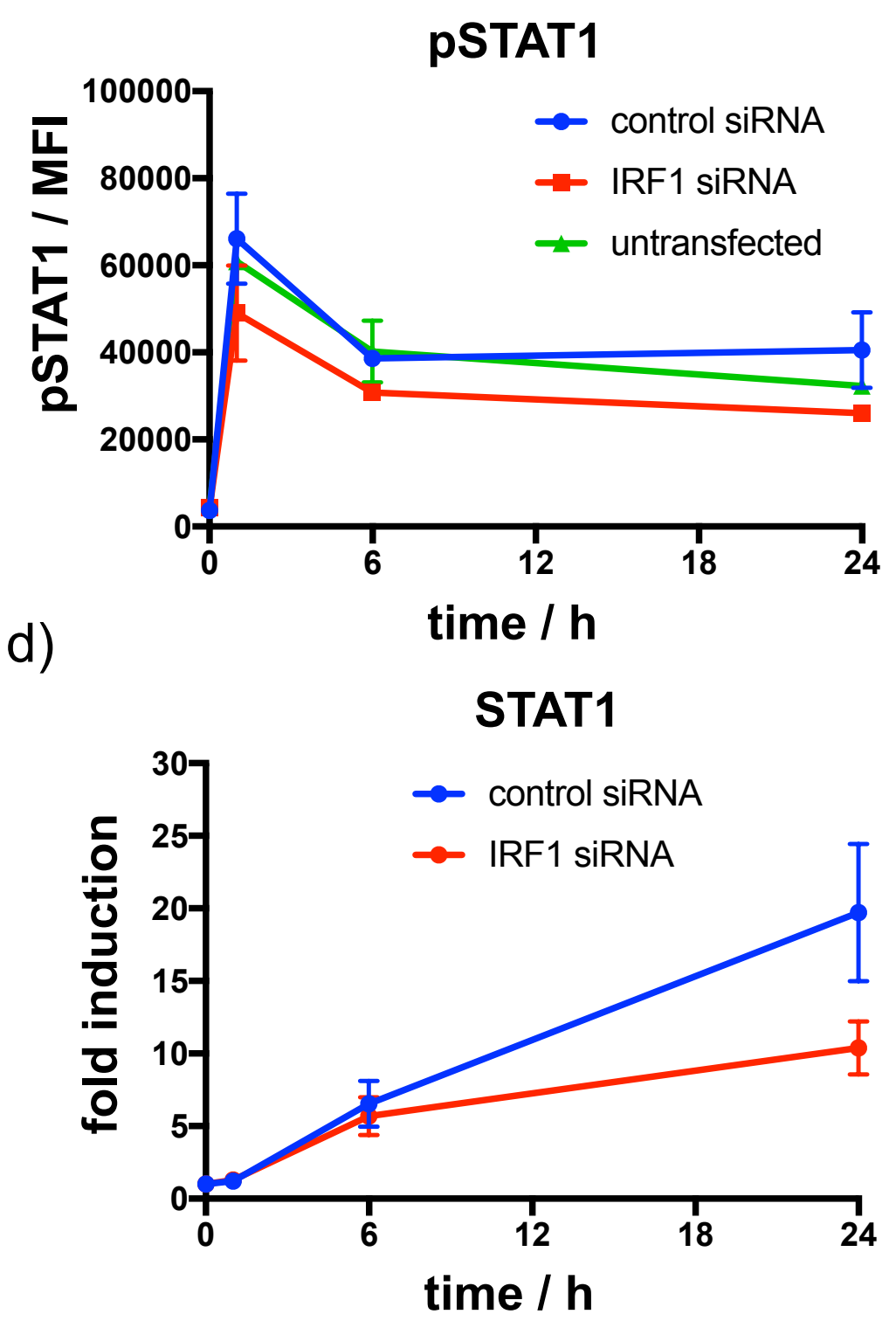

OAS1

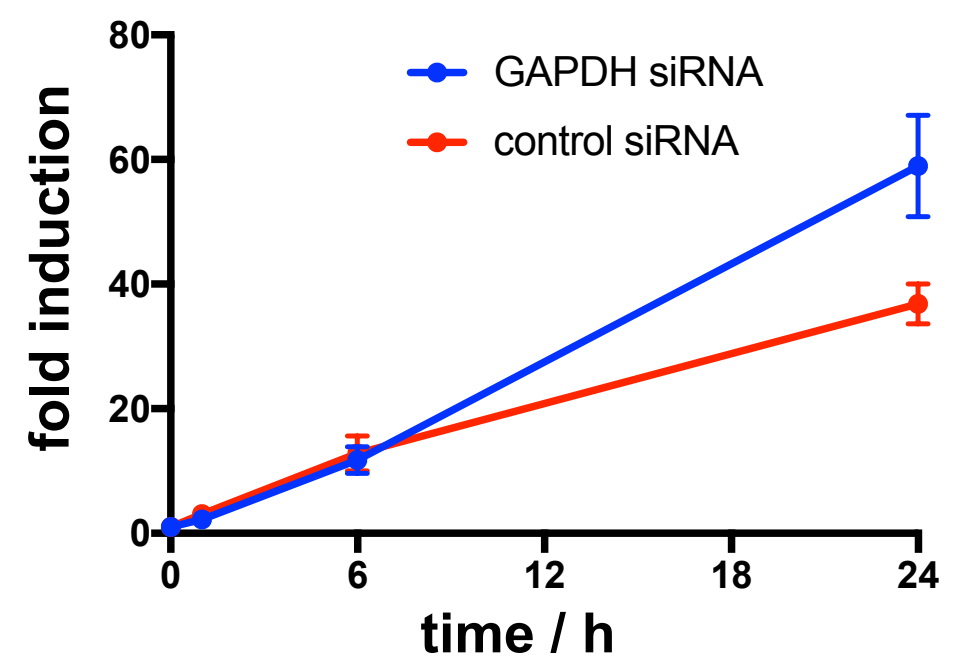

b) IRF1 protein levels

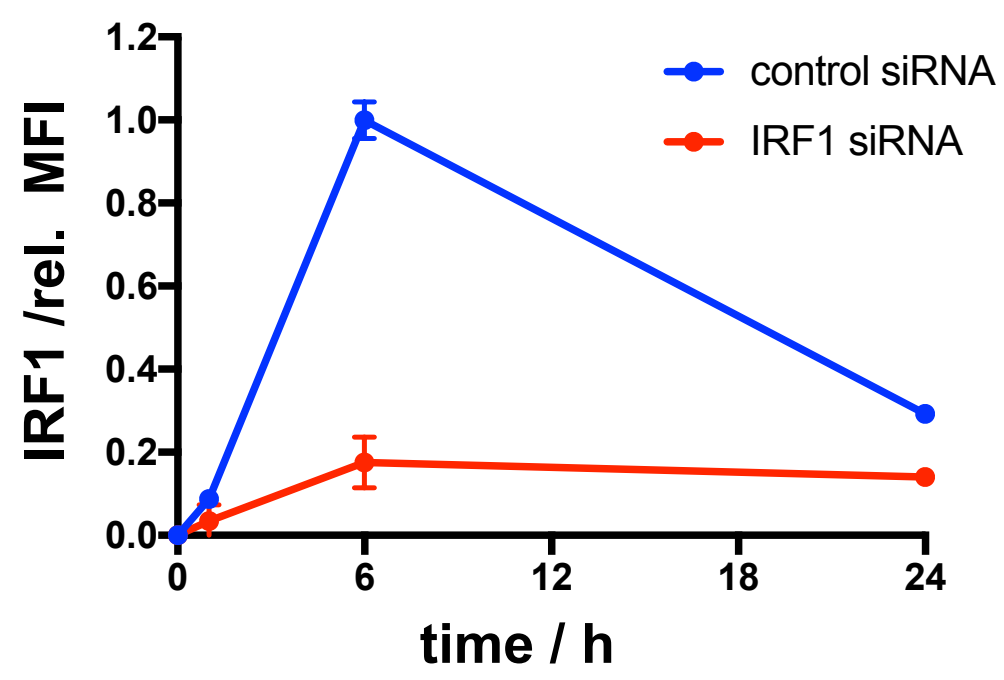

PSTAT3
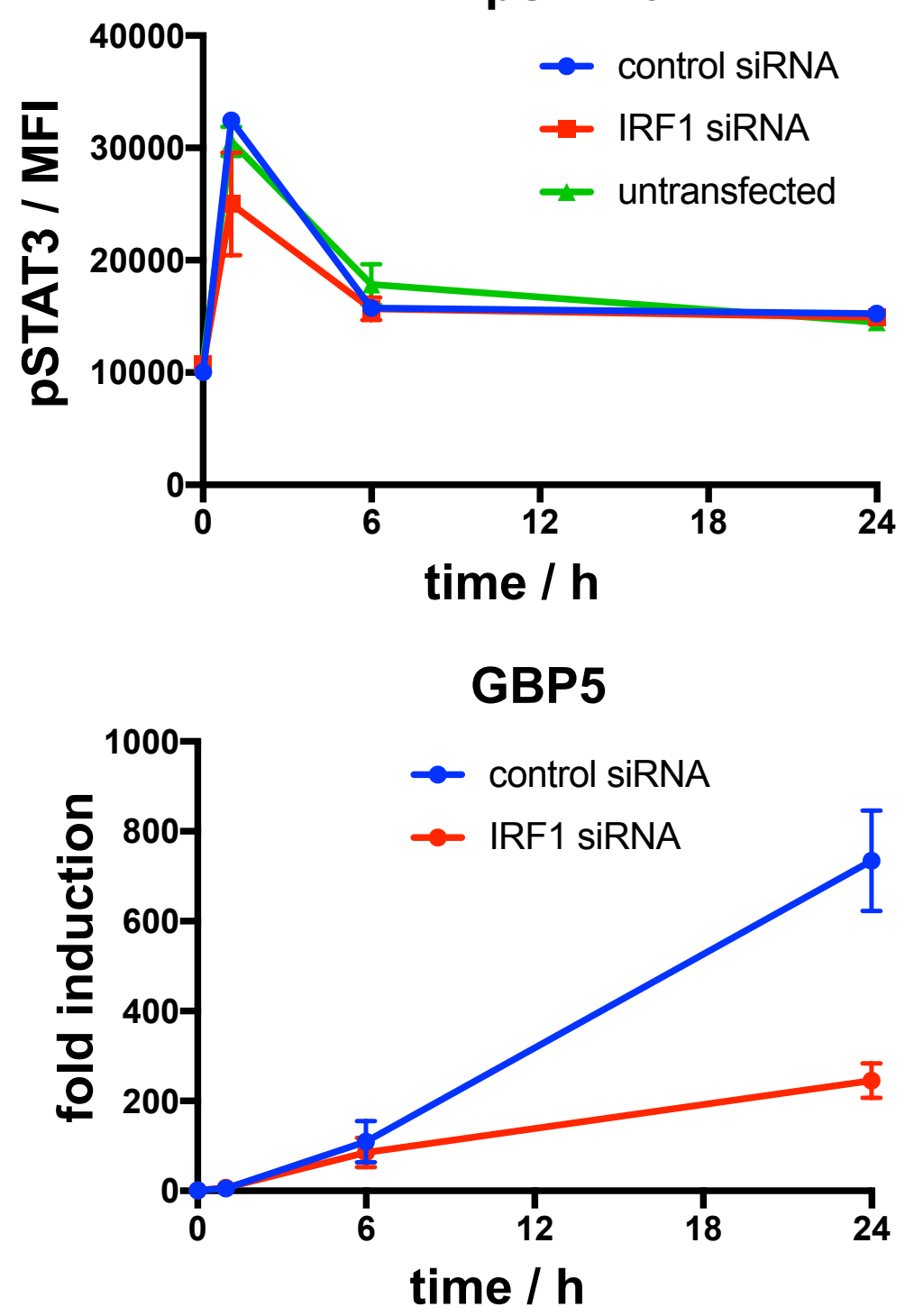

socs3

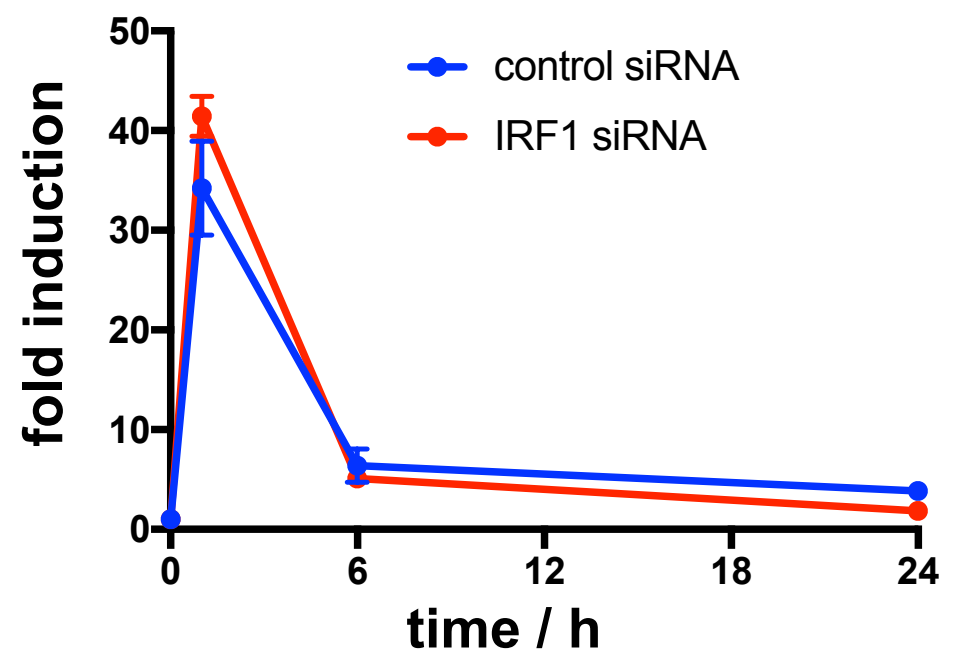


Fig. 8
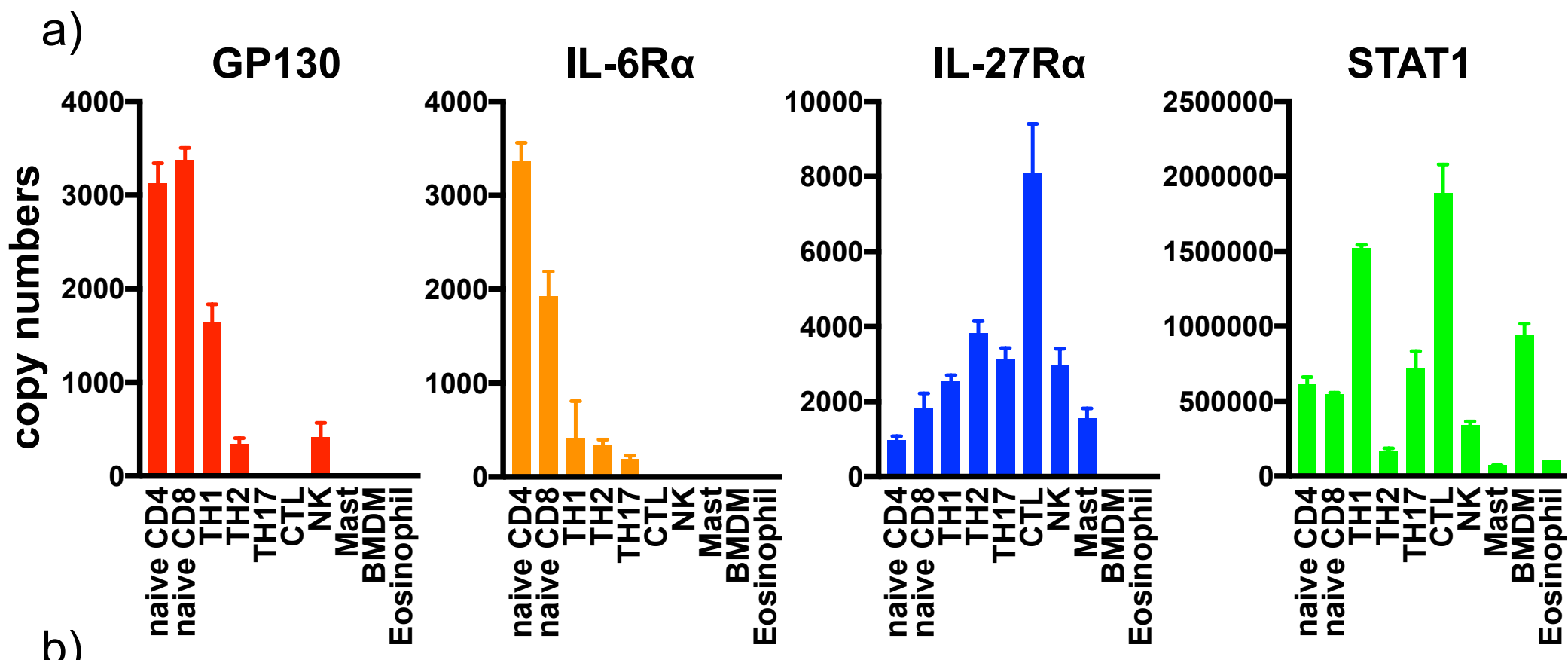

STAT3
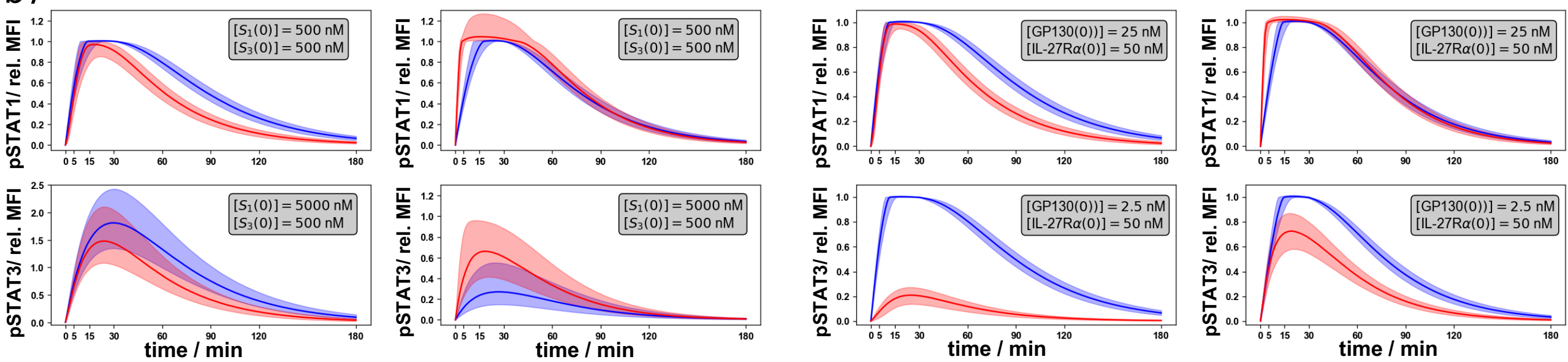

C)

- IL-27 median — HyplL-6 median $\square \mathrm{IL}-2750 \% \mathrm{Cl} \square$ HyplL-6 $50 \% \mathrm{Cl}$

— IL-27 median _ HyplL-6 median

$\square \mathrm{IL}-2750 \% \mathrm{Cl} \square$ HyplL-6 50\% Cl

$\frac{5}{\alpha}$

d)

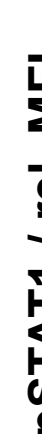

똔

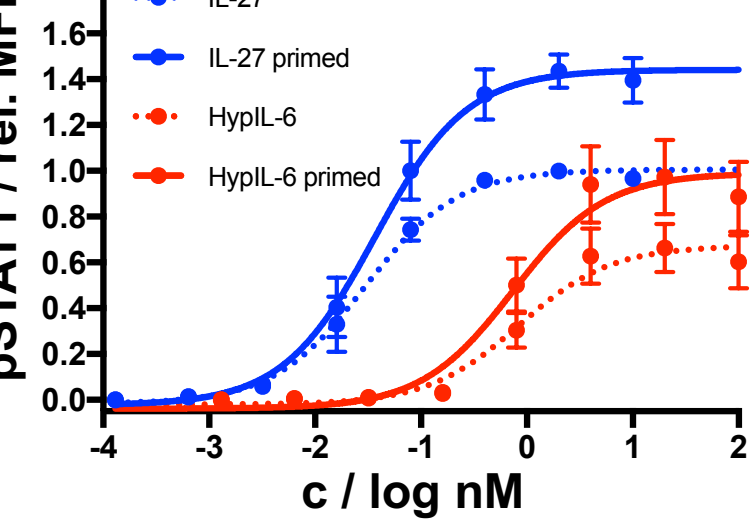

E

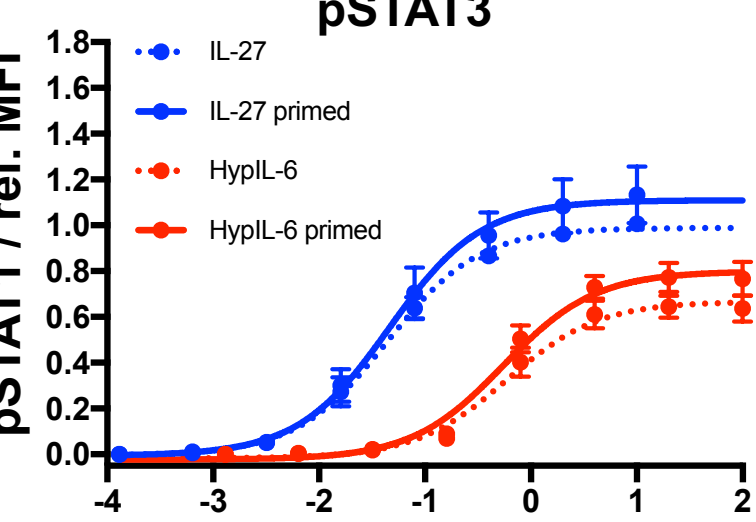

c / $\log n M$
IL-27Ra

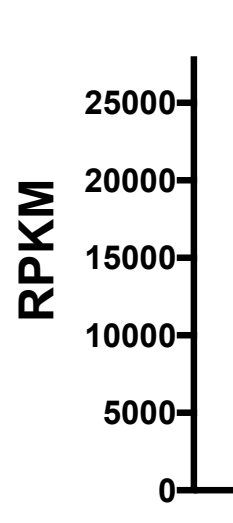

$\begin{array}{cc}\text { STAT1 } & \text { STAT3 } \\ n & * *\end{array}$

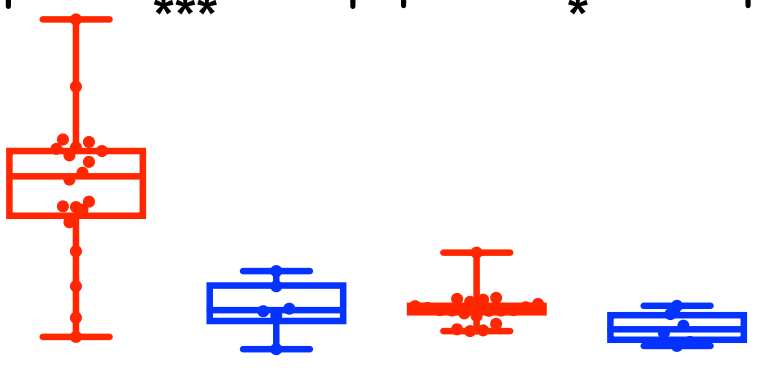

SLE dis. risk

healthy control e)

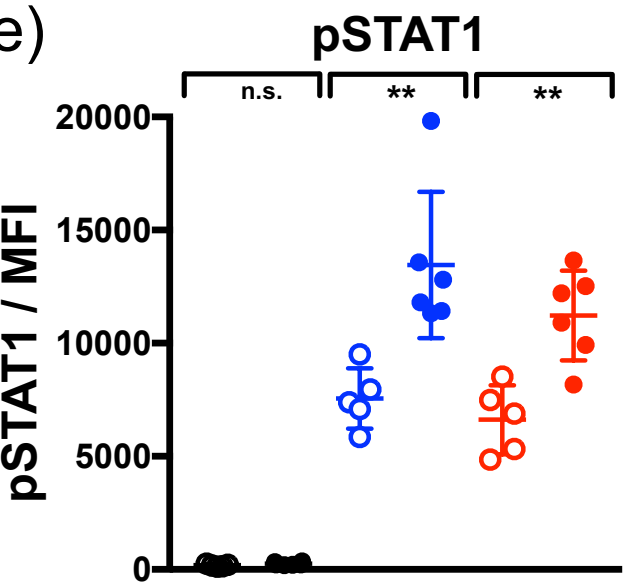

f)

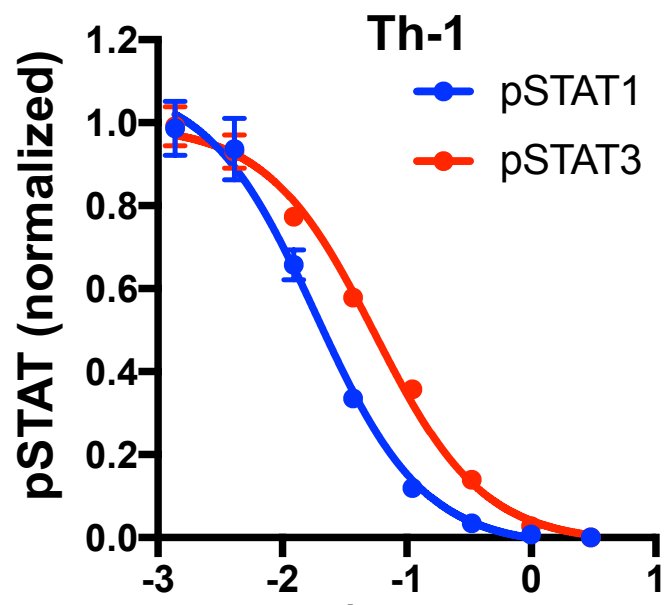

c $/ \log \mu \mathrm{M}$
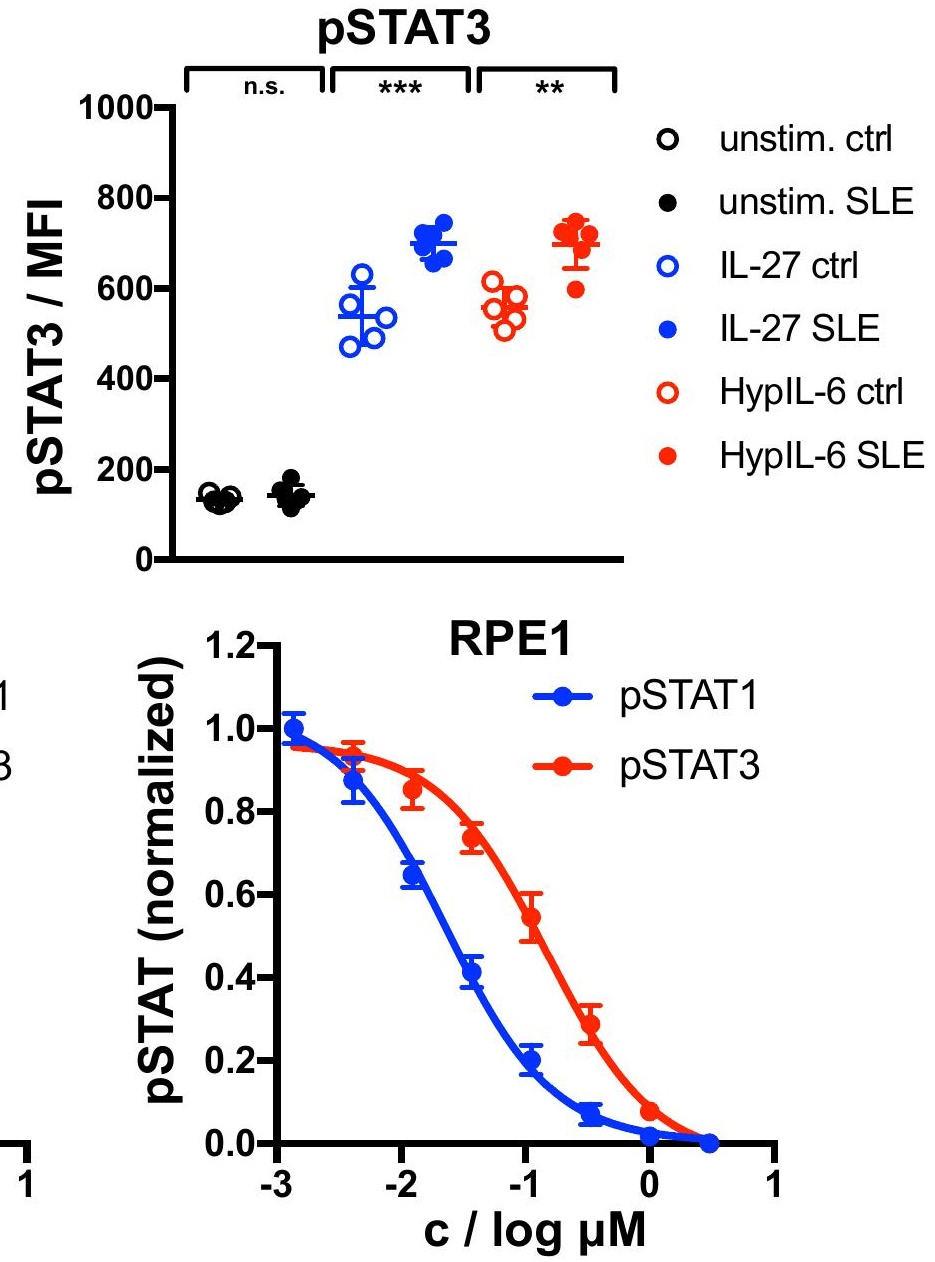
b)

I) PBMC isolation and TH1 differentiation

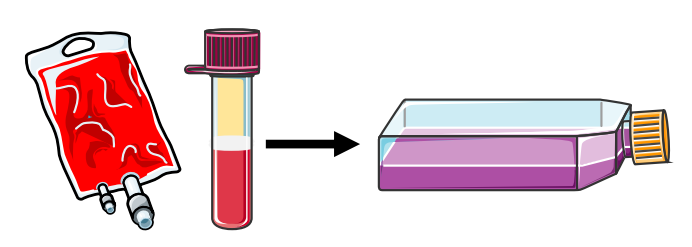

c)
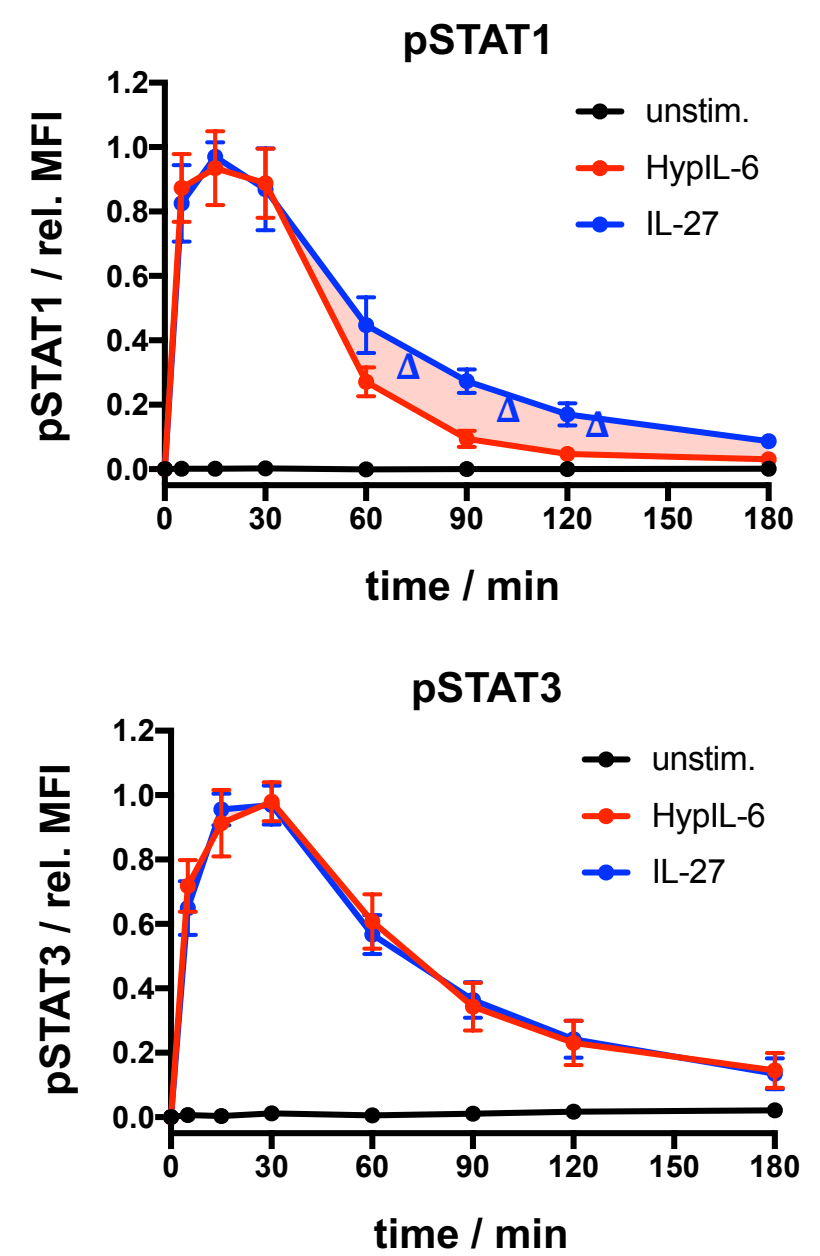

pSTAT1

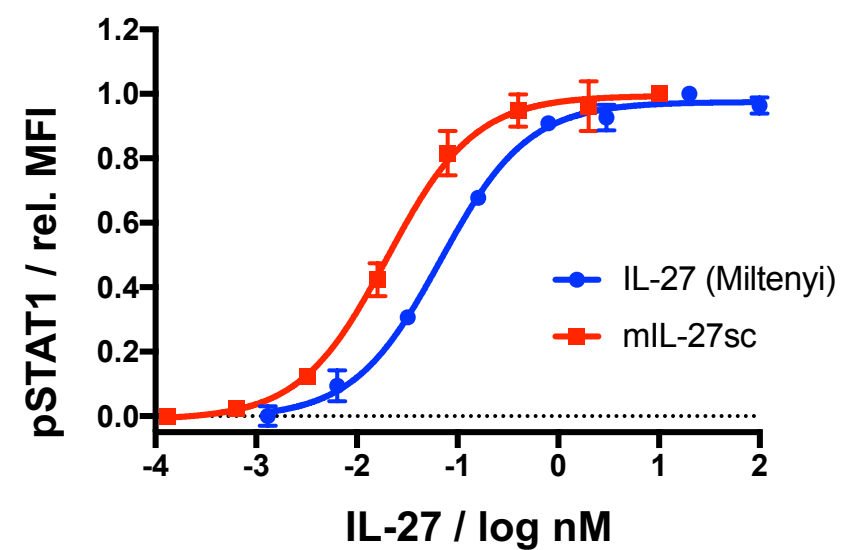

pSTAT3

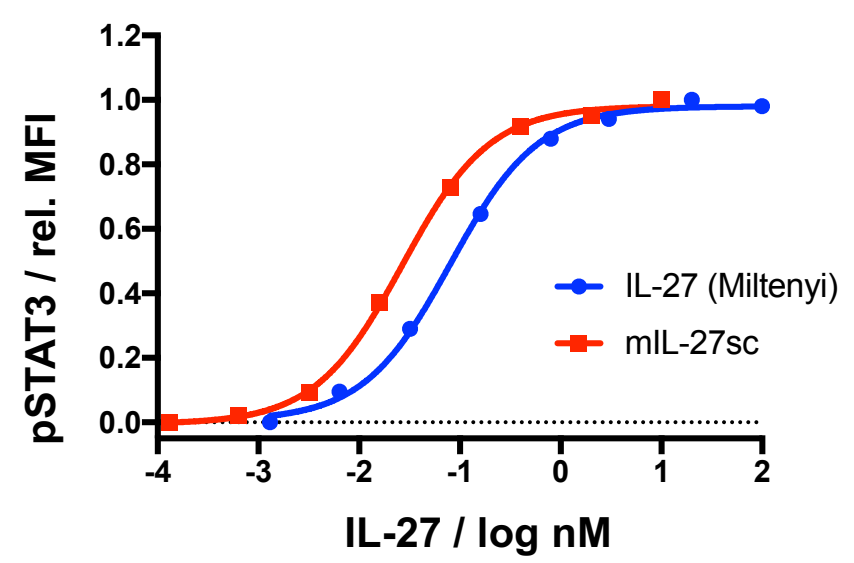

$\begin{array}{lll}\text { II) stimulation } & \text { III) merge cells }\end{array}$ \& sample barcoding \& $A B$ staining

IV) flow cytometry
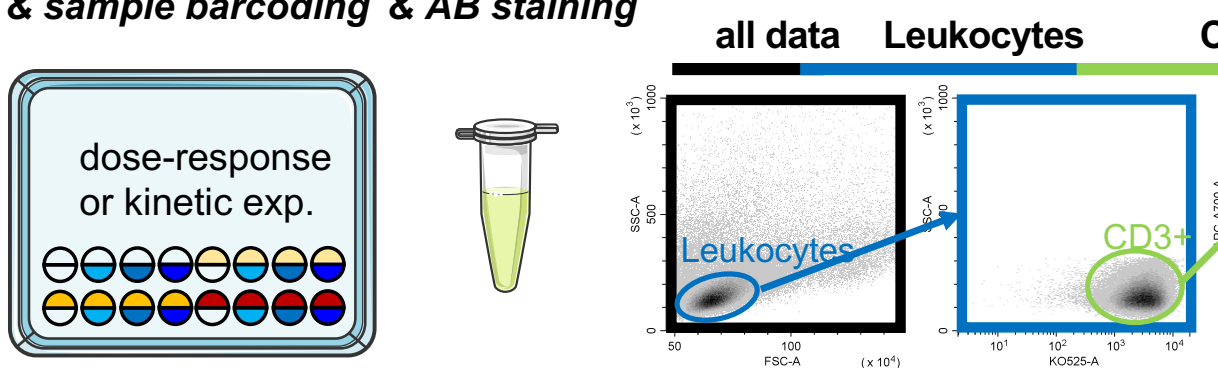

$\mathrm{CD}^{+}$

CD8-/CD4+ Barcode

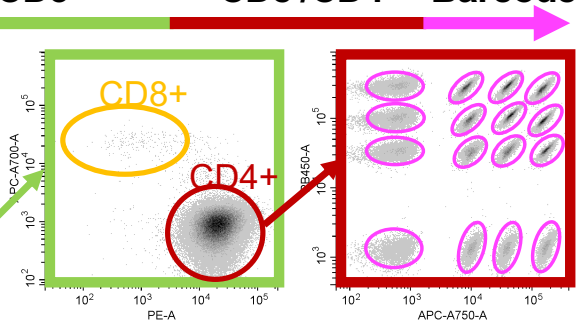

d)

CD4+

CD8+
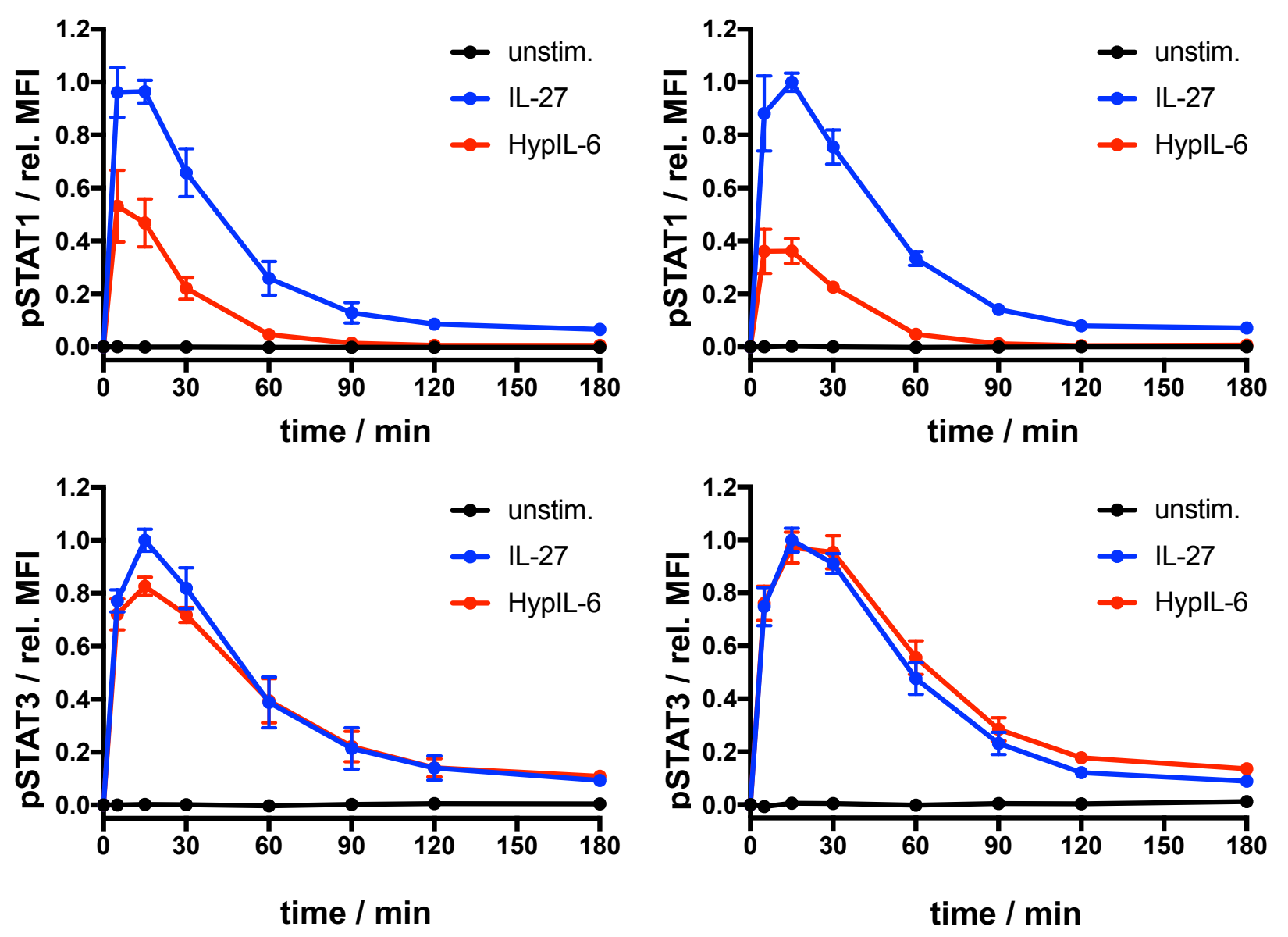

PSTAT3

e)
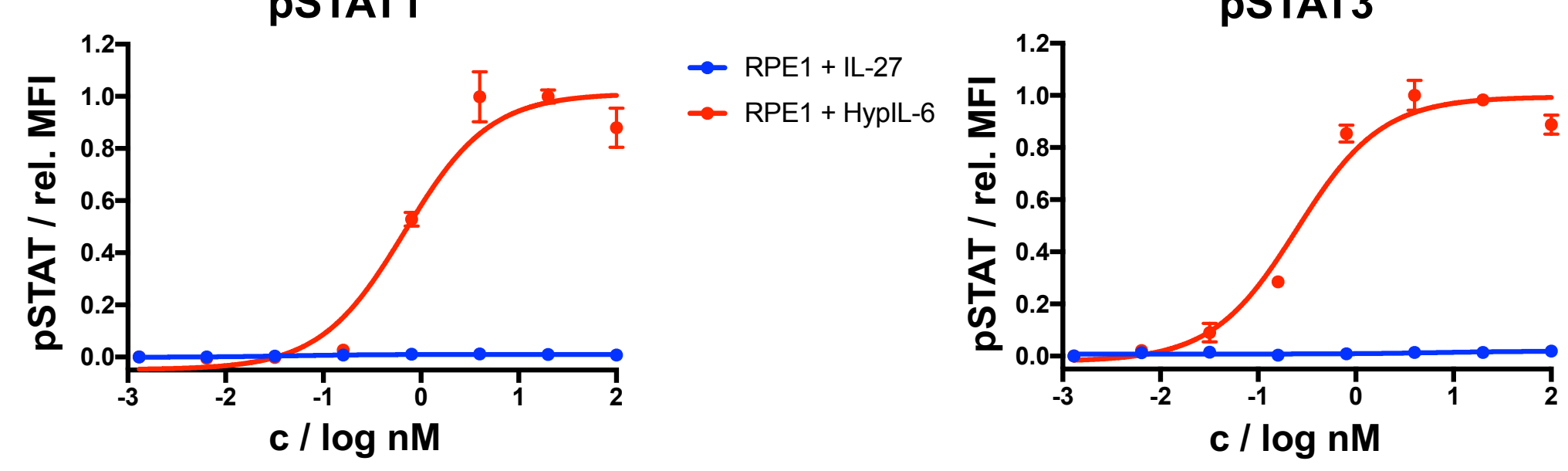

$\multimap$ RPE1 + IL-27

$\rightarrow$ RPE1 + HyplL-6 
supp. Fig. 2

a) $\quad \Delta \mathrm{GP} 130$ $\Delta \mathrm{IL}-27 \mathrm{Ra}$

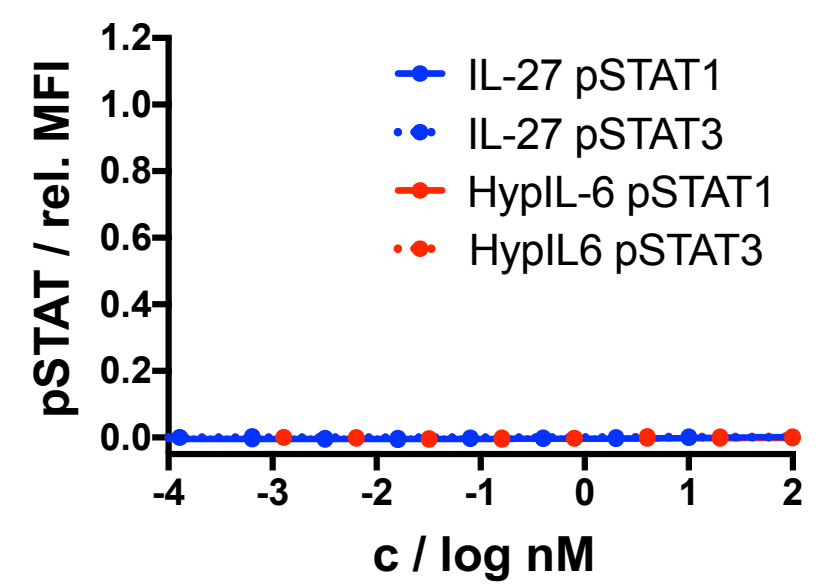

b)

Heterodimerization IL-27Ra GP130
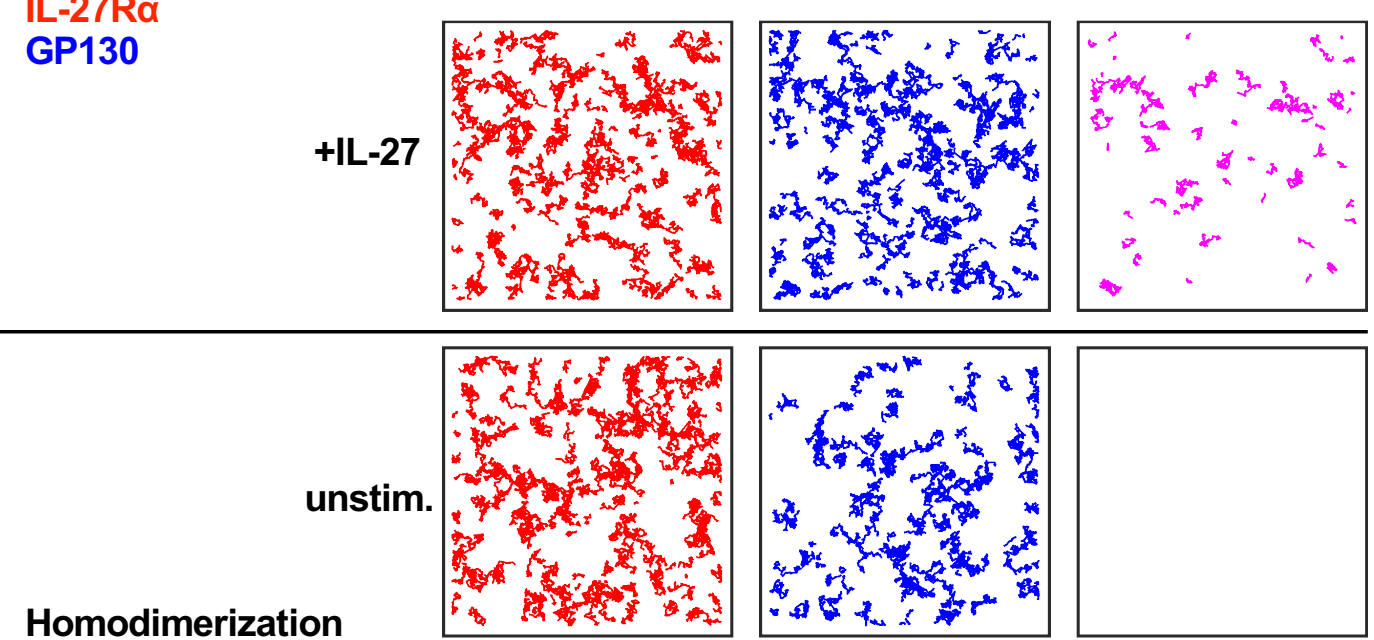

\section{GP130} GP130
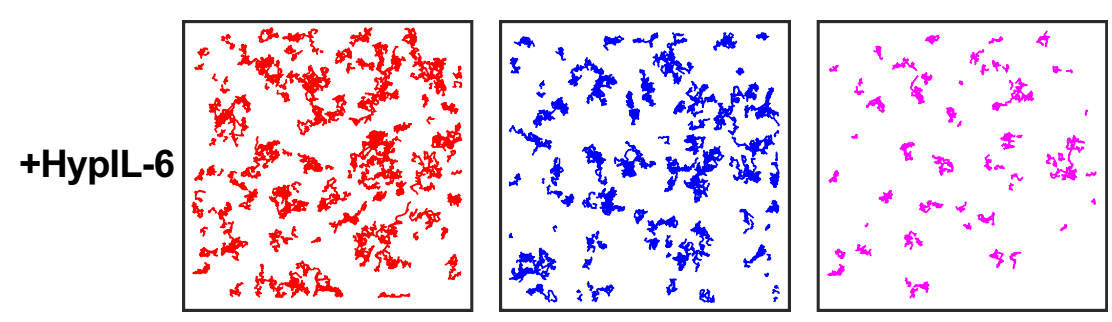

Single-color dimer

Trajectories Rho11 Trajectories DY647 Co-Trajectories

unstim.

.

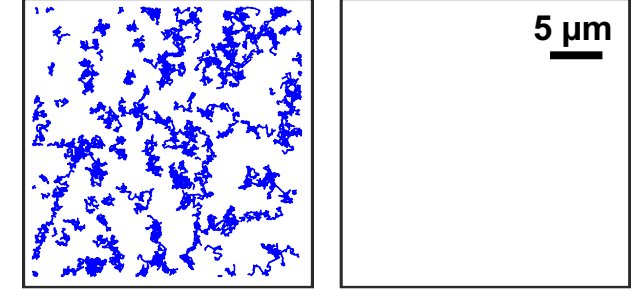

+GP130

$\Delta \mathrm{IL}-27 \mathrm{R} \alpha$

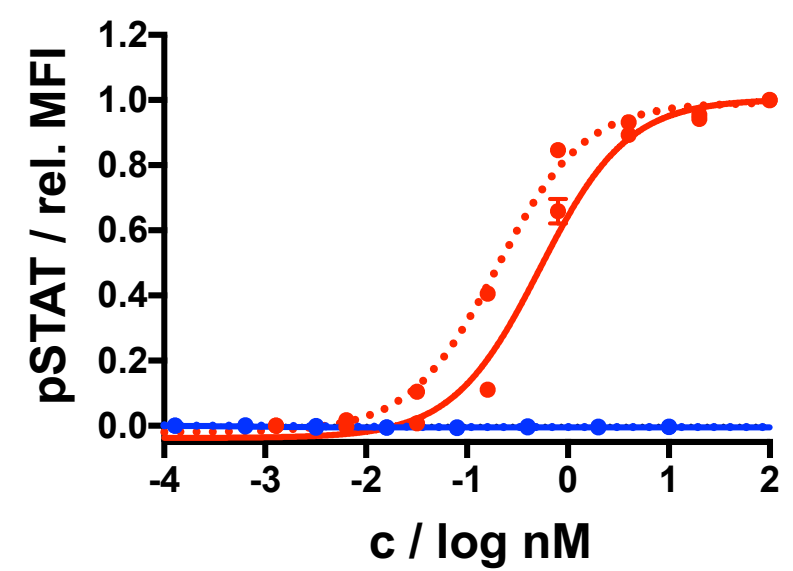

+GP130

+ IL-27R $\alpha$

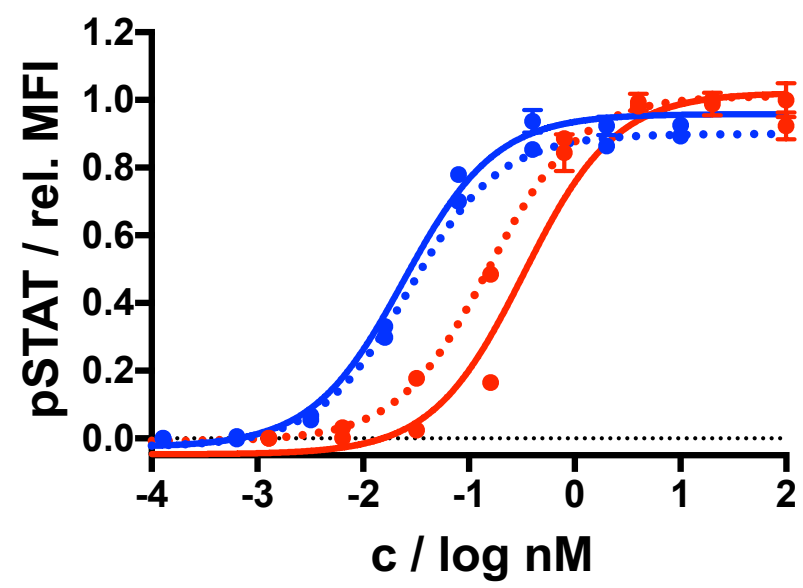

c)

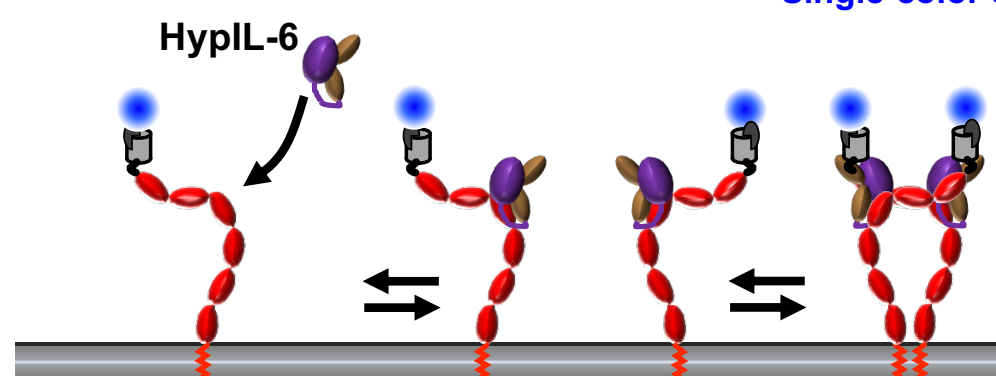

Single-color dual-step bleaching

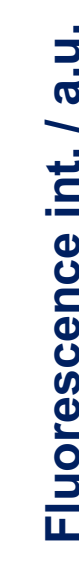
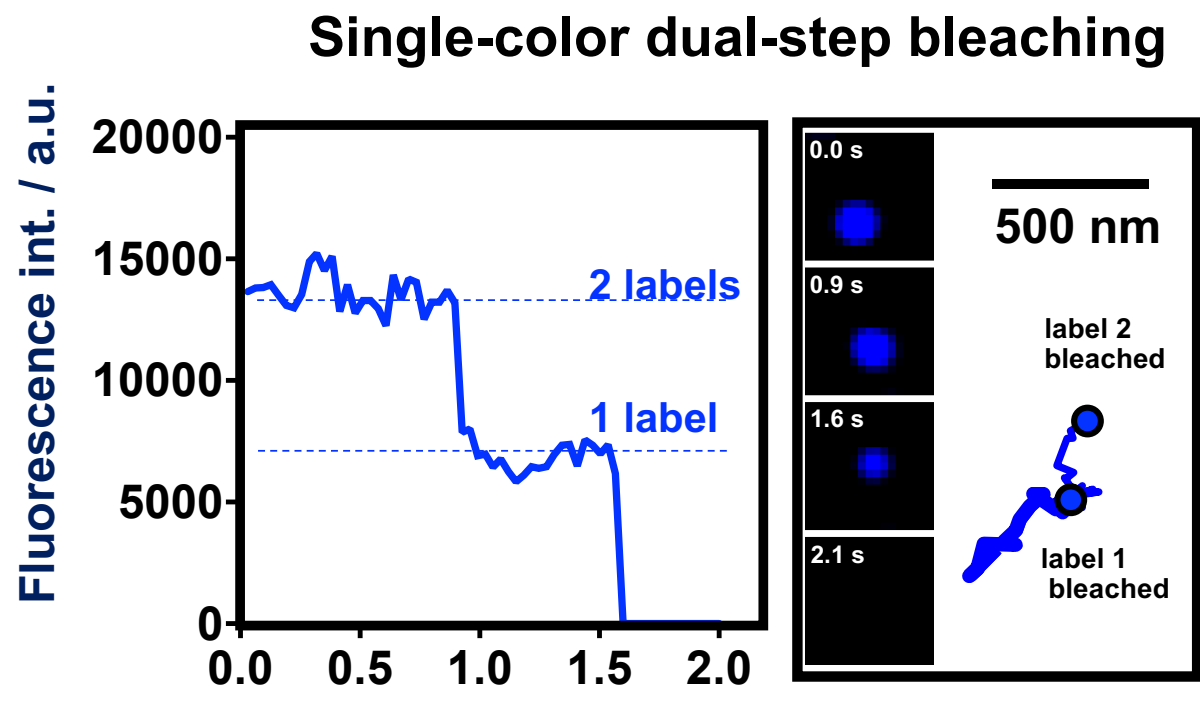

d)

is

0.10
है 0.08
0.06
0.04
0.02
0.00

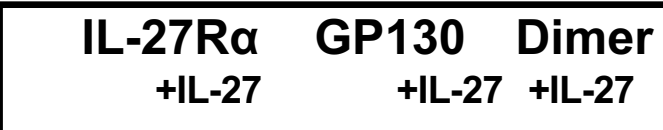

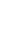
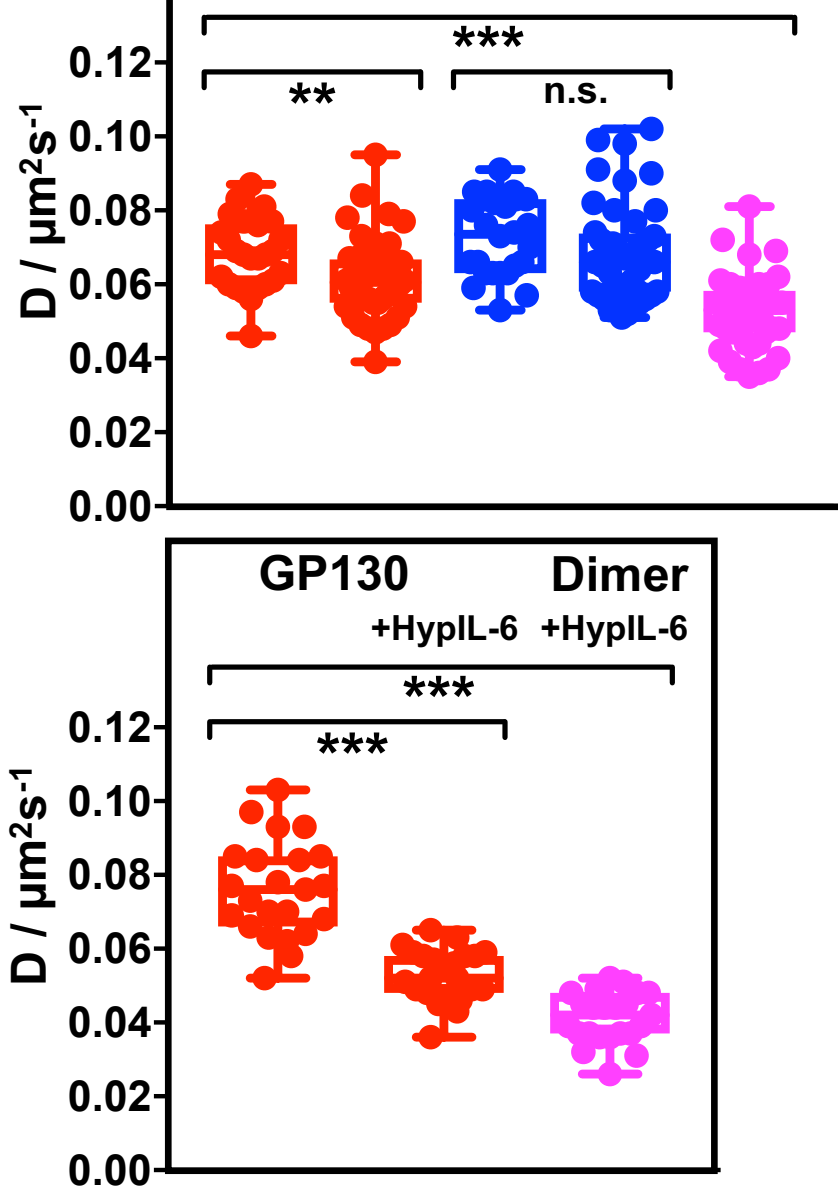

Dual-color dimer

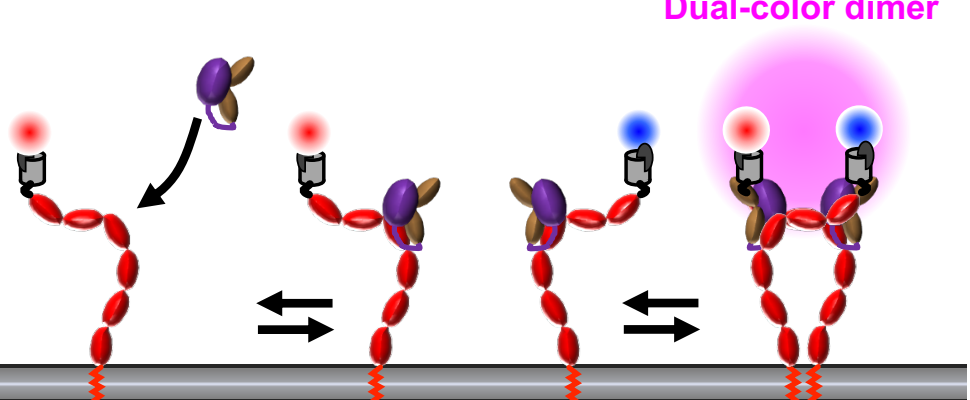

Dual-color single-step bleaching time / s
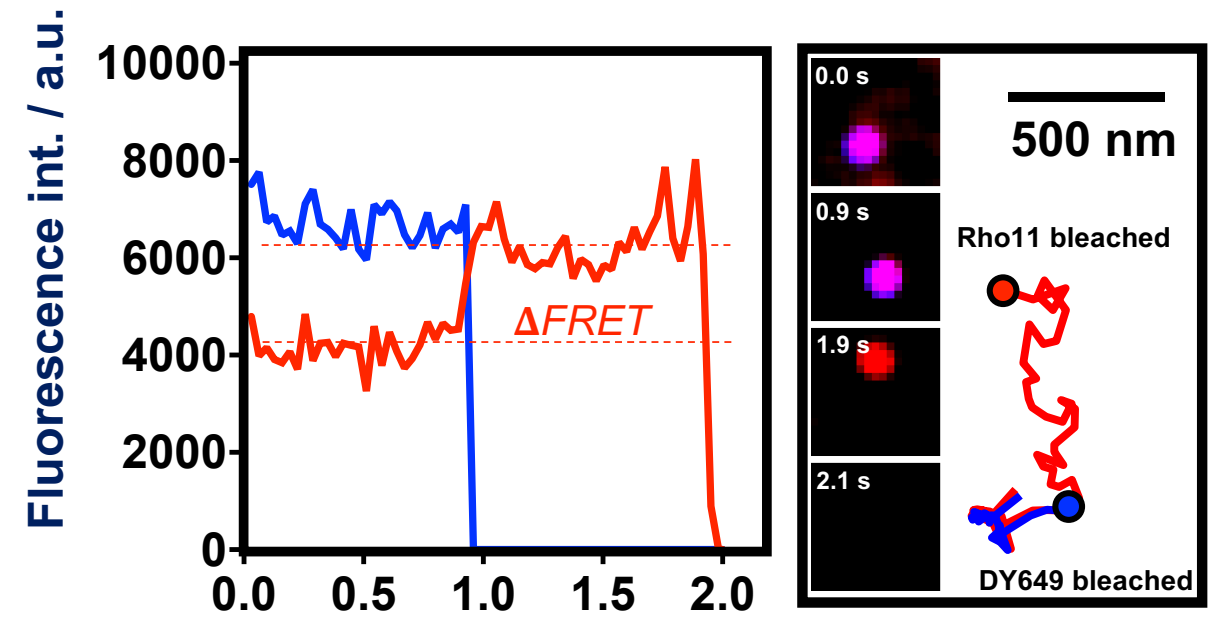
time / s 
a)

$$
\begin{aligned}
& \text { 吕 }+ \text { • } \frac{r_{1,6}^{+}}{r_{1,6}^{-}} \\
& \text {它 }+\stackrel{\Downarrow}{\frac{r_{2,6}^{+}}{r_{2,6}^{-}}}
\end{aligned}
$$

c)

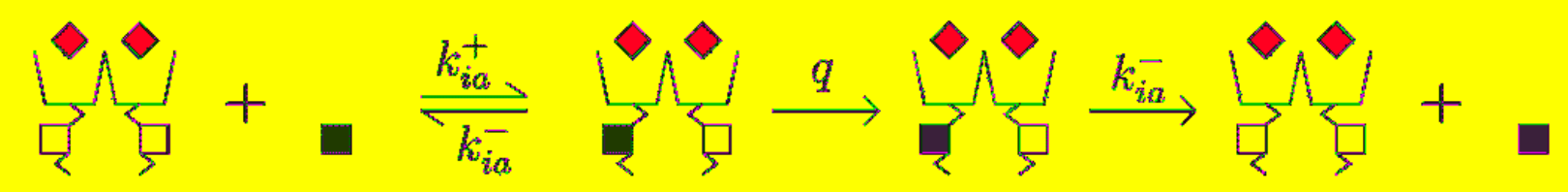

官 + - $\stackrel{k_{i a}^{+}}{\stackrel{k_{i a}^{-}}{*}} \stackrel{q}{\longrightarrow}$

客 + - $\stackrel{k_{i a}^{+}}{k_{i a}^{-}}$

d)

尚) 岁 + - $\stackrel{k_{i a}^{+}}{k_{i a}^{-}}$

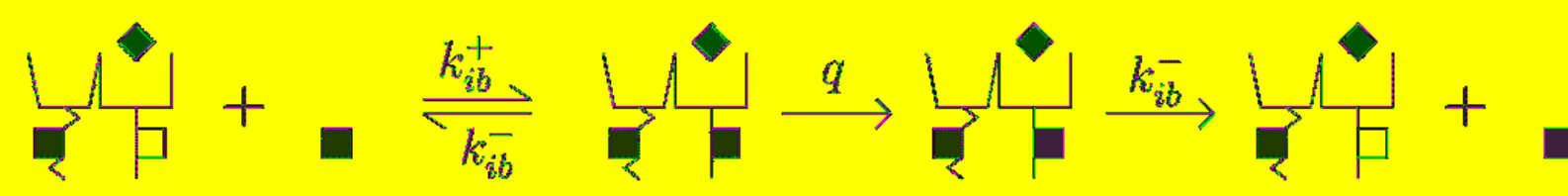

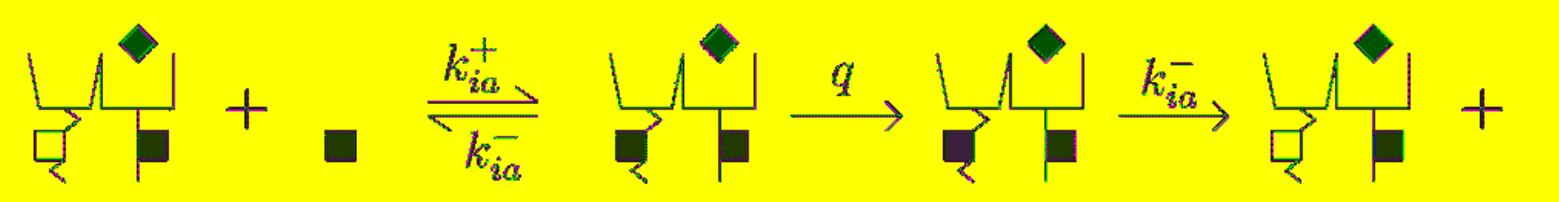

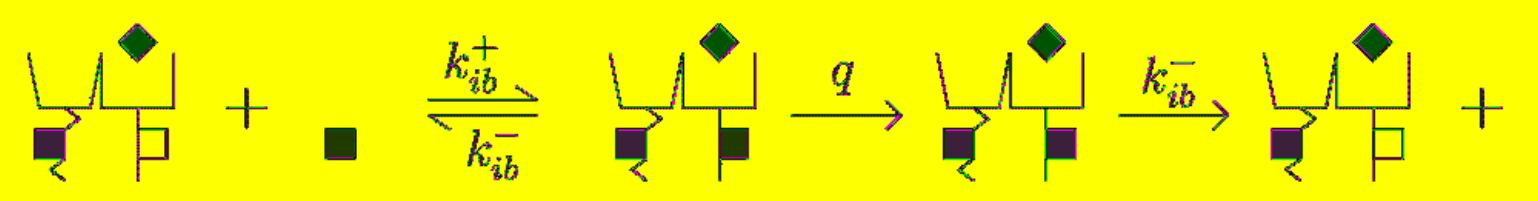

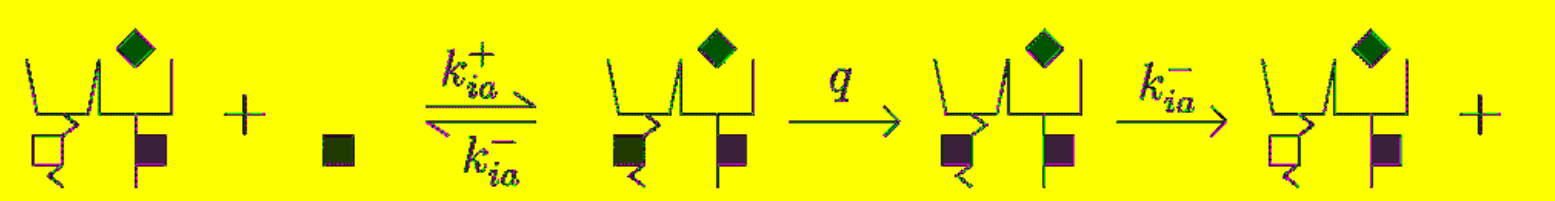

e)

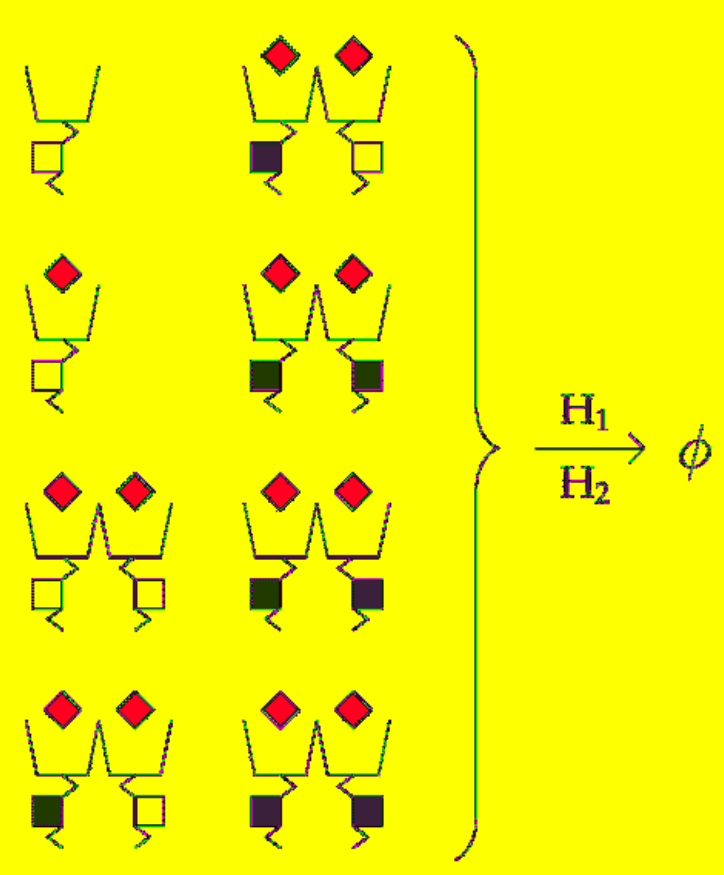

f)

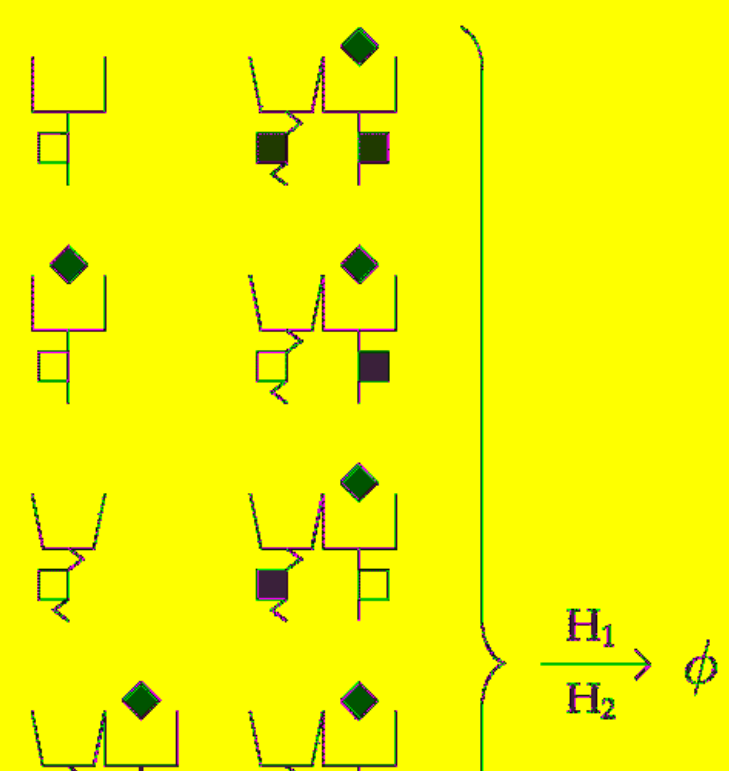

g) 눙분

h)

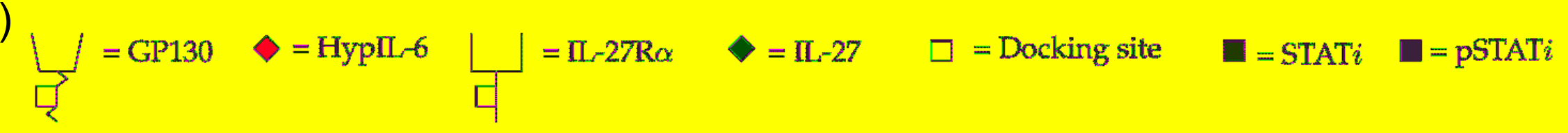


a)

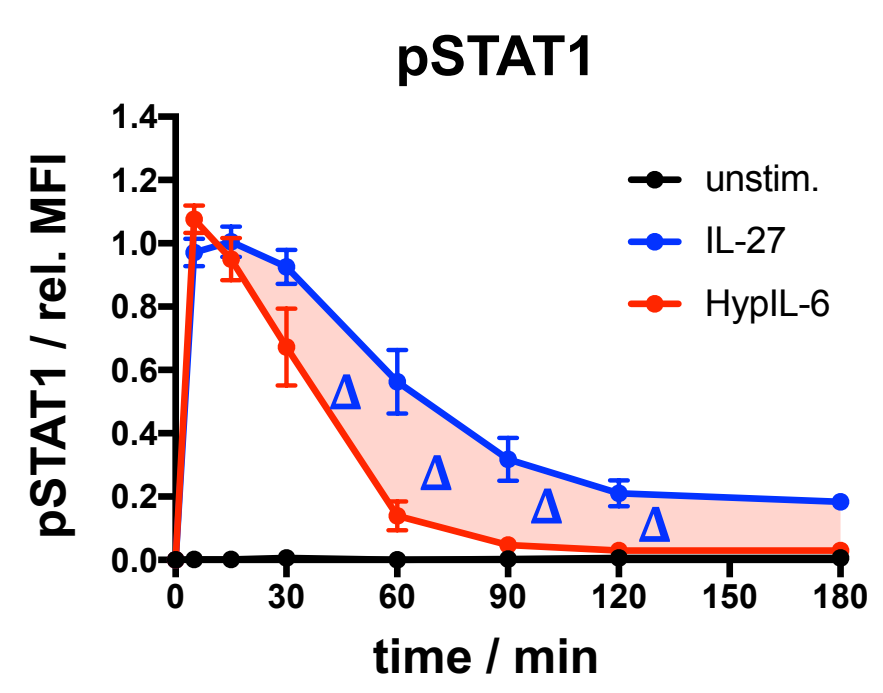

b)

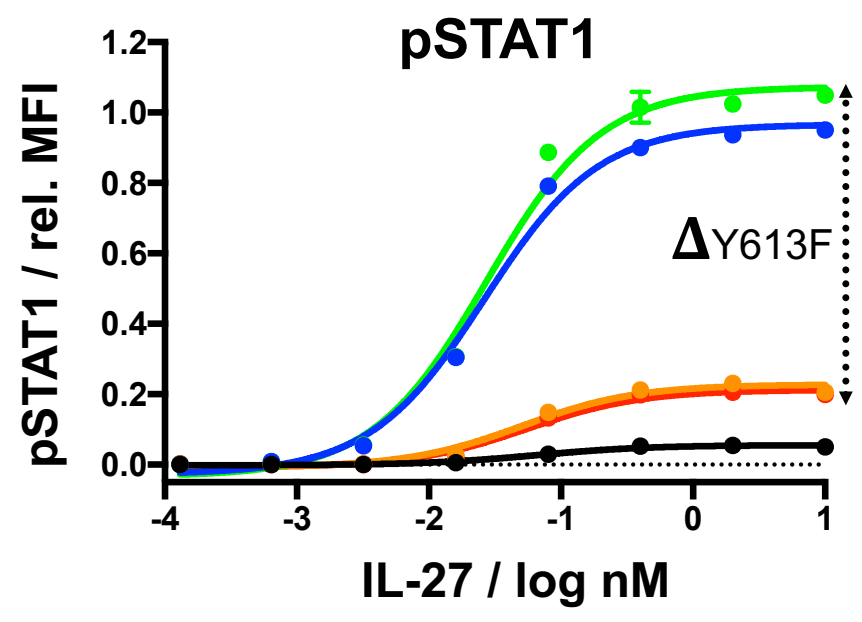

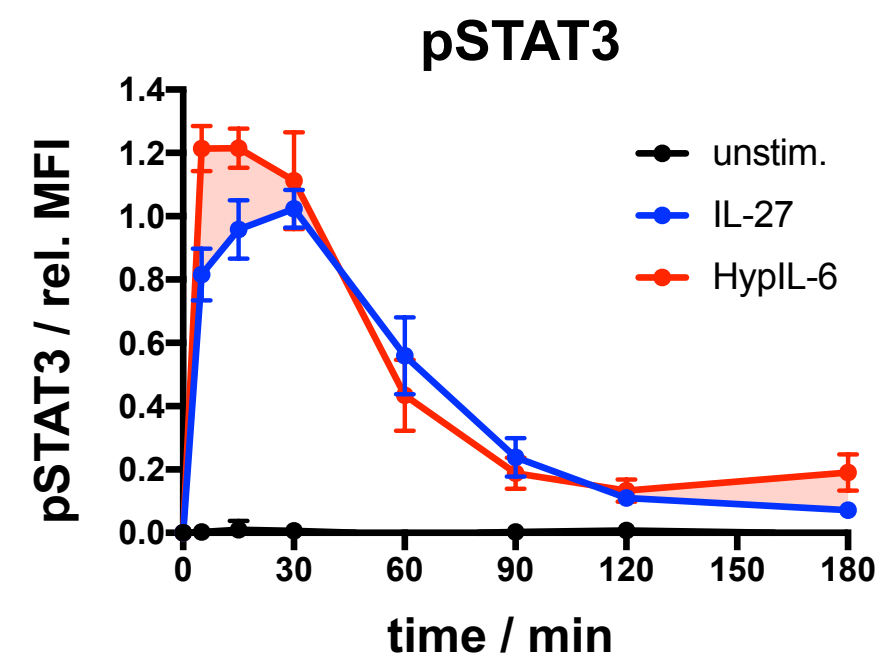

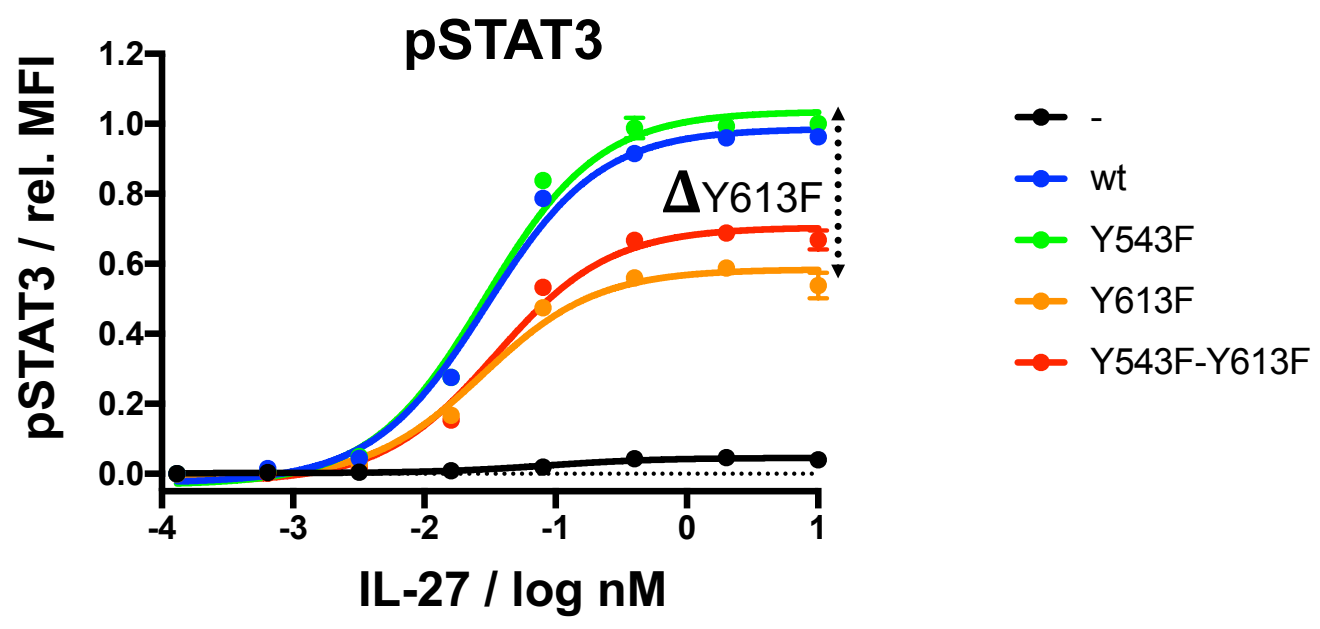




\section{supp. Fig. 5}

a)
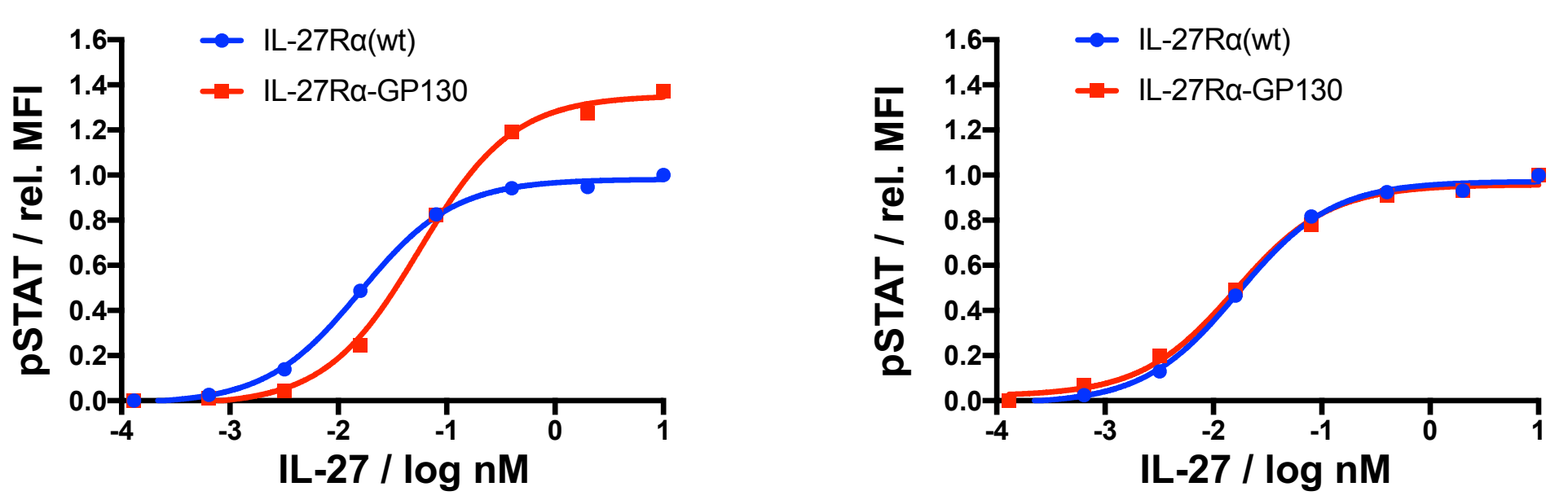

b)

$\underline{R P E 1 ~ I L-27 R \alpha ~ c e l l s ~}$
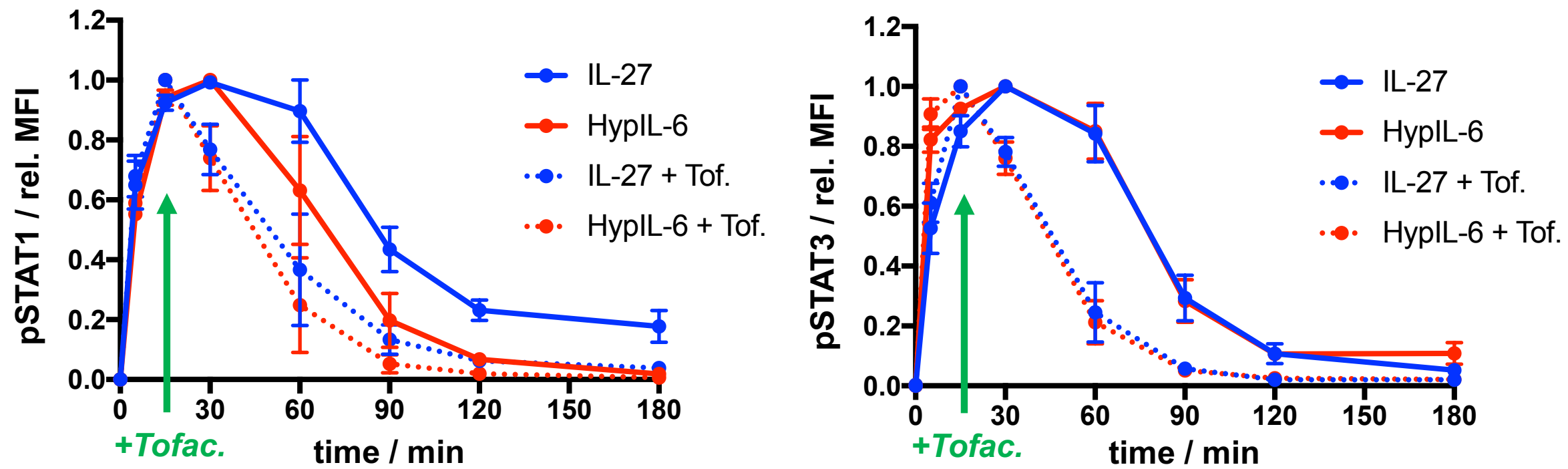

c)

$\underline{\text { TH1 cells }}$
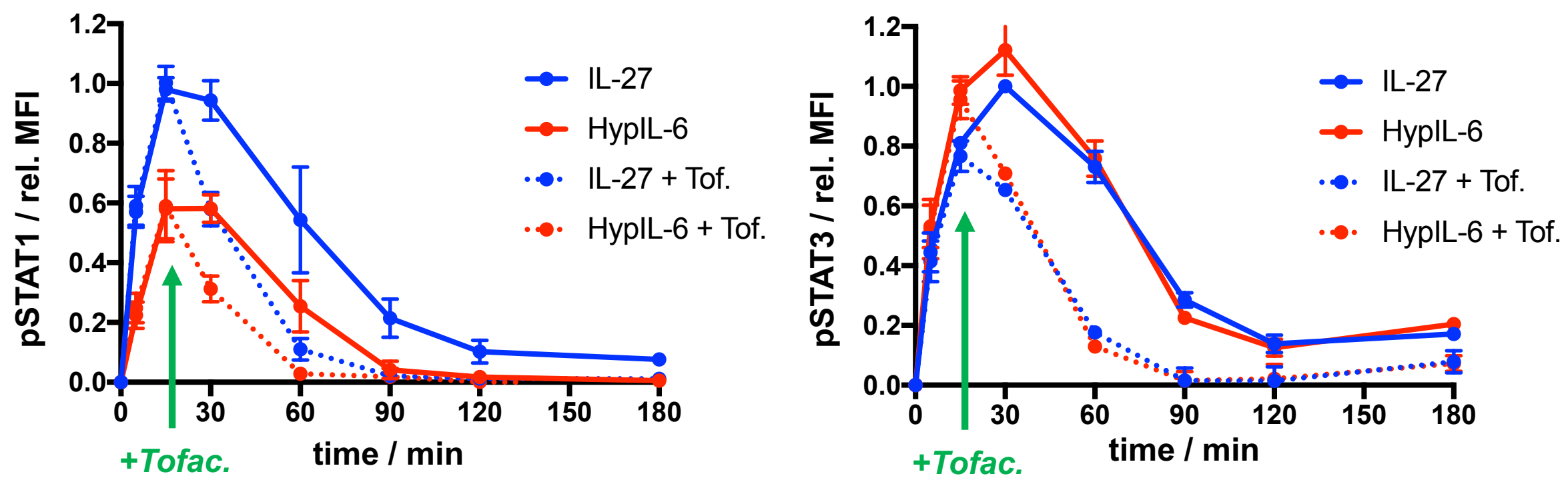

d)

$\underline{\text { TH1 cells (ratio +/- Tofacitinib) }}$
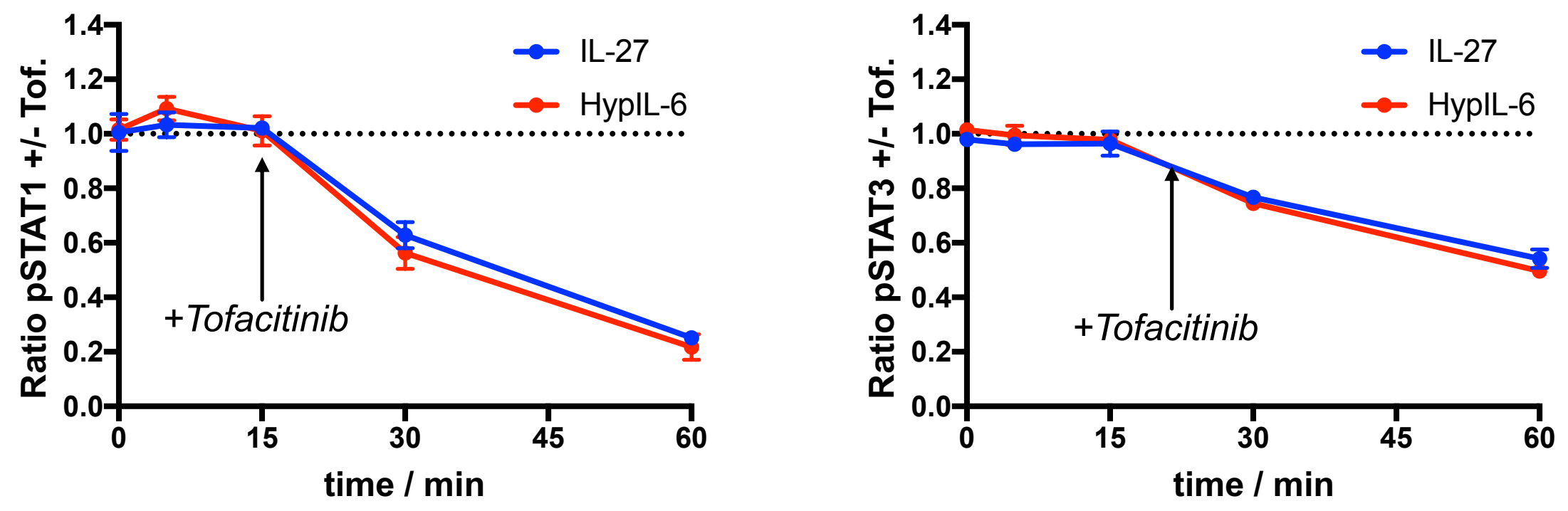
a)

RPE1 IL-27Ra cells
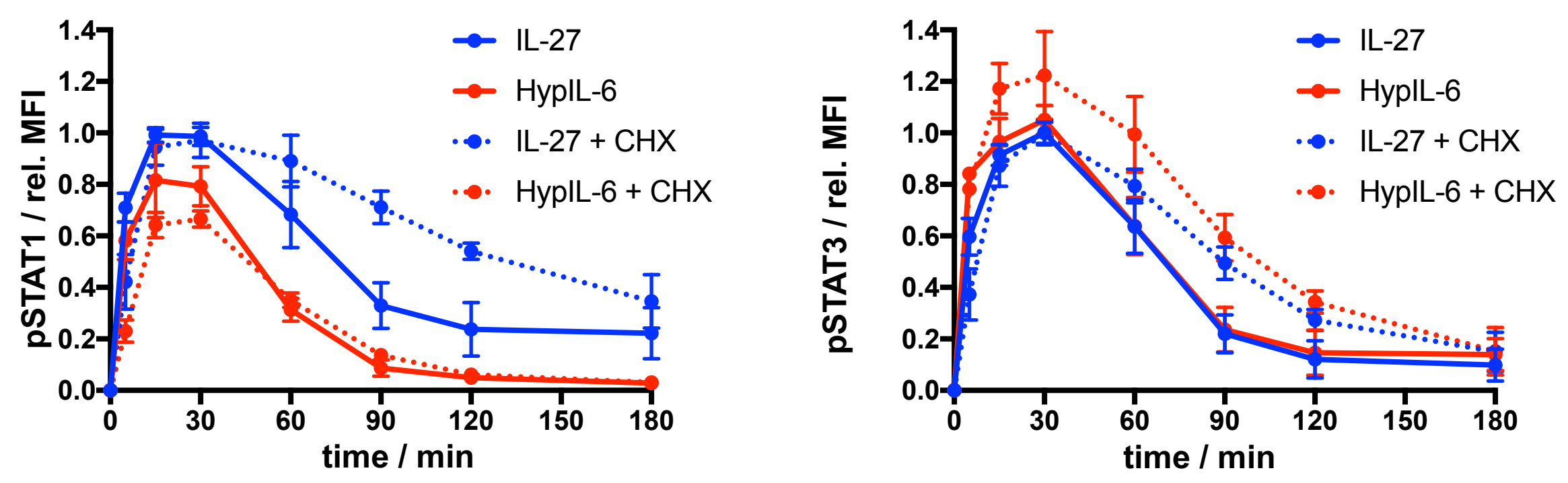

b)

$\underline{\text { TH1 cells }}$
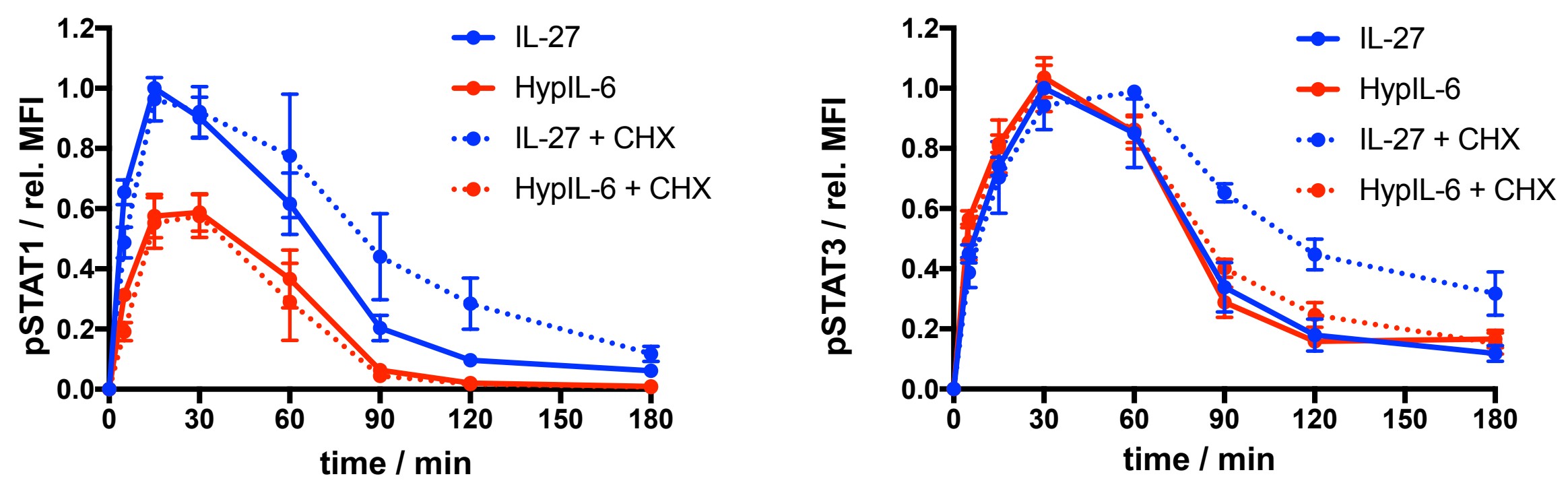


\section{supp. Fig. 7}

a) Isolate PBMCs

From buffy coat

Differentiate to Th-1 Stimulation:

Mix 1:1 cell numbers Lyse

Phosphopeptide

Enrichment

LC-MS/MS \& CD4+ isolation

In SILAC media $15 \mathrm{~min}$

$\left(\mathrm{TiO}_{2}\right)$
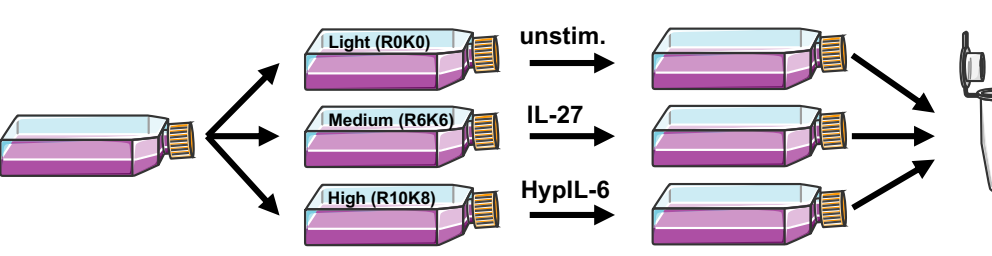

Alkylate

b)

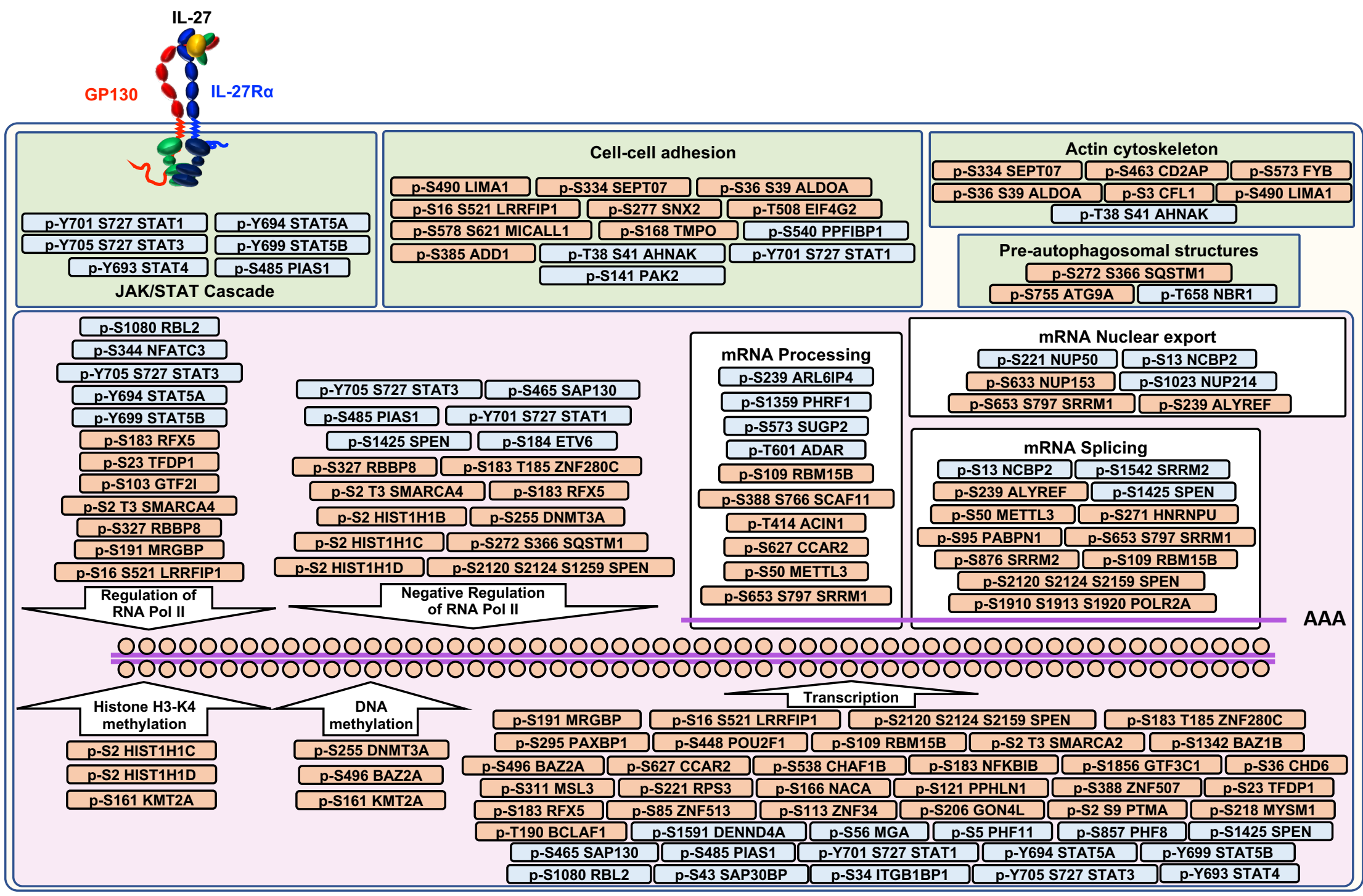

c)

Cell-cell adhesion

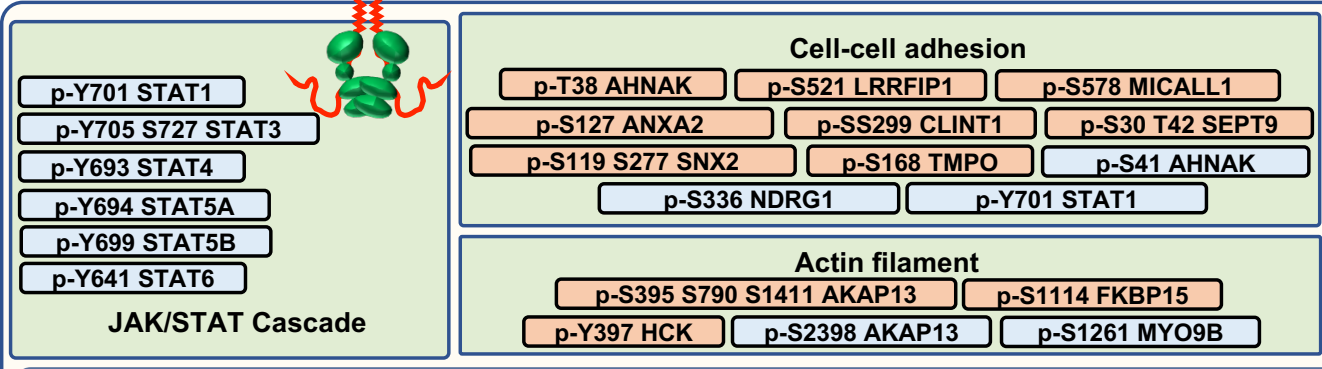
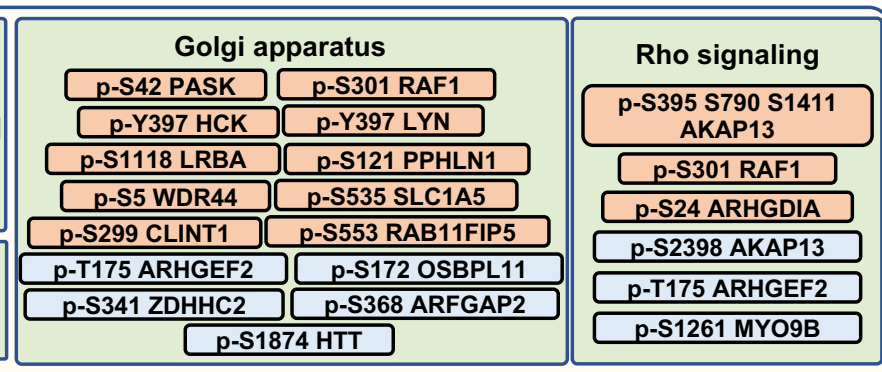

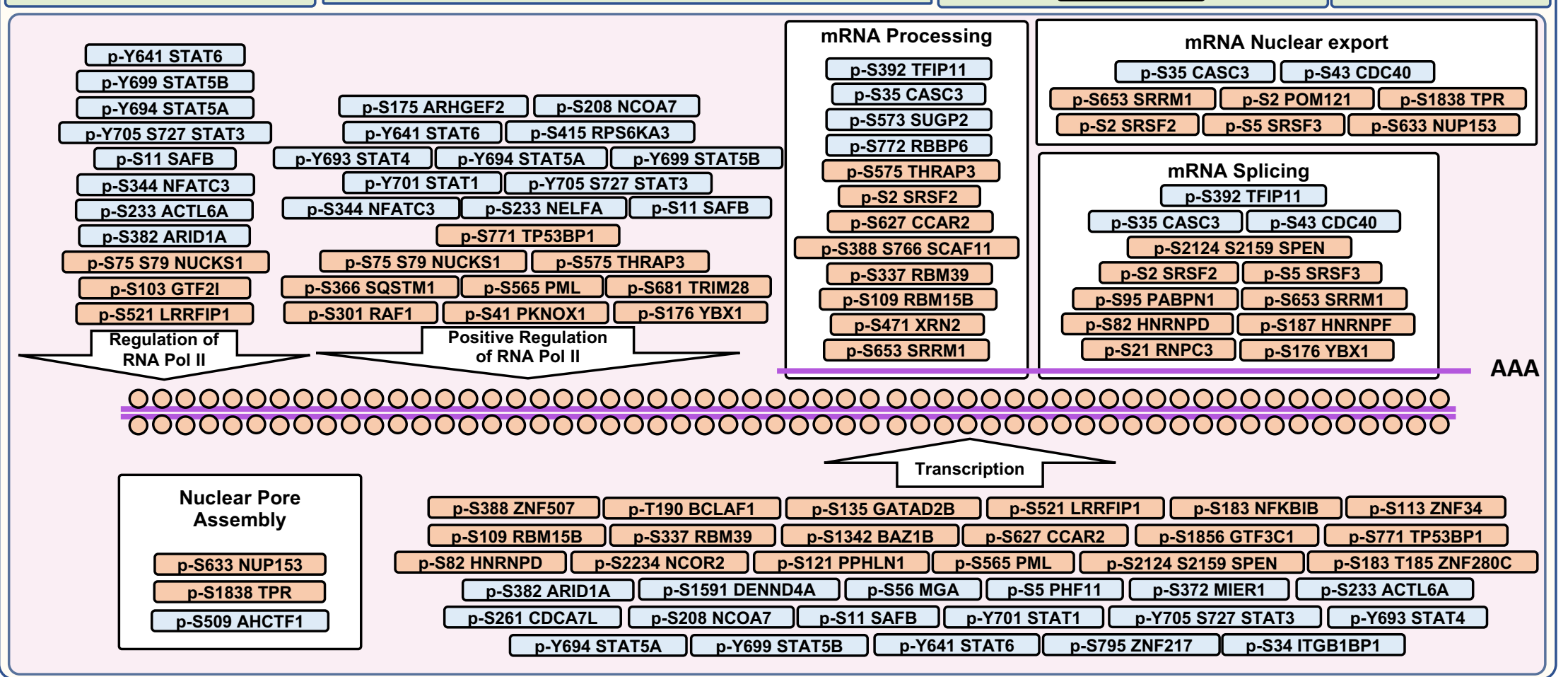




\section{supp. Fig. 8}

a)

Downregulated phospho-sites

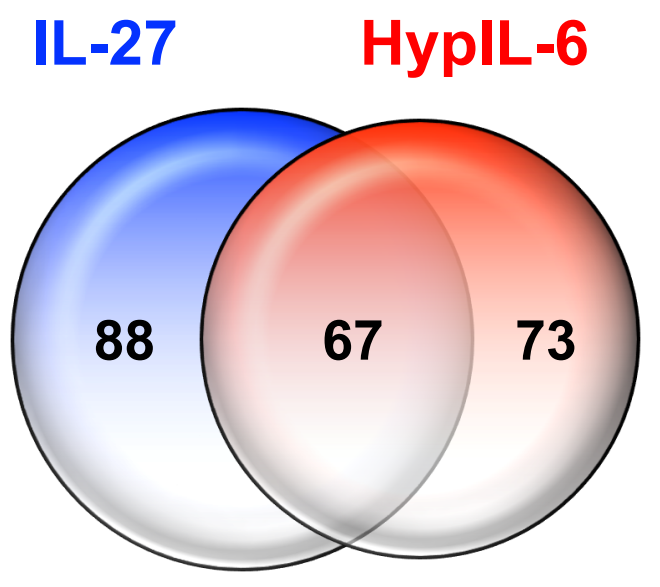

b)

\section{IL-27}

\begin{tabular}{|l|r|l|r|}
\hline peptide & $\begin{array}{r}\text { Fold change } \\
/ \log _{2}\end{array}$ & peptide & Fold change \\
$/ \log _{2}$ \\
\hline CHD12 S144 & -6.33 & LGALSL S4 & 9.05 \\
\hline MAP1B S2271 & -3.66 & RNASE9 S53 T54 & 5.73 \\
\hline ZNF280C S183 T185 & -3.16 & AHNAK S41 T38 & 4.00 \\
\hline ADGRF2 T601 Y588 & -3.11 & BAD S25 & 3.99 \\
\hline ZC2HC1A S223 & -2.39 & CLK3 S157 & 3.74 \\
\hline BOLA1 S81 & -2.30 & STAT4 Y693 & 3.67 \\
\hline GTF2I S103 & -2.25 & DCP1B S283 & 3.47 \\
\hline TACC1 S689 Y695 & -2.17 & STAT3 Y705 & 2.81 \\
\hline SCAF11 S776 & -2.08 & STAT1 Y701 & 2.63 \\
\hline ABCC1 S915 & -1.97 & STAT5A/B Y694/Y699 & 2.18 \\
\hline WRNIP1 S151 & -1.95 & PTPN11 Y546 & 1.93 \\
\hline SEC23IP S737 & -1.92 & BAD S134 & 1.84 \\
\hline RBM15B S109 & -1.81 & ARL6IP4 S239 & 1.78 \\
\hline MECP2 S25 & -1.65 & UBR5 S1549 & 1.77 \\
\hline PSMD11 S14 & -1.63 & PIEZO1 S1646 & 1.70 \\
\hline OSPBL8 S68 & -1.40 & PPM1G T122 & 1.69 \\
\hline
\end{tabular}

c)

\section{Cellular location}

IL-27 HypIL-6

Golgi apparatus Golgi apparatus Actin filament Actin cytoskeleton mRNA transport Chromatin regulator mRNA processing

Transcription Cytoplasm Methylation Nucleus

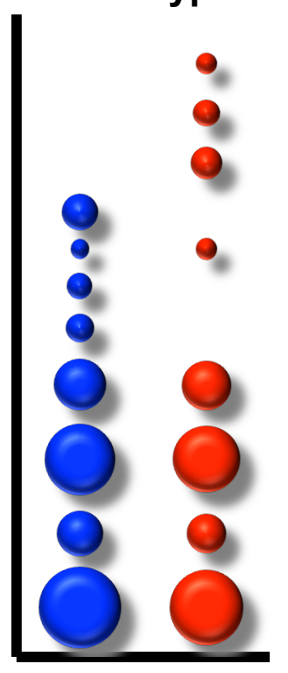

\section{Upregulated phospho-sites}
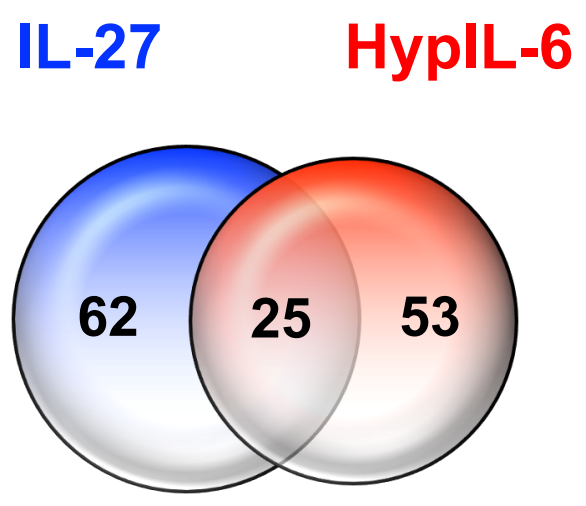

HyplL-6

\begin{tabular}{|l|r|l|r|}
\hline peptide & $\begin{array}{r}\text { Fold change } \\
/ \log _{2}\end{array}$ & peptide & $\begin{array}{r}\text { Fold change } \\
/ \log _{2}\end{array}$ \\
\hline TACC1 S689 Y695 & -4.88 & LGALSL S4 & 6.49 \\
\hline CDH12 S144 & -4.16 & STAT4 Y693 & 5.74 \\
\hline MAP1B S2271 & -4.01 & MYO9B S1261 & 4.34 \\
\hline ZNF280C S183 T185 & -3.42 & ANKRD36C T828 & 4.30 \\
\hline ADGFR2 T601 Y588 & -3.37 & CDCA7L S261 & 3.54 \\
\hline ZC2HC1A S223 & -2.46 & STAT3 Y705 & 3.40 \\
\hline BOLA1 S81 & -2.44 & NELFA S233 & 2.92 \\
\hline WRNIP1 S151 & -2.40 & PPM1G T122 & 2.90 \\
\hline FAM47E T158 Y161 & -2.17 & BAD S25 & 2.84 \\
\hline SCAF11 S776 & -2.15 & NDRG1 S336 & 2.79 \\
\hline ABCC1 S915 & -2.07 & STAT1 Y701 & 2.69 \\
\hline NUDT19 S4 & -1.97 & SUGP2 S573 & 2.18 \\
\hline GTF2I S103 & -1.85 & PRR12 S44 & 1.98 \\
\hline ZC3H3 S408 & -1.69 & STAT3 S727 & 1.97 \\
\hline SEC23IP S737 & -1.64 & PTPN11 Y546 & 1.73 \\
\hline PSMD11 S14 & -1.60 & RCHY1 S257 & 1.72 \\
\hline
\end{tabular}

UP keywords

d)
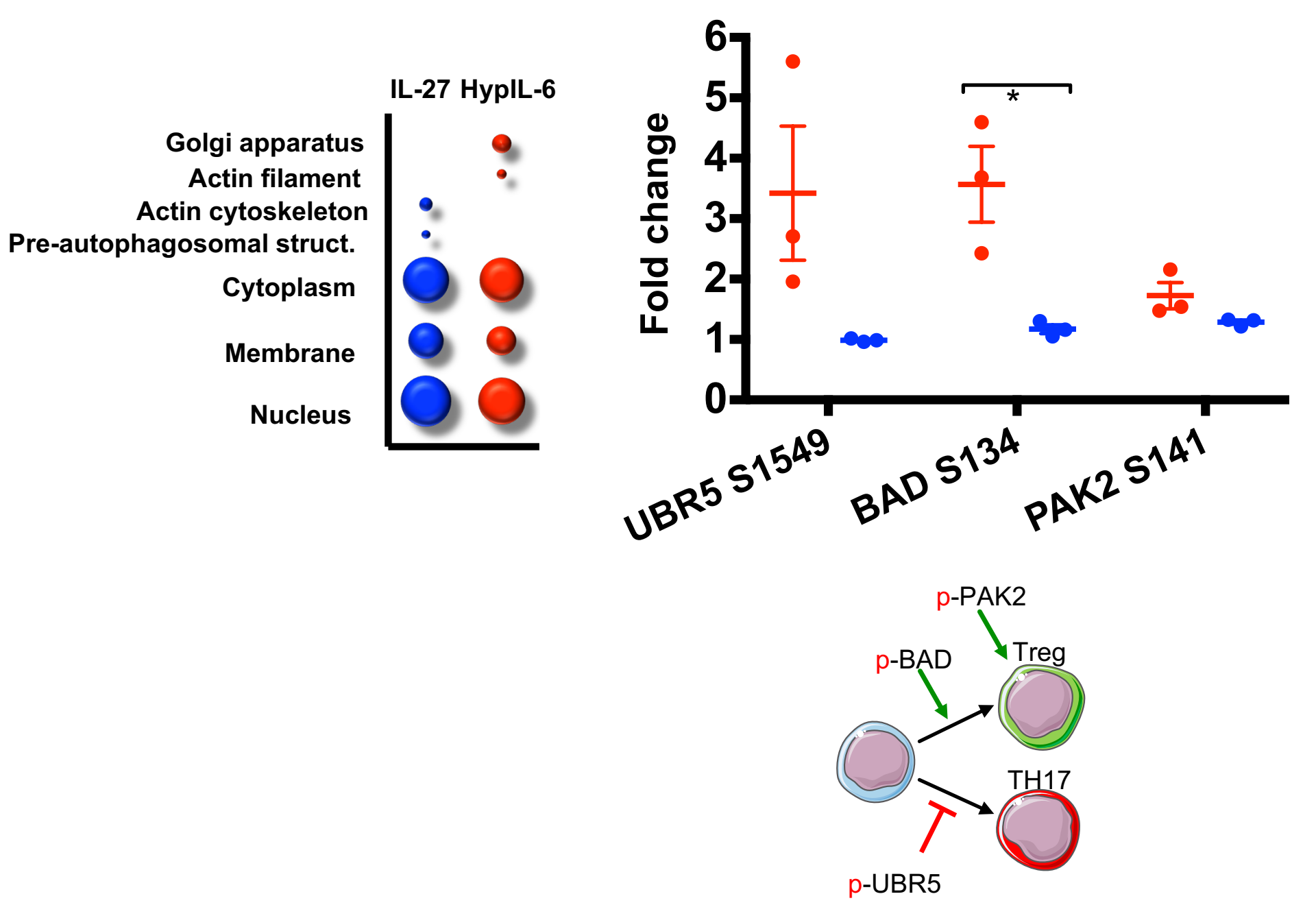
supp. Fig. 9

a)
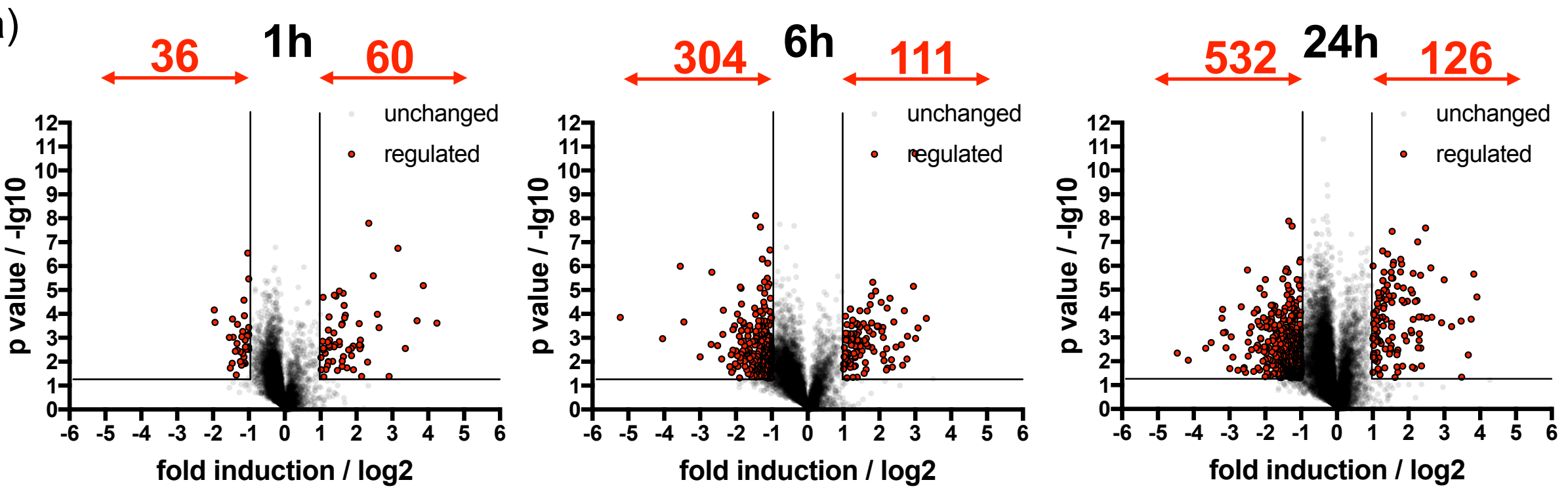

b)

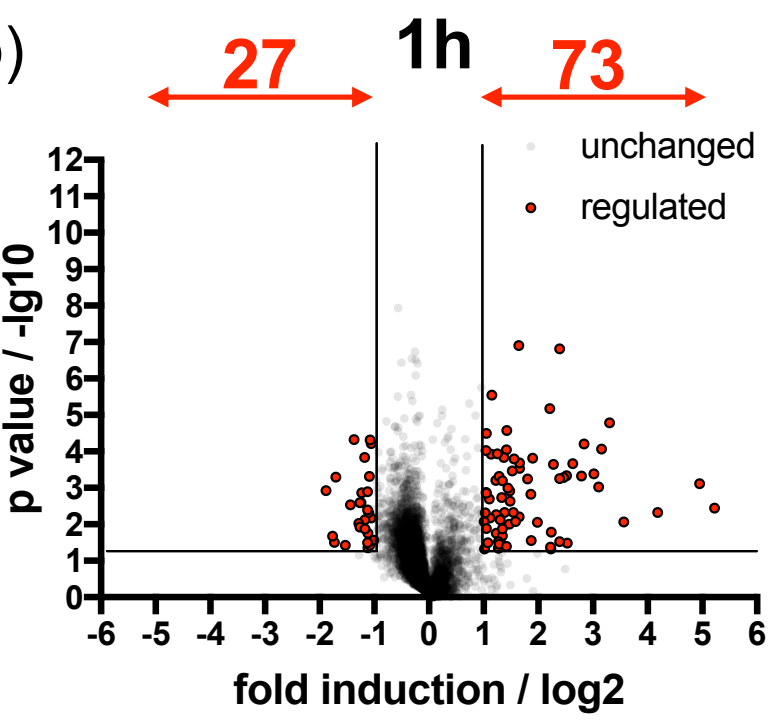

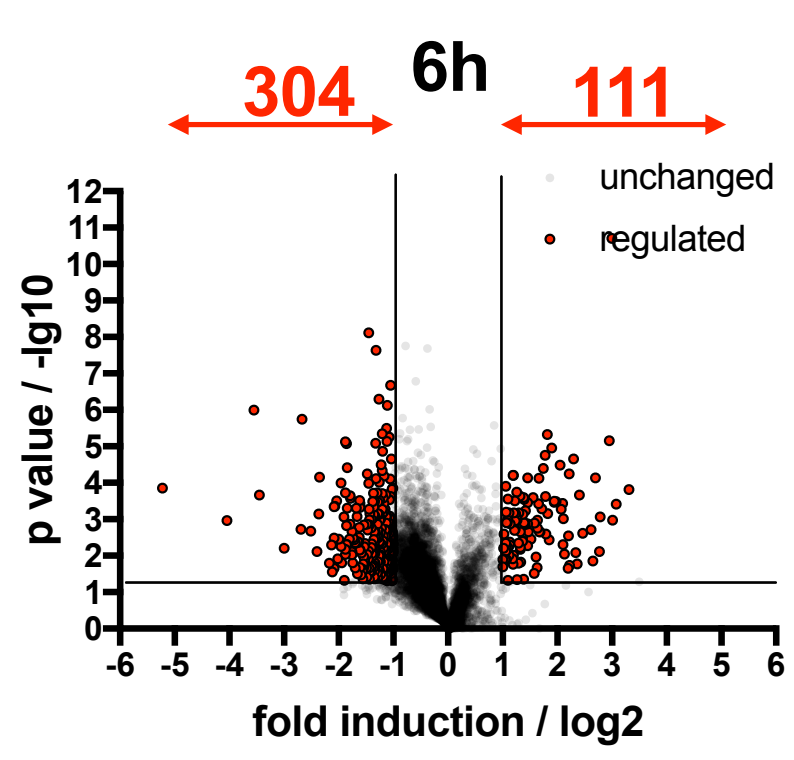

HyplL-6

$\underline{\text { IL-27 }}$

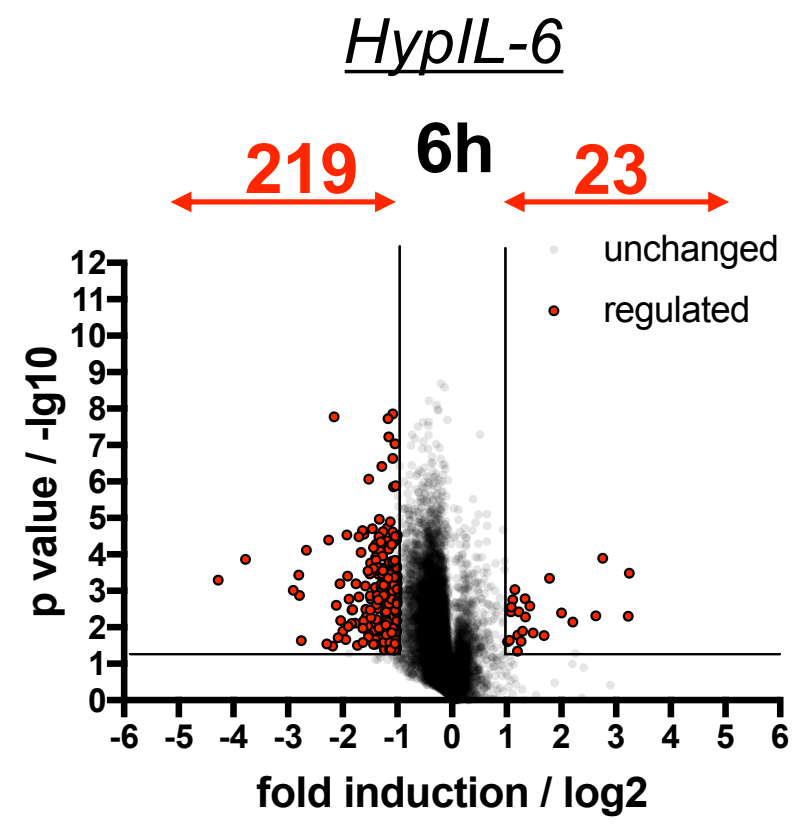

\section{HyplL-6 \\ (IL-27 regulated genes highlighted)}

c)

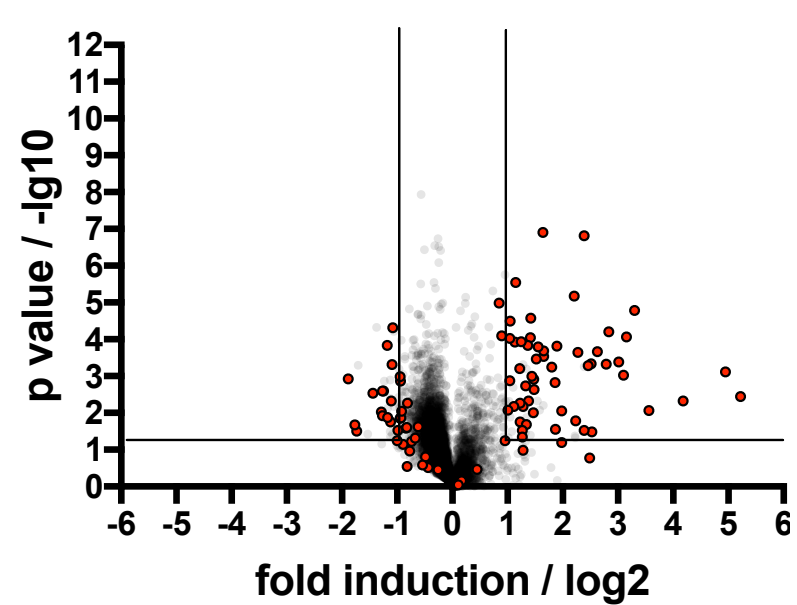

$6 h$

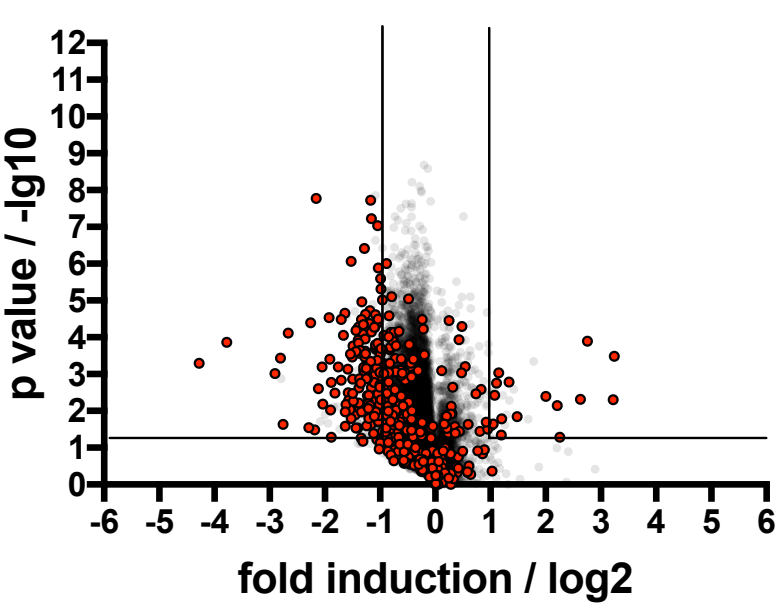

$24 h$

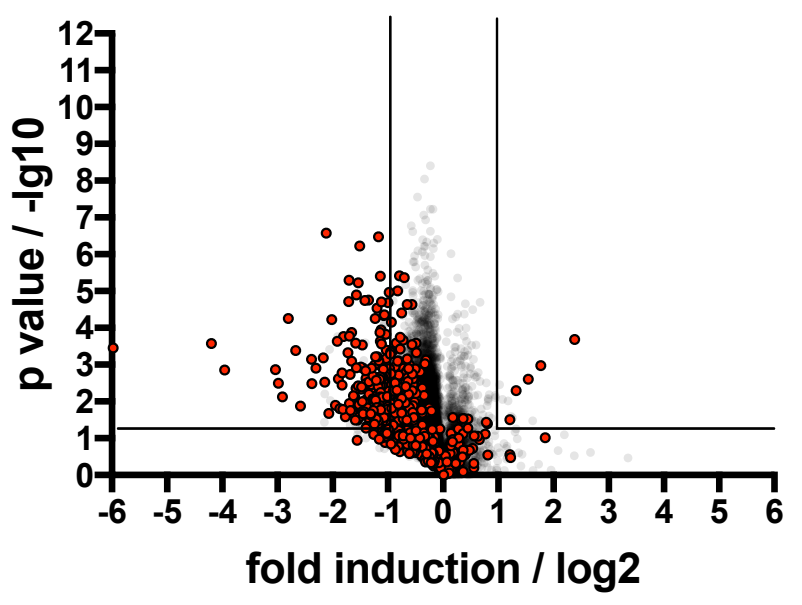




\section{supp. Fig. 10}

a)

Time $1 \mathrm{~h}$

$6 \mathrm{~h}$

$24 \mathrm{~h}$

Upregulated genes

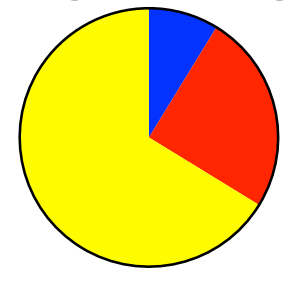

$\square$ IL-27

b)
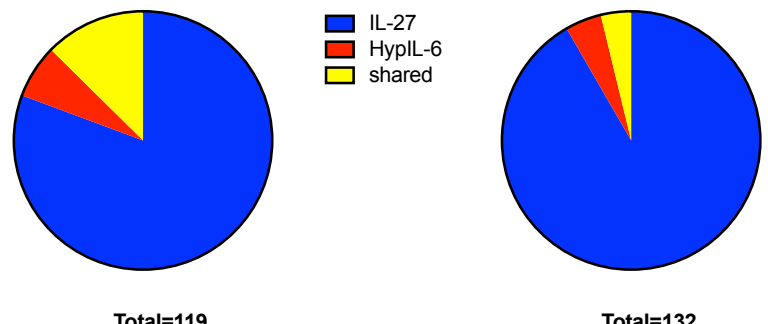

$\square$ IL-27

Total $=132$
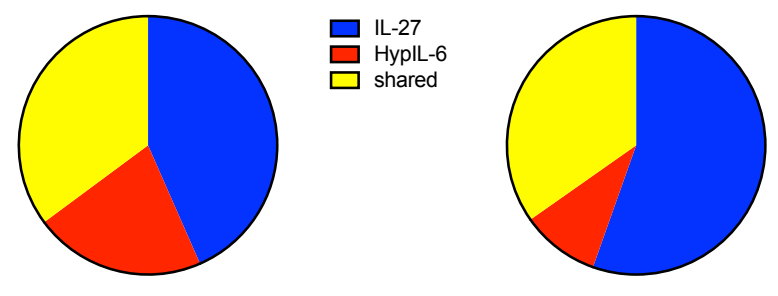

IL-27

$\square$ HyplL-6
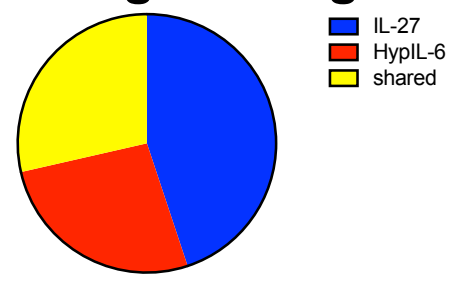

Total=387

Total $=590$

IL-27 top 30 up \& downregulated genes

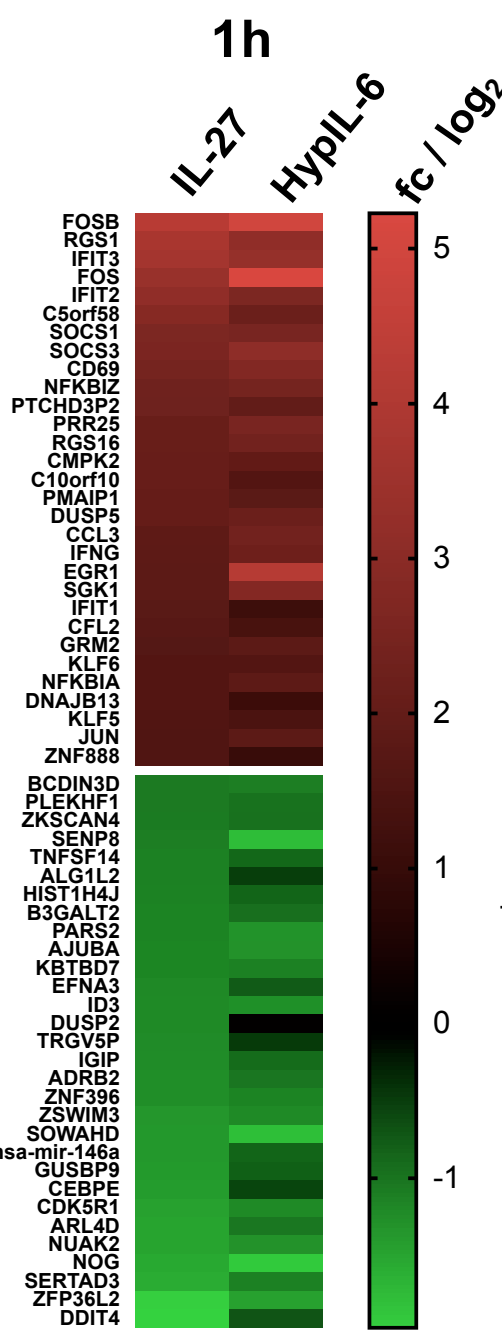

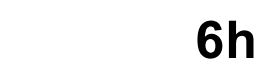

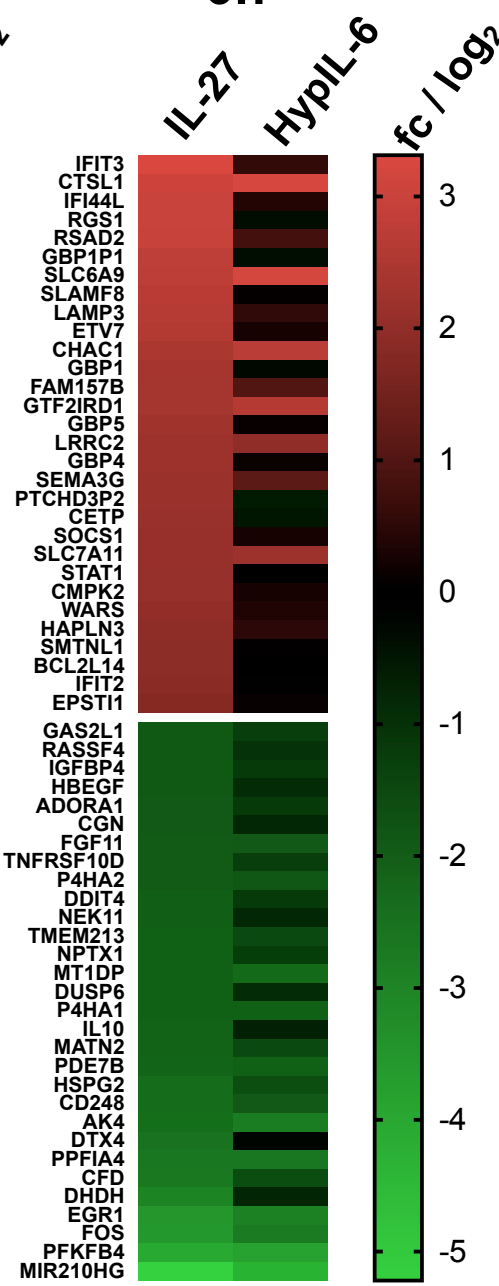

c)

Interferon Stimulated Genes (ISGs)

IL-27 HyplL-6

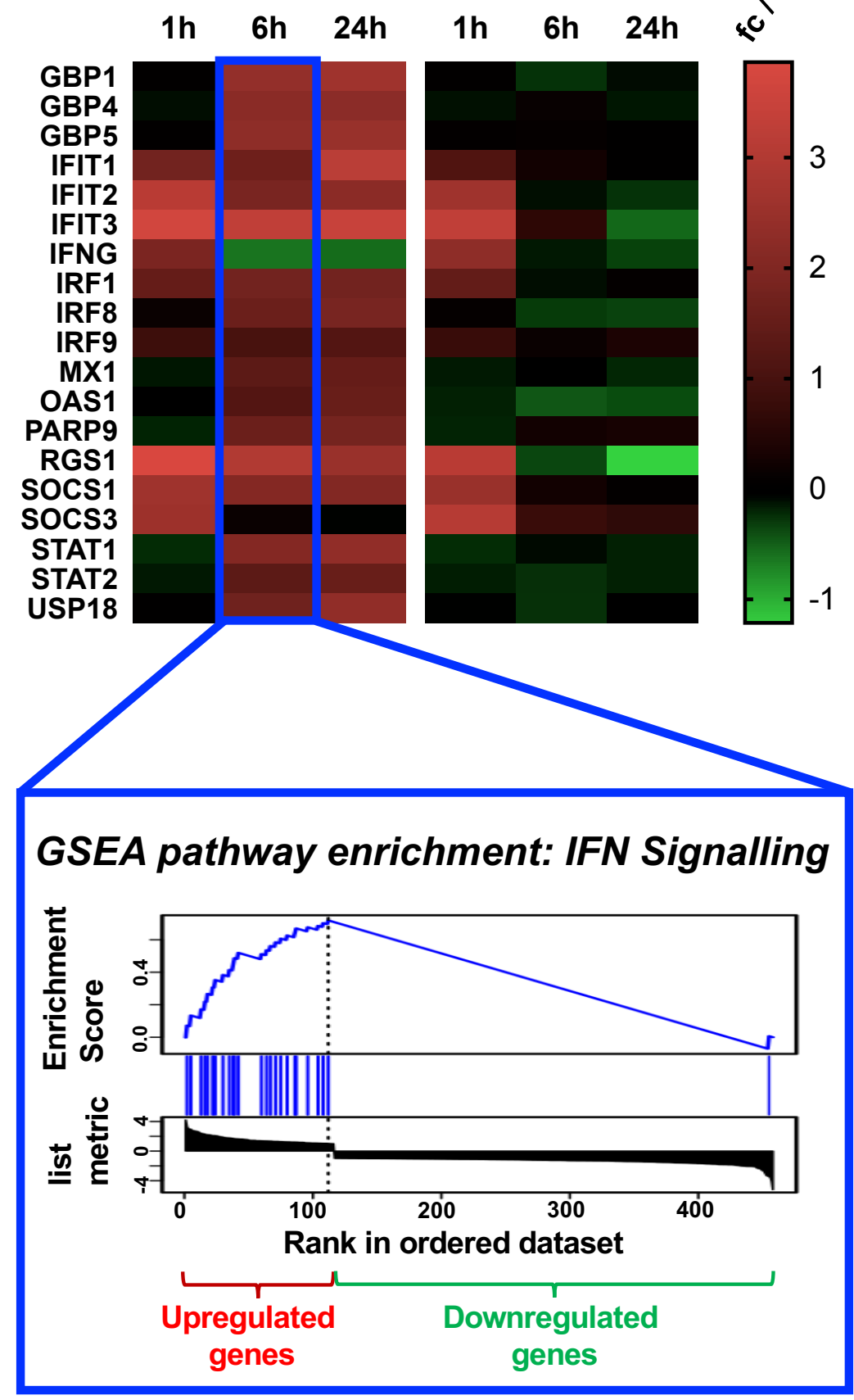




\section{supp. Fig. 11}

a)

Upregulated proteins

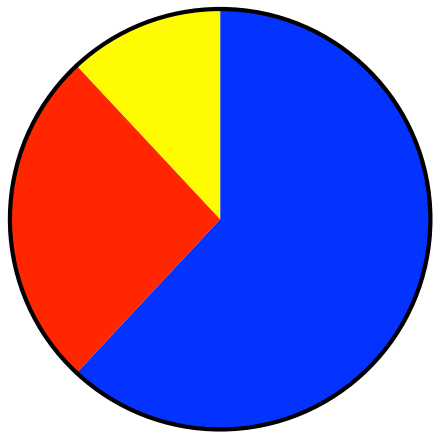

Total $=92$ $\square 1.96 \%$ IL-27

$\square 26.09 \%$ HyplL-6

$11.96 \%$ shared

b) GSEA pathway reactome: Interferon signalling

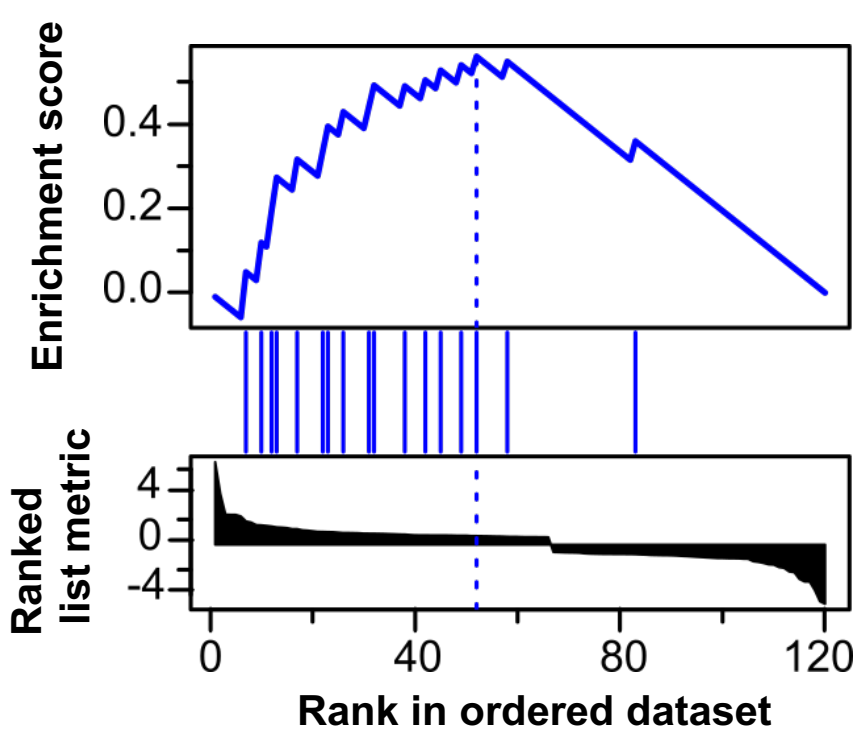

\section{IL-27 HypIL-6}

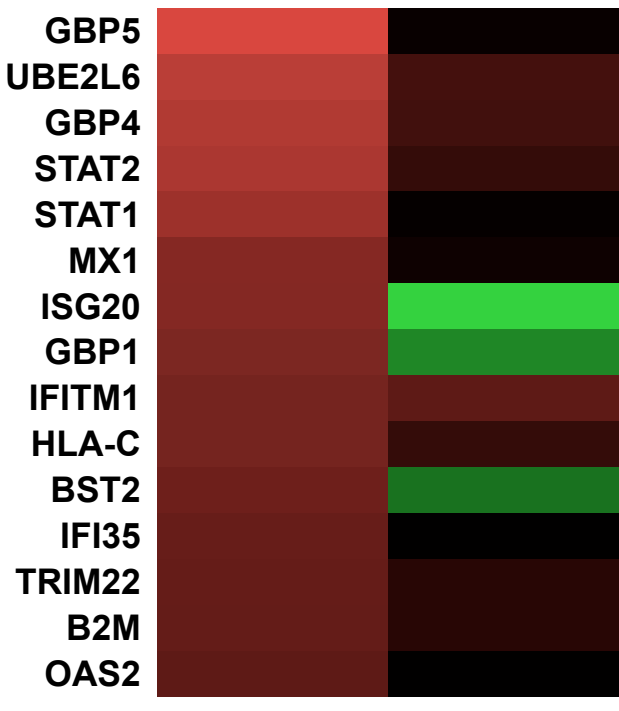

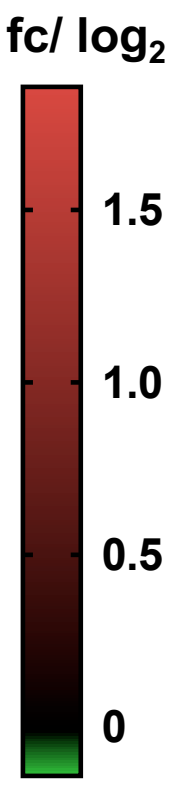

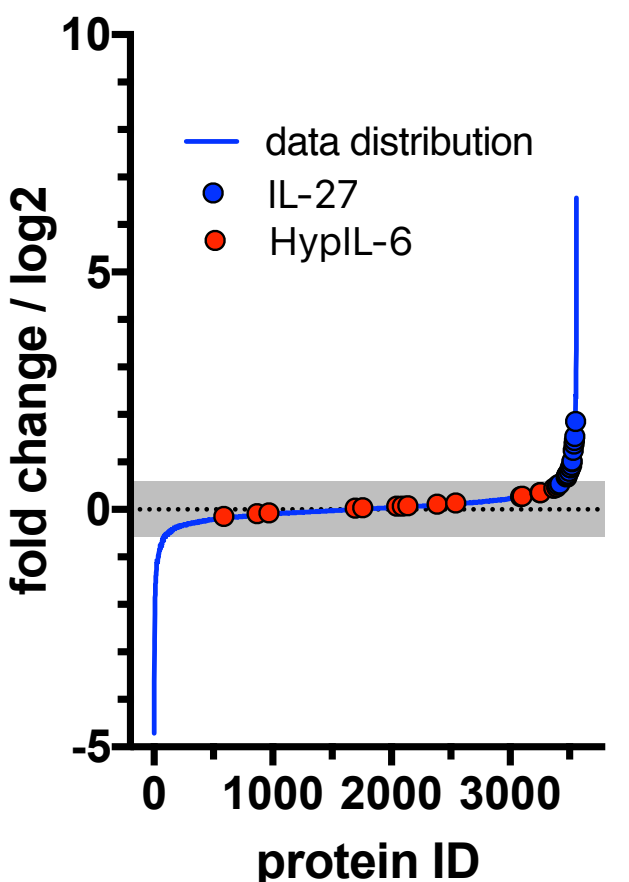

c) GSEA pathway reactome: Cytokine signalling and immune system

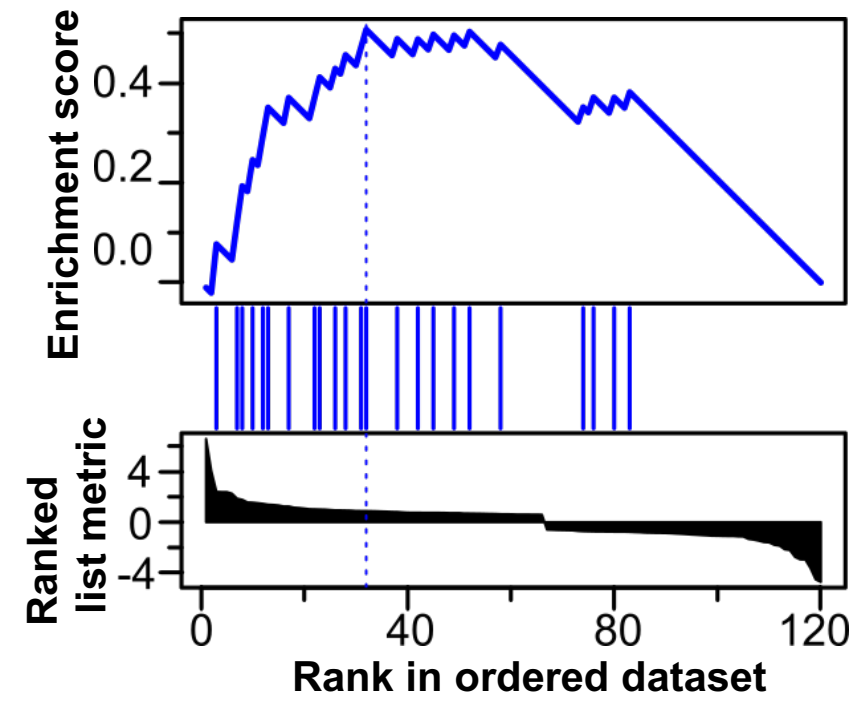

\section{IL-27 HyplL-6}

TGFB1
GBP5
RALA
UBE2L6
GBP4
STAT2
STAT1
MX1
ISG20
GBP1
MAPK14
IFITM1
HLA-C

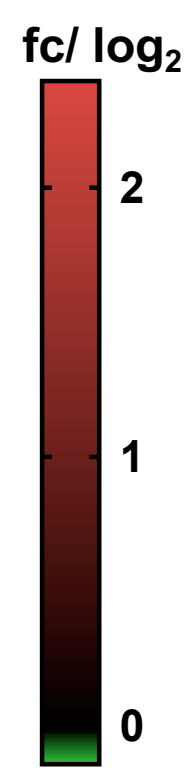

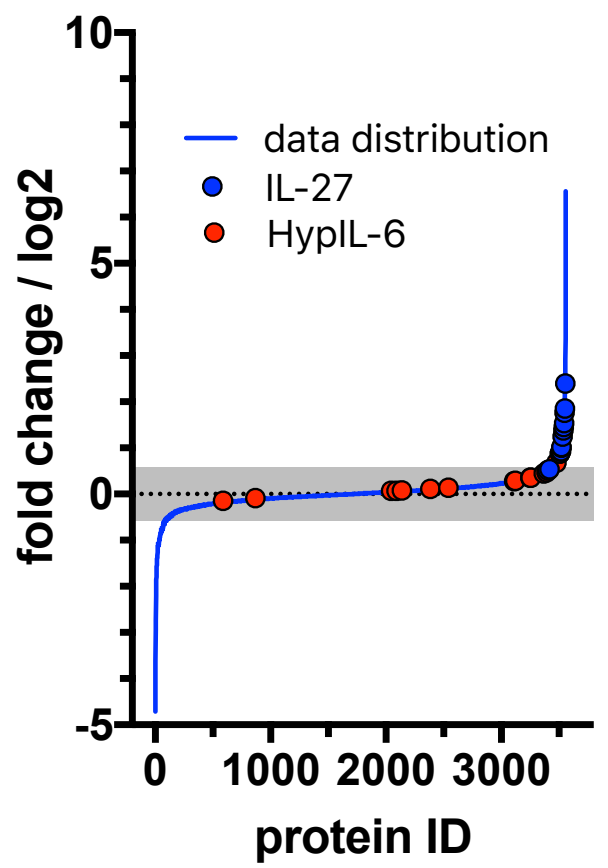




\section{supp. Fig. 12}

a)

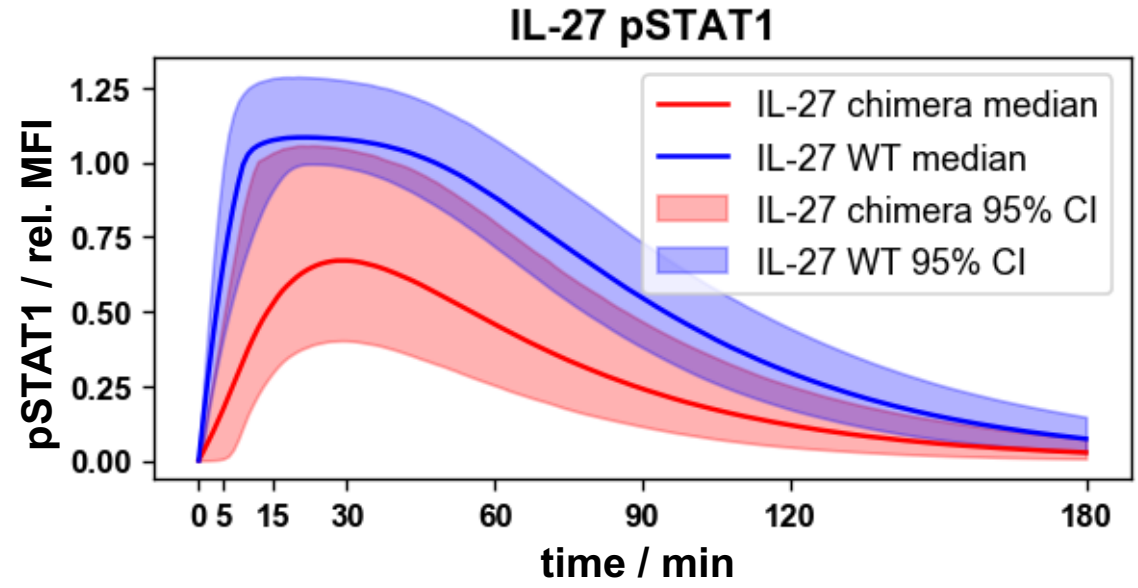

HypIL-6 pSTAT1

b)

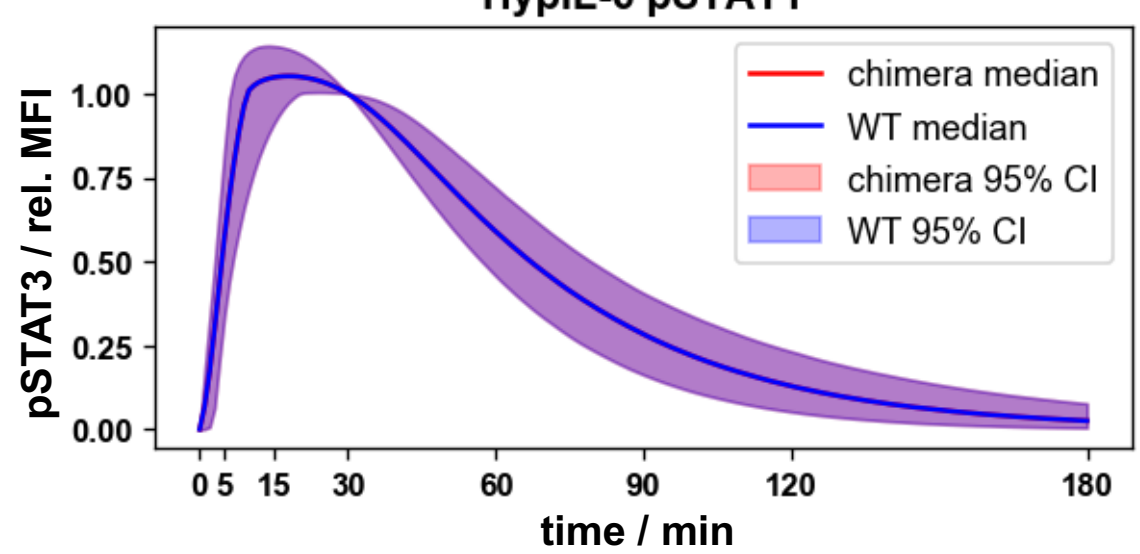

IL-27 pSTAT3

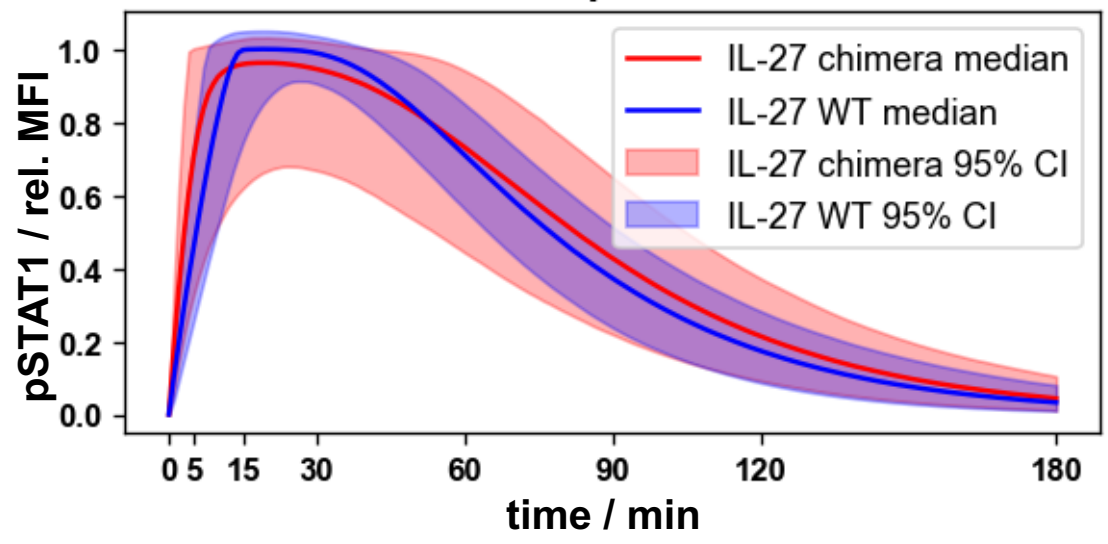

HypIL-6 pSTAT3

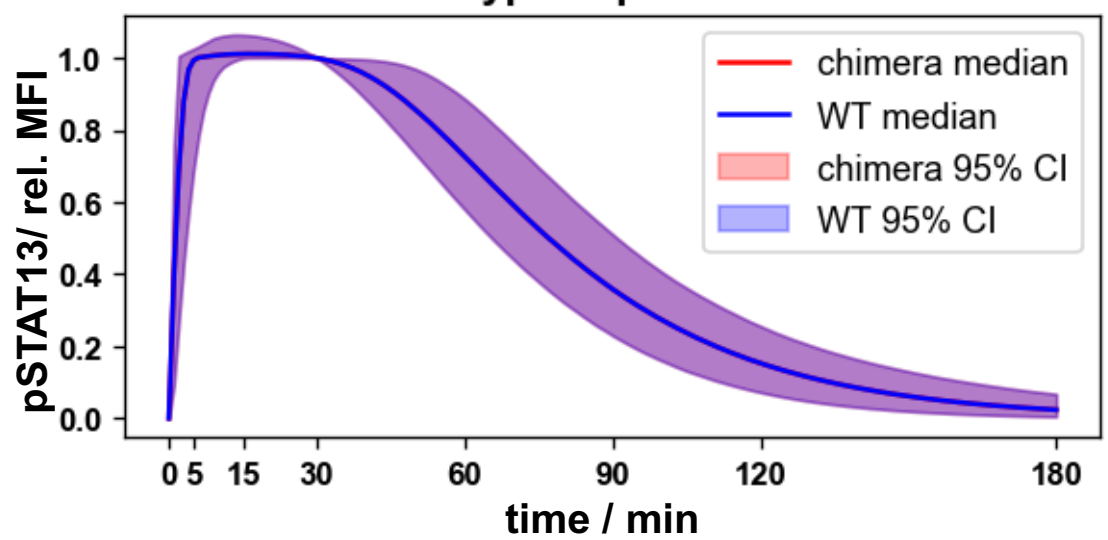

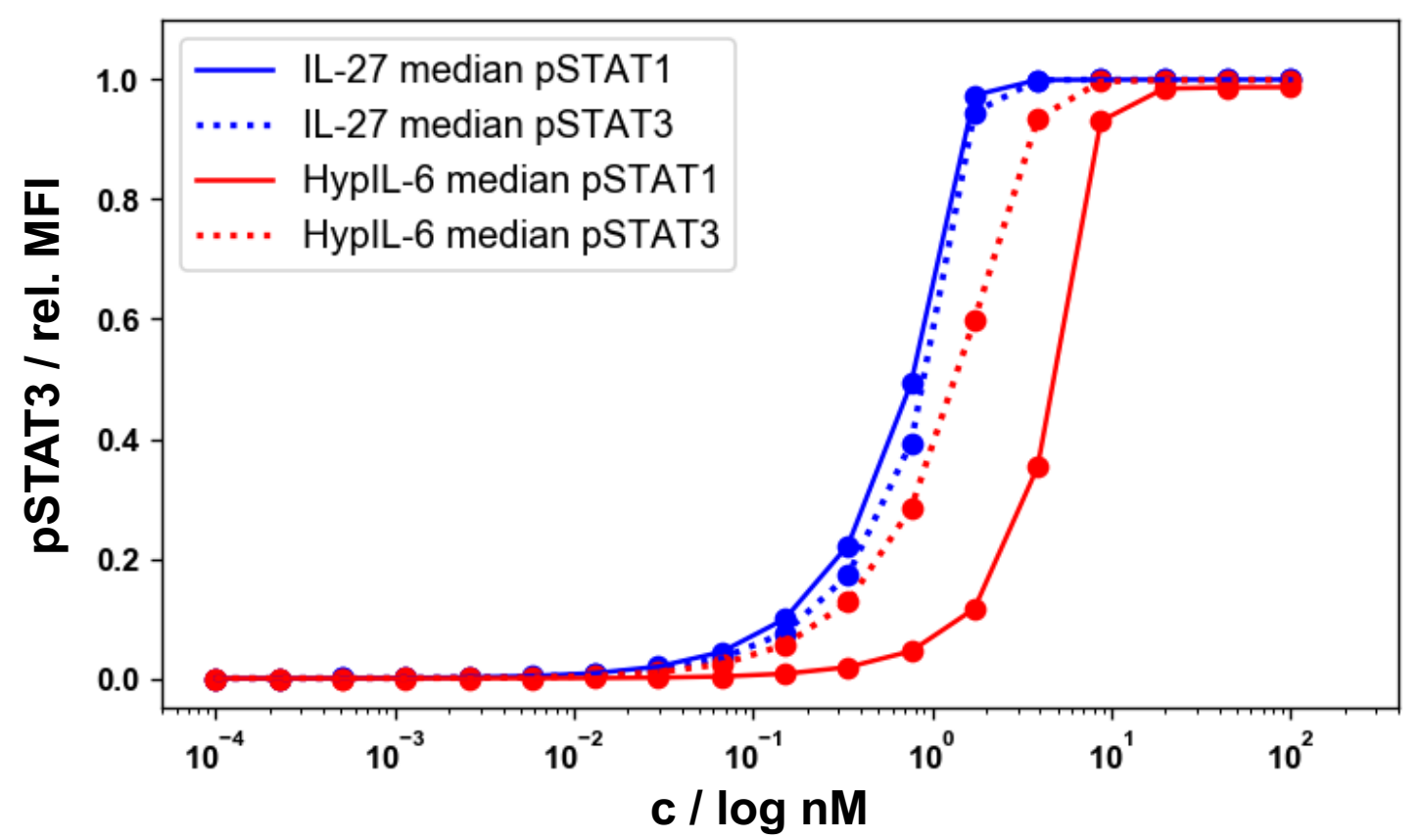

c)

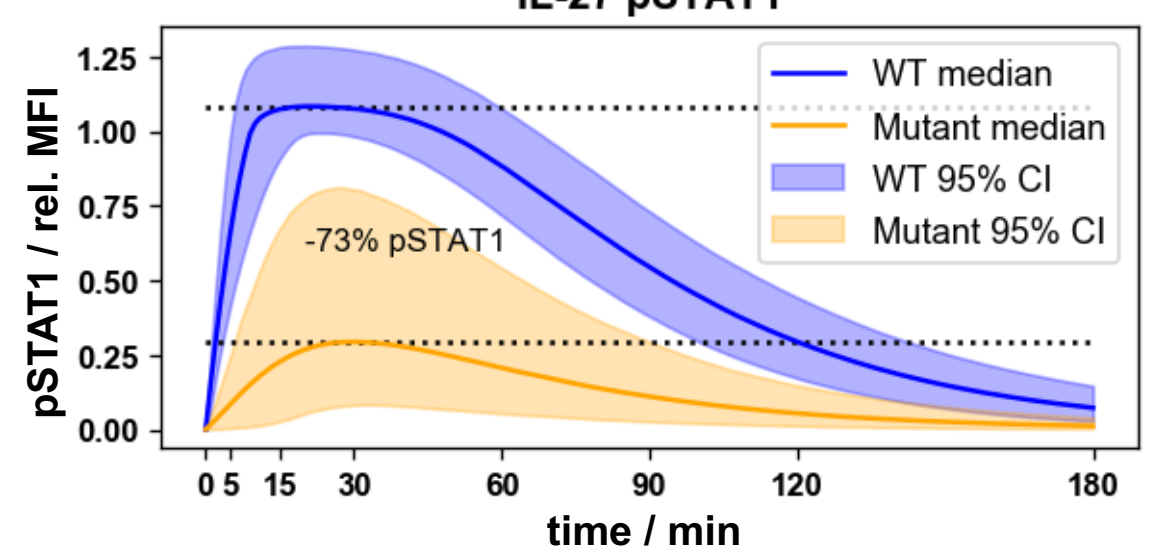

HypIL-6 pSTAT1

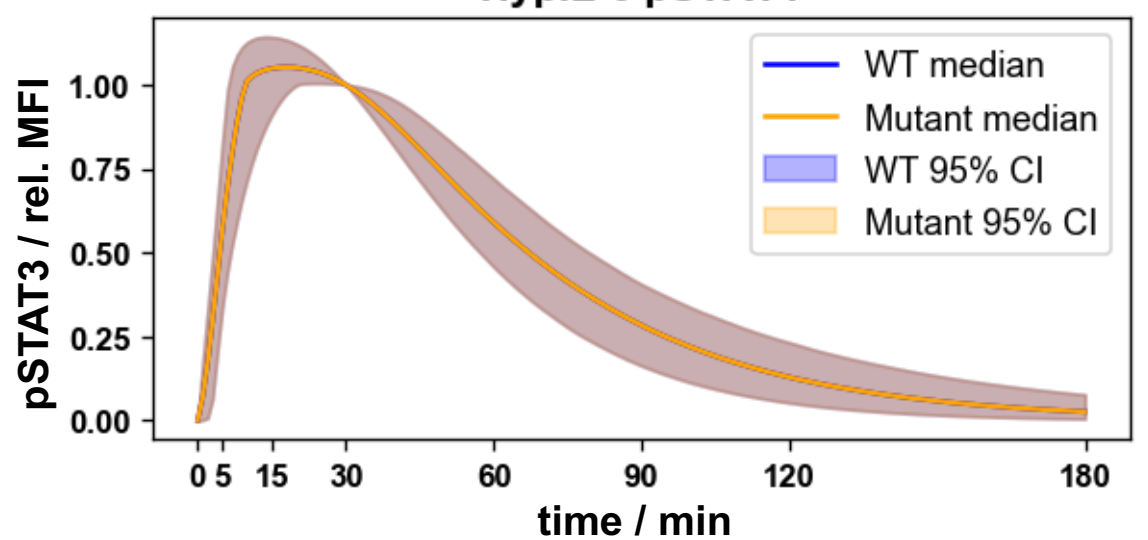

IL-27 pSTAT3

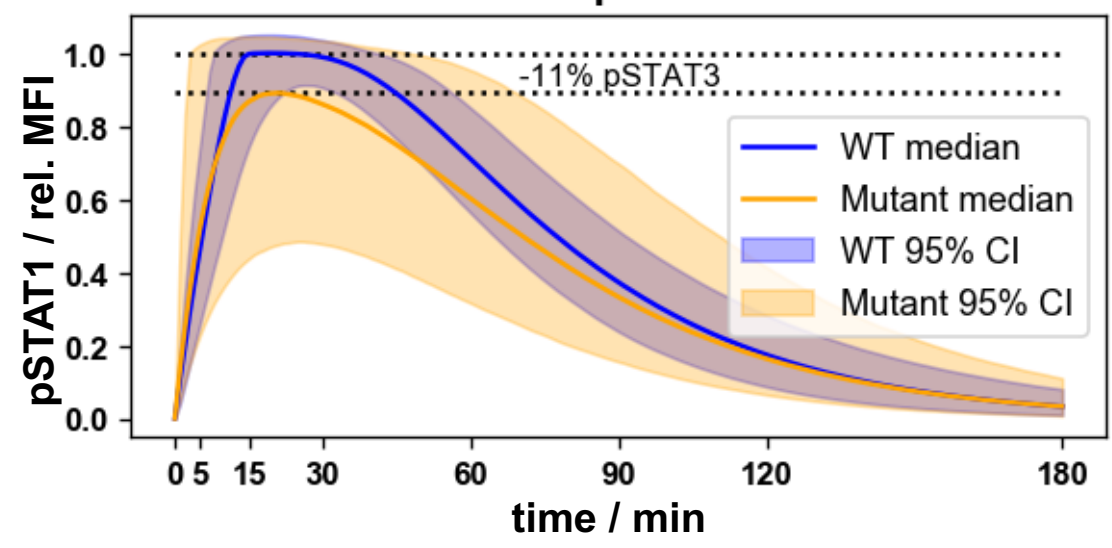

HypIL-6 pSTAT3

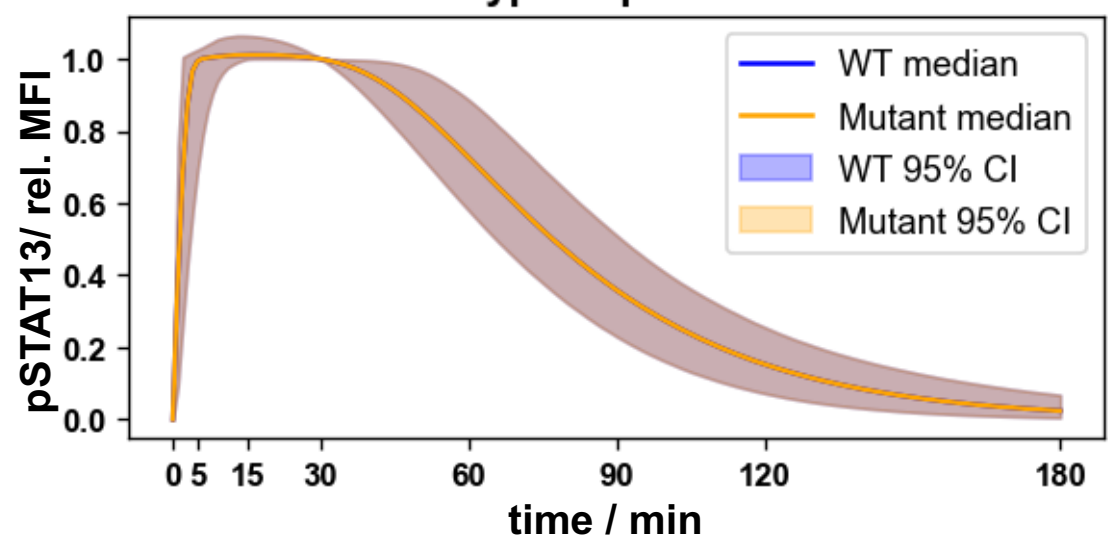



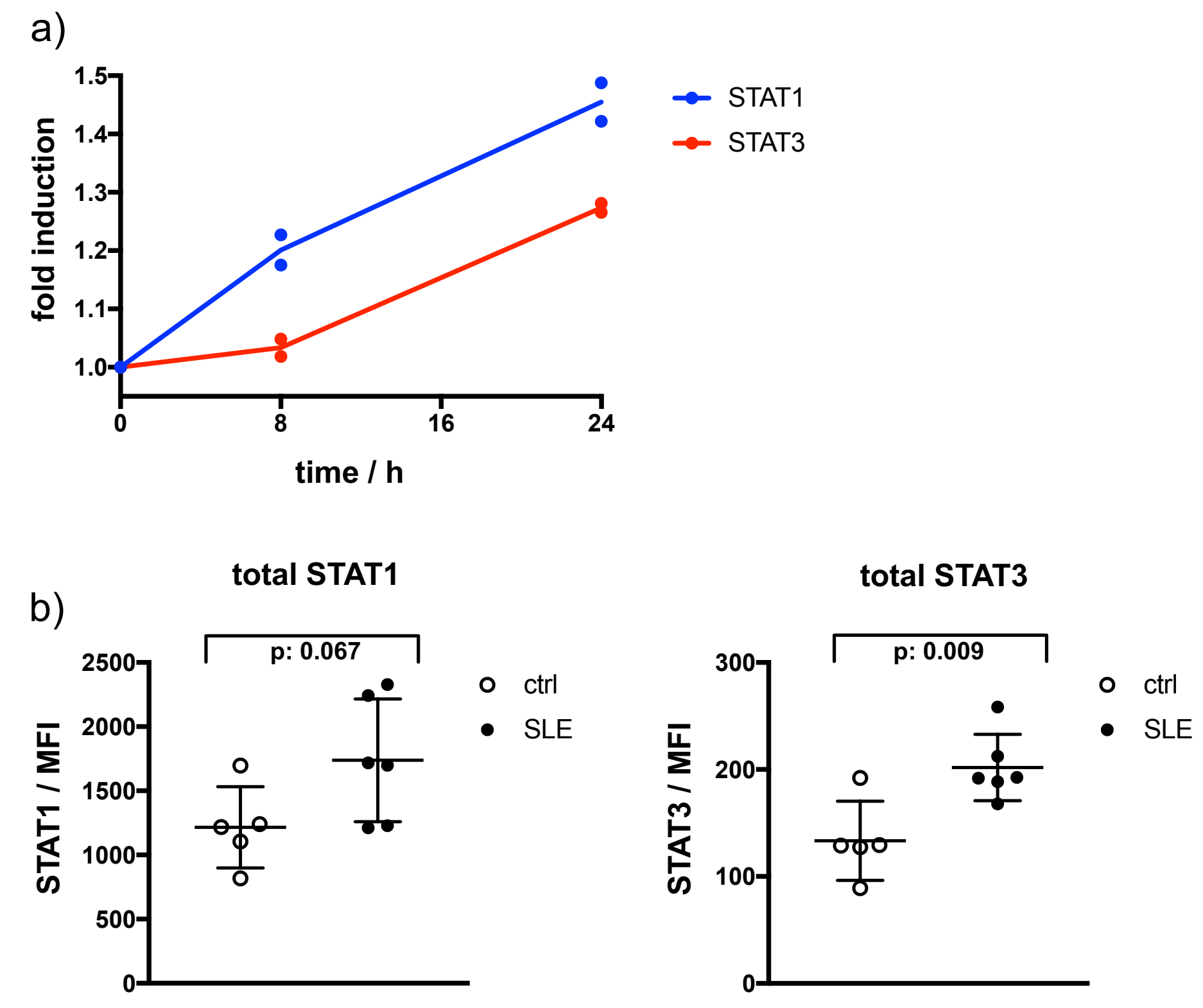

c)

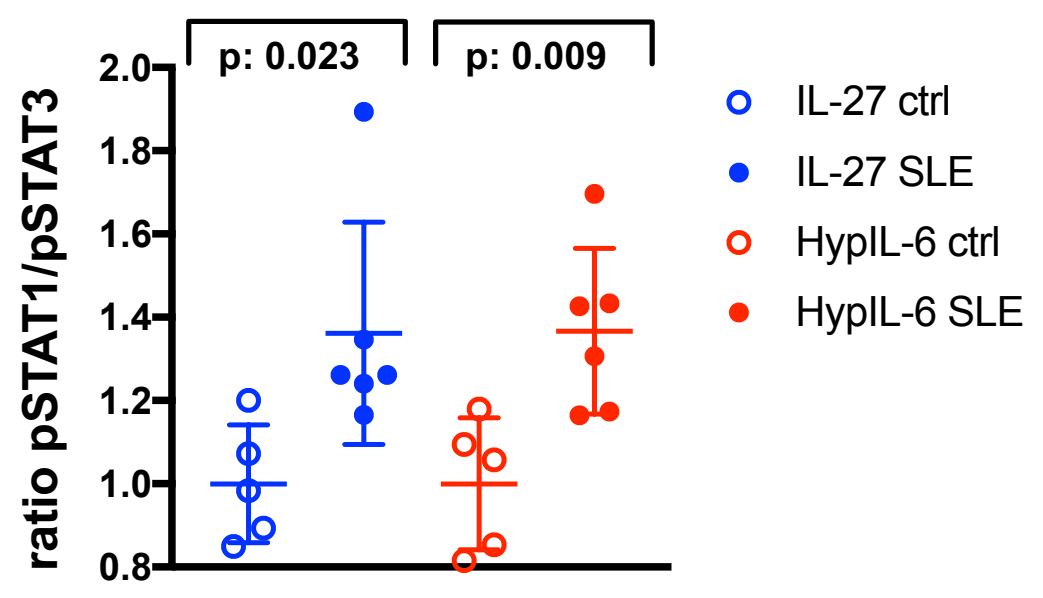

d) Tofacitinib titration - IL-27 signaling
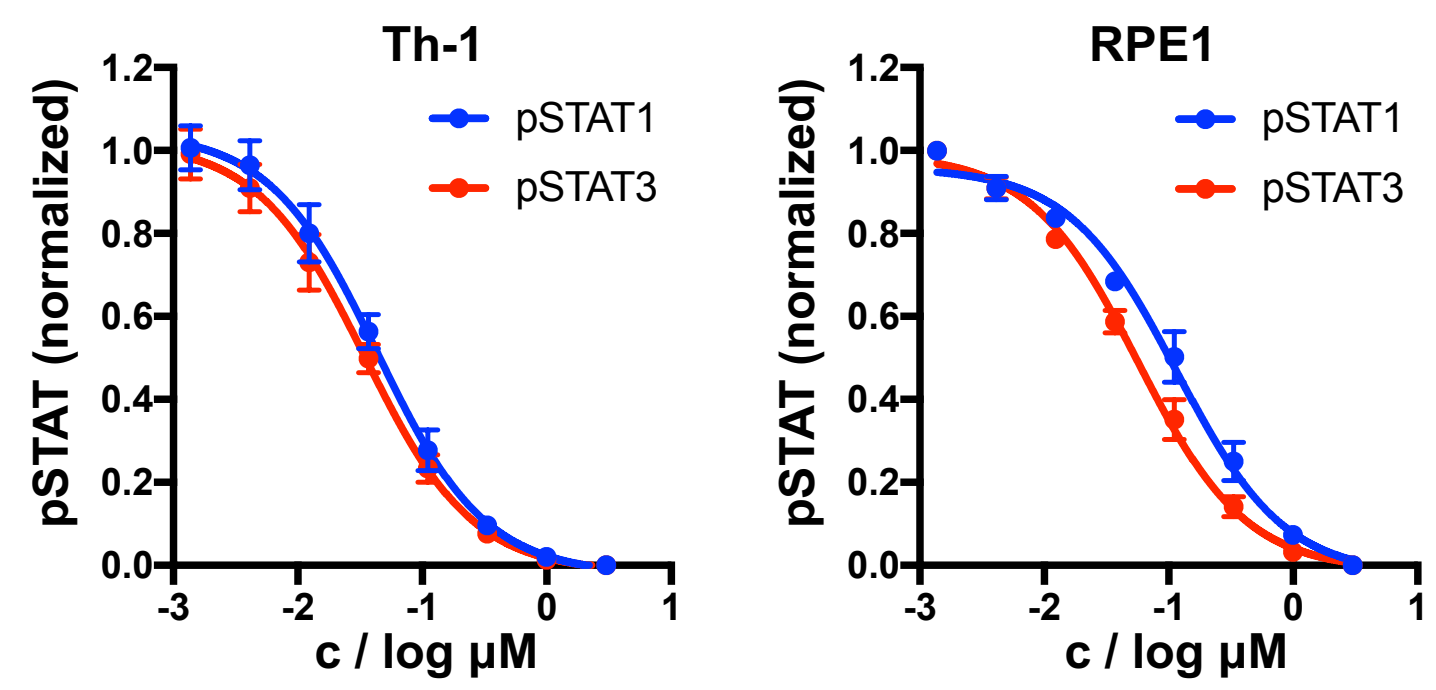